Genetic Diversity of Potato for Nitrogen Use Efficiency under Low Input Conditions in Ethiopia

Baye Berihun Getahun 


\section{Thesis committee}

\section{Promotor}

Prof. Dr Richard G.F. Visser

Professor of Plant Breeding

Wageningen University \& Research

\section{Co-promotor}

Dr C.G. van der Linden

Group leader, Plant Breeding

Wageningen University \& Research

\section{Other members}

Prof. Dr Paul C. Struik, Wageningen University \& Research

Prof. Dr Anton J. Haverkort, Wageningen University \& Research and Nigde University, Turkey

Dr Frits K. van Evert, Wageningen University \& Research

Dr Peter G. Vos, HZPC Holland BV, Metselawier

This research was conducted under the auspices of the Graduate School of Experimental Plant Sciences. 


\title{
Genetic Diversity of Potato for Nitrogen Use Efficiency under Low Input Conditions in Ethiopia
}

\author{
Baye Berihun Getahun
}

\section{Thesis}

submitted in fulfillment of the requirements for the degree of doctor at Wageningen University

by the authority of the Rector Magnificus,

Prof. Dr A.P.J. Mol,

in the presence of the

Thesis Committee appointed by the Academic Board

to be defended in public

on Monday 18 September 2017

at 1.30 p.m. in the Aula. 
Baye Berihun Getahun

Genetic Diversity of Potato for Nitrogen Use Efficiency under Low Input Conditions in Ethiopia,

219 pages.

PhD thesis, Wageningen University, Wageningen, the Netherlands (2017)

With references, with summaries in Dutch and English

ISBN: 978-94-6343-659-5

DOI: https://doi.org/10.18174/420903 


\section{Contents}

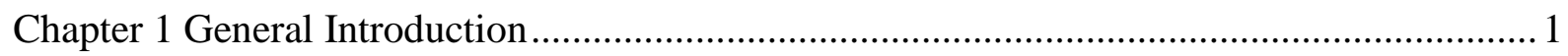

Chapter 2 Genetic diversity of potato cultivars for nitrogen use efficiency under contrasting

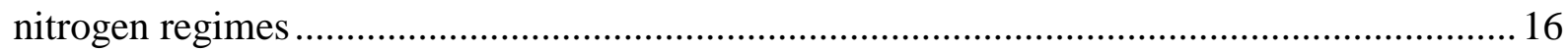

Chapter 3 Identification of QTLs associated with nitrogen use efficiency and related traits in a diploid backcross potato population

Chapter 4 Genome-wide association mapping of nitrogen use efficiency and related traits in potato under contrasting nitrogen regimes.

Chpater 5 Genotype-by-Environment interaction for nitrogen use efficiency of potato (Solanum tuberosum L.) under different growing conditions in North western Ethiopia. ...... 95

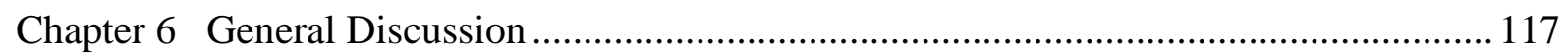

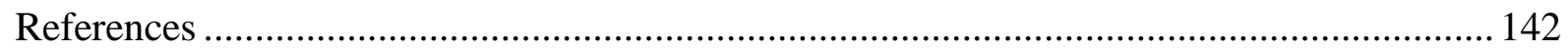

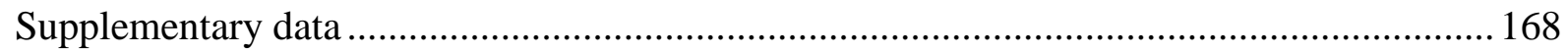

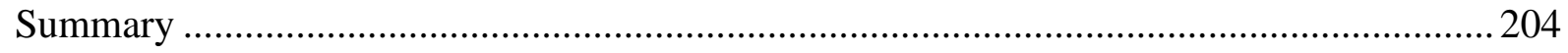

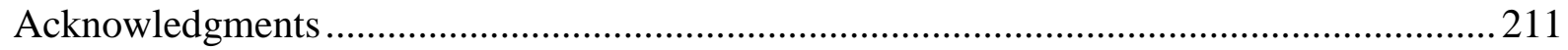

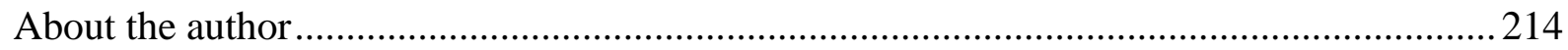

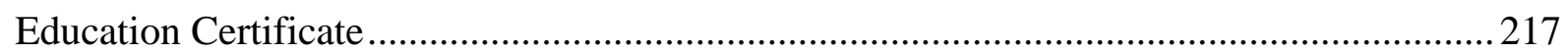


Chapter 1

General Introduction 


\section{The potato: Origin, expansion and production trends}

Potato (Solanum tuberosum $L$ ) is the $3^{\text {rd }}$ most important food crop in the world after wheat and rice in terms of human consumption. The crop originated in the Andes Mountains of South America, where it has served as a staple in the diet of native people for a long time. It was domesticated 7,000 years ago near Lake Titicaca, (on the border between Bolivia and Peru), where the greatest diversity of wild species is currently found (Simmonds, 1995; Spooner et al., 2005). The successful journey of potato around the globe started in the $16^{\text {th }}$ century, when the Spanish brought it to Europe from the South American Andes. From Europe, the crop found its way to Asia and Africa in the $17^{\text {th }}$ and in the $19^{\text {th }}$ century, respectively (Hawkes, 1994).

In modern agriculture the crop is by far the most widely cultivated tuber-bearing species, with a production of 365 million tonnes fresh weight of tubers produced in 2012 from 21 million hectares of land (FAOSTAT, 2012). Globally, more than a billion people utilize potato, making it a critical crop in terms of food security in the face of population growth and increased hunger. In many developing countries the poorest and most undernourished farm households depend on potatoes as a primary and secondary food and nutrition source (FAOSTAT, 2008). The overall trend in potato production from 1991 to 2012 shows a 27\% increase, from 268 million tons (Mt) to 365 million tons (FAOSTAT, 2012). Globally, in developing countries, the production of potato has increased from 28 million metric tons in 1963 to 149 million metric tons in 2005, which more than offsets the drop in production in industrialized countries (FAOSTAT, 2011).

Several governments are appreciating the economic and nutritional importance of potato and are developing appropriate policies to encourage its production and consumption with medium and long-term strategies for sustainable potato development. According to the International Potato Center CIP, as much as 50\% of the increased food production that will be necessary to produce to meet the demands in the next 20 years will come from potatoes in China, the world's biggest producer of potatoes (2012). Generally remarkable progress has been made in potato production and productivity levels in certain countries of the world, whereas in other countries the progress is limited. The opportunities for further development of the potato industry appear to be considerable, however at the same time the production constraints to be addressed are huge. 


\section{Production constraints of potato}

In the past, potato breeding programs focused primarily on developing varieties with maximum yield and quality, good storage properties, low incidence of physiological disorders and improved genetic resistance to major pests and pathogens that adversely affect the potato industry. Production constraints related to nutrient use efficiency were not considered as a priority research agenda for potato. Nowadays, problems related to nitrogen fertilization and use have become more critical, economically as well as environmentally. According to Hirel et al. (2011), nitrogen availability has become the second priority production constraint after drought to be addressed in the crop's abiotic stress improvement program. A dramatic increase has occurred in crop yield through global utilization of synthetic nitrogen $(\mathrm{N})$, increasing from 11.6 million tons in 1961 to 104 million tons in 2006 (Hoang et al., 2010). Consequently, high crop productivity is heavily dependent on nitrogen fertilization. However, the increase in crop yield and synthetic $\mathrm{N}$ consumption has resulted in increased air pollution, alteration of the quality of surface and ground water resources through runoff and leaching, increased greenhouse gas emission and coastal eutrophication (Billen et al., 2013). Moreover, $\mathrm{N}$ fertilizer prices are currently very high, which is a direct result of the cumulative world demand for the limited fossil fuel reserves. The high costs and low returns of fertilizer use particularly burdens smallholder farmers in developing countries, forcing them to grow their crops under suboptimal N supply (Lafitte and Edmeades, 1994; Dethier et al., 2012).

Potato is one of the major contributors to the leaching of nitrogen into the groundwater. Different research results indicate that adequate nitrogen application in the growing season is required to realize high potato yield and quality (Stark et al., 2004). However, effective management of nitrogenous fertilizers is a challenging task in potato production. Elevated ground water nitrate concentrations have been attributed to commercial potato production (Hill 1986; Richards et al., 1990) and significant emissions of nitrous oxide (a greenhouse gas) have been measured from potato fields (Flessa et al., 2002; Burton et al., 2008). Moreover, because of the high cost of $\mathrm{N}$ fertilizer, potato is one of the crops that suffer from being cultivated at $\mathrm{N}$ limiting conditions in tropical and sub-tropical regions of developing countries like Ethiopia.

Potato was introduced in Ethiopia in 1858 by the German Botanist Schimper (Pankhurst 1964), and used to be an important garden crop in many parts of the country. Currently it is one of the major food crops produced in large farm lands, especially in the highland parts of the country. The production area has increased more than fivefold from 30,000 ha from the 
1970s (Kidane Mariam, 1979) to more than 179,000 ha today (CSA, 2014). Potato can potentially be cultivated on $70 \%$ of the 10 million hectares of arable land in the country (FAOSTAT, 2008). However, the average productivity in Ethiopia is below 10 tons/ha, which is far below the country's potential. Poor soil fertility is one of the major production constraints that contribute to this low productivity. The Ethiopian potato breeding program has developed a number of improved potato varieties under high input conditions, while most farmers are producing in low input farming systems. According to Mulat (1999), the amount of fertilizer applied by most Ethiopian farmers to crops is below the recommended level; for instance, only $35 \%$ of the total cereal production area receives chemical fertilizer, and the average fertilizer use of Ethiopia is about $17 \mathrm{~kg} / \mathrm{ha}$, which is very low by any standard (Agriculture For Impact, 2014).

As the need for food production increases with an increasing population growth, it is important that strategies are developed to enhance the nutrient uptake and utilization efficiencies under both conditions of sufficient fertilization and conditions with chronic shortage of nitrogen. Several different strategies are currently being pursued to address problems associated with inefficient agricultural systems and the Nitrogen $(\mathrm{N})$ cascade (Galloway et al., 2002). One of the strategies is breeding aimed at developing crop varieties that are more efficient at capturing soil nitrogen $(\mathrm{N})$, thereby decreasing $\mathrm{N}$ leaching and denitrification losses and reducing plant $\mathrm{N}$ requirements (Cassman et al., 2002). To develop crop varieties that are more $\mathrm{N}$ efficient, knowledge of the genetic diversity and relationships among the genotypes is very useful in order to recognize gene pools that can be utilized for growth improvement under $\mathrm{N}$-limiting conditions and more efficient use of nitrogen, to identify the gaps in germplasm collections and to realize effective conservation and utilization strategies (Esfahani et al., 2009).

\section{Genetic diversity of potato}

Broadening the genetic basis of a gene pool that is used as a gene source is highly desirable for any crop improvement program, because genetic diversity provides buffering against losses due to abiotic and biotic stresses. Cultivated potato and its wild relatives group under the genus solanum, which is the largest genus in the family Solanaceae with 2000 species (Volkov et al., 2003). All tuber-bearing Solanum species are grouped in the section Petota, and this section is subdivided into two subsections, Potatoe and Estolonifera (Hawkes, 1990). The subsection Potatoe comprises all tuber bearing potatoes, while the two non-tuber bearing series Etuberosa and Juglandifolia are grouped in subsection Estolonifera. Subsection 
Potatoe (the tuber-bearing species) members have unique reproductive characteristics, that include the possibility to use both vegetative and sexual reproduction, presence of different ploidy levels, existence of an endosperm dosage system which controls interspecific hybridization, production of gametes with unreduced chromosome number and introgression (sexual compatibility among species) (Carputo and Barone, 2005). These unique characteristics may be of paramount importance in genetic diversity, breeding and evolution studies, however they increase the complexity of the taxonomic classification in section Petota, as it is difficult to differentiate one species from the other (Spooner and Bamberg, 1994; Spooner, 2009). Cultivated potato species have a haploid chromosome number of 12 with ploidy level ranging from diploid $(2 n=2 x=24)$ to hexaploid $(2 n=6 x=72)$. The majority of the diploid species are self-incompatible, while the tetraploid and hexaploids are selfcompatible (Hawkes, 1990).

Potato has an extremely diverse gene pool that can be utilized in different potato breeding programs (see Fig. 1 for example in tuber genetic diversity) (Watanabe, 2002). However, due to loss of genotypes in the journey from South America to Europe, the genetic basis for commercial potato cultivars is narrow, and only a few stocks of the cultivated potato from South America were introduced in Europe (Ross 1986). After introduction, the genetic diversity was reduced further in Europe by the selection of short day phenotypes, and still further limited by the blight epidemics in the mid-nineteenth century. Moreover, exposure to high $\mathrm{N}$ fertilizer application rates in potato breeding and variety development resulted in cultivars that are more responsive to high $\mathrm{N}$ but less capable of producing optimum yields under minimum or insufficient nitrogen supply (Rowe, 1969; Hawkes, 1990). However, not all cultivars that are more responsive to high $\mathrm{N}$ are necessarily inefficient in using $\mathrm{N}$. Cultivars that have good genetic potential for Nitrogen use efficiency and $\mathrm{N}$ responsiveness may be available in commercial cultivars. The ideal genotypes have both high genetic NUE and high $\mathrm{N}$ responsiveness, provided that the traits for NUE and $\mathrm{N}$ responsiveness are not genetically linked (weak linkage) (Han et al., 2015). Thus, in order to improve $\mathrm{N}$ use efficiency both in high $\mathrm{N}$ input and low $\mathrm{N}$ input systems, the available genetic resources that are found in the hands of the subsistence potato growers including commercial potato cultivars, traditional cultivars and the wild species will need to be exploited. 


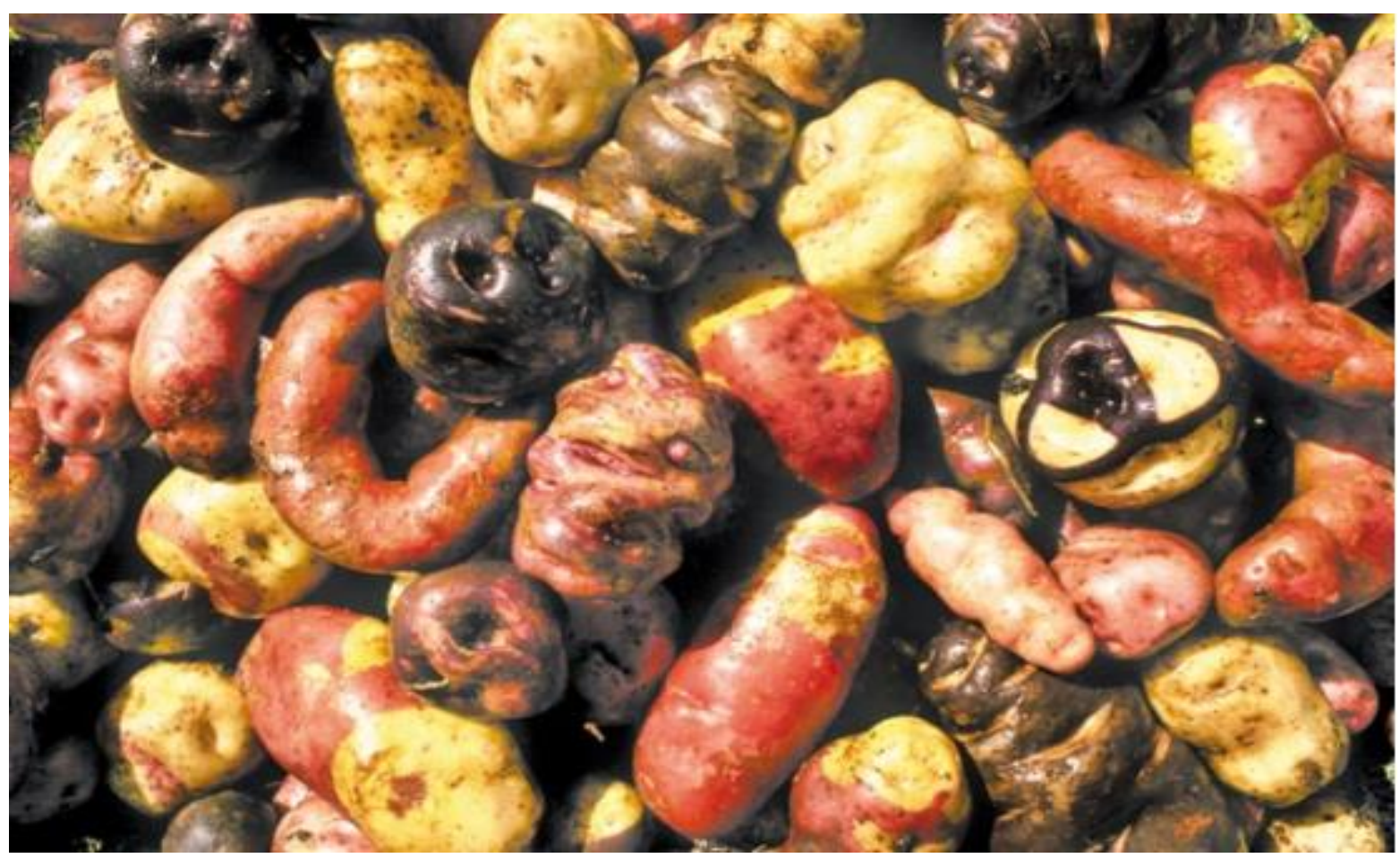

Fig. 1: Phenotypic variation of tuber shape and colour caused by genetic diversity of potato.

Source: http://cipotato.org/potato/facts

\section{Nitrogen use efficiency: Definitions and concepts}

Nitrogen use efficiency (NUE) was defined by different authors in different ways, depending on the objective of the study and the crop under study. For example, Moll et al. (1982) defined NUE as the yield per unit of nitrogen resource available to the plant. Bock (1984) defined NUE as the total dry weight of the plant per unit of soil N. In this thesis we follow the definition of Moll et al. (1982). NUE is a function of two primary components; N uptake efficiency (NUpE) and $\mathrm{N}$ utilization efficiency (NUtE). $\mathrm{N}$ uptake efficiency (NUpE) is defined as the whole plant $\mathrm{N}$ content per unit of $\mathrm{N}$ supplied. It is the ability of the crop to take up nitrogen from the soil and store in the plant. The NUpE of genotypes depends on the plant root architecture and functioning in the soil. $\mathrm{N}$ utilization efficiency (NUtE) is the ratio of plant dry matter content per unit of $\mathrm{N}$ taken up by the plant. It is the efficiency of the genotype to fix carbon for the nitrogen taken up from the soil, which includes the process of photosynthesis, canopy development and longevity, nitrogen remobilization from all tissues to grain (sink) during grain filling in cereals, or bulking in root and tuber crops (Good et al., 2004). These two components have been compared between fertilization levels and crop varieties in order to decide which component is more important for the overall NUE, however the results are often inconsistent and depending on the fertilization level and the crop species. For example, in maize the relative contribution of NUpE for the overall NUE was more at high $\mathrm{N}$, while at low N, NUtE contributed more to the total NUE variation (Moll et al., 1982; 
Bertin and Gallis, 2000). This result seems to be opposite to wheat, for which NUpE contributed more to NUE at low N fertilization levels (Le Gouis et al., 2000). Overall these findings suggest that the contribution of NUpE and NUtE to the total NUE variation depends on the level of applied $\mathrm{N}$ fertilizer and the crop species or genotypes under evaluation.

\section{Potato $\mathrm{N}$ requirement}

Crop nitrogen uptake under non-limiting $\mathrm{N}$ supply is primarily determined by crop growth (Gastal and Lemaire, 2002) and there is generally a close relationship between plant $\mathrm{N}$ uptake and plant dry matter accumulation (Vos, 1997). In potato, before emergence, growth is primarily controlled by soil temperature (Yuan and Bland, 2005) and the seed tuber physiological maturity (Allen and Scott, 1992); it is hardly affected by soil N. After emergence potatoes require a steady supply of nutrients (Stark et al., 2004; Westermann, 2005). Deficiencies or variations of soluble nutrients (especially N) cause poor vine health, reduced pathogen and insect resistance, resulting in decreased tuber yields and tuber quality (Ojala et al., 1990, Stark et al., 2004).

Increased fertilizer $\mathrm{N}$ application increases leaf area index (LAI) through increased size and number of leaves (Vos 1995). It can also increase leaf longevity and rate of photosynthesis (Vos and Biemond 1992), thereby increasing length of maturity period. $\mathrm{N}$ availability has also an effect on onset of tuberization (Ewing and Struik, 1992), final tuber yield and harvest index of potato (Vos \& MacKerron, 2000). The amount of $\mathrm{N}$ fertilizer applied has also its own influence on the nitrogen uptake and utilization efficiency of potato. $\mathrm{N}$ use and utilization efficiency decreased with increasing fertilizer $\mathrm{N}$ rate (Zebarth et al., 2004a, Ospina et al., 2014). The crop's ability to take up available soil nitrogen is typically low; $50 \%$ lower than other crops (Tyler et al., 1983; Dilz 1987). This is at least partly attributed to its shallow rooting system and therefore inefficiency N uptake (Yamaguchi et al., 1990; Peralta et al., 2002; Pack et al., 2006).

The extent to which $\mathrm{N}$ availability affects different physiological and agronomic traits of a crop species depends on the ability of the crop species to take up $\mathrm{N}$ from the soil and utilize it for the production of proteins and other essential N-containing components and therefore implicitly depends on the NUE of the crop species. 


\section{$\mathrm{N}$ use efficient plants}

Plants use three main strategies to use the available resource efficiently or to survive in irregular resource availability conditions. The first strategy is specialization: a genotype has adapted optimally to a specific environmental situation (low $\mathrm{N}$ environment-specific efficient or high $\mathrm{N}$ environment-specific efficient strategy); the second one is generalization: a genotype expresses moderate relevance for most environments, and the third one is phenotypic plasticity: the potential of the genotypes to express different phenotypes in different environments (Fritsche-Neto et al., 2012). Given the above three nutrient use strategies, farmers cultivating their crops under varying conditions including both stress and ideal conditions do not only need varieties that can give reasonable yield under low $\mathrm{N}$ stress conditions, but that also respond to ideal conditions with a sufficient yield increment (phenotypic plasticity). However, in most cases, phenotypic plasticity has a high negative association with yield stability (Bradshaw 2006), and stable varieties typically have low plasticity (less yield difference between most environments). Tolerant genotypes usually give moderate yield whether it is under ideal growing conditions or marginal conditions (under permanent stress) (Cruz et al., 2004). The productivity of these "generalized" genotypes is higher in resource poor environments, but under non-limiting environmental conditions, the yield increment is relatively low. Conversely, high-yielding crops under abundant $\mathrm{N}$ availability often show high plasticity, and therefore relatively low yields under N-limiting conditions. Limiting the trade-off between phenotypic plasticity and high yields under both low and high $\mathrm{N}$ is the challenge that breeders face in developing cultivars that are stable and high yielding under varying conditions. This trade-off also exemplifies the need to select for NUE and NUE traits under both high and low $\mathrm{N}$ conditions.

\section{$\mathrm{N}$ limitation adaptation mechanisms in plants}

At field conditions, plants are exposed to N-limiting situations due to several environmental factors like soil erosion, leaching, volatilization and microbial consumption. Therefore, adaptation to N-limiting conditions is an important survival strategy for plants to complete their life cycle effectively and give progenies. These adaptations include reduction in growth and photosynthesis, remobilization of $\mathrm{N}$ from old organs to actively growing ones, and the accumulation of anthocyanins (Ding et al., 2005; Diaz et al., 2006). N shortage results in marked reduction of plant photosynthesis in several crops, and the reduction is substantial, because more than half of the total leaf $\mathrm{N}$ is allocated to the photosynthetic apparatus (Makino and Osmond, 1991). Photosynthetic capacity and total amount of $\mathrm{N}$ per unit of leaf area are 
often positively correlated (Sage and Pearcy, 1987; Walcroft et al., 1997). N deficiency strongly affects photosynthesis, sugar metabolism, and /or carbohydrate partitioning between source and sink tissues (de Groot et al., 2003; Scheible et al., 2004). There is tangible evidence that $\mathrm{N}$ deficiency induces a sink constraint within the plant due to reduced growth, and photosynthesis is reduced to balance carbon assimilation to the reduced sink and low $\mathrm{N}$ availability (Paul and Foyer, 2001). N deficiency was shown to result in carbohydrate accumulation (sugars and starch) in the leaves, higher levels of carbon allocation to the roots and an increase in the root-to-shoot biomass ratio (Scheible et al., 2004; Remans et al., 2006).

Generally, plants constantly sense the changes in their environment, and when mineral elements are scarce, they usually allocate a higher proportion of their biomass to the root system, and this response is a result of metabolic changes in the shoot and an adjustment of carbohydrate transport to the roots (Lawlor et al., 2001). All these physiological and morphological changes are genetically and environmentally controlled, thus to develop $\mathrm{N}$ efficient crop varieties under $\mathrm{N}$ deficient conditions, knowledge about the genetics of NUE and NUE related traits is vital.

\section{The genetic basis of NUE}

NUE is a complex agronomic trait controlled by a large number of genes. The recent advancements in quantitative genetics have provoked a number of research groups to exploit the genetic differences of NUE in a focused manner. The association of physiological and agronomic traits with molecular markers is vital to infer the genetic basis of complex traits like NUE to determine the genes underlying the traits (Prioul et al.,1997; Hirel et al., 2007). The key organizational elements of the $\mathrm{N}$ assimilation pathway in higher plants are well recognized. Nitrate or ammonium uptake signifies the first step in this pathway, and a number of $\mathrm{N}$ transporters have been identified (Orsel et al., 2002). The enzymes nitrate reductase (NR) and nitrite reductase (NiR) are involved in the reduction process of nitrate to nitrite and nitrite to ammonium, respectively (Meyer and Stitt, 2001). The ammonium produced by this primary assimilation process is then incorporated in organic molecules by the glutamine synthetase (GS) or the glutamate synthase (GOGAT) pathway (Hirel and Lea, 2001). 


\section{Sources of organic and inorganic $\mathrm{N}$}

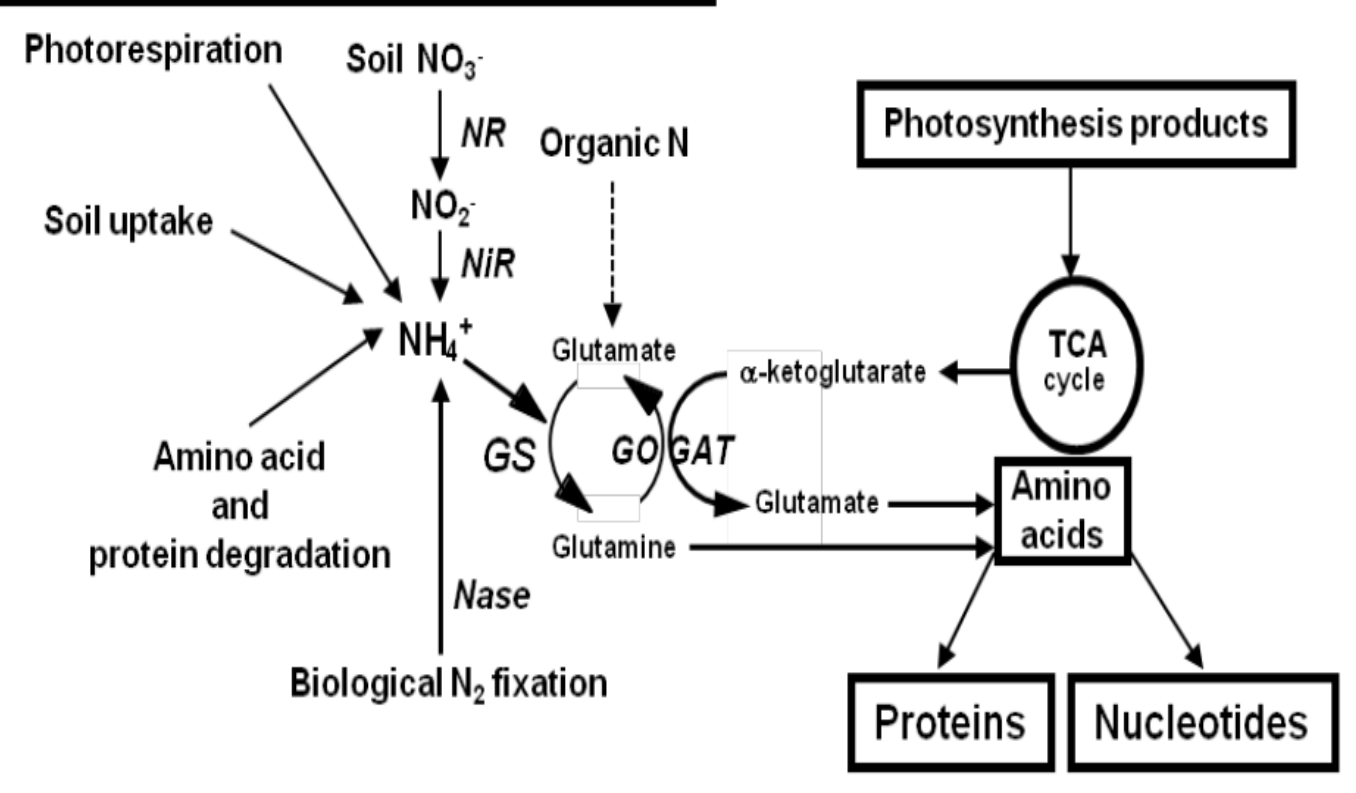

Figure 2. Main reactions involved in nitrogen assimilation in higher plants. The main enzymes involved in nitrogen assimilation are indicated in italics: $N R=$ nitrate reductase; $N i R=$ nitrite reductase; Nase $=$ nitrogenase; $G S=$ glutamine synthetase; GOGAT = glutamate synthase. Ammonium is incorporated into organic molecules in the form of glutamine and glutamate through the combined action of the two enzymes GS and GOGAT. Carbon originating from photosynthesis through the tricarboxylic acid cycle (TCA cycle) provides the $\alpha$-ketoglutarate needed for the reaction catalyzed by the enzyme GOGAT. Amino acids are used for the synthesis of proteins, nucleotides and other N-containing molecules (adapted from Hirel et al., 2011).

In order to identify the main regulatory and functional genes involved in regulation of the $\mathrm{N}$ assimilation pathway that can be marked for genetic mapping and tested for linkage with the trait, information on the biochemical and signaling pathways is required (Werij et al., 2007; Appleford et al., 2005). GS1 might be a key component of plant NUE and yield, whereas the physiological function of GS2 associated with NUE still needs to be identified (Bernard et al., 2009; Bernard et al., 2008). In addition to the identification of genes that regulate GS1 and GS2, researchers have identified QTLs for NUE and related physiological and agronomic traits in a number of crops. Obara et al., (2001) identified QTLs associated with NUE and determined their co-localization with glutamine synthetase1 (GS1) and glutamate synthetase NADH-GOGAT in rice. Many QTLs for agronomic traits associated to NUE and yield have been detected in the chromosomal regions surrounding GS2 and these may be important for breeding wheat and rice varieties with improved agronomic performance and NUE (Wu and Luo 1996; Obara et al., 2001; Yamaya et al., 2002; Fontaine et al., 2009). In a maize study, QTLs for yield (and its components) and QTLs for GS enzyme activity were identified in the same region, both of which co-localized with genes encoding cytosolic GS (Hirel and Lea, 2001). 
In potato, a wide range of phenotypic variation was observed for NUE among wild accessions and commercial cultivars, however efforts to understand the physiological and genetic basis for differences in NUE among cultivars are generally limited. Ospina (2016) tried to assess the genetic variation of potato for NUE under low and high $\mathrm{N}$ conditions using molecular markers and as a result both $\mathrm{N}$ level dependent and independent QTLs were detected for most canopy development traits of potato. Quantification of genetic diversity of the available genetic resources for their NUE and NUE related traits using biometrical tools and molecular markers is crucial to understand the genetic basis of NUE in potato.

\section{Breeding for NUE}

Nitrogen availability is crucial to obtain high yields in potato. Improvement of NUE in potato can reduce the nitrogen input required, consequently economic gain will be increased and environmental pollution due to $\mathrm{N}$ loss will be reduced. Although genetics and breeding of potato uses advanced approaches to identify the genes required for the genetic improvement of potato, the tetrasomic inheritance, high heterozygosity and self-incompatibility barriers hinders the genetic improvement of the potato crop (Jansky and Peloquin, 2006; Lindhout et al., 2011). On the other hand, these reproductive constraints can be compensated by other unique features of potato; namely the use of unreduced gametes ( $2 \mathrm{n}$ gametes) and the possibility of crossing diploids with tetraploids. These attributes contributed a lot to the evolution and genetic variation of potato (den Nijs and Peloquin, 1977; Peloquin et al., 1989; Carputo and Barone, 2005; Ortiz et al., 2005)

A wide range of phenotypic variation in NUE has been reported in commercial potato cultivars, clonal selections, and accessions of wild potato species (Errebhi, Rosen, and Martin et al., 1998, Errebhi et al., 1999; Zebarth et al., 2004a; Sharifi et al., 2007). This genetic variability within the cultivated potato and its wild relatives can be exploited by breeders to improve the NUE of the crop. However, the observed variation (phenotypic variation) is the interaction effect of genotype with environment, and to determine the independent effect of these factors, the total phenotypic variation should be partitioned into heritable and nonheritable components using suitable genetic parameters. According to Gopal (1999), genetic parameters and trait associations provide information about the expected response of different traits to selection and these aid in developing optimal breeding strategies. However, it may be challenging to improve such complex traits by direct selection. Due to this fact, the efficiency of selection for complex traits in low $\mathrm{N}$ environments may be improved through selection for correlated secondary traits (Blum, 1988; Hamawaki et al., 2012). Traits related to N uptake efficiency and $\mathrm{N}$ utilization efficiency have been suggested as a selection criteria for the 
improvement of NUE. In potato, NUE is highly correlated with canopy cover traits and maturity, and it was generally higher for later maturing potato cultivars than for early maturing ones (Zebarth et al., 2004a; Tiemens-Hulscher et al., 2012 ; Ospina et al, 2014). With this information, one can identify the best selection strategy of superior genotypes for a targeted trait breeding program. Successful breeding for complex traits like NUE does not only require information about the effective agronomic and physiological traits that contribute to NUE improvement, but their heritability and genotype-by-environment interaction should be established, and suitable selection tools for these traits of interest need to be available. Genetic mapping approaches like quantitative trait loci (QTL) and association mapping using molecular markers and genomics tools are nowadays essential tools in dissecting genetics of a complex trait like NUE.

\section{QTL mapping}

Biometrical approaches of quantitative genetics that deploy the phenotypic and pedigree information examine the joint effects of all genetic factors, and cannot distinguish the effects of individual loci. To dissect the complexity of quantitative traits in component loci and identify the genetic factors that influence quantitative traits, QTL analysis is a powerful tool (Doerge, 2002). QTL analysis with a wide range of molecular markers provides opportunities not only for the identification of QTLs that determine the phenotypic value of a particular trait, but also for the analysis of the relationships between traits (Lebreton et al., 1995; Simko et al., 1997). In the last two decades many QTL analysis studies have been published on different traits of potato, such as flower color, foliage maturity, tuber skin texture, dry matter content, specific gravity and yield (McCord et al.,2011), yield, agronomic and quality traits (Bradshaw et al.,2008), tuber yield and starch content (Schafer-Pregl et al.,1998, Werij et al., 2012), tuber dormancy (van den Berg et al., 1996), tuber shape (Van Eck et al., 1994b), tuber skin color (Gebhardt et al.,1989), tuber flesh color (Bonierbale et al., 1988), shoot fresh weight, tuber number, tuber weight and root length under drought stress and recovery conditions (Anithakumari et al., 2011; 2012) and NUE related canopy cover traits under contrasting $\mathrm{N}$ regimes (Ospina, 2016). However, knowledge about the genetic dissection of NUE in potato is still limited and hardly any QTL has been published for NUE and NUE related traits of potato. Moreover, selection for NUE and improvement of NUE in potato under marginal conditions targeted at regions where subsistence farmers rely on potato yields with little or no $\mathrm{N}$ input (like in Ethiopia) is hardly or not at all explored. 


\section{Association mapping}

Traditional QTL mapping has been used as a method to understand the genetic regulation of polygenic traits and has been important for detecting QTLs in different crop species in the last 20 years. However, association mapping is rapidly becoming the method of choice to identify QTL and to find molecular markers associated with complex traits. This is mainly due to its broad allele coverage, high resolution and cost effectiveness, as it does not require the creation of mapping populations, and can be used to target multiple traits (Ross-Ibarra et al., 2007; Stich et al., 2006). This methodology avoids some limitations of QTL mapping and it is often even a preferred alternative for genetic studies. Association analysis has the potential to detect QTL associated with the desired trait and, when a large number of markers are available for a large set of genotypes, even to identify the causal polymorphisms within a gene that are responsible for the difference in two alternate phenotypes (Gupta et al., 2005). In association mapping the resolution of the QTL mapping is high as often only closely linked alleles are in linkage disequilibrium (LD) due to long history of many generations of recombination (Ingvarsson and Street, 2011). Moreover, association mapping deals better than bi-parental mapping with non-inbred crop species like potato that has complex tetrasomic inheritance ( $\mathrm{Li}$ et al., 2010b). Association mapping studies in potato have been published for disease resistance and quality trait mapping. Gebhardt et al., (2004) mapped late blight resistance and plant maturity, Simko et al. (2004) Verticillium resistance, Malosetti et al., (2007) late blight resistance and D'hoop et al. $(2008,2014)$ used association mapping for agro-morphological and quality traits. The genetic desiccation of NUE and NUE related traits in potato using association mapping method has been reported by Ospina (2016). Various N level dependent and $\mathrm{N}$ level independent marker-trait associations were identified for many NUE related traits; however, the consistency of the identified marker-trait associations may be affected by the genotypes involved in each study and the variation of the environment in which the trial was conducted.

\section{Genotype-by-environment: the challenge in selecting for NUE}

NUE is a complex quantitative trait, and such traits are often controlled by multiple genes, with each gene having a small effect. In addition, the phenotypic evaluation of the NUEassociated traits is strongly influenced by environmental variation. Genotypes tested in different production systems, locations and years usually have significant fluctuation in yield due to the response of the genotypes to biotic as well as abiotic environmental effects (Kang, 2002). This so-called genotype-by-environment (GE) interaction affects the breeding 
progress, because it complicates the detection of superior genotypes across environments (Ebdon and Gauch, 2002). It also results in low correlation between phenotypic and genotypic values, causing reduced selection efficiency. This leads to estimations of heritability in one environment that cannot be easily translated to other environments, and to poor predictions of genetic advance (Comstock and Moll, 1963). Therefore, determining the degree of GE interaction for a trait of interest facilitates more reliable selection and creation of test programs for the development of superior varieties.

Evaluation of crop varieties for their NUE potential is not common in most crop breeding programs. Most breeding programs are conducted in environments where inputs like nitrogen (N) fertilizers are highly controlled to minimize the nutrient deficiency and environmental variability. However, cultivars developed under high $\mathrm{N}$ input condition may not perform well under low $\mathrm{N}$ input conditions. Sofar no work has been done on the suitability of test environments and stability of potato cultivars for NUE under different production conditions including low $\mathrm{N}$ level and high $\mathrm{N}$ level as part of the environment.

\section{Objectives and scope of the thesis}

The research described in this thesis was initiated to enhance our understanding of the genetic basis of NUE and NUE-related agronomic and physiological traits in potato when grown in Ethiopia. We have studied genetic diversity for NUE of cultivated potato from Ethiopia and Western Europe under Ethiopian growing conditions, and identified the joint and individual effects of QTLs on our target traits in these cultivars as well as in a diploid mapping population. Finally we have addressed GxE interaction considering $\mathrm{N}$ level as part of environment and NUE as a target trait, which will help us to exploit genetic and environmental resources more efficiently and identify ideal test environments and superior genotypes for NUE improvement at different $\mathrm{N}$ fertilizations levels and production systems.

In Chapter 2, the extent and pattern of genetic diversity and association among desired agronomic and physiological traits that affect NUE of potato under low and high $\mathrm{N}$ availability are extensively evaluated and discussed. Cluster analysis, estimation of genetic parameters, correlation and path coefficient analysis are used as tools to assess the genetic diversity and association of traits. Potential agronomic and physiological traits that show strong positive correlations with NUE and can be used as potential secondary traits for indirect selection and genetic improvement of NUE are identified and discussed. Chapter 3 is dedicated to finding genetic factors contributing to NUE under low and high $\mathrm{N}$ conditions in a diploid mapping population $(\mathrm{CxE})$. The population was grown in Ethiopia, at several 
locations and in two seasons, and significant variation among genotypes was observed for NUE and NUE related traits both under low and high $\mathrm{N}$ condition. In order to identify potential QTLs that contribute to NUE of potato under low and high N conditions, a SNP marker-rich integrated linkage map of $\mathrm{CxE}$ was used. In Chapter 4, European commercial potato cultivars and progenitor lines genotyped using infinium SNP array technology markers were used for association mapping. The population was grown in multiple locations and years, and phenotyped, and marker trait associations and multi-trait QTLs were identified.

In Chapter 5, Genotype x Environment interaction of the response to low and high $\mathrm{N}$ levels is described in European commercial potato cultivars and progenitor lines, along with Ethiopian cultivars. Eight environments representing low and high $\mathrm{N}$ level combined with rain fed and irrigation production conditions were considered as test environments. As a result, two mega environments, suitable cultivars in each mega environment and ideal testing environments within the mega-environment were identified. The discriminating power and representativeness of test environments, and the mean performance and stability of the genotypes in NUE within a mega-environment are extensively reviewed and discussed. In the general discussion (Chapter 6), the results from Chapter 2 to Chapter 5 are summarized, and examined for their implications on the potato breeding strategy for the improvement of NUE. The prospects of breeding for NUE in potato especially for low $\mathrm{N}$ conditions in relation to our findings is discussed. 
Chapter 2

\section{Genetic diversity of potato cultivars for nitrogen use efficiency under contrasting nitrogen regimes}

Baye Berihun Getahun ${ }^{1,2}$, Molla Mekonen Kassie ${ }^{3}$, Richard RGF Visser ${ }^{1,2}$, C. Gerard van der Linden ${ }^{1,2}$

${ }^{1}$ Graduate School of Experimental Plant Sciences, Wageningen University \& Research PO Box 386, 6700 AJ Wageningen, The Netherlands

${ }^{2}$ Plant Breeding, Wageningen University \& Research, PO Box 386, 6700 AJ Wageningen, The Netherlands;

${ }^{3}$ Amhara Agricultural Research Institute, PO Box 08, Bahirdar, Ethiopia 


\begin{abstract}
The importance of proper $\mathrm{N}$ fertilizer use gets more critical as environmental and economic concern over $\mathrm{N}$ fertilizer use intensity in crop production increases. To assess the genetic diversity for nitrogen use efficiency (NUE) and related traits in potato, a total of ninety seven (eighty-eight Dutch and nine Ethiopian) cultivars were evaluated at two nitrogen levels (40kg $\mathrm{ha}^{-1}$, and $120 \mathrm{~kg} \mathrm{ha}^{-1}$ ) for 18 quantitative traits in Debre-Tabor and Injibara, (Ethiopia) in the 2013 rainfed season. Plant height, maximum canopy cover (Vmax), area under the canopy curve (AUC), lower leaf chlorophyll content, tuber yield componets and NUE were significantly affected by $\mathrm{N}$ levels across locations. NUE, tuber dry matter \%, and days to maturity had higher mean values at low $\mathrm{N}(\mathrm{LN})$ than at high $\mathrm{N}(\mathrm{HN})$ in both locations. The tuber yield reduction due to $\mathrm{N}$ limitation was significant, and tuber number plant ${ }^{-1}$ reduced total tuber weight more than average tuber weight especially in Injibara. The cluster analysis grouped the Ethiopian cultivars into five and four clusters at low and high conditions, respectively. The Dutch cultivars grouped in to five clusters at low $\mathrm{N}$ input and seven clusters at high $\mathrm{N}$ input and the genetic distance between most clusters was significant. High phenotypic coefficient of variation, genotypic coefficient of variation, heritability and genetic advance as a percent of mean were observed for tuber number plant ${ }^{-1}$, average tuber weight and NUE in Debre-Tabor and plant height, tuber number plant ${ }^{-1}$ and average tuber weight in Injibara under both $\mathrm{N}$ regimes. Strong phenotypic correlation coefficients were observed between NUE and tuber number plant ${ }^{-1,}$ days to maturity, tuber dry matter $\%$, Vmax and AUC under both low and high $\mathrm{N}$ input conditions. Dutch cultivars showed rapid initial canopy development and matured early compared to the Ethiopian cultivars at both $\mathrm{N}$ levels and locations. Higher NUE values were observed for late maturing potato cultivars at both $\mathrm{N}$ rates. Our study indicates that potato cultivars can be exploited for NUE improvement through improving and pyramiding of component traits at both low and high $\mathrm{N}$ levels.
\end{abstract}

Keywords: Genetic diversity, correlation, NUE, potato 


\section{Introduction}

Potato is the $1^{\text {st }}$ non-grain food crop in the world, with an important contribution to the diets and livelihoods of millions of people globally. It is well-recognized by the United Nations (UN) as staple food in contributing to the Millennium Development Goals (MDG) for its potential to reduce poverty and improve food security (Bradshaw, 2009). However, the productivity potential is highly dependent on nitrogen fertilizer level applied and/or available in the soil. With high $\mathrm{N}$ fertilizer prices, the return of $\mathrm{N}$ input is low due to low uptake and utilization efficiency of the crop. The high costs and low returns of fertilizer use particularly burdens smallholder farmers in developing countries, and force them to grow their crop under suboptimal N supply (Lafitte and Edmeades, 1994). Application of surplus nitrogen beyond the utilization capacity of the crop is affordable in high input agriculture and maximizes yield, but leads to nitrate pollution of ground water (Bertin and Gallais, 2001). In developed countries $50-70 \%$ of the nitrogen provided to the soil for crop production is lost (Hodge et al., 2000). Most commercial potato cultivars are grown with high $\mathrm{N}$ fertilizer levels often combined with irrigation, resulting in high reduction of nitrogen utilization efficiency (Zebarth et al, (2004a), highly contributing to $\mathrm{N}$ leaching and ground water contamination (Hill, 1986; Richards et al., 1990). Significant emissions of nitrous oxide (a greenhouse gas) were also measured from potato fields (Flessa et al., 2002). Collectively, this makes a compelling case for improving $\mathrm{N}$ fertilizer use in agricultural crops in developed as well as developing countries; to avoid nitrate pollution and to retain a sufficient profit margin, cultivars that are tolerant to low levels of nitrogen input are desirable (Bänziger et al., 2001).

Identification of crop plants that show exploitable variation for the traits of interest is the first step of any successful crop breeding program. Genetic diversity studies contribute to the understanding of genetic relationships among populations and consequently lead to specific heterogeneous groups which are essential for the identification of parents for hybridization (Mostafa et al., 2011). Knowledge about the level of genetic diversity can aid with the introgression of desirable genes from diverse genepools into the available germplasm (Thompson and Nelson, 1998). To this end, a better understanding of the genetic diversity, the different physiological processes involved and the underlying genetic relationships in plants grown under low and high $\mathrm{N}$ input regimes could give a better insight for breeding programs on nitrogen use efficiency (NUE). Genetic variation in potato germplasm offers opportunities to improve potato by combining favorable traits contributing to yield under low $\mathrm{N}$ input conditions. Significant variation in biomass partitioning and $\mathrm{N}$ uptake efficiency at low and 
high $\mathrm{N}$ environments was observed among potato cultivars, hybrids and wild species (Errebhi et al., 1999). Differences in NUE under high and low $\mathrm{N}$ input regimes were shown to be strongly associated with maturity type (Tiemens-Hulscher et al., 2012). Late maturing potato cultivars recorded higher NUE values than early maturing ones (Zebarth et al., 2004a, Ospina et al., 2014). However, the genetic base of NUE in potato is still poorly understood. Therefore, it is important to have genetic diversity information of commercial cultivars in NUE and related traits in order to use them as germplasm source for potato NUE breeding programs.

Genetic parameters and trait associations provide information about the expected response of different traits to selection and help in developing an optimal breeding strategy (Gopal, 1999). In potato, many authors reported medium to high genetic parameter estimates. Moderate genotypic coefficient of variation (GCV) and high phenotypic coefficient of variation (PCV) values were reported for plant height, leaf area, tuber specific gravity and tuber dry matter (Regassa and Barasavaj, 2005; Desai and Jaiminis, 1997). High heritability combined with high genetic advance was also recorded for tuber yield plant ${ }^{-1}$, tuber number plant $^{-1}$ and average tuber weight (Regassa and Barasavaj, 2005). However, genetic parameter estimates may vary from population to population as well as from environment to environment. Accordingly, when estimating genetic parameters, one should also consider the population represented in the experiment and the environmental condition where the experiment was conducted in (Dudely and Moll, 1969; Nyquist, 1991). Therefore, information on the genetic parameters of potato NUE and related agronomic and physiological traits grown both under low and high N conditions is vital for NUE improvement in potato. Similarly, the interrelation of NUE with other physiological and agronomic traits is important for designing an effective breeding program. So far, information on the genetic parameters of different agronomic and physiological traits involved in NUE and the underlying genetic relationships of these traits in potatoes is limited. The objective of this study was: (i) To assess the extent and pattern of genetic diversity for NUE of Western potato cultivars compared to Ethiopian cultivars (ii) To estimate genetic parameters and association among desired traits that affect NUE of potato under low and high $\mathrm{N}$ availability. 


\section{Materials and methods}

\section{Plant materials}

Potato tubers of eighty-eight Dutch cultivars provided by HZPC Holland BV and nine Ethiopian varieties were used in the experiment. The Dutch cultivars are common cultivars in the European potato market, used for different purposes. From the Ethiopian set, Agerie and Ater-Ababa are traditional cultivars that are relatively tolerant to most biotic as well as abiotic stresses. Other varieties originated from the International Potato Center (CIP) and were released by different research centers in Ethiopia for their late blight resistance, tuber yield and wide adaptability in different parts of the country. See Suppl Table 1 for a full list of the used varieties.

\section{Field trials and experimental design}

The experiment was conducted at two different sites in North-western Ethiopia: Injibara and Debre-Tabor, which represent the major potato growing areas in this part of the country. Injibara is located at $10.85^{\circ} \mathrm{N}$ latitude and $36.80^{\circ} \mathrm{E}$ longitude. The area receives about $2300 \mathrm{~mm}$ average rainfall per year with average temperature of $8^{\circ} \mathrm{C}$ (night) and $22^{\circ} \mathrm{C}$ (day). The soil at the Injibara site is Acrisol with a $\mathrm{pH}$ of 4.8, and is of very strongly acidic nature. This soil acidity normally originates from the high amount of rainfall in the area, which is associated with heavy leaching of the top soil nutrients. Debre-Tabor is situated at an elevation of 2650 masl with $11.89^{\circ} \mathrm{N}$ longitude and $38.04^{\circ} \mathrm{E}$ latitude. The average night and day temperature is $12^{\circ} \mathrm{C}$ and $23^{\circ} \mathrm{C}$, respectively, with average rainfall of $1500 \mathrm{~mm}$ per year. The soil at the Debre-Tabor site is Luvisol of $\mathrm{pH}$ 5.2. The experiment was laid out in a splitplot arrangement with two replications, where the main plots were allocated to the low and high $\mathrm{N}$ rates $\left(40 \mathrm{~kg} \mathrm{ha}^{-1}\right.$, and $\left.120 \mathrm{~kg} \mathrm{ha}^{-1}\right)$ and the sub-plot to the genotypes. Each subplot was planted in a single row consisting of 10 tubers, planted at a recommended inter- and intra-row spacing of $0.75 \mathrm{~m}$ and $0.30 \mathrm{~m}$ respectively, and each subplot was bordered by a reference potato cultivar. Pest and disease management, weeding and ridging and other cultivations were carried out as recommended and when required. 
Table 1. Chemical properties of soils of the testing sites in Debre Tabor and Injibara.

\begin{tabular}{|c|c|c|c|c|c|c|c|c|c|c|c|c|c|}
\hline \multirow[b]{2}{*}{ Sites } & \multirow[b]{2}{*}{$\begin{array}{l}\text { Soil } \\
\text { type }\end{array}$} & \multirow[b]{2}{*}{$\begin{array}{l}\text { Soil } \\
\text { depth } \\
(\mathrm{cm})\end{array}$} & \multirow[b]{2}{*}{$\begin{array}{l}\text { Soil } \\
\mathrm{pH}\end{array}$} & \multirow[b]{2}{*}{$\begin{array}{l}\text { Total } \\
\mathrm{N}(\%)\end{array}$} & \multirow[b]{2}{*}{$\begin{array}{l}\text { Organic } \\
\text { carbon } \\
\text { (OC) } \%\end{array}$} & \multirow[b]{2}{*}{$\mathrm{C} / \mathrm{N}$} & \multirow[b]{2}{*}{$\begin{array}{l}\text { Available } \\
\text { P (ppm) }\end{array}$} & \multicolumn{6}{|c|}{ Exchangeable Cations $\left(\mathrm{cmol}_{\mathrm{c}} \cdot \mathrm{kg}^{-1}\right)$} \\
\hline & & & & & & & & $\mathrm{Na}^{+}$ & $\mathrm{K}^{+}$ & $\mathrm{Ca}^{2+}$ & $\mathrm{Mg}^{2+}$ & $\mathrm{H}^{+}$ & $\mathrm{Al}^{3+}$ \\
\hline \multirow[t]{2}{*}{ D/Tabor } & Luvisol & $0-20$ & 5.2 & 0.08 & 3.2 & 40 & 3.7 & 0.6 & 0.5 & 9.9 & 2.1 & 0.04 & 0.2 \\
\hline & & $20-40$ & 5.5 & 0.07 & 2.6 & 37 & 3.4 & 1.1 & 0.5 & 11.0 & 2.2 & 0.02 & 0.2 \\
\hline \multirow[t]{2}{*}{ Injibara } & Acrisol & $0-20$ & 4.8 & 0.03 & 3.5 & 117 & 9.5 & 1.2 & 0.5 & 6.7 & 2.0 & 0.08 & 2.3 \\
\hline & & $20-40$ & 4.8 & 0.07 & 3.4 & 49 & 7.4 & 1.2 & 0.4 & 8.0 & 2.1 & 0.62 & 2.0 \\
\hline
\end{tabular}

Phosphorus and nitrogen sources were from the soil as well as from externally applied fertilizer. To determine the available nitrogen in the soil, composite soil samples were collected using a core sampling method at five locations at $0-20 \mathrm{~cm}$ and $20-40 \mathrm{~cm}$ depth before planting from each of the experimental sites and the available residual nitrogen in the form of $\mathrm{NO}_{3}{ }^{-}$and $\mathrm{NH}_{4}{ }^{+}$was extracted using a $\mathrm{KCl}$ extraction method. A total of 15 and $12 \mathrm{~kg}$ $\mathrm{ha}^{-1} \mathrm{~N}$ were recorded at Injibara and Debre-Tabor, respectively. The total amounts of N (40 and $120 \mathrm{~kg} \mathrm{ha}^{-1}$ ) were achieved by adding $\mathrm{N}$ to the soil in the form of urea and di-ammonium phosphate (DAP). Phosphorus fertilizer was applied following the recommendation for that area $\left(69 \mathrm{~kg} \mathrm{ha}^{-1} \mathrm{P}_{2} \mathrm{O}_{5}\right)$ in the form of DAP and tri-super phosphate (TSP). The whole P source was applied at planting while $\mathrm{N}$ application was split in two: a week after emergence and at early flowering.

\section{Trait measurements}

The traits were measured similarly at the two experimental locations (Debre-tabor and Injibara). Days to emergence (DTE) was the number of days from planting when $50 \%$ of the plants emerged; it was assessed daily until all the plots had more than 50\% emergence. Plant height $(\mathrm{PH})$ was measured as the distance in $\mathrm{cm}$ from the soil surface to the top of the canopy when $50 \%$ of the genotypes were flowering. Stem number plant ${ }^{-1}$ (SNPP) was the number of stems of a genotype counted before the plant canopy declined. Chlorophyll content (CC) was measured in the lower and upper part of two middle plants in a row and on two leaves of each plant. The readings for chlorophyll content was taken from the third or fourth leaf from the top of the plant for upper leaf chlorophyll content (UCC), and the second or the third leaf 
from the base of the plant for lower leaf chlorophyll content (LCC) using a SPAD-502 chlorophyll meter (Minolta Co., Ltd. Japan), when $50 \%$ of the genotypes were flowering.

Soil cover (SC) was assessed every five days starting from 30 days after planting using a $0.6 \mathrm{~m} \times 0.75 \mathrm{~m}$ frame with 100 grid squares, positioned over the same middle plants in a plot for each measurement. Squares filled with foliage for more than $50 \%$ were counted, and the percentage of filled grid squares was considered soil cover percentage (SC\%). Days to maturity (DTM) was determined as the number of the days from emergence to the day at which more than $90 \%$ of the plants in a plot attained physiological maturity $(90 \%$ of the haulm tissues brown). The assessment was carried out every day starting from the time that early varieties showed the first signs of maturity.

\section{Tuber traits}

Harvesting was carried out once, when the last cultivars reached physiological maturity, and eight plants per plot were harvested and used to evaluate tuber traits. Tuber number plant ${ }^{-1}$ (TNPP) was recorded as the number of tubers collected from a matured plant at harvest. Average tuber weight (ATW) was the ratio of the weight of tubers per plant and number of tubers per plant at harvest. Tuber yield plant ${ }^{-1}$ (TYPP) (the average tuber yield plant $^{-1}$ ) was calculated taking the tuber yield (fresh weight) of all harvested plants from a cultivar, divided by number of plants harvested.

Specific gravity (SG) was determined using the tuber specific gravity procedure of weight in air and under water (Murphy and Goven, 1959). In evaluating the SG of each variety, healthy and marketable-sized grade $(20 \mathrm{~mm}$ and above) tubers were selected randomly from each variety harvest. Then, tubers were cleaned, and weighed both in air and water following the procedure of Murphy and Goven (1959). Specific gravity values were computed using the following formula:

$$
S G=\frac{W_{1}}{W_{1}-W_{2}}
$$

where $\mathrm{SG}=$ specific gravity of the material, $\mathrm{W}_{1}=$ weight in air of the sample tuber, in $\mathrm{g}$ and $\mathrm{W}_{2}=$ Weight of the sample completely immersed in water, in g. Tuber dry matter \% (TDM\%) normally is determined as a ratio of dry tuber weight to fresh weight expressed in percentage; we determined TDM\% indirectly from SG using empirical conversion factors following the equation of Kleinkopf et al. (1987): solid (Dry matter \%) $=-214.9206+(218.1852$ x SG). 
Tuber dry weight (TDW) was estimated indirectly from specific gravity and tuber dry matter content in percentage, using the following formula:

$$
T D W=\frac{T D M \% * T F W}{100}
$$

Where TDW $=$ Tuber dry weight in $\mathrm{g}, \mathrm{TDM} \%=$ Tuber dry matter percentage, $\mathrm{TFW}=$ Tuber fresh weight in $\mathrm{g}$.

Nitrogen use efficiency (NUE) is typically calculated as the yield per unit of $\mathrm{N}$ resource available to the plant (Moll et al., 1982). However, the method of NUE determination depends on the crop species and the objective of the study. In our case NUE was determined as the tuber dry matter production, or dry weight of the tuber per unit of $\mathrm{N}$ supplied. In this study, NUE was determined as the tuber dry matter production, or dry weight of the tubers $\mathrm{ha}^{-1}$, per unit of $\mathrm{N}$ supplied ha ${ }^{-1}(\mathrm{~N}$ in the soil + applied $\mathrm{N})$.

\section{Data processing}

The beta thermal time for canopy cover assessment was calculated from the date of emergence for each experimental unit, using the sigmoid part of the beta function for determinate growth (Yin et al.,2003), and a cardinal temperature (with $5.5^{\circ} \mathrm{C}$ as a base temperature, $23.37^{\circ} \mathrm{C}$ as optimum, and $34.58^{\circ} \mathrm{C}$ as ceiling temperature) that determines the vegetative growth of potato (Khan 2012, Khan et al.,2013). Hourly temperature was collected from the Debre-Tabor weather station for Debre-Tabor and we used the local climate estimator (New_LocClim) in the FAO wave site metrological database for Injibara (FAO, 2014).

The model for canopy development was fitted using the soil cover data and beta thermal time for each assessment, with the NOLIN procedure of SAS (SAS, Institute inc, 2004) (Yin et al., 2003). The canopy cover dynamics in potato as quantified by the grid method typically followed a pattern that can be divided in three major growing phases of the crop, i.e building phase, maximum cover phase and declining phase, and the equations describing each phase of the curve are shown along with the initial values for each parameter in Khan (2012) and Khan et al. (2013). Estimated parameters with their standard errors were obtained after optimization. For each experimental unit we estimated the values of five model parameters (tm1, t1, t2, te and Vmax) according to Khan (2012) and Khan et al. (2013). The time (t) parameters: $\operatorname{tm} 1$ (inflection point in the build-up phase of the growth curve), $\mathrm{t} 1$ (time when 
the canopy cover reaches its maximum growth), $\mathrm{t} 2$ (time of onset of canopy declining), and te (time when canopy cover reach to zero) were expressed in thermal time/day (td). The last parameter Vmax is the maximum canopy cover value with percentage of soil cover as unit. Based on the canopy development curve model the following characters were calculated: $\mathrm{t} 2-\mathrm{t} 1$ (duration of maximum canopy cover in td), te-t2 (duration of senescing of the canopy), AP1(area under the curve for growth phase one in \% td), AP2 (area under the curve for growth phase two in \% td), AP3 (area under the curve for growth phase three in $\%$ td), and AUC (area under the curve for the entire crop growth cycle in \% td). The value of AUC represents the capacity of the crop to intercept solar radiation over the whole growing season (Vos 1995, 2009).

\section{Statistical analysis}

To estimate the variance components for each trait, two types of analysis of variance (ANOVA) were performed using software Genstat $16^{\text {th }}$ Edition. The first ANOVA was executed separately for each $\mathrm{N}$ level at each location following a randomized complete block design system using one-way ANOVA. The second ANOVA was performed for each location and across locations using the general linear model for split plot design with two $\mathrm{N}$ level treatments as main plot and the genotypes as sub-plot. Least significant difference (LSD) was used to separate the means at $1 \%$ and/or $5 \%$ level of significance. Clustering was carried out using SAS software version 9.3 based on the generalized $\mathrm{D}^{2}$ distances by an average linkage method of hierarchical clustering called Unweighted Pair Group Methods with Arithmeticaverage (UPGMA). Genetic distance within and between clusters was calculated using the generalized Mahalanobis's $\mathrm{D}^{2}$ statistics. The $\mathrm{D}^{2}$ is defined as: $D^{2} i j=(x i-x j) S^{-1}(x i-x j)$

where, $\mathrm{D}^{2} \mathrm{ij}=$ is the distance between two clusters $\mathrm{i}$ and $\mathrm{j} ; \mathrm{Xi}$ and $\mathrm{Xj}$ are the two vector means of the traits for $\mathrm{i}^{\text {th }}$ and $\mathrm{j}^{\text {th }}$ groups respectively, and $\mathrm{S}^{-1}$ is the inverse of the pooled covariance (Mahalanobis,1936). The $\mathrm{D}^{2}$ value obtained for pairs of clusters was considered as the calculated value of Chi-squared $\left(\mathrm{X}^{2}\right)$ and was tested for significance at the required level of probability against the tabulated values of $X^{2}$ for $p$ degrees of freedom, where $p$ is the number of characters considered (Singh and Chaudhary, 1985).

Regression and correlation analysis were carried out between the traits measured in each specific environment for traits that had a significant $\mathrm{F}$ test value. The variance components were computed using Genstat $16^{\text {th }}$ Edition. The phenotypic (PCV) and genotypic (GCV) coefficient of variations were determined using the method defined by Burton et al. (1953): 


$$
P C V=\frac{\sqrt{\text { Phenotypic variance }}}{\text { Population mean for the trait }} \times 100 \text { and }
$$

$$
G C V=\frac{\sqrt{\text { Genotypic var iance }}}{\text { Population mean for the trait }} \times 100
$$

Broad sense heritability was estimated from the total genetic variance using the method described by Falconer and Mackay (1996): $H^{2}=\sigma^{2} g / \sigma^{2} g+\sigma^{2} e$. The genetic advance (GA) expected under selection, assuming selection intensity of 5\% was calculated by the formula suggested by Johanson et al. (1955): $G A=K \cdot H^{2} \sigma p$

Where $\mathrm{k}=$ selection differential $\left(\mathrm{k}=2.06\right.$ at $5 \%$ selection intensity), $\mathrm{H}^{2}=$ broad sense heritability, and $\sigma p=$ phenotypic standard deviation.

To study the inter-relationships among measured traits and their direct and indirect contributions to NUE under low and high $\mathrm{N}$ fertilizer level, means of traits that revealed high genetic variation were subjected to correlation and path analysis (Dewey and Lu, 1959; Lal et al., 1997).

\section{Results}

\section{$\mathbf{N}$ level effects}

A summary of traits values under low and high $\mathrm{N}$ regimes at each location is presented in Table 2. The response to the $\mathrm{N}$ treatments were different from trait to trait. Most traits had higher mean values at high nitrogen (HN) than at low $\mathrm{N}(\mathrm{LN})$. NUE, tuber dry matter percentage (TDM\%) and days to maturity (DTM) were traits that showed higher mean values at $\mathrm{LN}$ than at $\mathrm{HN}$ in both locations. The mean values of curve-derived thermal time parameters tm1 (the inflection point, during the canopy building growth phase), t1 (the time point when the crop reaches maximum canopy cover level), t2-t1 (the duration when the crop retains its maximum canopy), t2 (the time point when maximum canopy cover start decline), and te (the time point canopy cover zero) showed small differences between low and high $\mathrm{N}$ conditions.

Low $\mathrm{N}$ availability caused a significant $(\mathrm{P} \leq 0.01)$ reduction $(23 \%$ in Debre-Tabor and $40 \%$ in Injibara) in potato maximum canopy cover (Vmax) (Table 2, Figure 1). Similarly, the area under the canopy curve for the entire crop growth cycle (AUC), representing the total light intercepted by a cultivar during the growth cycle, was significantly $(\mathrm{P} \leq 0.01)$ affected by low $\mathrm{N}$ availability. Among the three growing phases, the effect of $\mathrm{N}$ on area under the curve in the 
building phase (AP1) and area in the senescence phase (AP3) was considerable compared to area under the curve in phase two (AP2) especially in Injibara, while in Debre-Tabor the effect was higher in AP2 (Table 2). The extent of the N effect was also different between the two locations. AUC was reduced by $28 \%$ at Debre-Tabor and $37 \%$ at Injibara. Area under the curve in the building phase (AP1) was reduced by $25 \%$ at Debre-Tabor and $53 \%$ at Injibara, while area in phase two (AP2) and three (AP3) respectively were reduced by 46 and $21 \%$ in Debre-Tabor and 20 and 32\% in Injibara. The effect of $\mathrm{N}$ levels on days to maturity was not significant at both locations. The result indicate that $\mathrm{N}$ shortage substantially affected the area under the canopy curve parameters in all growing phases and in both locations, however the effect was different between locations. AUC, AP1 and AP3 were highly affected by low N in Injibara, while AP2 was highly reduced by low $\mathrm{N}$ in Debre-Tabor. The differences in response to $\mathrm{N}$ of these parameters at least partly reside in the opposite response of the maximum canopy duration ( $\mathrm{t} 2-\mathrm{t} 1)$ in Injibara compared to Debre-Tabor (increased in Injibara, while decreased in Debre-Tabor (Table 2).

Table 2. Trait means of 97 potato cultivars evaluated at high N level (HN) and low N level (LN) in Debre-Tabor and Injibara.

\begin{tabular}{|c|c|c|c|c|c|c|}
\hline \multirow[t]{3}{*}{ Traits } & \multicolumn{3}{|c|}{ Debre-Tabor } & \multicolumn{3}{|c|}{ Injibara } \\
\hline & \multicolumn{3}{|c|}{ N-levels } & \multicolumn{3}{|c|}{$\mathrm{N}$ levels } \\
\hline & $\mathrm{HN}$ & $\mathrm{LN}$ & $\begin{array}{l}\% \text { reduction } \\
(\mathrm{LN} \text { vs } \mathrm{HN})\end{array}$ & $\mathrm{HN}$ & $\mathrm{LN}$ & $\begin{array}{l}\% \text { reduction } \\
(\mathrm{LN} \text { vs } \mathrm{HN})\end{array}$ \\
\hline DTE & 13.5 & 13.1 & 4 & 13.9 & 13.0 & 7 \\
\hline PH & 44.2 & 36 & 19 & 27 & 16 & 42 \\
\hline SNPP & 4 & 4 & 4 & 4 & 4 & 4 \\
\hline Vmax & 69.5 & 53.3 & 23 & 62.0 & 37.1 & 40 \\
\hline $\mathrm{tm} 1$ & 7.9 & 8.1 & 2 & 9.6 & 10.6 & 10 \\
\hline $\mathrm{t} 1$ & 13.5 & 13.6 & 1 & 16.7 & 17.5 & 4 \\
\hline $\mathrm{t} 2$ & 17.7 & 16.6 & 5.7 & 21.5 & 24.0 & 11.3 \\
\hline t2-t1 & 4.2 & 3.0 & 27 & 4.8 & 6.5 & 35.4 \\
\hline te & 30.7 & 30.0 & 2 & 34.0 & 38.0 & 11.8 \\
\hline te-t2 & 13.0 & 13.39 & 3 & 12.5 & 14.3 & 14.6 \\
\hline
\end{tabular}




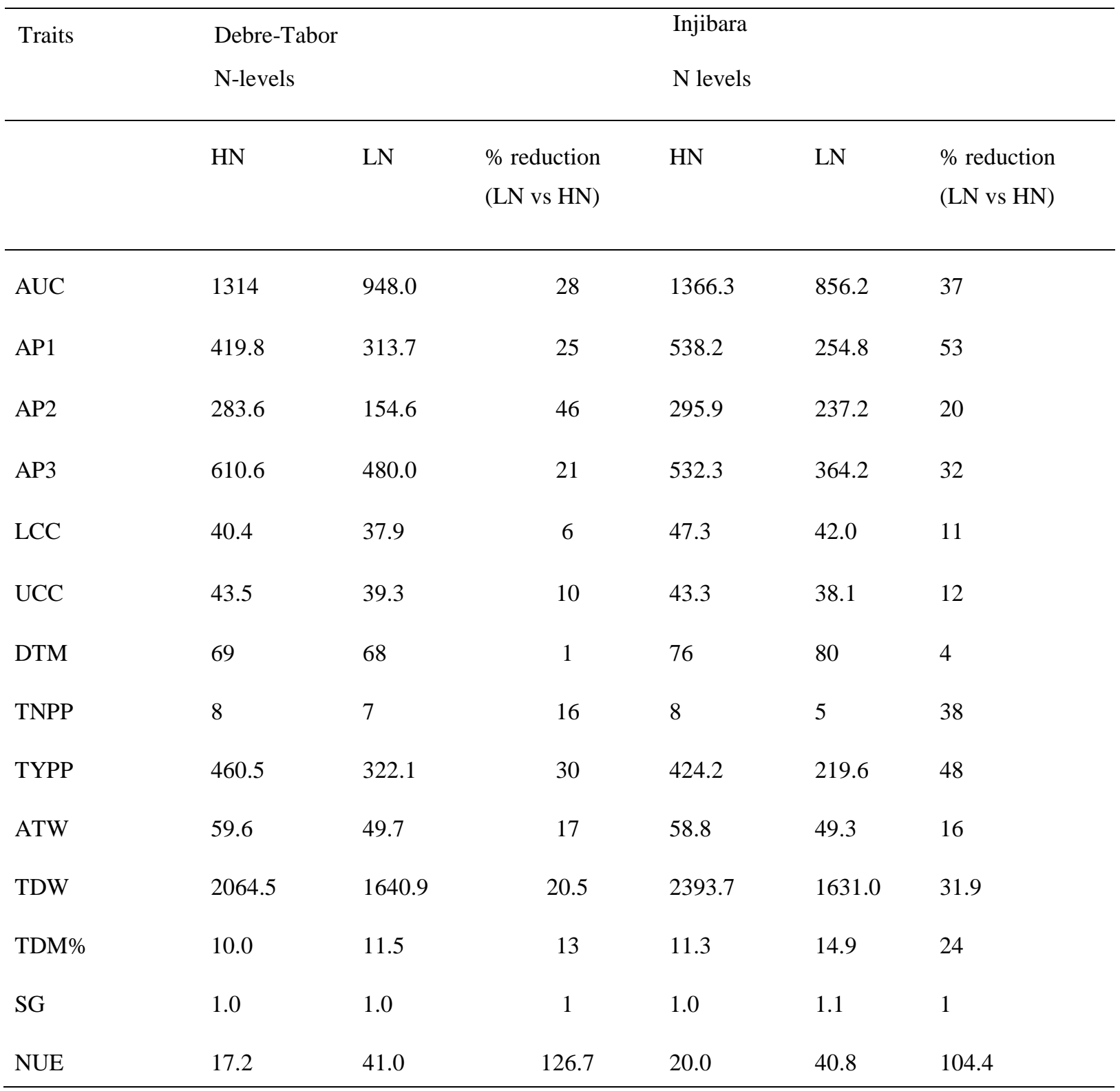

DTE $=$ Days to emergence, $\mathrm{PH}=$ Plant height $(\mathrm{cm}), \mathrm{SNPP}=$ Stem number plant $^{-1}$, Vmax $=$ Maximum canopy cover in $\%, \quad t m 1=$ Inflection point in thermal day (td), $\mathrm{t} 1=$ Canopy stabilized in td, $\mathrm{t} 2=$ Onset of canopy senesced in $\mathrm{td}, \mathrm{t} 2-\mathrm{t} 1=$ Duration for max canopy in $\mathrm{td}$, te $=$ Completely senesced canopy in $\mathrm{td}, \mathrm{AUC}=\mathrm{Total}$ area under the canopy in $\%$ td, AP1 $=$ Area for growth phase one in $\%$ td, AP2 = Area for growth phase two in \% td, $\mathrm{AP} 3=$ Area for growth phase three in $\% \mathrm{td}, \mathrm{LCC}=$ Lower leaf chlorophyll content (SPAD readings), UCC= Upper leaf chlorophyll content (SPAD readings), DTM= Days to maturity Tuber, TNPP $=$ Tuber number plant ${ }^{-1}$, TYPP $=$ Tuber yield plant ${ }^{-1}$ in $\mathrm{g}, \mathrm{ATW}=$ Average tuber weight in $\mathrm{g}, \mathrm{TDW}=$ Tuber dry weight in $\mathrm{kg} \mathrm{ha}^{-1}$, TDM\% $=$ Tuber dry matter $(\%), \mathrm{SG}=$ Specific gravity $\mathrm{g} \mathrm{g}^{-1}$ Nitrogen use efficiency $\mathrm{kg} \mathrm{kg}^{-1,} \mathrm{HN}=$ high $\mathrm{N}\left(120 \mathrm{~kg} \mathrm{ha}^{-1}\right)$, $\mathrm{LN}=$ low $\mathrm{N}\left(40 \mathrm{~kg} \mathrm{ha}^{-1}\right)$

The effect of $\mathrm{N}$ levels on tuber traits of the cultivars was signifcant. The reduction due to $\mathrm{N}$ shortage was considerable for average tuber weight (ATW), tuber number plant $^{-1}$ (TNPP), and total tuber yield plant $^{-1}$ (TYPP) at both locations. The TYPP was reduced by $30 \%$ in DebreTabor and $48 \%$ in Injibara. Of the two tuber yield components, TNPP was reduced by $16 \%$ in Debre-Tabor and 38\% in Injibara, while ATW was reduced by $17 \%$ in Debre-Tabor and 16\% in Injibara. This higher tuber yield reduction at Injibara may be related to the low $\mathrm{pH}$ of the 
soil. The effect of $\mathrm{N}$ for almost all traits was higher in Injibara than in Debre-Tabor, but not for NUE. NUE increased by $126.7 \%$ in Debre-Tabor and $104.4 \%$ in Injibara at low N compared to high $\mathrm{N}$ levels. TNPP was lower in Injibara compared to Debre-Tabor, and TDM\% was higher in Injibara.

\section{Location and cultivar effects}

The variation among cultivars was significant for all traits at both locations (Table 3). The effect of location was significant for most measured traits except ATW, NUE, te-t2 and AUC (Table 3). The non-significant variation between the two locations for AUC is due to the counterbalancing effect of higher maximum canopy cover (Vmax) in Debre-Tabor and higher cumulative thermal time for the growth period in Injibara (Figure 1). Potatoes in the Injibara trial matured later, and were harvested later as well. The duration for maximum canopy cover phase (t2-t1) was shorter compared to the other two phases in both Debre-Tabor and Injibara (Table 2).

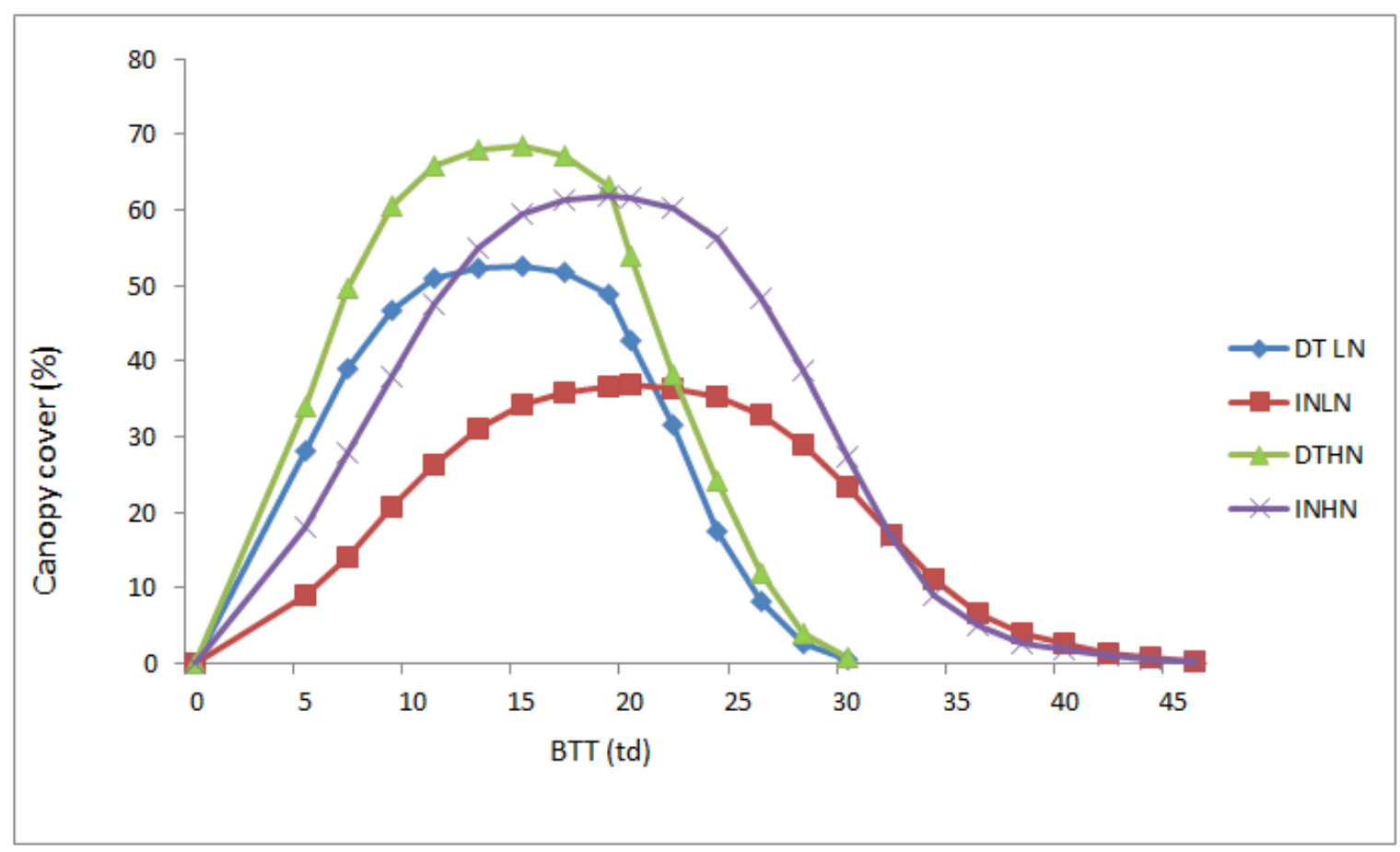

Figure 1. Canopy cover development at low and high $\mathrm{N}$ regime in Injibara and Debre-Tabor. INHN= Injibara high $\mathrm{N}, \mathrm{INLN}=$ Injibara low N, DTLN=Debre-Tabor low N, DTHN= Debre-Tabor high $\mathrm{N}$ and BTT= beta thermal time in ${ }^{0} \mathrm{C}$ day, $\mathrm{td}=$ thermal day 
Based on the maturity data collected in this experiment, we have classified our cultivars into an early, intermediate and late maturity group. The variation between the late maturity group and the intermediate and early maturity group was visible for most traits (including TYPP, TDM\% and AUC) under low and high $\mathrm{N}$ conditions in Debre-Tabor (Figure 2). The late maturity group cultivars had higher values for AUC as well as TYPP at both N levels in Debre-Tabor. In Injibara however, the variation among these maturity groups was lower, and even negligible for TYPP at both $\mathrm{N}$ levels. The late maturity group even had higher values for AUC compared to the early and intermediate maturity group (Figure 2).
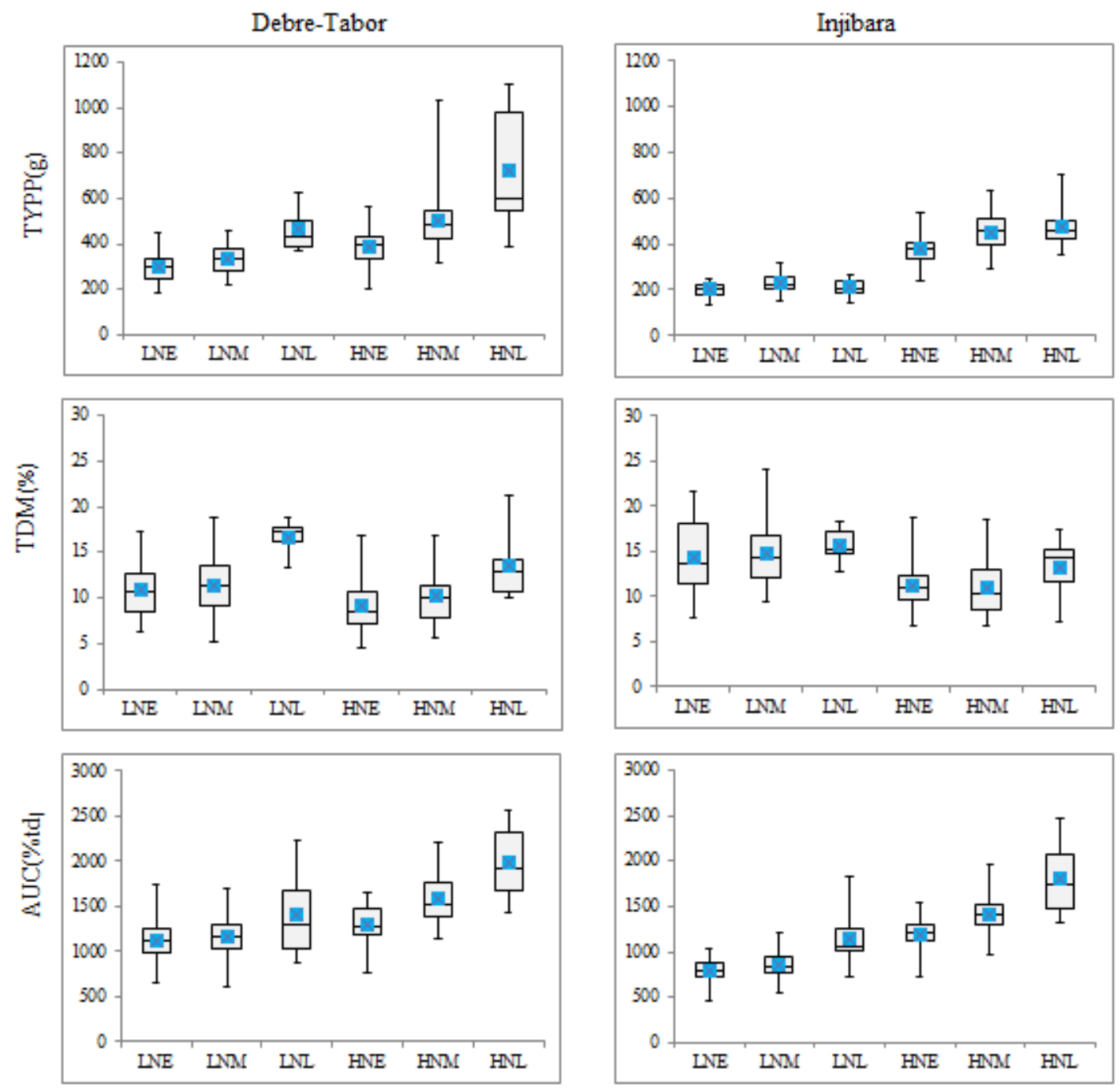

Figure 2. Box plots of selected traits to show the mean performance of cultivars in their maturity group combined with nitrogen levels in Debre-Tabor and Injibara. The grouping elements on the $\mathrm{x}$-axis are a combination of $\mathrm{N}$ levels and maturity groups. $\mathrm{LNE}=$ low nitrogen with early cultivars, $\mathrm{LNM}=$ low nitrogen with mid cultivars, $\mathrm{LNL}=$ low nitrogen with late cultivars, $\mathrm{HNE}=$ high nitrogen with early cultivars, HNM= high nitrogen with mid cultivars, $\mathrm{HNL}=$ high nitrogen with late cultivars. TYPP $=$ Tuber yield plant $^{-1}$, TDM $\%=$ Tubrt dry matter in \%, and AUC = Area under the canopy curve in \% thermal day $(\% \mathrm{td}) . \mathrm{LN}=$ low N $\left(40 \mathrm{~kg} \mathrm{ha}^{-1}\right)$, $\mathrm{HN}=$ high $\mathrm{N}\left(120 \mathrm{~kg} \mathrm{ha}^{-1}\right)$. 
Most Dutch cultivars were classified in the early and intermediate maturity group while most Ethiopian cultivars clustered in the late maturity group at both locations, suggesting that maturity is the main factor for the variation between the Ethiopian and the Dutch cultivars. To test this, Ethiopian and Dutch cultivars that clustered in the same (late) maturity group were compared, and this revealed that in the late maturity cluster, the Ethiopian cultivars performed better than the Dutch cultivars for AUC especially under high N conditions, but had lower tuber yields in Injibara (Figure 3), indicating that the Ethiopian cultivars in Injibara in particular were not able to translate the higher light interception capacity to higher yields.
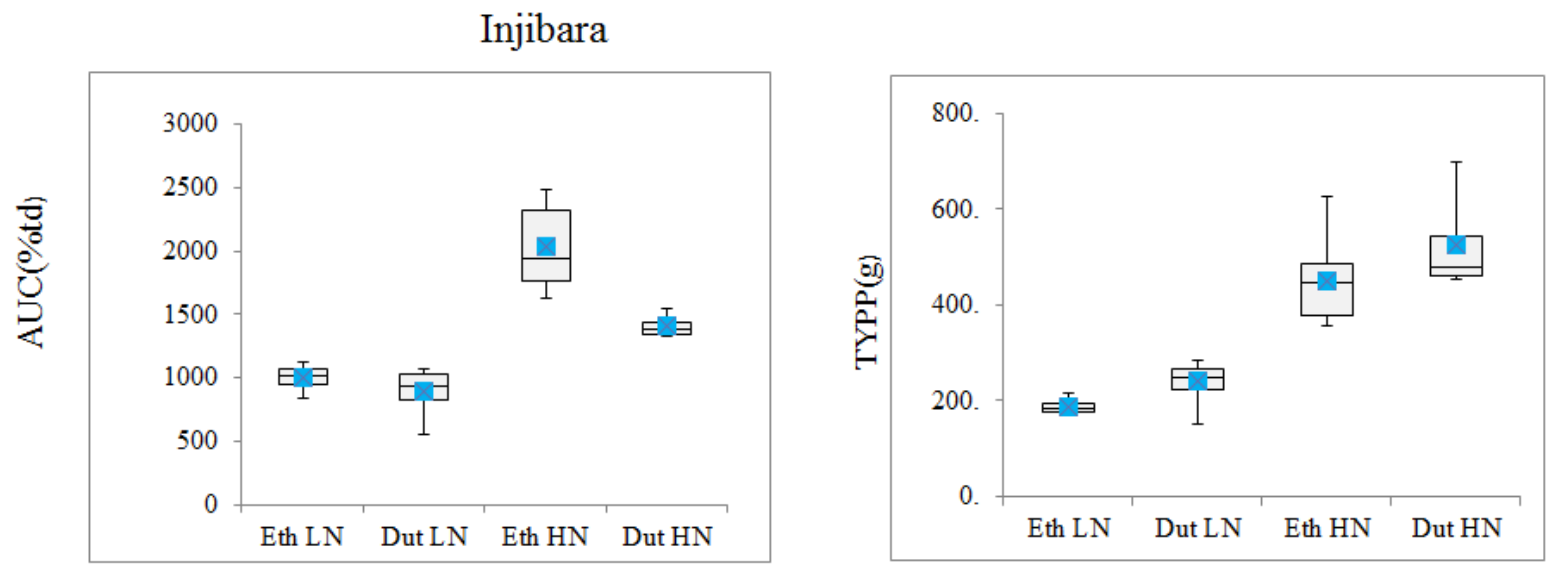

Figure 3. Box plots of tuber yield plant ${ }^{-1}$ and area under the canopy curve (AUC) to show the mean performance of the Ethiopian and Dutch cultivars in the late maturity group in Injibara. The grouping elements on the $\mathrm{x}$-axis are a combination of origin of cultivars and $\mathrm{N}$ levels. $\mathrm{LN}=$ low $\mathrm{N}\left(40 \mathrm{~kg} \mathrm{ha}^{-1}\right), \mathrm{HN}=$ high $\mathrm{N}\left(120 \mathrm{~kg} \mathrm{ha}^{-1}\right)$. Eth $\mathrm{LN}=$ Ethiopian cultivars at low $\mathrm{N}$, Dut $\mathrm{LN}=$ Dutch cultivars at low $\mathrm{N}$, Eth $\mathrm{HN}=$ Ethiopian cultivars at high $\mathrm{N}$, Dut $\mathrm{HN}=$ Dutch cultivars at high N. AUC = Area under the canopy curve in $\%$ thermal day $(\%$ td $)$, TYPP = Tuber yield plant ${ }^{-1}$ in $\mathrm{g}$.

\section{Interaction effect}

The combined analysis of variance over locations revealed that the cultivar effect was highly significant for all of the characters measured. Student's T-test was used to assess the effect of $\mathrm{N}$ level on various agronomic and physiological traits. The effect of $\mathrm{N}$ level was significant for most traits except stem number and some curve-derived thermal time parameters (Table 3). The interactions of $\mathrm{N}$ level $\mathrm{x}$ Cultivar, $\mathrm{N}$ level $\mathrm{x}$ Location and Location $\mathrm{x}$ Cultivar were highly significant for the majority of the evaluated traits. The three-way interaction ( $\mathrm{N}$ level $\mathrm{x}$ Location x Cultivars) was not significant for almost all characters except LCC and tuber dry weight (TDW). 
Table 3. Analysis of variance 18 traits of 97 potato cultivars in Debre-Tabor and Injibara at different $\mathrm{N}$ levels

Traits $\quad$ Significance of F value

\begin{tabular}{|c|c|c|c|c|c|c|c|}
\hline & $\begin{array}{l}\text { Genotype } \\
\text { (G) }\end{array}$ & $\begin{array}{l}\text { Location } \\
\text { (L) }\end{array}$ & $\begin{array}{l}\text { N level } \\
(\mathrm{N})\end{array}$ & G xL & $\mathrm{G} \times \mathrm{N}$ & $\mathrm{N} \times \mathrm{L}$ & GxNXL \\
\hline Degrees of Freedom & 96 & 1 & 1 & 96 & 96 & 1 & 96 \\
\hline DTE & $* *$ & $* *$ & $* *$ & $*$ & $*$ & ns & $\mathrm{Ns}$ \\
\hline $\mathrm{PH}$ & $* *$ & $*$ & $* *$ & $* *$ & $* *$ & $* *$ & Ns \\
\hline SNPP & $* *$ & $* *$ & ns & ns & ns & ns & Ns \\
\hline Vmax & $* *$ & $* *$ & $* *$ & * & $* *$ & $* *$ & Ns \\
\hline AUC & $* * *$ & ns & $* *$ & ns & $* * *$ & $* * *$ & Ns \\
\hline AP1 & $* * *$ & $* * *$ & $*$ & $* *$ & ns & $* * *$ & Ns \\
\hline Ap2 & $* * *$ & $* * *$ & $* *$ & $\mathrm{~ns}$ & ns & $* * *$ & Ns \\
\hline AP3 & $* * *$ & $* * *$ & $*$ & ns & ns & ns & Ns \\
\hline $\operatorname{tm} 1$ & $* * *$ & $* * *$ & ns & ns & ns & $* * *$ & Ns \\
\hline $\mathrm{t} 1$ & $* *$ & $* *$ & ns & ns & ns & ns & Ns \\
\hline $\mathrm{t} 2$ & $* *$ & $* * *$ & ns & ns & ns & $* * *$ & Ns \\
\hline $\mathrm{t} 2-\mathrm{t} 1$ & ns & $* * *$ & ns & ns & ns & $* * *$ & Ns \\
\hline te-t 2 & $* * *$ & $\mathrm{~ns}$ & $\mathrm{~ns}$ & $\mathrm{~ns}$ & $\mathrm{~ns}$ & $\mathrm{~ns}$ & Ns \\
\hline $\mathrm{Te}$ & $* * *$ & $* * *$ & ns & $* * *$ & ns & $* * *$ & Ns \\
\hline LCC & $* *$ & $* *$ & $* *$ & $* *$ & $* *$ & $* *$ & $* *$ \\
\hline DTM & $* *$ & $* *$ & $\mathrm{~ns}$ & $* *$ & $* *$ & $* *$ & Ns \\
\hline TNPP & $* *$ & $* *$ & $* *$ & $* *$ & $* *$ & $* *$ & $\mathrm{Ns}$ \\
\hline TYPP & $* *$ & $* *$ & $* *$ & $* *$ & $* *$ & $* *$ & Ns \\
\hline ATW & $* *$ & $\mathrm{~ns}$ & $* *$ & $* *$ & $*$ & $\mathrm{~ns}$ & Ns \\
\hline TDW & $* *$ & $* *$ & $* *$ & $* *$ & ns & $* *$ & $* *$ \\
\hline TDM\% & $* *$ & $* *$ & $* *$ & ns & ns & $* *$ & Ns \\
\hline SG & $* *$ & $* *$ & $* *$ & ns & ns & $* *$ & Ns \\
\hline NUE & $* *$ & $\mathrm{~ns}$ & $* *$ & $* *$ & ns & $*$ & Ns \\
\hline
\end{tabular}

$\mathrm{ns}=$ not significant; $*=$ significant at $\mathrm{P} \leq 0.05 ; * *=$ significant at $\mathrm{P} \leq 0.01 ; * * *=$ significant at $\mathrm{P} \leq 0.001 \mathrm{DTE}=\mathrm{Days}$ to emergence, $\mathrm{PH}=$ Plant height $(\mathrm{cm}), \mathrm{SNPP}=\mathrm{Stem}$ number plant $^{-1}, \mathrm{Vmax}=$ Maximum canopy cover in $\%$, tm $1=$ Inflection point in thermal day $(\mathrm{td}), \mathrm{t} 1=$ Canopy stabilized in $\mathrm{td}, \mathrm{t} 2=$ Onset of canopy senesced in td, $\mathrm{t} 2-\mathrm{t} 1=$ Duration for max canopy in td, te = Completely senesced canopy in td, $\mathrm{AUC}=$ Total area under the canopy in $\% \mathrm{td}, \mathrm{AP} 1=$ Area for growth phase one in $\% \mathrm{td}, \mathrm{AP} 2=\mathrm{Area}$ for growth phase two in $\%$ td, AP3 $=$ Area for growth phase three in \% td, LCC =Lower leaf chlorophyll content, UCC= Upper leaf chlorophyll content, DTM= Days to maturity Tuber

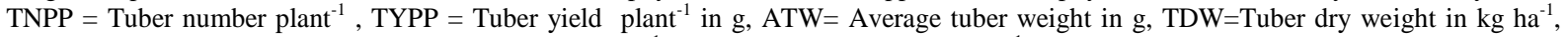
$\mathrm{TDM} \%=$ Tuber dry matter $(\%), \mathrm{SG}=$ Specific gravity $\mathrm{g} \mathrm{g}^{-1}$ Nitrogen use efficiency $\mathrm{kg} \mathrm{kg}^{-1}$, 


\section{Cluster analysis}

Cluster analysis was performed based on the mean data of 12 quantitative traits, in order to visualize genetic relationships of cultivar phenotypes at low and high $\mathrm{N}$ conditions across locations. Linked traits with double contribution (collinearity effect) were excluded from the cluster analysis. Means over the two locations were used as input for Unweighted Pair Group Method with Arithmetic Mean (UPGMA) hierarchical clustering and the 97 cultivars were clustered into 9 and 11 genetically distinct classes at low and high $\mathrm{N}$ at an average distance cut off value of 1.0 and 0.8 , respectively. However, not all cultivars ended up in the same clusters under low and high $\mathrm{N}$ levels. The group size varied from 1 to 84 cultivars at low $\mathrm{N}$ and from 1 to 63 cultivars at high N (Figure 4 and Figure 5).

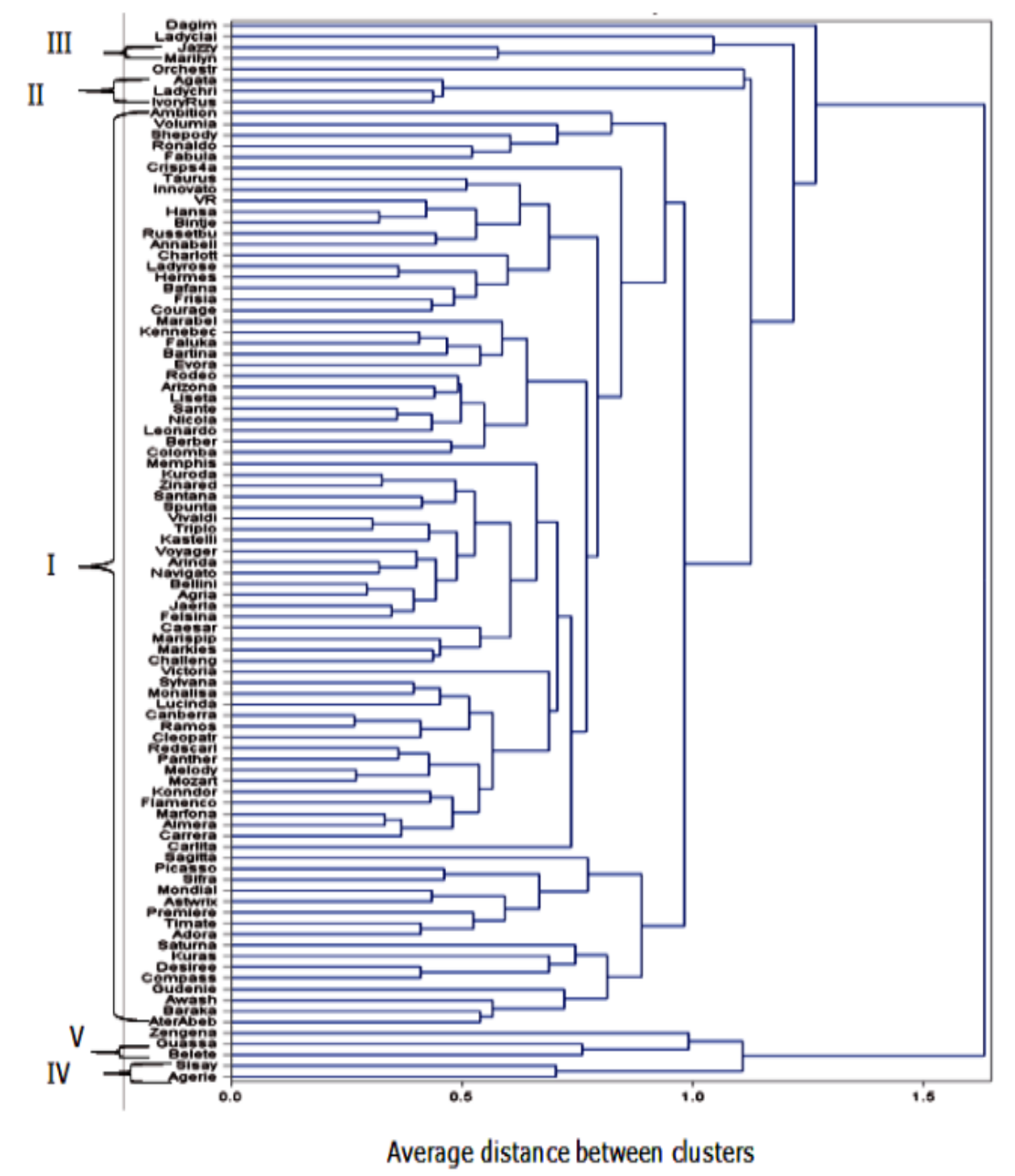

Figure 4. Average distance of 97 potato cultivar similarities based on 12 agronomic and physiological NUE related quantitative traits at low N using Unweighted Pair Group Method with Arithmetic Mean (UPGMA) hierarchical clustering. Traits: plant height, stem number, upper leaf chlorophyll content, canopy stabilized, completely senesced canopy,area under the canopy curve, tuber yield plant ${ }^{-1}$, tuber number plant ${ }^{-1}$, average tuber weight, tuber dry matter\%, and NUE 
Cluster I, II, III, IV and V consisted of more than one cultivar, while Zengena, Lady Claire, Orchestra and Dagim were cultivars that existed as singletons at low N. At high N, cluster I to cluster VII, were clusters that contained more than one cultivar, whereas Berber, Lady Claire, Fabula and Agerie were cultivars that existed as singletons. Most of the cultivars grouped in a single cluster (cluster I) at both $\mathrm{N}$ levels; $87 \%$ at low $\mathrm{N}$ and $65 \%$ at high $\mathrm{N}$. The Ethiopian cultivars Ater-Ababa, Awash and Gudenie were included in the largest cluster (cluster I) at low N level, the rest were Dutch cultivars. Most Dutch cultivars were clustered in cluster I, while the Ethiopian cultivars were distinctly grouped in cluster IV and $\mathrm{V}$ at low $\mathrm{N}$ and in cluster IV, VI and VII at high N levels, suggesting the presence of significant genetic distance between the Dutch and the Ethiopian potato cultivars.

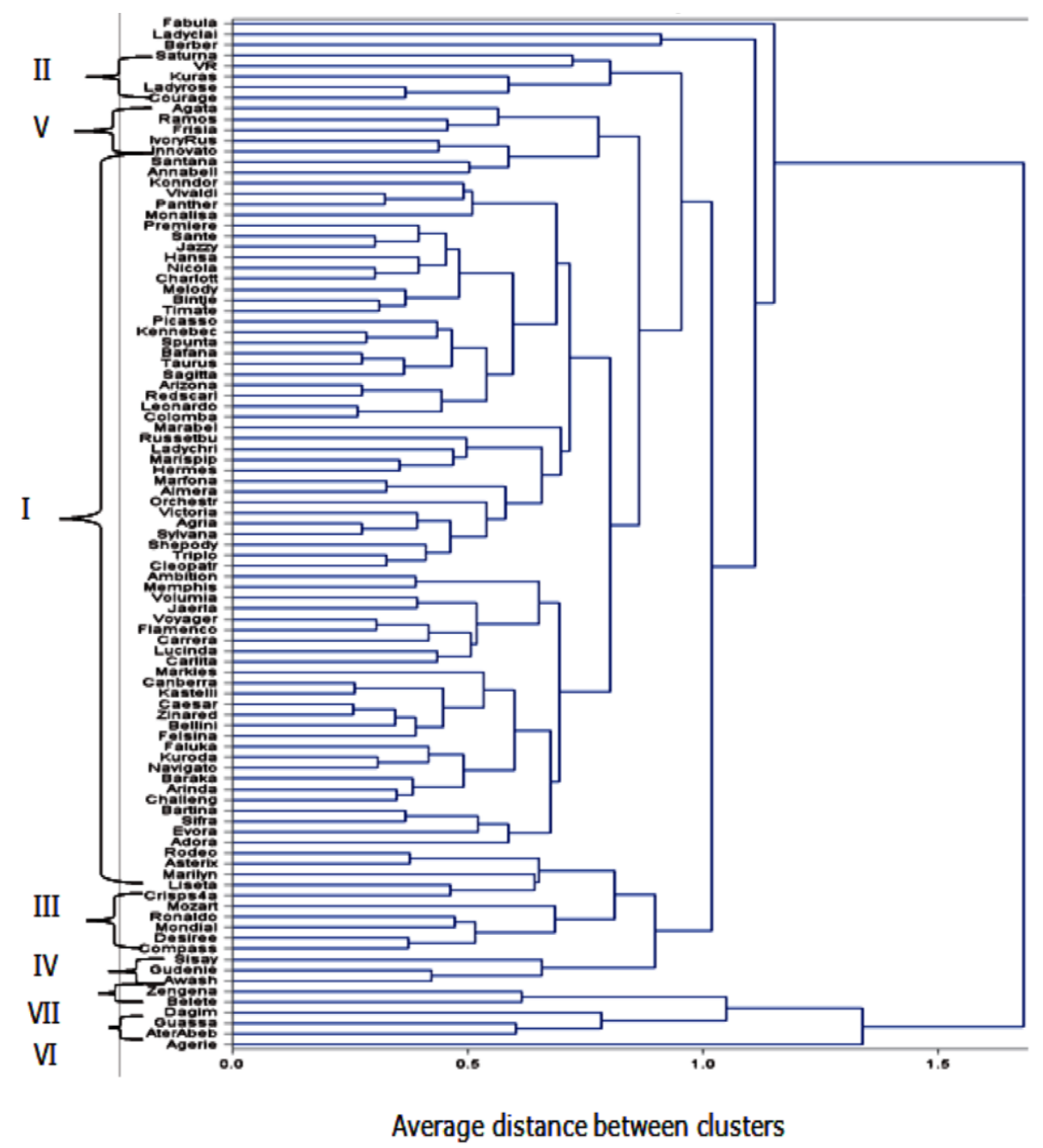

Figure 5. Average distance of 97 potato cultivar similarities based on 12 agronomic and physiological NUE related quantitative traits at high N using Unweighted Pair Group Method with Arithmetic Mean (UPGMA) hierarchical clustering. Traits: plant height, stem number, upper leaf chlorophyll content, canopy stabilized, completely senesced canopy,area under the canopy curve, tuber yield plant ${ }^{-1}$, tuber number plant ${ }^{-1}$, average tuber weight, tuber dry matter\%, NUE 
With the exception of the genetic distance between cluster I and II at low $\mathrm{N}$ and between cluster I and cluster III and V at high $\mathrm{N}$, the average inter-cluster squared distances $\left(\mathrm{D}^{2}\right)$ between clusters were significant at $(\mathrm{P} \leq 0.05$ and $\mathrm{P} \leq 0.01)$ at both $\mathrm{N}$ levels (Supplementary Table 2 and 3). There was a significant $(\mathrm{P} \leq 0.01) \mathrm{D}^{2}$ difference between the largest cluster (cluster I) and cluster III, IV and V at low N. The $\mathrm{D}^{2}$ between cluster I and cluster IV, VI, and VII was highly significant $(\mathrm{P} \leq 0.01)$ at high $\mathrm{N}$. DTM, PH, AUC, TYPP and NUE were the traits that contributed most to the difference between the Dutch and the Ethiopian set of cultivars at both $\mathrm{N}$ levels (Supplementary Table 4 and 5). Significant $(\mathrm{P} \leq 0.01) \mathrm{D}^{2}$ was observed between singleton cultivars and clusters that consisted more than one cultivar at both N levels: Zengena, Lady Claire, Orchestra and Dagim at low N, and Berber, Lady Claire, Fabula, and Agerie at high N (Supplementary Table 2 and 3). The lowest inter-cluster distance was recorded between cluster I and II at low N and between cluster I and III at high $\mathrm{N}$, indicating the relatively high relatedness of the cultivars included in the two large clusters at both $\mathrm{N}$ levels. The highest inter-cluster genetic distance was observed between two singleton cultivars, Zengena and Orchestra, at low N, and between cluster V and a single cultivar, Agerie at high N. Trait means of NUE and AUC under low N, and DTM and TNPP under high $\mathrm{N}$ condition were the main cause for the significant genetic distance difference between Zengena and Orchestra, and between cluster V and Agerie. Agerie, an Ethiopian traditional cultivar, was distinct mainly for its high number of tubers and late maturing characteristics.

\section{Estimates of genetic parameters}

Studies on genetic parameters and trait associations provide information about the expected response of different traits to selection and help in developing optimal breeding strategies (Gopal, 1999). We classified the observed variation in the potato cultivars into heritable and non-heritable components, and values for broad sense heritability $\left(\mathrm{H}^{2}\right)$, coefficient of phenotypic variation (PCV) and genotypic variation (GCV), and genetic advance as percent of mean (GA\%) obtained under low and high N level are presented in Supplementry tables 6 and 7. With the exception of Vmax, TDM\% at low N and NUE at both N levels in Injibara, estimates of $\mathrm{H}^{2}$ were high for the traits at both $\mathrm{N}$ levels and in both locations. Similarly, all traits had high GA\% except for UCC and DTM. $\mathrm{H}^{2}$ varied over treatments and locations between 0.33 and 0.95. NUE has high $\mathrm{H}^{2}$ values in Debre-Tabor at low and high $\mathrm{N}$ levels ( 0.80 and 0.72 respectively), but only 0.4 at both $\mathrm{N}$ levels in Injibara, indicating that the 
contribution of the environment to the total NUE variation was high in Injibara compared to Debre-Tabor.

The differences of $\mathrm{H}^{2}$ values of traits under high and low $\mathrm{N}$ conditions were small in most of the traits at both locations. This may suggest a weak interaction effect of $\mathrm{N}$ levels with the genotypes. However, with the exception of ATW, TNPP and UCC in Injibara, and TYPP and NUE in Debre-Tabor, all traits had higher $\mathrm{H}^{2}$ values at high $\mathrm{N}$ than at low $\mathrm{N}$ conditions, indicating that the environmental factors affect the measured traits more under $\mathrm{N}$ limited conditions compared to under high $\mathrm{N}$ conditions. The differences between PCV and GCV were minimal for all measured traits over treatments and locations, revealing that the contribution of genetic factors to the total phenotypic variation was large compared to the environmental factors, in line with the relatively high heritability estimates. For most traits considered in this study, a high value of genetic parameter estimates was observed at low and high $\mathrm{N}$ levels and across locations suggesting that the measured traits in our experimental setup can be used for genetic improvement through selection.

\section{Correlation and path analysis}

Information of mutual association between yield and yield component traits is important for effective utilization of genetic resources. Estimations of location-combined phenotypic correlation coefficients between traits under low $\mathrm{N}$ and high $\mathrm{N}$ is presented in Table 4. Low to high correlation coefficient values were found between the traits and NUE across locations. Strong phenotypic correlation coefficients were observed between NUE and all traits except ATW and UCC across N levels. Most of the traits showed higher phenotypic correlation coefficient values at high $\mathrm{N}$ level than at low $\mathrm{N}$ level. The correlation coefficients of traits between low and high $\mathrm{N}$ level were high except for UCC and te, which implies the effect of $\mathrm{N}$ levels was small on the association of traits and the trait may be in the same chromosomal region at both $\mathrm{N}$ levels (Table 4).

The correlations between traits presented in Table 4 do not indicate the cause and effect relationship, because different traits may contribute positively or negatively to the observed correlation coefficient between the two traits due to the physiological interrelationships among traits. Estimation of correlation components using path analysis (Figures 6 and 7) revealed that the largest direct contributions to the variation observed in NUE under low and high $\mathrm{N}$ condition were of TDM\% and TNPP. ATW also had a strong positive direct effect on NUE, even though the phenotypic correlation coefficient between the two traits was not significant. This week correlation between ATW and NUE resulted from the strong negative 
indirect effect of ATW via TNPP and TDM\% on NUE. Except in Debre-Tabor at high N level, TDM\% had the strongest direct influence on NUE at both $\mathrm{N}$ levels and in both locations. Some traits did not have a strong direct effect on NUE, however they had a strong indirect effect via the other traits (Figure 6 and 7). For instance, AUC had a negligible direct effect on NUE, while its high indirect effect via TDM\% and TNPP counterbalance the negligible direct effect on the observed variation of NUE. ATW and TNPP had a strong direct contribution for the variation observed on NUE, but their indirect effect via each other on NUE was negative. 


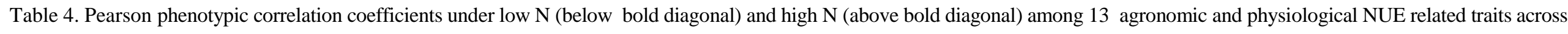
locations

\begin{tabular}{|c|c|c|c|c|c|c|c|c|c|c|c|c|c|}
\hline Low N & $\begin{array}{c}\text { High N } \\
\text { PH }\end{array}$ & UCC & $\mathrm{t} 1$ & $\mathrm{t} 2$ & te & Vmax & AUC & DTM & TNPP & ATW & TYPP & TDM\% & NUE \\
\hline $\mathrm{PH}$ & $0.95 * * *$ & -0.02 & $0.29 * *$ & $0.42 * * *$ & $0.61 * * *$ & $0.76 * * *$ & $0.78 * * *$ & $0.69 * * *$ & $0.37 * * *$ & $0.21 *$ & $0.65^{* * *}$ & $0.27 * *$ & $0.69 * * *$ \\
\hline UCC & -0.10 & 0.19 & -0.11 & 0.07 & 0.00 & -0.17 & -0.13 & 0.01 & -0.16 & $0.20 *$ & -0.01 & -0.01 & -0.02 \\
\hline $\mathrm{t} 1$ & $0.43 * * *$ & -0.11 & $0.55^{* * * *}$ & $0.46 * * *$ & $0.48 * * *$ & $0.32 * *$ & $0.35 * *$ & $0.34 * *$ & $0.22 *$ & 0.02 & $0.31 * *$ & 0.04 & $0.28 * *$ \\
\hline $\mathrm{t} 2$ & $0.33 * *$ & -0.12 & $0.42 * * *$ & $0.46^{* * *}$ & $0.42 * * *$ & $0.37 * * *$ & $0.52 * * *$ & $0.39 * * *$ & 0.17 & 0.04 & $0.44 * * *$ & $0.21 *$ & $0.48 * * *$ \\
\hline te & $0.58 * * *$ & -0.09 & $0.60 * * *$ & $0.41 * * *$ & $0.24 *$ & $0.65 * * *$ & $0.79 * * *$ & $0.84 * * *$ & $0.29 * *$ & $0.31 * *$ & $0.64 * * *$ & $0.25^{*}$ & $0.68 * * *$ \\
\hline$V \max$ & $0.44 * * *$ & $-0.25^{*}$ & $0.49 * * *$ & $0.35 * *$ & $0.50 * * *$ & $0.62 * * *$ & $0.95 * * *$ & $0.66 * * *$ & $0.59 * * *$ & 0.09 & $0.73 * * *$ & $0.25^{*}$ & $0.76 * * *$ \\
\hline AUC & $0.52 * * *$ & $-0.20^{*}$ & $0.56 * * *$ & $0.51 * * *$ & $0.66 * * *$ & $0.95 * * *$ & $0.71 * * *$ & $0.75 * * *$ & $0.55 * * *$ & 0.13 & $0.78 * * *$ & $0.30 * *$ & $0.83 * * *$ \\
\hline DTM & $0.64 * * *$ & -0.09 & $0.42 * * *$ & $0.27 * *$ & $0.76 * * *$ & $0.35 * *$ & $0.46^{* * *}$ & $0.63 * * *$ & $0.32 * *$ & $0.32 * *$ & $0.71 * * *$ & $0.29 * *$ & $0.75 * * *$ \\
\hline TNPP & $0.37 * * *$ & $-0.24 *$ & $0.28 * *$ & $0.35 * *$ & $0.39 * * *$ & $0.40 * * *$ & $0.47 * * *$ & $0.36^{* * *}$ & $0.81 * * *$ & $-0.58 * * *$ & $0.41 * * *$ & $0.40 * * *$ & $0.58 * * *$ \\
\hline ATW & $0.21 *$ & $0.23 *$ & 0.09 & -0.08 & 0.10 & -0.11 & -0.09 & 0.05 & $-0.58 * * *$ & $0.79 * * *$ & $0.40 * * *$ & $-0.49 * * *$ & 0.08 \\
\hline TYPP & $0.65 * * *$ & -0.03 & $0.53 * * *$ & $0.40 * * *$ & $0.59 * * *$ & $0.41 * * *$ & $0.51 * * *$ & $0.47 * * *$ & $0.41 * * *$ & $0.40 * * *$ & $0.62 * * *$ & -0.10 & $0.80 * * *$ \\
\hline TDM\% & $0.23 *$ & -0.10 & 0.07 & 0.04 & $0.25^{*}$ & $0.32 * *$ & $0.33 * *$ & $0.23 *$ & $0.35 * *$ & $-0.39 * * *$ & -0.03 & $0.76 * * *$ & $0.44 * * *$ \\
\hline NUE & $0.42 * * *$ & -0.06 & $0.50 * * *$ & $0.33 * *$ & $0.52 * * *$ & $0.58 * * *$ & $0.60 * * *$ & $0.47 * * *$ & $0.28 * *$ & 0.09 & $0.44 * * *$ & $0.39 * * *$ & $0.78 * * *$ \\
\hline
\end{tabular}

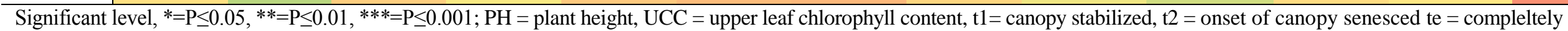

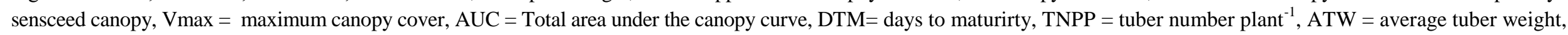
TYPP $=$ tuber yield plant $^{-1}$, TDM $\%=$ tuber dry matter $\%$, NUE $=$ nitrogen use effeciency 


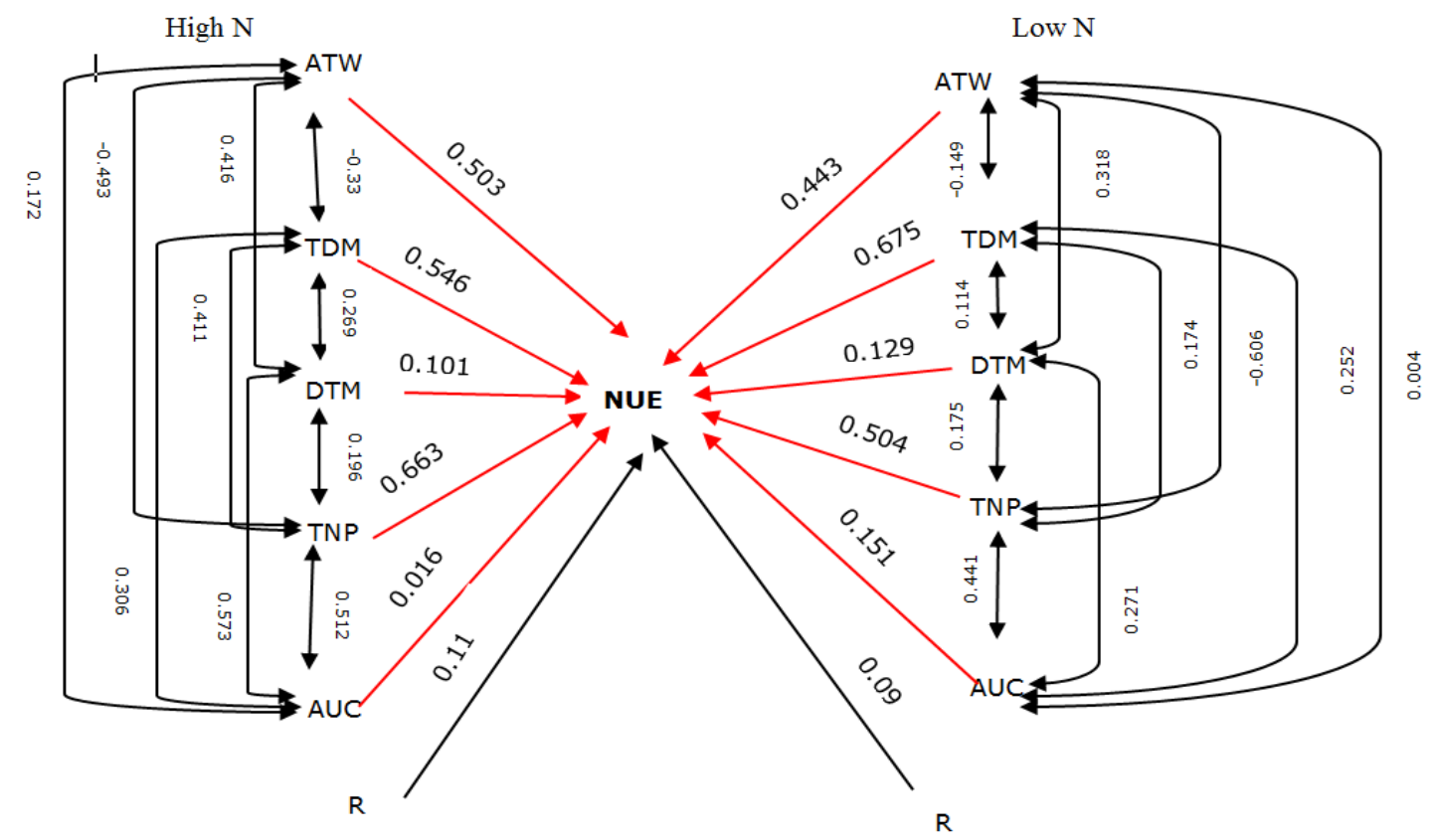

Figure 6. Diagram showing correlations and path coefficients of 5 factors influencing NUE under high and low N condition in Debre-Tabor. Double arrow lines indicate mutual association as measured by correlation coefficients and the red color single arrowed lines denote direct influence as measured by path coefficients. Negligible correlation and path coefficients were omitted. NUE: Nitrogen use efficiency, ATW: average tuber weight, TDM: tuber dry matter in \%, DTM: days to maturity, TNPP: tuber number plant ${ }^{-1}$, AUC: area under the canopy curve

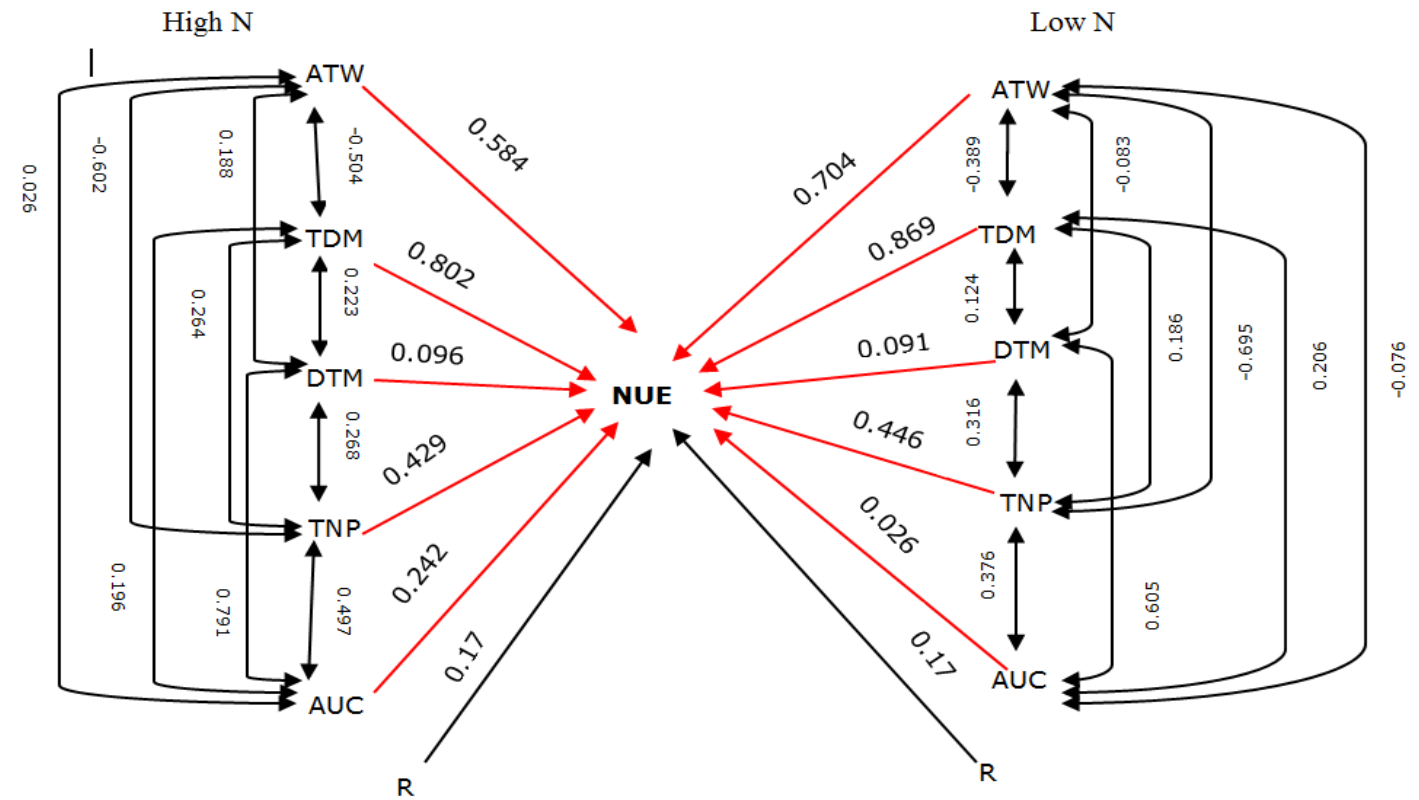

Figure 7. Diagram showing correlations and path coefficients of 5 factors influencing NUE under high and low N condition in Injibara. Double arrow lines indicate mutual association as measured by correlation coefficients and the red color single arrowed lines denote direct influence as measured by path coefficients. Negligible correlation and path coefficients are omitted. NUE: Nitrogen use efficiency, ATW: average tuber weight, TDM: tuber dry matter in \%, DTM: days to maturity, TNP: tuber number plant ${ }^{-1}$, AUC: area under the canopy curve 


\section{Discussion}

Genetic variation is a precondition for breeding programs aimed at potato improvement. The results of analysis of variance in the present study showed that the effect of genotype was significant for almost all of the measured traits including NUE, which implies the tested cultivar set had significant variation that may be used for breeding to improve NUE in potato. For several traits the Ethiopian cultivars behaved distinctly different from the Dutch cultivars. Dutch cultivars showed rapid initial canopy development and they matured early compared to the Ethiopian cultivars at both $\mathrm{N}$ levels and locations. This may be associated with limited adaptation to the experimental conditions, since the Ethiopian cultivars were selected under Ethiopian conditions while the Dutch cultivars were selected for Western-European, long day conditions. Haverkort (1990) suggested that potato varieties adapted to long day conditions may mature earlier and senesce between 60-70 days after emergence when grown around the equator. This physiological change is likely related to environmental factors like photoperiod and temperature. According to Ewing and Struik (1992), photoperiod and temperature are the major environmental factors that influence the growth of potato. Reduction in vegetative growth, early tuberization and senescence are the response of potato under short day condition (Maris 1964; Haverkort 1990; Van Dam et al.,1996).

The late maturity group of cultivars showed higher values for AUC and TYPP in Debre-Tabor compared to early and intermediate groups, however in Injibara the variation among maturity groups was not visible. In fact, in Injibara the late maturity group had higher AUC values compared to the early and intermediate group but there was no visible difference in tuber yield, which may indicate that radiation use efficiency (RUE) of these late cultivars was lower compared to the early and intermediate varieties. The reason for the lower RUE of the later maturing cultivars especially at Injibara is not known, however the soil of Injibara is very strongly acidic with $\mathrm{pH}$ value (4.8) and the average night temperature is low $\left(8^{0} \mathrm{C}\right)$, compared to Debre-Tabor with pH value (5.2) and higher night temperature. Okazawa (1967) reported that low $\mathrm{pH}$ inhibited lateral shoot growth and retard tuberization. According to the author, in potato plants, enzymes like amylase and phosphorylase which are responsible for the carbohydrate metabolism would play an important role in tuber formation, and the optimum $\mathrm{pH}$ value of these enzymes ranges between 6.0 and 7.0. Consequently, it seems reasonable to assume that the low soil $\mathrm{pH}$ value may have affected the tuber yield of potato cultivars in Injibara. 
Except Vmax and AUC, the effect of $\mathrm{N}$ level on canopy parameters was not significant. However, regardless of the effect of $\mathrm{N}$ levels, there was a difference in growth phase durations. Duration to reach maximum canopy cover (t1) was relatively longer than duration for maintenance of maximum canopy ( $\mathrm{t} 2-\mathrm{t} 1)$ at both $\mathrm{N}$ levels, resulting in an area under the curve for growth phase one (AP1) was greater than the area for the maximum canopy maintenance phase (AP2). Conversely, Ospina et al. (2014) using genotypes with similar genetic background but under European environmental conditions reported that high $\mathrm{N}$ enhanced $\mathrm{t} 2-\mathrm{t} 1$ and the duration for maintenance of maximum canopy ( $\mathrm{t} 2-\mathrm{t} 1)$ was higher than duration to reach maximum growth (t1). AP1 thus decreased due to short duration of $\mathrm{t} 1$, and AP2 increased due to long duration of t2-t1. The contrasting results for growth phases duration ( $\mathrm{t} 1$ and $\mathrm{t} 2-\mathrm{t} 1$ ) between the two environments (tropical and temperate), may be related with the reduction in time to mature, as our genotypes matured on average in less than 70 days under Ethiopian conditions whereas it took more than 100 days under European conditions.

The tuber yield reduction due to $\mathrm{N}$ shortage was substantial and significant in both locations. Of the two tuber yield components, tuber number had a larger effect on total tuber yield than average tuber weight especially in Injibara, which indicates that tuber bulking may be less affected than tuberization by limiting $\mathrm{N}$ conditions. Previous studies indicated that limited $\mathrm{N}$ availability prior to tuberization leads to low yield due to poor tuberization (Roberts et al., 1982; Dubetz and Bole, 1975). The reduction in tuber number with low N supply in Injibara may also be related to the soil chemical properties, as low $\mathrm{pH}$ affects tuber formation of potato (Okazawa, 1967). This may also affect the availability of important macro and micro nutrients and the physiological activity of the crop (Robson and Abbott, 1989). The high values for TDM\% in Injibara may be related to the lower temperature at this location, because when the temperature is high there will be high competition between vegetative growth and tuber bulking, and there may be high vegetative growth in the expense of tuber bulking. According to Winkler, (1971) and Ewing (1981), at low temperatures there is high assimilate accumulation and lower transpiration, and no considerable vegetative growth at the expense of tuber bulking.

In potato, genetic variation of NUE has been largely explained by maturity type (TiemensHulscher et al., 2012; Ospina et al., 2014). In our study, high NUE values were recorded at low $\mathrm{N}$ with late maturing potato cultivars. Similar results were reported by others (Ospina et 
al., 2014; Khan et al., 2013; Zebarth et al., 2004b; Zvomuya and Rosen, 2002). The long vegetative period may be the cause for the strong relationship between NUE and late maturity as late maturing cultivars have more time to accumulate assimilates compared to early cultivars. In this study, irrespective of the environment and $\mathrm{N}$ levels some of the late maturing potato cultivars like Kuras, Asterix from the Dutch and most Ethiopian varieties showed a relatively better NUE performance than the other Dutch cultivars, indicating the persistent inherent potential of the cultivars for NUE at both low and high $\mathrm{N}$ conditions.

In the cluster analysis, most of the cultivars grouped in a single cluster (cluster I) at both $\mathrm{N}$ levels. Eighty-seven percent at low $\mathrm{N}$ and sixtyfive percent at high $\mathrm{N}$ were grouped in cluster $\mathrm{I}$, and four cultivars at each $\mathrm{N}$ level were grouped separately, which implies that the diversity of the population or the compositional similarities between genotypes in the population was not proportionally distributed. Broad genetic distance was observed between Zengena and Orchestra at low $\mathrm{N}$ and between cluster V and Agerie at high N, however the most contrasting cluster means with significant inter-cluster genetic distance was shown between cluster II and Zengena at low $\mathrm{N}$, and cluster VII and Berber at high $\mathrm{N}$ for our targeted traits days to maturity, NUE, TYPP and AUC. This indicates that crosses of parents from these paired clusters/cultivars at the respective $\mathrm{N}$ levels will be expected to give suitable segregates for those specified traits.

Coefficients of variation (PCV and GCV) measure the magnitude of variation present in a population. The results in this study revealed that estimates of PCV were quite close to the estimates GCV for all measured traits over treatments at each location, indicating negligible environmental effect on the variance of traits. Similarly, Gopal (1999) using clones from Indian potato breeding programs and in Indian autumn and spring production season and Baye et al. (2005) using CIP-sourced Ethiopian breeding clones under rain fed production season reported high PCV and GCV values for plant height, tuber yield, average tuber weight and tuber number, which verifies that the genetic effect is consistently contributing highly to the measured variation of these traits in different testing materials and environments. Implicitly the contribution of the environment for the total variation of the traits is small compared to the contribution of the genetic component, indicating the traits are well heritable and suitable for selection. The contribution of environmental variance to phenotypic variance at low $\mathrm{N}$ was a bit greater than at high $\mathrm{N}$. Possibly high $\mathrm{N}$ input can mask soil heterogeneity more than low $\mathrm{N}$ input, and as a result environmental variance was higher at low $\mathrm{N}$ than at high N supply (Bertin and Gallais, 2001; Presterl et al.,2003). 
With the exception of Vmax and TDM\% at low N and NUE at both N levels which exhibited medium $\mathrm{H}^{2}$ in Injibara, the $\mathrm{H}^{2}$ estimates were high for all traits at both $\mathrm{N}$ levels and in both locations, which indicates the suitability of these characters for genetic improvement through selection. Similar results were reported for several other studies (Regassa and Barasavaj, 2005; Baye et al., 2005; Chaudhary, 1985; Desai and Jaiminis, 1997) and this similarity of heritability estimates in different testing environments and materials for plant height, tuber yield, average tuber weight and tuber number suggests that these genetic factors have a robust contribution to the total phenotypic variation of the traits.

All traits showed high GA\% accompanied with high $\mathrm{H}^{2}$ (except for chlorophyll content and days to maturity, which had low to medium genetic advance values across $\mathrm{N}$ levels and locations) indicating most likely the $\mathrm{H}^{2}$ is due to additive gene effects and early generation selection may be effective for these traits. As Johnson et al. (1955) stated, the estimates of GA\% are more important as a means of selection when considered jointly with the estimates of $\mathrm{H}^{2}$. High and low values of GA\% are indicative for additive gene action and non-additive gene action (Singh and Narayanan, 1993). The $\mathrm{H}^{2}$ estimates for the prediction of selection will therefore be reliable if it is accompanied by high GA\% estimates. Neele et al. (1991) also reported that an additive gene effect was more important in determining the inheritance of tuber yield and yield related traits. Conversely, Gopal (1998) reported that non-additive gene effect was more important than additive gene effect in determining yields, TNPP and ATW. In our study, ATW, TNPP, and AUC showed consistently high $\mathrm{H}^{2}$ and GA\% values across treatments and locations. Thus, based on our results can be based on these traits and their phenotypic expression would be a good indicator of their genotypic potential.

The path analysis showed that the direct effect of TDM\% and TNPP, and their indirect effects via DTM and AUC were substantial, indicating that direct selection with these traits can give satisfactory gain in NUE. However, although DTM and AUC had a strong positive correlation with NUE and considerable indirect effects via TDM\%, and TNPP, their direct contribution for NUE variation were minor. In these situations the best strategy, according to Neder et al. (2013), should be the simultaneous selection of traits, targeting those with significant indirect effects. The over-location residual effect $(\mathrm{R})$ of $\mathrm{N}$ levels ranged from 0.09 to 0.17 , indicating that more than $83 \%$ of the variability in NUE was contributed by the nine traits studied in the path analysis. This residual effect towards NUE in the present study may be due to various reasons such as other traits which are not included in this study, environmental factors and 
sampling errors (Sengupta and Karatia, 1971). Generally, from the present investigation it can be suggested that the potato cultivars evaluated in this study can be exploited for NUE improvement through improving and pyramiding of component traits such as TNPP, AUC, DTM, TDM\% and ATW. However, to use the above proposed traits as indirect selection criteria for NUE improvement further multi-year and location trials are required. 


\section{Chapter 3}

\section{Identification of QTLs associated with nitrogen use efficiency and}

related traits in a diploid backcross potato population

Baye Berihun Getahun ${ }^{1,2}$, Richard GF Visser ${ }^{1,2}$, C. Gerard van der Linden ${ }^{1,2}$

${ }^{1}$ Graduate School of Experimental Plant Sciences, Wageningen University \& Research PO Box 386, 6700 AJ Wageningen, The Netherlands

${ }^{2}$ Plant Breeding, Wageningen University \& Research, PO Box 386, 6700 AJ Wageningen, The Netherlands 


\begin{abstract}
Developing $\mathrm{N}$ use efficient potato varieties requires exploring the genetic basis of nitrogen use efficiency (NUE) and associated agronomic and physiological traits. In order to identify QTLs for NUE and NUE-related traits, and to determine the relationships between the traits and QTLs in potato, a diploid potato mapping population $(\mathrm{CxE})$ was evaluated in the field in Ethiopia under low and high $\mathrm{N}$ fertilizer levels. QTL detection was performed using interval mapping and multiple QTL mapping (MQM). A total of 52 putative QTLs were identified for ten traits, of which 28 QTLs were detected under low $\mathrm{N}$ availability while the remaining 24 QTLs were detected under high N conditions. Several QTLs were location and N level specific, suggesting the presence of QTL x environment interaction. The significant positive phenotypic correlations between different traits and co-localization of QTLs at specific regions in the genome demonstrated genetic and functional relations between these traits. A region on linkage group V (21-38cM) accumulated the largest number of QTLs. This region coincides with the earliness locus encoded by the CDF1 gene, suggesting that earliness had a profound influence on NUE. A putative second QTL region on linkage group V located 20cM from the earliness locus $(38-56 \mathrm{cM})$ that may be separate from the earliness locus, and a region on linkage group IV $(60-72 \mathrm{cM})$ might be useful regions to focus on for NUE improvement in potato. To verify the stability of the identified QTLs and to use these for further investigation and detection of possible candidate genes, further multi-environment trials with larger population size may be required.
\end{abstract}

Key words: Potato, NUE, QTL, nitrogen 


\section{Introduction}

Crop productivity is greatly affected by nutrient availability and nutrient use efficiency. Nitrogen Use Efficiency (NUE) has become the second priority production constraint after drought in crop abiotic stress improvement programs (Hirel et al., 2011). Indeed, improving agronomic NUE is relevant for the majority of crops currently cultivated; Less than $50 \%$ of the applied nitrogen is typically used by most crops and a large amount of $\mathrm{N}$ fertilizer is required to reach maximum yield (Zhang et al., 2007). The $\mathrm{N}$ that is not utilized by the plant is lost due to nitrate leaching, denitrification and loss of ammonia to the atmosphere which has a harmful effect on the environment as well as on the economy (Glass, 2003).

$\mathrm{N}$ availability affects many developmental processes, depending on the plant species (Zheng, 2009). In potato, $\mathrm{N}$ availability affects rate of canopy development and leaf appearance, final leaf size, rate photosynthesis, onset of tuberization, final tuber yield and harvest index (Vos and Biemond, 1992, Ewing \& Struik, 1992; Vos, 1995; Vos \& MacKerron, 2000; Ospina et al., 2014). Deficiencies or variation in availability of nitrogen and other soluble nutrients cause poor vegetative growth and health, reduced pathogen and insect resistance, decreased tuber yields, and these affect tuber quality as well (Ojala et al., 1990, Olsen et al., 2003, Stark et al., 2004). In general, potato requires high amounts of $\mathrm{N}$ fertilizer to give maximum tuber yield, however the crop is relatively poor in agronomic NUE (yield produced per nitrogen applied). The high nitrogen requirement and low use efficiency is not only because of low $\mathrm{N}$ utilization by the plant, but also because of inefficient uptake due to its shallow inefficient root system (Munoz et al., 2005; Pack et al., 2006).

In potato, a wide range of variation in NUE has been reported in cultivated potato clonal selections, and accessions of wild potato species (Errebhi, Rosen, and Martin et al.,1998, Errebhi et al., 1999; Zebarth et al., 2004; Zvomuya et al., 2002; Sharifi et al., 2007), suggesting the possibility of improving NUE through breeding. Various traits related to NUE and contributing to NUE were used to increase the efficiency of the selection process and support the development of cultivars that give reasonable yield under low $\mathrm{N}$ availability (Errebhi, Rosen, and Martin et al., 1998). Among these traits, nitrogen uptake efficiency, yield and its components, Leaf Area Index (LAI) and period for maximum soil covering showed significant variation at low $\mathrm{N}$ conditions (Tiemens-Hulscher et al., 2012). However, the genetic basis of NUE is still poorly understood, and the complexity of many phenotypic traits involved in adaptation to stress conditions is likely to arise from a number of quantitative trait 
loci (QTL) (Bulmer et al., 1985; Falconer and Mackay, 1996). To dissect the complexity of such quantitative traits into component loci and identify the genetic factors that influence quantitative traits, QTL analysis/genetic mapping is a powerful tool (Doerge, 2002). For instance, a QTL approach offers an opportunity to dissect physiological and genetic components that affect the source-sink relationship under abiotic stress conditions (Pelleschi et al., 2006; Welcker et al., 2007; Miralles and Slafer, 2007), which is likely to be a major component for potato yield.

QTL analysis also provides opportunities for the analysis of the relationships between traits (Lebreton et al., 1995; Simko et al., 1997). Co-localization of QTLs for two traits that are phenotypically correlated is good evidence that the two traits are functionally and genetically linked (Quarrie, 1996; Thumma et al., 2001). Simko et al. (1997), used QTL analysis to evaluate the causal relationship between tuber dormancy and abscisic acid (ABA) content. In maize, the relationship between $\mathrm{ABA}$ as a major stress hormone with yield and other drought related traits was analysed using QTL approach (Lebreton et al., 1995; Quarrie, 1996). However, QTLs can be affected by environmental variation. Some QTLs exist consistently over environments (constitutive QTLs), while other QTL are identified only in specific environments, or modulate their effect with changing environmental conditions (adaptive QTLs) (Tuberosa et al., 2008). Studies of QTLs affecting traits related to NUE have been reported in maize (Agrama et al., 1999; Hirel et al., 2001), Arabidopsis (Loudet et al., 2003) and rice (Cho et al., 2007), and many of these QTLs were dependent on N levels.

In the last two decades many QTL analysis studies have been published on different traits of potato, such as flower colour, foliage maturity, tuber skin texture, dry matter content, specific gravity and yield (McCord et al., 2011), yield, agronomic and quality traits (Bradshaw et al., 2008), tuber yield and starch content (Schafer-Pregl et al., 1998), tuber dormancy (van den Berg et al., 1996), tuber shape (Van Eck et al., 1994), tuber skin colour (Gebhardt et al., 1989), tuber flesh colour (Bonierbale et al., 1988) and drought related traits (Anithakumari et al., 2011, 2012; Khan et al., 2014) .The number of QTL studies for NUE are still very limited. Only recently QTLs affecting traits related to NUE under contrasting N regimes were reported in potato (Ospina, 2016). The aims of the present study were: (1) To determine the chromosomal location and genetic effect of QTLs for NUE and traits associated with NUE in potato under low and high $\mathrm{N}$ conditions in Ethiopia, and (2) Deliver basic genetic and 
physiological information of NUE and related traits for future candidate gene identification and marker assisted selection studies.

\section{Materials and Methods}

\section{Plant materials}

One hundred individuals of a diploid backcross population (CxE) including the parents were used in this study. The population was obtained from the original cross between the female parent, C (USW5337.3) (Hanneman and Peloquin, 1967) and the male parent, E (77.2102.37) (Jacobsen, 1980). Clone C is a hybrid of S. phureja (PI225696.1) and S. tuberosum dihaploid USW42. Clone E is the result of a cross between clone C and the S. vernei-S. tuberosum backcross clone VH34211 (Jacobsen, 1980). Absence of dormancy, early maturity and short day tuberization are some of the characteristics of $S$. phureja. On the contrary, Solanum tuberosum is characterized by long dormancy, long day tuberization and variable maturity (Hawkes 1990; Ewing and Struik 1992).

\section{Field studies}

The field studies were conducted in Ethiopia at Koga from January to May 2014 and at Injibara and Debre-Tabor from July to October 2014 under irrigation and rainfed conditions, respectively. In Ethiopia, the rainfed production season is from June to October, and is fully dependent on rain water. The irrigation production season is from Nov-April and is fully dependent on irrigation water from rivers and streams. The experiment was laid out in a split plot arrangement with two replications, with the low and high $\mathrm{N}$ levels $\left(40 \mathrm{~kg} \mathrm{ha}^{-1}\right.$ and $120 \mathrm{~kg}$ $\mathrm{ha}^{-1}$ ) assigned as main plots and the genotypes as sub plots. Each replication consisted of 10 plants, planted at a recommended inter- and intra-row spacing of $0.75 \mathrm{~m}$ and $0.30 \mathrm{~m}$ respectively and each genotype replication was bordered by a plant from reference cultivar. Soil available nutrients and externally applied urea, Di-ammonium phosphate (DAP) and trisuperphosphate (TSP) were used as source of $\mathrm{N}$ and P. Composite soil samples were collected at five different locations in the experimental field and at a soil depths of $0-20 \mathrm{~cm}$ and 20 $40 \mathrm{~cm}$ before planting to estimate the available residual nitrogen in the form of $\mathrm{NO}_{3}{ }^{-}$and $\mathrm{NH}_{4}{ }^{+}$ using a $\mathrm{KCl}$ extraction method. The whole $\mathrm{P}$ source was applied at planting while $\mathrm{N}$ application was split in two: a week after emergence and at early flowering. Pest and disease management, weeding and ridging and other cultivation practices were conducted as per recommendation and when required. 


\section{Phenotypic measurements}

The phenotypic measurements were carried out in similar manner at all experimental locations (Koga, Debre-Tabor and Injibara). Plant height (PH), Chlorophyll content (CC) at lower and upper part leaf using SPAD-502 chlorophyll meter (Minolta Co., Ltd. Japan) were measured when $50 \%$ of the genotypes were flowering. The readings for chlorophyll content were taken on the third or fourth leaf from the top of the plant for upper leaf chlorophyll content (UCC), and the second or the third leaf from the base of the plant for lower leaf chlorophyll content (LCC). Stem number plant ${ }^{-1}$ (SNPP) was counted before the plant foliage declined. Canopy cover dynamics or soil cover (SC) was assessed every five days starting from date of emergence to the declining phase of the crop growth using a $0.6 \mathrm{~m} \times 0.75 \mathrm{~m}$ frame with 100 grid squares, positioned over the same middle plants in a plot for each measurement, and the canopy measurements were carried out from date of emergence to the end of the declining phase of the crop growth. The beta thermal time for canopy cover assessment was calculated from the date of emergence for each experimental unit, using the sigmoid part of the beta function for determinate growth (Yin et al., 2003), and an estimated cardinal temperature (with $5.5^{\circ} \mathrm{C}$ as a base temperature, $23.37^{\circ} \mathrm{C}$ as optimum, and $34.58^{\circ} \mathrm{C}$ as ceiling temperature) that determines the vegetative growth of potato (Khan 2012, Khan et al., 2013). Hourly temperature data was collected from the nearest weather station for each location. The model for canopy development was fitted using the soil cover data, beta thermal time for each assessment, and the canopy cover measurements, and the following model parameters were estimated: the inflection point in the build-up phase of the growth curve (tm1), time at which canopy cover reaches its maximum (t1), the maximum canopy cover value with percentage of soil cover as unit (Vmax), time for onset of canopy decline (t2), time when canopy cover reaches zero (te), and area under the curve for the entire crop growth cycle ( AUC ) in \%.td, were estimated using the NOLIN procedure of SAS, SAS Institute Inc, 2004 (Khan et al., 2013). Days to maturity (DTM), determined as the number of the days from emergence to the day at which more than 90 percent of the plants in a plot attained physiological maturity $(90 \%$ of the haulm tissues brown) was assessed every day starting from the time that early varieties showed the first signs of leaf yellowing.

Tuber traits: tuber number plant ${ }^{-1}$ (TNPP), average tuber weight (ATW), tuber yield plant $^{-1}$ (TYPP), Specific gravity (SG), tuber dry matter percentage (TDM\%) and Nitrogen Use Efficiency (NUE; defined as dry tuber weight per unit $\mathrm{N}$ available ( $\mathrm{N}$ applied $+\mathrm{N}$ available in the soil) were measured and estimated at harvest. Specific gravity (SG) was determined using 
the tuber specific gravity procedure of weight in air and under water (Murphy and Goven, 1959). In evaluating the SG of each variety, healthy and marketable-sized grade (20 $\mathrm{mm}$ and above) tubers were selected randomly from each variety harvest. Then, tubers were cleaned, and weighed both in air and water following the procedure of Murphy and Goven (1959). Specific gravity values were computed using the following formula:

$$
S G=\frac{W_{1}}{W_{1}-W_{2}}
$$

where $\mathrm{SG}=$ specific gravity of the material, $\mathrm{W}_{1}=$ weight in air of the sample tuber, in $\mathrm{g}$ and $\mathrm{W}_{2}=$ Weight of the sample completely immersed in water, in g. Tuber dry matter content (TDM\%) normally is determined as a ratio of dry tuber weight to fresh weight expressed in percentage; we determined TDM\% indirectly from SG using empirical conversion factors following the equation of Kleinkopf et al., (1987): solid (Dry matter \%) = -214.9206+ (218.1852 x SG). Tuber dry weight (TDW) was estimated indirectly from specific gravity and tuber dry matter content in percent, using the following formula:

$T D W=\frac{T D M \% * T F W}{100}$

Where TDW $=$ Tuber dry weight in $\mathrm{g}, \mathrm{TDM} \%=$ Tuber dry matter $(\%)$, TFW $=$ Tuber fresh weight in g. Nitrogen use efficiency (NUE) is calculated as the yield per unit of $\mathrm{N}$ resource available to the plant (Moll et al., 1982). In this study, NUE was determined as the tuber dry matter production, or tuber dry weight $\mathrm{ha}^{-1}$, per unit of $\mathrm{N}$ supplied/ha $(\mathrm{N}$ in the soil + applied $\mathrm{N})$.

\section{Statistical analyses}

Analyses of variance (ANOVA) of the different traits, correlation and principal component analysis (PCA) were done with GenStat software $17^{\text {th }}$ edition. Restricted maximum likelihood (REML) variance component analysis, genotypic variance $\left(\sigma^{2} \mathrm{~g}\right)$, environmental variance $\left(\sigma^{2} \mathrm{e}\right)$ and broad sense heritability $\left(\mathrm{H}^{2}\right)$ were estimated using Breeding View, the IBP Breeding Management system (BMS) version 3.0.9 (https://www.integratedbreeding.net/breeding-

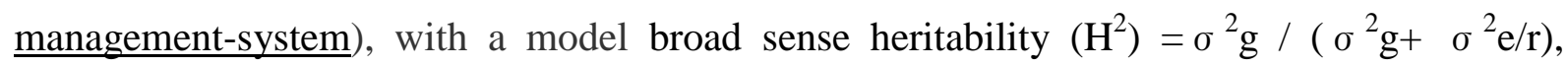
where $\left(\sigma^{2} \mathrm{~g}\right)$ is the genotypic variance, $\left(\sigma^{2} \mathrm{e}\right)$ is the environmental variance and $r$ is the 
number of replications. To generate phenotypic values for QTL mapping, the genotype was modelled as a fixed effect and all other effects were random, and the best linear unbiased estimates (BLUE) means were computed with BMS-breeding view software.

\section{Genetic map construction}

Details of the genetic map and markers employed like Simple Sequence Repeats (SSR), Amplified Fragment Length Polymorphism (AFLP), Cleavage Amplified Polymorphism (CAP) and Single Nucleotide Polymorphism (SNPs) can be found in Anithakumari et al., (2011). The integrated CE map constructed using JoinMap 4.0 (Kyazma, Van Ooijen 2006) was utilized for the QTL analysis.

\section{QTL analyses}

MapQTL6 (Kyazma, Van Ooijen 2009) was used for the QTL analysis. Each trait was analysed using interval mapping. For this analysis, a map with 12 linkage groups and 534 SNP markers with a total genetic map distance of $1326 \mathrm{cM}$ were employed, equivalent to an average distance between markers of $2.5 \mathrm{cM}$ assuming that these are equally distributed. Significance for QTL detection was determined by permutation tests (1000 permutations) and a genome wide scan was used as a QTL detection threshold at 5\% significant level. Subsequently, Multiple QTL Mapping (MQM) was performed with the markers nearest to the QTLs detected by interval mapping selected as cofactors.

\section{Results}

\section{Phenotypic variation, heritability and variance components}

The $\mathrm{C}$ x E diploid potato backcross population was grown at three different locations in two different production seasons (rainfed and irrigation) under low and high $\mathrm{N}$ fertilizer regimes to evaluate potato genotypes for NUE. NUE is defined in different ways, depending on the objective of the study and the crop under study (Good et al., 2004). In this study NUE is defined as the dry tuber yield per unit of nitrogen resource available to the plant. The combined analysis of variance over location showed that the genotypic variation was highly significant for all of the traits measured (supplementary Table 1), indicating that sufficient variation is present in the population for genetic analysis. In addition to the genotype, significant effects were observed for location, the interaction of location with $\mathrm{N}$ levels and genotype $\mathrm{x}$ location interaction for most agronomic and physiological traits. These results 
indicate that the genotype $\mathrm{x}$ location interaction often had a larger effect than the genotype $\mathrm{x}$ $\mathrm{N}$ level interaction, suggesting that genotype $\mathrm{x}$ location interaction contributed more to the total genotype $\mathrm{x}$ environment interaction.

Mean values of different yield and yield related traits of the parents $\mathrm{C}$ and $\mathrm{E}$, minimum and maximum performance of progeny and genetic variance components under low and high nitrogen condition across locations are presented in supplementary Table 2. The two parents (C and E) performed differently in tuber yield and foliage traits (canopy cover parameters) under both $\mathrm{N}$ conditions. Parameters describing different aspects of canopy growth and development were derived from the canopy cover dynamics of potato as quantified by the grid method, and modelled using beta thermal time as described in the material and methods section (Khan et al. 2013). Among the canopy cover parameters, the maximum value of the canopy cover (Vmax), and the total area under the canopy (AUC), which reflects the capacity of the crop to intercept solar radiation during the whole growing period, were the predominant traits that were different between the two parents. Parent $\mathrm{C}$ showed higher mean performance compared to parent $\mathrm{E}$ in tuber yield related traits as well as foliage traits under low and high $\mathrm{N}$ conditions in all locations except for maximum canopy cover (Vmax) and total area under the canopy cover (AUC) at Debre-Tabor, for which parent E performed better than parent C. The mean performance of the parents for most of the traits was a bit higher than the mean of the progeny.

The effect of $\mathrm{N}$ levels was significant (according to a student's T-test) for most agronomic and physiological NUE related traits considered in this study. Significant phenotypic variation $(\mathrm{P} \leq 0.001)$ was observed for $\mathrm{N}$ level in the $\mathrm{CE}$ population and between the two parents for most traits measured in this study except stem number plant ${ }^{-1}$ (SNPP), the inflection point in the build-up phase of the growth curve (tm1), time for onset of canopy decline (t2), and time when canopy is completely senesced (te). Low $\mathrm{N}$ application substantially affected agronomic and physiological traits of the parents, with stronger performance reductions for parent $\mathrm{E}$ than for parent $\mathrm{C}$. The overall differences for selected traits between parent $\mathrm{C}$ and parent $\mathrm{E}$, and the progeny are presented in Table 1. 
Table 1. Mean performance, Difference due to $\mathrm{N}$ level and difference of parent $\mathrm{C}$ and parent $\mathrm{E}$, and the progeny for some selected traits under low $\mathrm{N}(\mathrm{LN})$ and high $\mathrm{N}(\mathrm{HN})$ conditions

\begin{tabular}{|c|c|c|c|c|c|c|c|c|c|}
\hline \multirow[t]{3}{*}{ Traits } & \multicolumn{3}{|c|}{ Parent $\mathrm{C}$} & \multicolumn{3}{|c|}{ Parent E } & \multicolumn{3}{|c|}{ Progeny } \\
\hline & \multicolumn{2}{|c|}{ Mean } & \multirow{2}{*}{$\begin{array}{l}\text { Reduction } \\
(\%) \text { at } \\
\text { LN compared } \\
\text { to } \mathrm{HN}\end{array}$} & \multicolumn{2}{|c|}{ Mean } & \multirow{2}{*}{$\begin{array}{l}\text { Reduction (\%) at } \\
\text { LN compared to } \\
\text { HN }\end{array}$} & \multicolumn{2}{|c|}{ Mean } & \multirow{2}{*}{$\begin{array}{lr}\text { Reduction } & (\%) \\
\text { at } & \mathrm{LN} \\
\text { Compared } & \text { to } \\
\mathrm{HN} & \end{array}$} \\
\hline & $\mathrm{LN}$ & $\mathrm{HN}$ & & LN & $\mathrm{HN}$ & & $\mathrm{LN}$ & $\mathrm{HN}$ & \\
\hline $\mathrm{PH}$ & 31 & 37 & 17 & 36 & 43 & 16.7 & 28 & 35 & 20 \\
\hline LCC & 46 & 49 & 6 & 46 & 49 & 4.8 & 47 & 52 & 10 \\
\hline DTM & 88 & 85 & 2.5 & 87 & 85 & 1.7 & 86 & 84 & 2 \\
\hline TNPP & 8 & 13 & 36.9 & 6 & 9 & 38.7 & 7 & 10 & 30 \\
\hline ATW & 35.2 & 35.4 & 0.5 & 28.7 & 34 & 15.7 & 24 & 29 & 17 \\
\hline TYPP & 300 & 430 & 31.5 & 170 & 320 & 46.4 & 170 & 287 & 41 \\
\hline NUE & 57.8 & 26.6 & 117.3 & 37.76 & 22.85 & 65.3 & 34 & 18 & 89 \\
\hline Vmax & 40.5 & 50.9 & 20.5 & 31.75 & 46.43 & 31.6 & 27 & 40 & 33 \\
\hline AUC & 1138.23 & 1174 & 3.05 & 750.57 & 1139.4 & 34.13 & 649 & 953 & 32 \\
\hline
\end{tabular}

$\mathrm{PH}=$ Plant height in $\mathrm{cm}, \mathrm{LCC}=$ lower leaf chlorophyll content, DTM= Days to maturity, TNPP= Tuber number plant ${ }^{-1}, \mathrm{ATW}=$ Average tuber weight in $\mathrm{g}$, TYPP $=$ Tuber yield plant $^{-1}$ in $\mathrm{g}, \mathrm{NUE}=$ Nitrogen use efficiency $\left(\mathrm{kg} \mathrm{kg}^{-1}\right), \mathrm{Vmax}=$ maximum canopy cover in $\%, \mathrm{AUC}=$ Area under the canopy curve in $\%$ thermal day ( $\%$ td) 

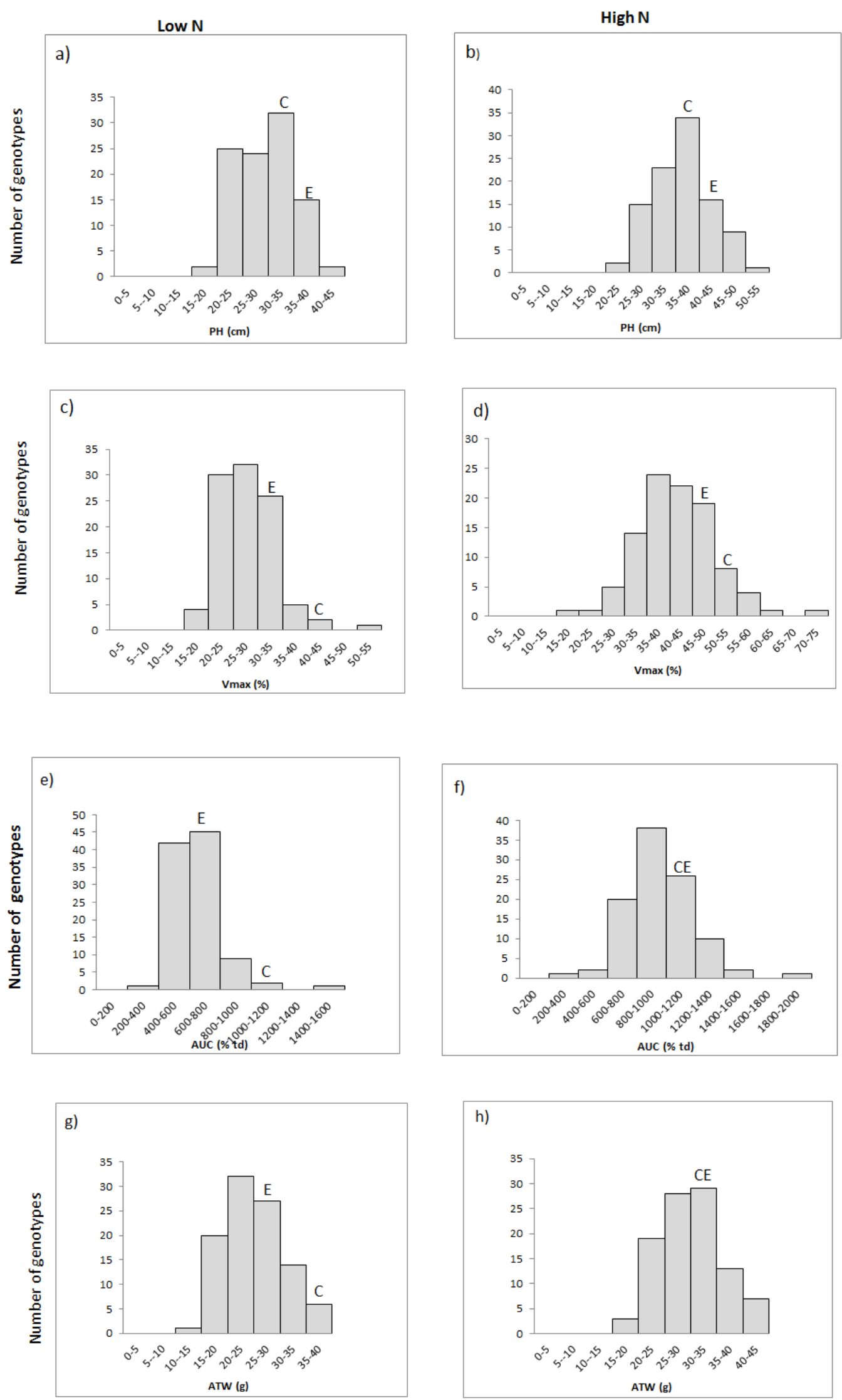

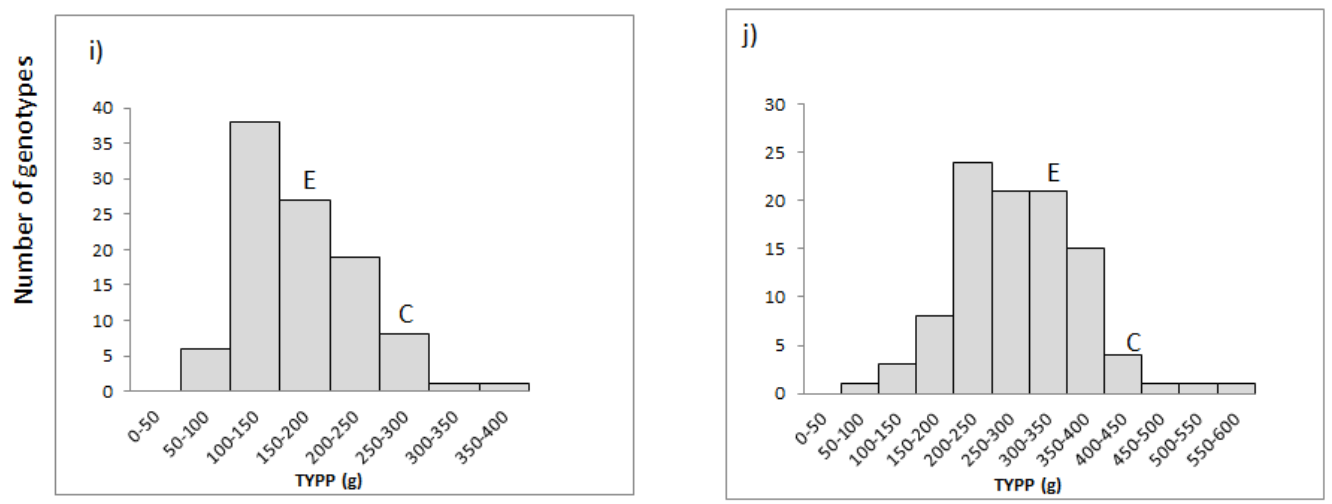

Figure 1. Effect of $\mathrm{N}$ levels on the phenotypic distributions of some selected traits for parent $\mathrm{C}, \mathrm{E}$ and the $\mathrm{C} \mathrm{xE}$ potato genotypes. $\mathrm{PH}=$ plant height in $\mathrm{cm}, \mathrm{Vmax}=$ maximum canopy cover in $\%, \mathrm{AUC}=$ area under the canopy curve in \%td, ATW = average tuber weight in g, TYPP = tuber yield plant ${ }^{-1}$. a) plant height at low N, b) plant height at high $\mathrm{N}, \mathrm{c}$ ) maximum canopy cover at low $\mathrm{N}, \mathrm{d}$ ) maximum canopy cover at high $\mathrm{N}$, e) area under the canopy curve at low $\mathrm{N}, \mathrm{f}$ ) area under the canopy curve at high $\mathrm{N}, \mathrm{g}$ ) average tuber weight at low $\mathrm{N}, \mathrm{h}$ ) average tuber weight at high $\mathrm{N}, \mathrm{i}$ ) tuber yield plant $^{-1}$ at low $\mathrm{N}, \mathrm{j}$ ) tuber yield plant $^{-1}$ at high $\mathrm{N}$

Significant differences $(\mathrm{P} \leq 0.05)$ in days to maturity (DTM) were found due to the effect of $\mathrm{N}$ levels, and of genotype. The genotypes matured on average between 75 and 95 days at both $\mathrm{N}$ levels. Based on the total number of days to reach maturity, genotypes were grouped as early (between 75 and 82 days), intermediate (between 83-89 days) and late maturing ones (90 days and above). Large differences were found between late and early maturing genotypes for Vmax, AUC, TYPP, and NUE at the same N level. Late maturing cultivars showed higher canopy cover compared to early ones under low $\mathrm{N}$ conditions (see example for some genotypes in Figure 2).

The heritability of the traits varied from 0 to 0.83 under low $\mathrm{N}$ and from 0.37 to 0.86 under high $\mathrm{N}$ conditions (supplementary Table 2). For most traits, the highest heritability was recorded at high $\mathrm{N}$ level compared to low $\mathrm{N}$. However, the heritability estimate difference between high and low $\mathrm{N}$ conditions was negligible. The highest heritability value difference (0.43) between high and low $\mathrm{N}$ conditions was observed for the trait AUC followed by LCC (0.40) at Debre-Tabor and Injibara respectively. Among the locations, Koga showed higher heritability estimates for most traits compared to Debre-Tabor and Injibara. Except tuber dry matter and chlorophyll content, for most traits the genotypic variance value is higher than that of environmental variance (supplementary Table 2), indicating that the contribution of the genetic factor to the total phenotypic variation was large compared to the environmental factor. 
a)

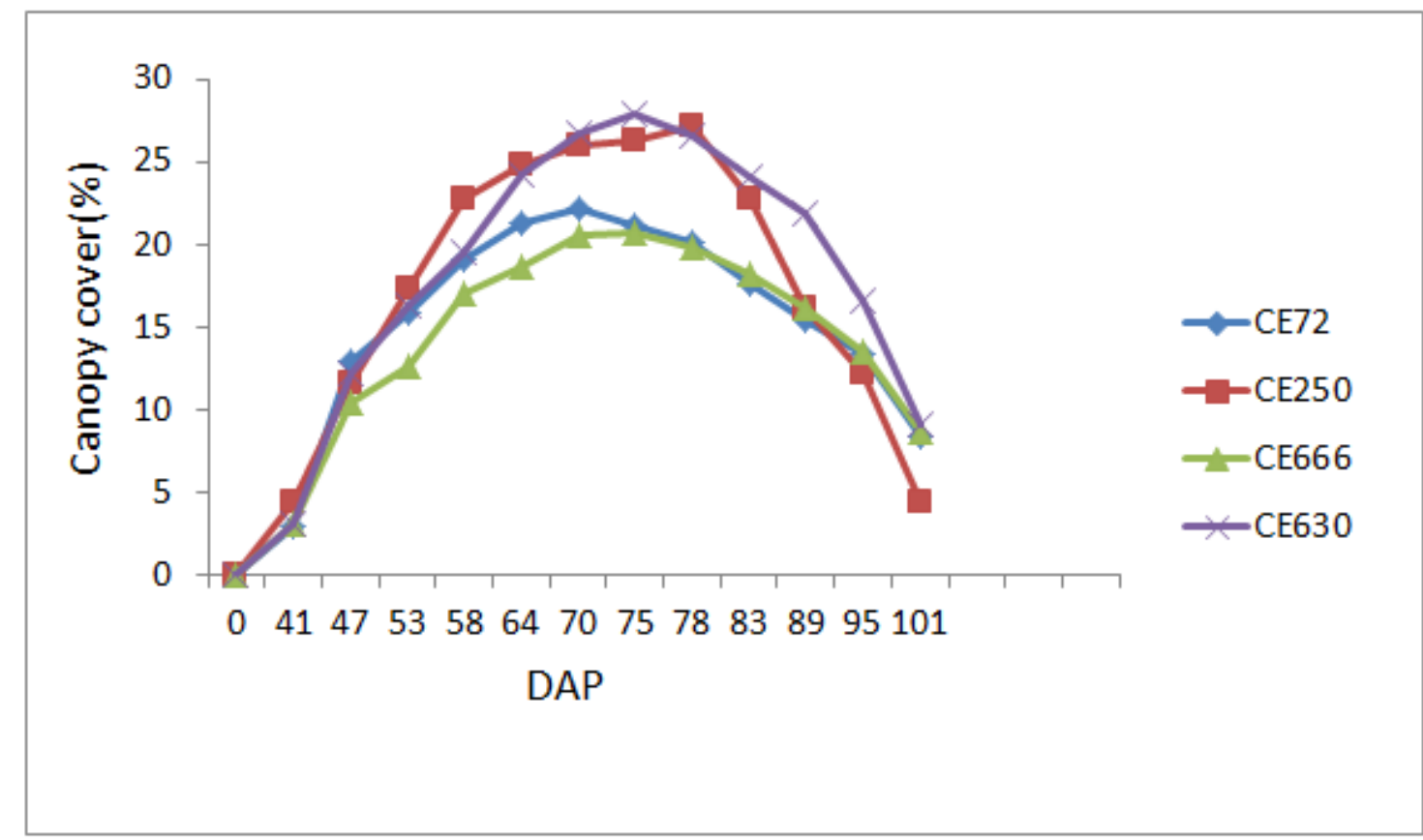

b)

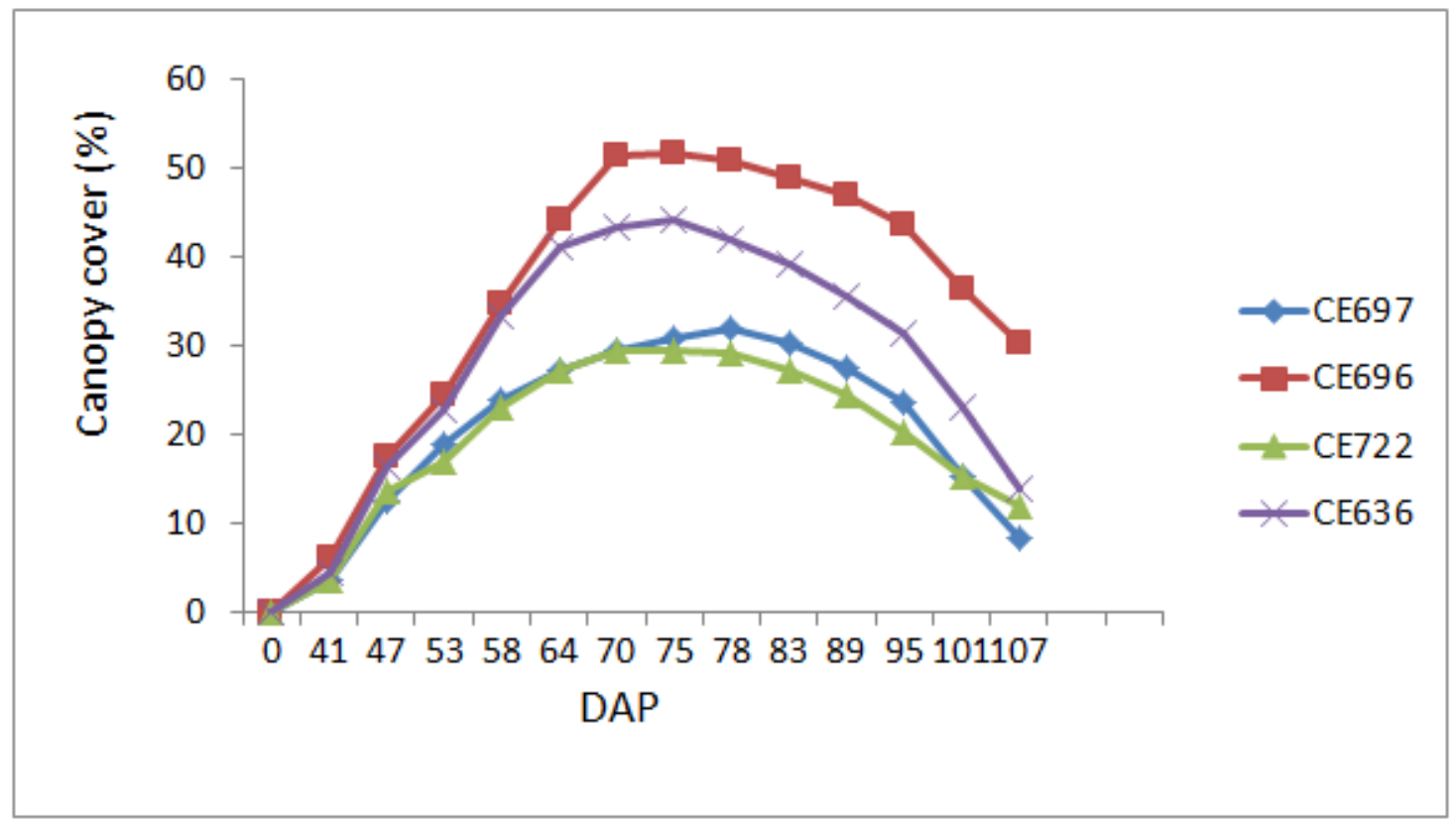

Figure 2. Difference in canopy development process of some CE genotypes selected randomly based on maturity group using raw canopy cover data and maturity data under low $\mathrm{N}$ condition, a) early maturing, b) late maturing. DAP = Days after planting 


\section{Correlation and Principal Component Analysis (PCA)}

The phenotypic correlation coefficients of traits under low and high $\mathrm{N}$ condition are presented in Table 2. The correlation between NUE and most agronomic and physiological traits was positive and significant both under low and high $\mathrm{N}$ conditions. However, depending on the traits, some differences in correlations were observed between low and high N levels. LCC and UCC are traits that correlate negatively with most traits at both N levels. The two tuber yield component traits (TNPP and ATW) were significantly negatively correlated under low and high $\mathrm{N}$ conditions ( -0.36 and -0.37 respectively), which reflects a trade-off between the two traits both under low and high $\mathrm{N}$ availability. 
Tabel 2. Coefficients of correlations (r) between various physiological and agronomic traits, of the mapping population under low $\mathrm{N}$ and high $\mathrm{N}$ conditions

\begin{tabular}{|c|c|c|c|c|c|c|c|c|c|c|c|c|c|c|c|c|c|}
\hline \multicolumn{2}{|c|}{ Treatments } & \multicolumn{16}{|c|}{ High N } \\
\hline & Traits & $\mathrm{PH}$ & SNPP & LCC & UCC & $\mathrm{tm} 1$ & $\mathrm{t} 1$ & $\mathrm{t} 2$ & te & Vmax & AUC & DTM & TNPP & TYPP & ATW & TDM $\%$ & NUE \\
\hline \multirow{16}{*}{ Low N } & \multirow{2}{*}{$\begin{array}{l}\text { PH } \\
\text { SNPP }\end{array}$} & 0.88 & 0.13 & 0.01 & -0.09 & 0.22 & 0.40 & 0.19 & 0.02 & 0.78 & 0.60 & 0.41 & 0.48 & 0.67 & 0.23 & -0.24 & 0.58 \\
\hline & & 0.08 & 0.51 & -0.26 & -0.22 & 0.42 & 0.41 & 0.24 & -0.02 & 0.41 & 0.29 & 0.23 & 0.46 & 0.36 & -0.22 & -0.20 & 0.33 \\
\hline & LCC & -0.02 & -0.08 & 0.68 & 0.83 & -0.05 & -0.10 & -0.25 & -0.17 & -0.16 & -0.19 & 0.00 & -0.18 & -0.23 & -0.06 & 0.17 & -0.15 \\
\hline & \multirow{2}{*}{$\begin{array}{l}\mathrm{UCC} \\
\mathrm{tm} 1\end{array}$} & -0.16 & -0.19 & 0.82 & 0.75 & -0.07 & -0.15 & -0.20 & -0.14 & -0.21 & -0.24 & -0.05 & -0.17 & -0.29 & -0.18 & 0.20 & -0.19 \\
\hline & & 0.17 & 0.50 & -0.21 & -0.15 & 0.52 & 0.52 & 0.24 & 0.03 & 0.44 & 0.32 & 0.63 & 0.46 & 0.42 & -0.06 & -0.09 & 0.39 \\
\hline & $\mathrm{t} 1$ & 0.21 & 0.51 & -0.09 & -0.16 & 0.65 & 0.56 & 0.57 & 0.00 & 0.62 & 0.47 & 0.57 & 0.53 & 0.52 & -0.01 & -0.15 & 0.52 \\
\hline & $\mathrm{t} 2$ & -0.06 & 0.46 & -0.12 & -0.12 & 0.47 & 0.38 & 0.21 & 0.24 & 0.31 & 0.34 & 0.37 & 0.39 & 0.38 & -0.05 & -0.12 & 0.39 \\
\hline & $\mathrm{Te}$ & 0.06 & 0.23 & -0.03 & -0.08 & 0.11 & -0.06 & 0.32 & 0.69 & 0.07 & 0.14 & 0.12 & 0.04 & 0.04 & -0.02 & -0.07 & 0.06 \\
\hline & Vmax & 0.65 & 0.22 & -0.12 & -0.30 & 0.26 & 0.35 & 0.01 & 0.03 & 0.77 & 0.73 & 0.55 & 0.69 & 0.82 & 0.14 & -0.31 & 0.74 \\
\hline & AUC & 0.60 & 0.27 & -0.15 & -0.30 & 0.32 & 0.31 & 0.11 & 0.25 & 0.94 & 0.75 & 0.43 & 0.57 & 0.70 & 0.05 & -0.24 & 0.62 \\
\hline & DTM & 0.23 & 0.53 & -0.14 & -0.28 & 0.59 & 0.52 & 0.28 & 0.06 & 0.39 & 0.41 & 0.73 & 0.47 & 0.57 & 0.11 & -0.13 & 0.57 \\
\hline & TNPP & 0.23 & 0.54 & -0.13 & -0.26 & 0.40 & 0.39 & 0.38 & 0.16 & 0.52 & 0.53 & 0.53 & 0.85 & 0.74 & -0.37 & -0.27 & 0.65 \\
\hline & TYPP & 0.55 & 0.44 & -0.22 & -0.36 & 0.39 & 0.38 & 0.24 & 0.17 & 0.73 & 0.73 & 0.57 & 0.66 & 0.87 & 0.27 & -0.30 & 0.87 \\
\hline & ATW & 0.46 & -0.11 & -0.11 & -0.15 & 0.05 & 0.10 & -0.18 & 0.01 & 0.27 & 0.25 & 0.10 & -0.36 & 0.38 & 0.73 & -0.04 & 0.23 \\
\hline & TDM\% & -0.06 & -0.13 & 0.19 & 0.18 & 0.03 & 0.12 & -0.06 & -0.03 & -0.21 & -0.18 & 0.11 & -0.20 & -0.21 & 0.01 & 0.34 & 0.07 \\
\hline & NUE & 0.55 & 0.41 & -0.17 & -0.32 & 0.40 & 0.41 & 0.23 & 0.18 & 0.67 & 0.68 & 0.62 & 0.63 & 0.92 & 0.38 & 0.11 & 0.76 \\
\hline
\end{tabular}

Color Key

$-1$

0

PH $=$ Plant Height, SNPP=stem number plant ${ }^{-1}, \mathrm{LCC}=$ Lower leaves chlorophyll content, $\mathrm{UCC}=$ upper leaf chlorophyll content tm1= inflection point in canopy building phase, $\mathrm{t} 1=$ canopy stabilized, $\mathrm{t} 2=$ on set of canopy decline, te= time canopy cover zero, Vmax= maximum canopy cover in percent, AUC= total area under the canopy, DTM= days to maturity, TNPP=Tuber Number Plant ${ }^{-1}$, TYPP $=$ Tuber Yield Plant ${ }^{-1}$, ATW= Average Tuber Weight, TDM\%=Tuber Dry Matter in percent, NUE $=$ Nitrogen use efficiency 


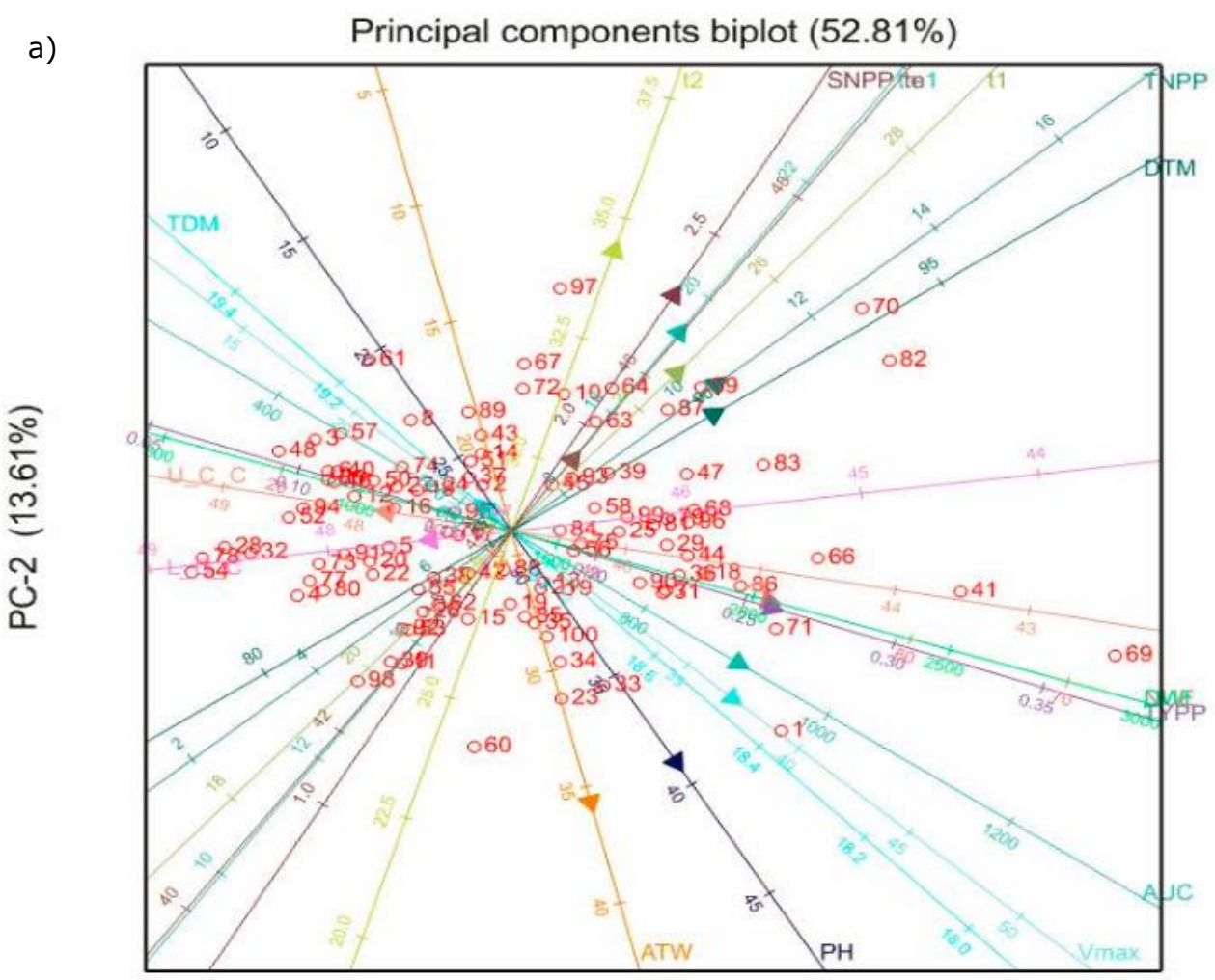

PC-1 (39.2\%)

b Principal components biplot (53.87\%)

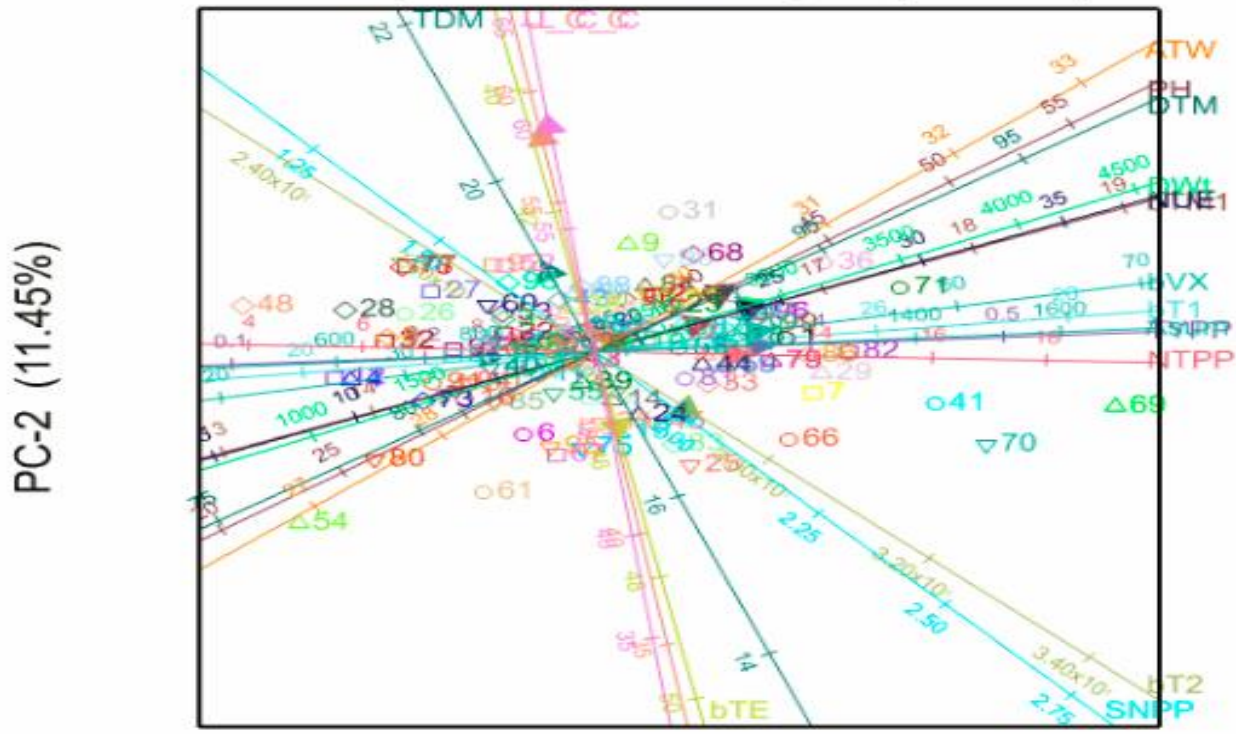

PC-1 (42.43\%)

Figure 3. a) Bi-plot of PC1 viz. PC2 from principal component analysis showing the distribution and similarities among $100 \mathrm{CE}$ potato genotypes under low N, b) Bi-plot of PC1 viz. PC2 from principal component analysis showing distribution and similarities among $100 \mathrm{CE}$ potato genotypes under high $\mathrm{N}$. PH=Plant Height, SNPP=stem number plant ${ }^{-1}, \mathrm{LCC}=$ Lower leaves chlorophyll content, UCC $=$ upper leaf chlorophyll content, $\mathrm{tm} 1=$ inflection point in canopy building phase, $\mathrm{t} 1=$ canopy stabilized, $\mathrm{t} 2=$ on set of canopy decline, te= time canopy cover zero, Vmax = maximum canopy cover in percent, $\mathrm{AUC}=$ total area under the canopy, DTM= days to maturity, TNPP=Tuber Number Plant ${ }^{-1}$, TYPP $=$ Tuber Yield Plant ${ }^{-1}$, ATW= Average Tuber Weight, TDM\%=Tuber Dry Matter in percent, NUE= Nitrogen use efficiency 
Principal component analysis (PCA) is one of the main statistical tools widely used to categorise phenotypic traits into groups based on similarities. The principal component analysis biplots in Figure 3 depict the distribution and similarities of $100 \mathrm{CE}$ potato progeny genotypes including their parents under low (Figure 3a) and high $\mathrm{N}$ conditions (Figure 3b) over all locations combined. In the PCA $52.81 \%$ and $53.87 \%$ of the total variance was explained by PC1 and PC2 together under low and high N conditions, respectively. The angles between vectors in the biplot indicate the level of association between traits. An angle less than $90^{\circ}$ (acute angle) suggests presence of strong positive correlation, an angle greater than $90^{\circ}$ (obtuse angle) suggests a weak correlation. Thus, the biplots point out the genetic relationship between traits. As showed in the biplots a strong correlation was observed between tuber number plant ${ }^{-1}$ (TNPP) and days to maturity (DTM); and NUE and tuber yield plant ${ }^{-1}$ (TYPP) under low N condition. Under high $\mathrm{N}$ there was strong correlation between average tuber weight (ATW), plant height (PH), and days to maturity (DTM); between maximum canopy cover (Vmax), Tuber yield plant ${ }^{-1}$ (TYPP), tuber number plant ${ }^{-1}$ (TNPP) and time at which canopy cover reaches its maximum $(\mathrm{t} 1)$.

\section{QTL detection}

The QTL analysis was done separately for each N level at each location. We have done QTL analysis for all measured traits, and we found QTL for ten traits at three experimental locations (Debre-Tabor, Injibara, and Koga) under low and high $\mathrm{N}$ conditions (summarized in Table 3). A total of 52 QTLs were identified for the ten traits distributed over 13 QTL regions on seven of the 12 linkage groups, of which 28 QTLs were detected under low N while 24 QTLs were detected under high $\mathrm{N}$ conditions. Among the experimental locations, the highest number of QTLs under low and high N conditions together, were detected in Debre-Tabor and Koga (19 QTLs). The identified QTLs accounted for a 11.9 to $37.1 \%$ of the total phenotypic variation for low N, and 15.3 to $38.4 \%$ for high N conditions. Many QTLs were detected repeatedly across locations and $\mathrm{N}$ levels (Table 4). We considered QTLs detected in at least two of the three experimental locations under both low $\mathrm{N}$ and high $\mathrm{N}$ conditions to be constitutive and $\mathrm{N}$-level independent QTLs, and QTLs that were exclusively detected in at least two of the three experimental locations under either high $\mathrm{N}$ or low $\mathrm{N}$ conditions as high $\mathrm{N}$-specific or low-N specific QTLs. Four QTLs were low N specific and 4 QTLs were high N specific, suggesting the presence of QTL $x$ N interaction. The remaining 3 QTLs were detected under both $\mathrm{N}$ conditions. DTM, NUE and TYPP were some of the traits that had low N specific QTLs, while high N specific QTLs were detected for LCC, Vmax and AUC (Table 4). 
The $\mathrm{CxE}$ population is a backcross population with three alleles. Thus, we treated it as a $\mathrm{CP}$ population type (population resulting from a cross between heterogeneous, heterozygous and homozygous diploid parents) in the MapQTL model, because all other models in MapQTL assume a maximum of two alleles.

Table 3. QTLs detected for ten agronomic and physiological traits under low and high $\mathrm{N}$ conditions in the $\mathrm{CxE}$ mapping population.

\begin{tabular}{|c|c|c|c|c|c|c|c|c|}
\hline Traits & Environment & QTL name & $\begin{array}{l}\text { Linkage } \\
\text { Group }\end{array}$ & Peak Marker & LOD & $\begin{array}{l}\text { Peak } \\
\text { position }\end{array}$ & $\begin{array}{l}\text { Interval } \\
(\mathrm{cM})\end{array}$ & $\begin{array}{l}\text { explained. } \\
\text { variation in } \\
(\%)\end{array}$ \\
\hline \multirow{5}{*}{$\begin{array}{l}\text { Area under Canopy } \\
\text { (AUC) }\end{array}$} & IBLN & AUC_I_LN & $\mathrm{V}$ & GP21_2007 & 6.1 & 24.7 & $21-26$ & 26.2 \\
\hline & IBHN & AUC_I_HN & $\mathrm{V}$ & Mando & 6.1 & 26.1 & $24-38$ & 25.9 \\
\hline & KOHN & AUC_K_HN1 & $\mathrm{V}$ & Mando & 5.0 & 26.1 & $24-38$ & 17.5 \\
\hline & DTHN & AUC_D_HN & $\mathrm{V}$ & PotSNP1146 & 5.3 & 43.5 & $38-47$ & 23 \\
\hline & KOHN & AUC_K_HN2 & $\mathrm{V}$ & PotSNP1143 & 4.5 & 47 & $43-50$ & 20.1 \\
\hline \multirow{7}{*}{$\begin{array}{l}\text { Days to maturity } \\
\text { (DTM) }\end{array}$} & DTLN & DTM_D_LN1 & IV & PotSNP51 & 4.9 & 65.7 & $60-72$ & 21.7 \\
\hline & IBLN & DTM_I_LN1 & $\mathrm{V}$ & Mando & 4.6 & 26.1 & $24-38$ & 14.3 \\
\hline & IBHN & DTM_I_HN & $\mathrm{V}$ & SPUD237 & 5.6 & 31.1 & $26-38$ & 24.1 \\
\hline & IBLN & DTM_I_LN2 & V & PotSNP1143 & 7.0 & 47 & $43-50$ & 29.2 \\
\hline & DTHN & DTM_D_HN1 & $\mathrm{V}$ & Myb_t10 & 4.7 & 49.6 & $46-54$ & 16.7 \\
\hline & DTLN & DTM_D_LN2 & V & PotSNP43 & 4.8 & 51.6 & $46-56$ & 16.6 \\
\hline & DTHN & DTM_D_HN2 & IX & E32M51-1c9 & 4.5 & 54.6 & $51-57$ & 15.2 \\
\hline \multirow{6}{*}{$\begin{array}{l}\text { Lower leaf } \\
\text { chlorophyll (LCC) }\end{array}$} & KOLN & LCC_K_LN & I & PotSNP1037 & 4.9 & 44.5 & $42-47$ & 21.4 \\
\hline & DTLN & LCC_D_LN1 & I & E32M61-18e13 & 5.8 & 45.3 & $42-48$ & 22 \\
\hline & DTLN & LCC_D_LN2 & II & PotSNP1 & 4.0 & 51.1 & $46-57$ & 15.8 \\
\hline & DTHN & LCC_D_HN & II & PotSNP807 & 5.1 & 51.6 & $46-57$ & 22.3 \\
\hline & KOHN & LCC_K_HN & II & PotSNP807 & 9.4 & 51.6 & $49-57$ & 37.1 \\
\hline & DTLN & LCC_D_LN3 & VIII & E32M51-15h8 & 5.2 & 81.3 & $77-84$ & 20.1 \\
\hline \multirow{4}{*}{$\begin{array}{l}\text { Nitrogen use } \\
\text { efficiency (NUE) }\end{array}$} & DTLN & NUE_D_LN1 & IV & PotSNP51 & 5.3 & 65.7 & $60-72$ & 23.2 \\
\hline & KOHN & NUE_K_HN & $\mathrm{V}$ & PotSNP573 & 5.5 & 15.5 & $0-21$ & 23.7 \\
\hline & DTLN & NUE_D_LN2 & $\mathrm{V}$ & Mando & 4.9 & 26.1 & $24-38$ & 16.4 \\
\hline & KOLN & NUE_K_LN & $\mathrm{V}$ & SPUD237 & 8.11 & 31.1 & $26-38$ & 33.1 \\
\hline \multirow[t]{8}{*}{ Plant height $(\mathrm{PH})$} & IBLN & PH_I_LN1 & V & PBSQ & 5.4 & 24.4 & $21-26$ & 18.7 \\
\hline & IBHN & PH_I_HN1 & V & GP21_2007 & 6.24 & 24.7 & $21-26$ & 20.5 \\
\hline & KOLN & PH_K_LN1 & $\mathrm{V}$ & GP21_2007 & 7.14 & 24.7 & $21-27$ & 22.5 \\
\hline & KOHN & PH_K_HN1 & V & GP21_2007 & 5.7 & 24.7 & $24-38$ & 16.6 \\
\hline & IBLN & PH_I_LN2 & $\mathrm{V}$ & Myb_t10 & 4.6 & 49.6 & $46-54$ & 20.4 \\
\hline & IBHN & PH_I_HN2 & $\mathrm{V}$ & Myb_t10 & 5.2 & 49.6 & $46-54$ & 22.8 \\
\hline & $\mathrm{KOHN}$ & PH_K_HN2 & $\mathrm{V}$ & E32M61-9h5 & 5.3 & 61.3 & $58-70$ & 15.3 \\
\hline & KOLN & PH_K_LN2 & V & E32M61-9h5 & 6.2 & 61.3 & $58-70$ & 26.5 \\
\hline $\begin{array}{l}\text { Stem number/plant } \\
\text { (SNPP) }\end{array}$ & KOLN & SNPP_K_LN & $\mathrm{V}$ & E32M61-9h5 & 4.6 & 61.3 & $58-70$ & 20.5 \\
\hline \multirow{4}{*}{$\begin{array}{l}\text { Tuber number/plant } \\
\text { (TNPP) }\end{array}$} & DTHN & TNPP_D_HN & V & SPUD237 & 5.4 & 31.1 & $26-38$ & 23.3 \\
\hline & DTLN & TNPP_D_LN & V & SPUD237 & 6.9 & 31.1 & $26-38$ & 28.9 \\
\hline & IBHN & TNPP_I_HN & $\mathrm{V}$ & SPUD237 & 5.8 & 31.1 & $26-38$ & 24.9 \\
\hline & KOHN & TNPP_K_HN & $\mathrm{V}$ & SPUD237 & 5.3 & 31.1 & $26-38$ & 23.1 \\
\hline
\end{tabular}




\begin{tabular}{|c|c|c|c|c|c|c|c|c|}
\hline Traits & Environment & QTL name & $\begin{array}{l}\text { Linkage } \\
\text { Group }\end{array}$ & Peak Marker & LOD & $\begin{array}{l}\text { Peak } \\
\text { position }\end{array}$ & $\begin{array}{l}\text { Interval } \\
(\mathrm{cM})\end{array}$ & $\begin{array}{l}\text { explained. } \\
\text { variation in } \\
(\%)\end{array}$ \\
\hline & KOLN & TNPP_K_LN & $\mathrm{V}$ & SPUD237 & 6 & 31.1 & $26-38$ & 25.7 \\
\hline \multirow{7}{*}{$\begin{array}{l}\text { Tuber yield/plant } \\
\text { (TYPP) }\end{array}$} & KOHN & TYPP_K_HN & V & PBSQ & 7.4 & 24.4 & $21-26$ & 30.5 \\
\hline & KOLN & TYPP_K_LN1 & $\mathrm{V}$ & Mando & 6.3 & 26.13 & $24-38$ & 21.5 \\
\hline & DTLN & TYPP_D_LN & V & SPUD237 & 8.2 & 31.1 & $26-38$ & 33.2 \\
\hline & IBLN & TYPP_I_LN & $\mathrm{V}$ & SPUD237 & 4.7 & 31.1 & $26-38$ & 20.7 \\
\hline & IBHN & TYPP_I_HN & V & PotSNP1143 & 5.3 & 47 & $43-50$ & 22.9 \\
\hline & KOLN & TYPP_K_LN2 & $\mathrm{V}$ & E32M61-9h5 & 4.6 & 61.3 & $58-70$ & 20.2 \\
\hline & DTHN & TYPP_D_HN & VII & PotSNP788 & 7.4 & 42.1 & $39-49$ & 26.6 \\
\hline \multirow{4}{*}{$\begin{array}{l}\text { Upper leaf } \\
\text { chlorophyll (UCC) }\end{array}$} & DTLN & UCC_D_LN1 & I & STM5136 & 4.5 & 23 & $18-27$ & 16.5 \\
\hline & DTLN & UCC_D_LN2 & II & PotSNP1111 & 4.6 & 112.5 & $106-115$ & 16.7 \\
\hline & DTLN & UCC_D_LN3 & $\mathrm{V}$ & potSNP90 & 6.0 & 51.6 & $46-56$ & 13.2 \\
\hline & DTLN & UCC_D_LN4 & VII & potSNP542 & 5.5 & 89.2 & $86-91$ & 11.9 \\
\hline \multirow{6}{*}{$\begin{array}{l}\text { Maximum canopy } \\
\text { cover (Vmax) }\end{array}$} & IBLN & Vmax_I_LN & V & Mando & 5.9 & 26.13 & $24-28$ & 25.2 \\
\hline & IBHN & Vmax_I_HN & V & Mando & 5.4 & 26.13 & $24-38$ & 23.4 \\
\hline & KOHN & Vmax_K_HN1 & V & Mando & 4.5 & 26.13 & $24-38$ & 15.8 \\
\hline & KOLN & Vmax_K_LN & V & GP21_2007 & 9.8 & 24.7 & $21-27$ & 38.4 \\
\hline & DTHN & Vmax_D_HN & V & PotSNP1146 & 5.3 & 43.5 & $38-47$ & 23.2 \\
\hline & KOHN & Vmax_K_HN2 & V & PotSNP1143 & 4.7 & 47 & $43-49$ & 20.9 \\
\hline
\end{tabular}

DTLN = Debre-Tabor low N, DTHN = Debre-Tabor high N, IBLN = Injibara low N, IBHN = Injibara high N, KOLN = Koga low N, KOHN = Koga high N, QTL names are given as trait name followed by location and the N-levels e.g AUC_I_LN: Area under canopy (AUC); Location, Injibara (I); N-Level (LN), low N

Table 4. List of agronomic and physiological traits for which QTLs were found in more than one location under low or high $\mathrm{N}$ specific conditions and under both $\mathrm{N}$ level conditions

\begin{tabular}{lllllll}
\hline Traits & $\begin{array}{c}\text { High N } \\
\text { specific QTLs }\end{array}$ & $\begin{array}{c}\text { Low N } \\
\text { specific QTLs }\end{array}$ & $\begin{array}{c}\text { Low and } \\
\text { high N } \\
\text { conditions }\end{array}$ & $\begin{array}{c}\text { Linkage } \\
\text { group }\end{array}$ & Interval(cM) & Locations \\
\hline PH & $\checkmark$ & $\checkmark$ & V & $21-27$ & DT and Injibara \\
LCC & $\checkmark$ & II & $46-57$ & DT and Koga \\
LCC & $\checkmark$ & & I & $42-48$ & Koga and Injibara \\
AUC & $\checkmark$ & & V & $24-38$ & DT and Injibara \\
AUC & $\checkmark$ & & V & $38-50$ & DT and Koga \\
Vmax & & & V & $38-49$ & DT and Koga \\
Vmax & & & V & $24-38$ & Koga and Injibara \\
DTM & & $\checkmark$ & V & $43-56$ & DT and Injibara \\
TNPP & & & V & $26-38$ & DT and Koga \\
TYPP & & $\checkmark$ & V & $24-38$ & DT, IB and Koga \\
NUE & & $\checkmark$ & V & $24-38$ & DT and Koga \\
\hline
\end{tabular}

$\mathrm{PH}=$ Plant height, $\mathrm{LCC}=$ lower leaf chlorophyll content, AUC $=$ Area under the canopy curve, Vmax = Maximum canopy cover, $\mathrm{DTM}=$ Days to maturity, TNPP $=$ Tuber number plant ${ }^{-1}$, TYPP $=$ Tuber yield plant ${ }^{-1}$, NUE $=$ Nitrogen use efficiency. DT $=$ DebreTabor, IB= Injibara

Consequently, the genotypes were coded with "ab x cd", where a and b represent the alleles of parent $\mathrm{C}$ and $\mathrm{c}$ and $\mathrm{d}$ represent the alleles of parent $\mathrm{E}$ with possible genotypes ac, ad, bc, and 
bd. In our backcross population, one of the alleles derived from the $\mathrm{C}$ parent is in fact identical to one of the E-derived alleles, but haplotype information is not available, so it was not possible to distinguish which allele is which. For the QTLs identified for days to maturity (DTM) on linkage group IX under low and on linkage group V under high $\mathrm{N}$ conditions at Debre-Tabor the ' $c$ ' allele from the E-parent most likely contributes to late maturity type in this population (Figure 4a and b). The QTL identified on chromosome V with peak marker SPAD237 for tuber number plant ${ }^{-1}$ detected under both $\mathrm{N}$ conditions showed a similar positive contribution of the $\mathrm{E}$ parent-derived allele (Figure $4 \mathrm{c}$ and d). For tuber yield plant ${ }^{-1}$, however, a specific combination of C- and E-derived alleles was linked to high tuber yields (Figure 4e and f) for each of the QTLs.
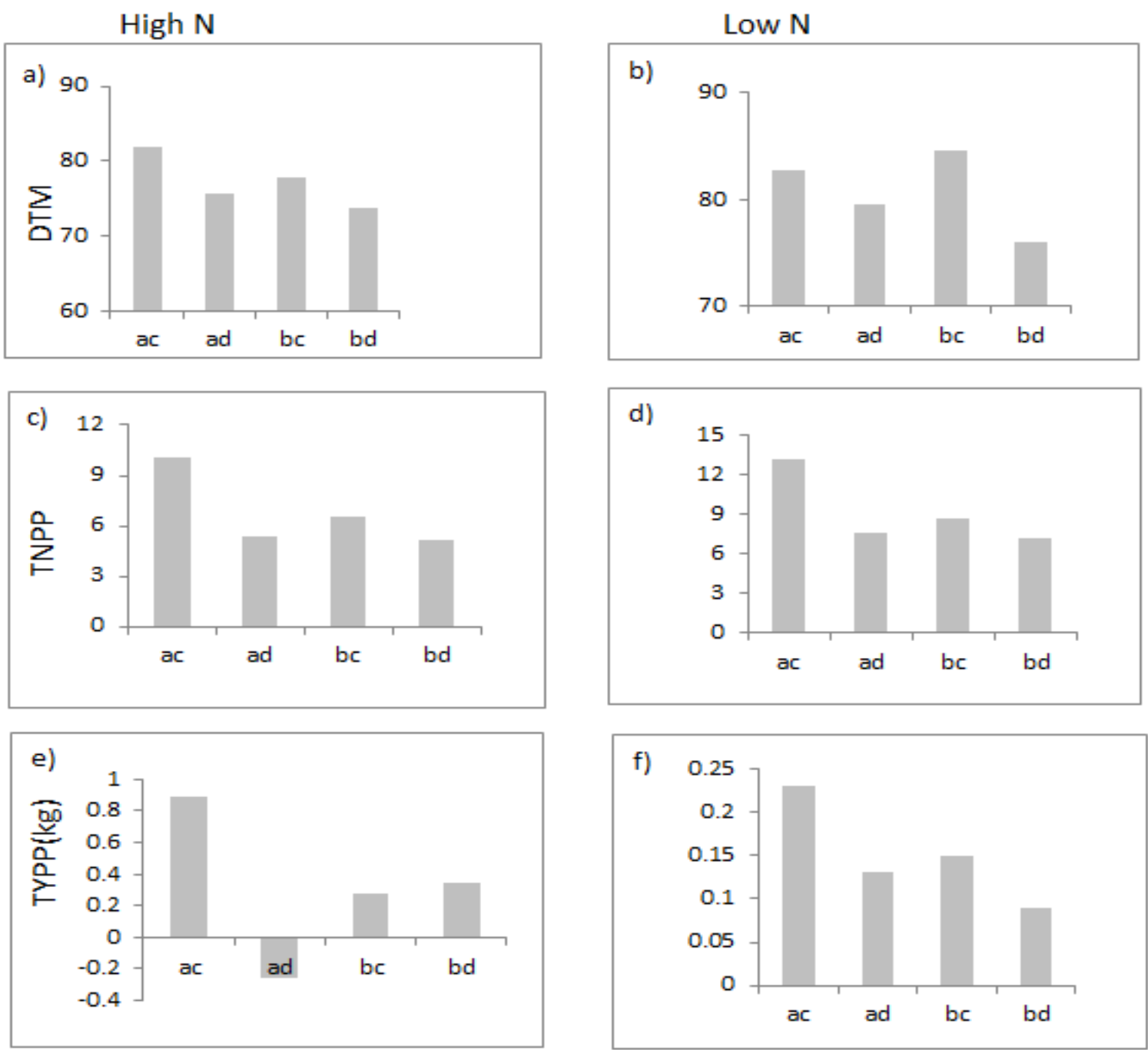

Figure 4. Expected mean of CE offspring for some selected trait QTLs in different genomic locations under high and low $\mathrm{N}$ condition in Debre-Tabor.a) DTM_D_HN2 for marker E32M51-1c9 on linkage group IX, b) DTM_D_LN2 for marker PotSNP43 on linkage group V, c) TNPP_D_HN for marker SPUD237 on linkage group V, d) TNPP_D_LN for marker SPUD237 on linkage group V, e) TYPP_D_HN for marker PotSNP788 on linkage group VII, f) TYPP_D_LN for marker SPUD237on linkage group $\mathrm{V}$ 
The identification of similar QTLs for tuber number plant $^{-1}$ with similar allele contributor under both $\mathrm{N}$ conditions suggests that the same gene effect is responsible for these QTLs. However, the QTLs identified for tuber yield plant ${ }^{-1}$ had different allele contributors for low and high $\mathrm{N}$ levels. Overall, as shown above, the CE progeny that had the alleles corresponding to ' $a$ ' from the female parent $\mathrm{C}$ and allele ' $\mathrm{c}$ ' from the male parent $\mathrm{E}$ showed a high score, and allele 'c' was responsible for the high value in most listed traits under low and high $\mathrm{N}$ conditions.

Of the 13 QTL regions, four genomic regions, i.e. on linkage group V between 21-38cM, 38$56 \mathrm{cM}$, and $58-70 \mathrm{cM}$, and on linkage group IV between $60-72 \mathrm{cM}$ contained QTL regions accumulating QTLs for more than one trait under different $\mathrm{N}$ conditions and locations (Figure 5). The peak markers for the QTL regions on chromosome V were more than 20cM apart, which might indicate these are indeed independent QTL regions. However, we do not have sufficient marker information and recombinants to confirm this. The QTLs for AUC, PH, Vmax, DTM, TNPP, TYPP and NUE co-localized between 21 and 38cM on linkage group V. QTLs for DTM and PH under both N conditions, and Vmax, AUC and TYPP under high N conditions co-located between 38 and 56cM on linkage group V. This co-localization of QTLs of different traits in the same chromosomal regions suggests the existence of physiological and/or genetic relationships between these traits. NUE and DTM under low N conditions shared the same QTL region $(60-72 \mathrm{cM})$ on linkage group IV, explaining $23.2 \%$ and $21.7 \%$ of the total phenotypic variation of NUE and DTM respectively. In total, $77 \%$ of the detected QTLs were located on linkage group V, grouped into 4 cluster regions. From these 4 QTL cluster regions, the region between 21 and $38 \mathrm{cM}$ accumulated most QTLs for NUE and related traits. 
CE1

CE2

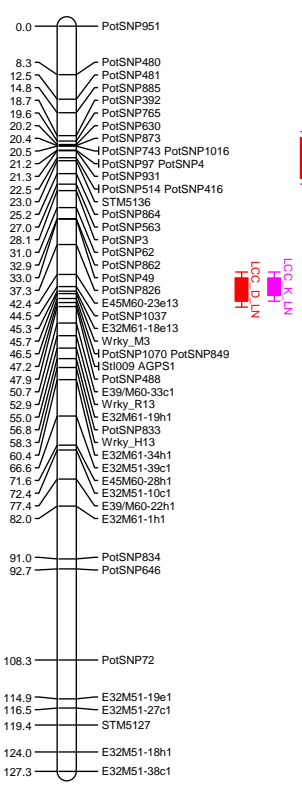

CE5
CE4

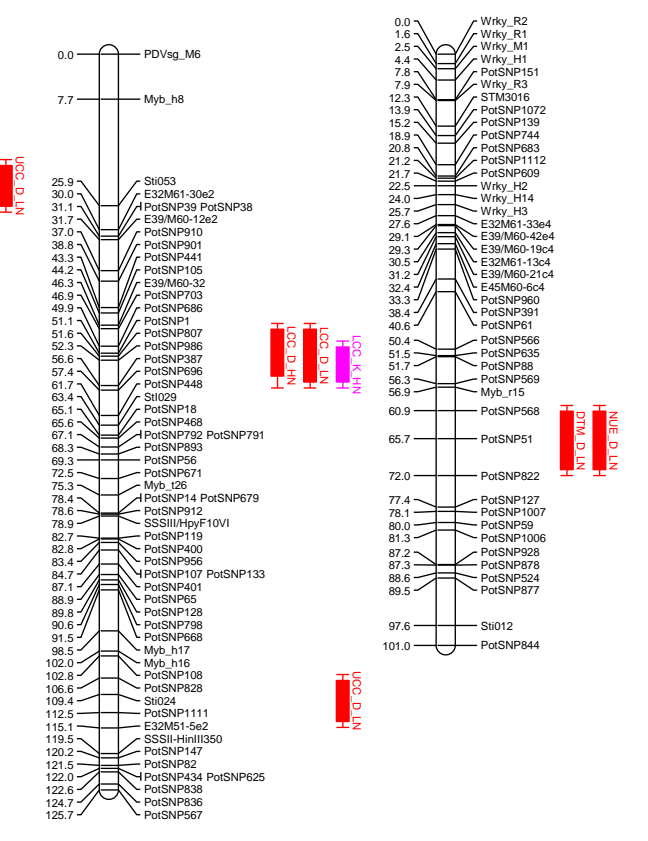

CE7

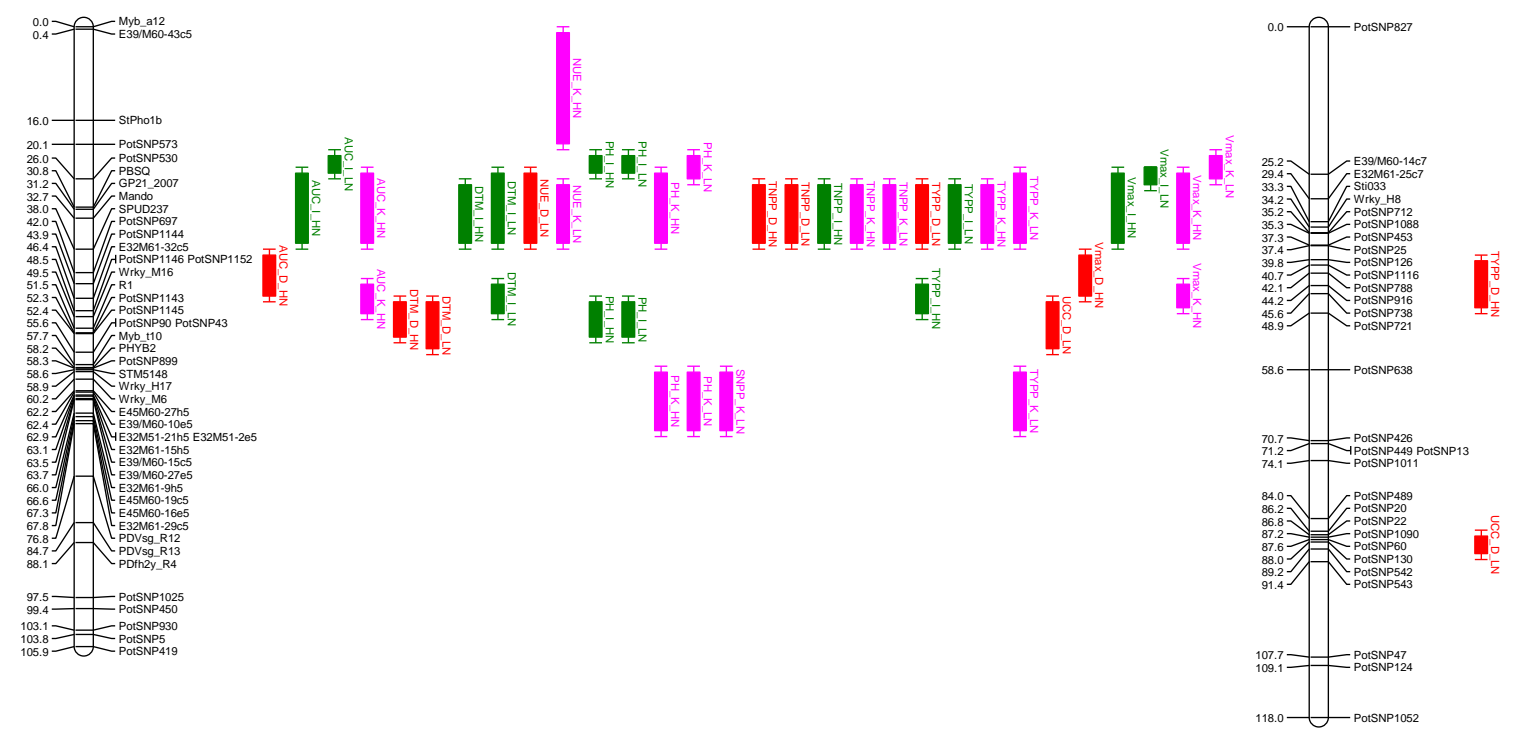



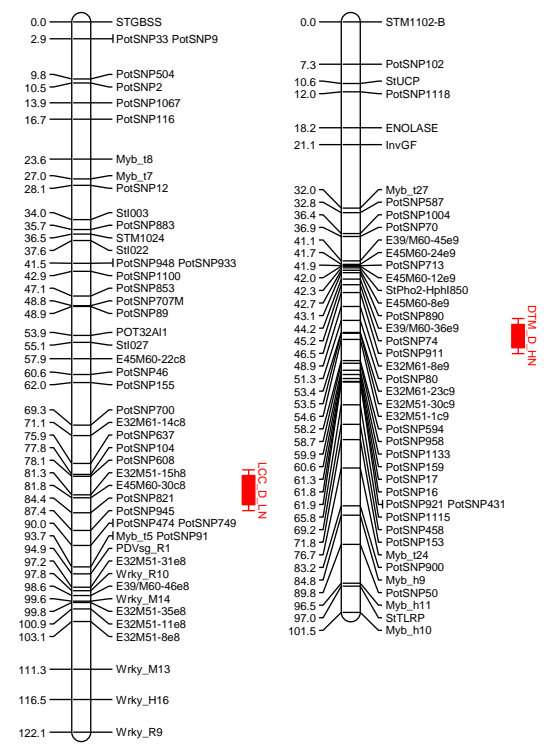

Figure. 5 Locations of the QTLs on the CE integerated map. Only chromosomes (linkage groups) with QTL are presented. The numbers on the left side are genetic distances in centimorgans (cM); marker names and locations are given on the right side. QTLs are presented at the right side as vertical bars with trait names in different colors for different locations (red: Debre-Tabor; green:Injibara; Purple: Koga). QTL names are given as trait name followed by locations and N-levels. e.g AUC_I_LN (Area under canopy curve (AUC); Location, Injibara (I); N-Level(LN) or low N .Abbreviations for trait names: Area under canopy(AUC), Days to maturity (DTM), Lower leaf chlorophyll (LCC), Nitrogen use efficiency (NUE), Plant Height (PH), stem number(SNPP), Tuber number (TNPP), Tuber yield (TYPP), Upper leaf chlorophyll (UCC), Maximum canopy cover (Vmax).

\section{Discussion}

Breeding for higher yields in crops can be successful via the monitoring and selection for the component physiological traits that determine biomass partitioning and production, and the identification of QTLs that control the heritable variation of these traits (Tuberosa et al., 2008). This is especially true for improving yields under stressful conditions, like low nutrient availability. In the present study, the CxE backcross diploid potato population was evaluated under field conditions to identify QTLs that contribute to NUE and related traits under low and high $\mathrm{N}$ availability in potato.

The pooled analysis of variance showed significant differences between genotypes, locations, $\mathrm{N}$ levels, and their interaction for most measured traits. Nitrogen availability affects various physiological processes and morphological traits of the potato crop. TYPP, TNPP, and Vmax were among the traits that were strongly affected by $\mathrm{N}$ level in our study. Vos and Biemond (1992) reported that $\mathrm{N}$ availability affects the rate of canopy development, leaf appearance, final leaf size and rate of photosynthesis. $\mathrm{N}$ supply was also suggested to affect onset of 
tuberization, final tuber yield and harvest index (Ewing \& Struik, 1992; Vos, 1995; Vos \& MacKerron, 2000; Ospina et al, 2014). In our study, area under the canopy curve (AUC) was significantly affected by the level of applied N. Similarly, Grindlay (1997) and Ospina et al. (2014) reported that limitation in $\mathrm{N}$ supply affects canopy cover negatively, resulting in reduction in the amount of solar radiation intercepted and the overall photosynthetic capacity.

\section{QTL identification}

To date, only a few studies report QTLs regulating potato responses to abiotic stress (Anithakumari et al., 2011 2012; Khan et al., 2014; Ospina, 2016). Our study detected multilocation as well as multi-treatment QTLs for NUE and NUE-related traits. Most of the 52 identified QTLs explained more than $15 \%$ of the total phenotypic variation of the trait.

Four genomic regions which harbor QTLs affecting more than one trait were identified on linkage group $\mathrm{V}$ and on linkage group IV under different $\mathrm{N}$ levels and locations. AUC, PH, Vmax, DTM, TNPP, TYPP and NUE QTLs co-localized on linkage group V between 21-38cM and most of the QTLs had the same peak markers, indicating that a single gene with pleiotropic effects may contribute to this cluster of traits or that the measured traits are physiologically and/or morphologically linked (El-Soda, Martin, and Boer et al., 2014). These traits had a strong positive correlation with NUE and with each other under both $\mathrm{N}$ conditions. The colocalization of yield and canopy traits is in line with Haverkort et al. (1991) and Vos (2009) who reported a strong correlation of canopy cover with intercepted photosynthetically active radiation and tuber yield. The strong positive association of traits with NUE and co-localization in the same QTL region make the traits interesting for breeders to consider them as a selection criterion to improve NUE in the potato breeding programs. This region was found previously to harbour QTLs for multiple traits under different abiotic stresses and normal growing conditions. Anithakumari et al. (2012) in their drought tolerance study found that the same region was associated with shoot fresh weight, tuber number, tuber weight and root length under drought stress and recovery conditions. QTLs associated with foliage maturity and late blight resistance were also identified in this region under normal potato growing conditions (Visker et al., 2005; McCord et al., 2011). Khan et al. (2014) in their drought tolerance study also reported that this QTL region harboured QTLs for plant height, chlorophyll content, tuber number and tuber weight under drought and well-watered conditions, indicating that the region is a potential QTL region for most important agronomic and physiological traits of potato. This region of linkage group $\mathrm{V}$ in the potato genome is strongly linked to early maturity and initiation of tuberization, for which the CDF1 gene was shown to be responsible (Kloosterman 
et al., 2013). In our study, earliness has a profound influence on NUE regardless of N level, and our results are in line with the findings of Zebarth et al. (2004) and Ospina et al. (2014). For the most effective use of the QTLs in this region for NUE improvement programs in potato, it may be necessary to see whether the NUE QTL effects are not caused by variation in the CDF1 gene, and to identify the genes that regulate the NUE related traits. If the genes are different from CDF1 gene but linked with it, disentangling the earliness gene from the genes that regulate NUE and other NUE related traits may be helpful or even required to improve these traits.

Additional QTLs for DTM and PH under both N conditions, Vmax, AUC and TYPP under high $\mathrm{N}$ conditions, and UCC under low $\mathrm{N}$ condition (in total about 11 QTLs) were co-located between 38-56cM on linkage group V. Previously QTLs associated with foliage traits were also identified in this region: QTLs for fresh biomass (Anithakumari et al., 2011), plant height, shoot fresh weight and shoot dry weight under drought stress and recovery condition (Anithakumari et al., 2012), for fresh and dry harvest index and stem diameter under drought and well-watered conditions (Khan et al., 2014) were identified in this region. Most of the QTLs detected in this region are more than 20cM downstream of the CDF1 gene, and may constitute different loci, independent of the earliness locus. This region may be used as a potential source of genes for NUE improvement. The QTL region at 58-70cM on linkage group V harbored 4 QTLs for PH, SNPP and TYPP particularly under low N conditions in Koga. Similarly, Anithakumari et al. (2012) detected QTLs for plant height, stem number, shoot fresh weight and shoot dry weight in this region under drought stress conditions. These QTLs related to growth and yield under both drought stress and $\mathrm{N}$ deficiency conditions are more likely to be independent of maturity, and are potential targets for improving growth under marginal conditions like the test-sites of our trials in Ethiopia. In addition to the multi-QTL locus on chromosome V, NUE and DTM also shared the same QTL region on linkage group IV under low $\mathrm{N}$ conditions. The strong phenotypic correlation between NUE and DTM and the colocalization of their QTLs at several regions indicates that NUE and DTM are genetically strongly related.

In general, the strong positive correlation of TYPP and NUE with DTM, Vmax, AUC, PH and their coinciding QTLs as reported in this study highlight the genetic and physiological relationship between these traits. Notably, the clustered QTLs had a similar additive effect: Parent E contributed the responsible allele for high performance values for the above mentioned traits under low and high $\mathrm{N}$ conditions. The traits may be causally related and thus could be 
simultaneously improved in potato breeding. Especially linkage group V may be enriched with the N metabolism genes. Coincidence of QTL for traits with QTL effects in the same direction may not provide conclusive evidence, but it offers additional evidence that the two traits are functionally associated (Thumma et.al., 2001). The ultimate evidence that two correlated traits are causally correlated may require identification of the putative candidate genes underlying the traits.

\section{QTL x Environment interaction}

Quantitative traits are influenced by the environment and have a tendency to express variable degrees of Genotype $\times$ Environment Interaction (GEI). The analysis of variance in this study indicated presence of GEI. However, the $\mathrm{G} \times \mathrm{N}$ level interaction was low as compared $\mathrm{G} \times$ location interaction. Gallais and Coque (2005) in their maize NUE genetic variation study reported that although the genotype by $\mathrm{N}$ level interaction was low, different traits and genes may underlie the genetic variation in NUE at high $\mathrm{N}$ and low $\mathrm{N}$ level; the variation at high $\mathrm{N}$ was mainly due to variation in $\mathrm{N}$ uptake while at low $\mathrm{N}$ level both components of NUE had a significant contribution to the total NUE variation. This implies that the genes that control NUE at low $\mathrm{N}$ may be different from those at high $\mathrm{N}$ conditions. This may be reflected in QTL $\mathrm{x}$ Environment interaction (QEI). GEI is determined by all the trait-underlying genes of all QTLs combined, while QEI indicates the interaction of a single QTL with the environment. The presence of highly significant GEI typically may or may not indicate the presence of QEI (Wei et al., 2012). Most of the identified QTLs were present only under low $\mathrm{N}$ or high $\mathrm{N}$ conditions, and only some of them under both $\mathrm{N}$ conditions, at least over two experimental locations. QTLs identified at either low $\mathrm{N}$ or high $\mathrm{N}$ condition are $\mathrm{N}$ level dependent, adaptive QTLs, while QTLs identified under both $\mathrm{N}$ condition are $\mathrm{N}$ level independent, constitutive QTLs. The occurrence of adaptive QTLs specific for $\mathrm{N}$ level suggests the presence of QTL $\mathrm{x} N$ interaction. Our study was conducted in three different locations, and two production seasons (rainfed and irrigation production) which are different in several environmental factors (altitude, temperature, soil type and water availability) under low and high $\mathrm{N}$ conditions. This difference in environmental factors will have contributed to QEI. However, the QTLs identified for TNPP, TYPP, NUE, Vmax, and AUC were shared in both rainfed and irrigation production seasons, suggesting that these QTLs are not production season specific. The difference in number of QTLs between location was almost similar to the difference in number of QTL between $\mathrm{N}$ levels, indicating QTL $\mathrm{x}$ location and QTL $\mathrm{x} \mathrm{N}$ level interaction had similar contribution to the total QEI. 


\section{Implications for breeding}

In our study, most measured physiological and agronomic traits had a strong correlation with NUE and co-localized in the same QTL regions. This coincidence of QTLs for NUE with other NUE related traits would suggest the NUE related traits played a role in the NUE performance of potato genotypes (have a causal relationship with NUE). However, to have evidence for causal relationship, identification of the genes that regulate the expression of these correlated traits should be considered in the future study. Moreover, the result suggested that when we simultaneously improve NUE and NUE related traits undesirable genetic linkage and pleiotropy should be considered in the future breeding. Fine mapping and identification of candidate genes is also required to obtain more information about the above mentioned QTL regions simultaneously controlling NUE and related traits. This study can be considered as a first exploratory work on the genetic relation of NUE and related traits under low and high N condition in potato. Most of the QTLs identified in this study were different across environments, suggesting the use of these QTLs would be difficult in breeding or general stability. To verify the identified QTLs in this study are consistently expressed in different environments and to use in breeding for general stability, multiple field trials will be required in different environments. 
Chapter 4

\section{Genome-wide association mapping of nitrogen use efficiency and related traits in potato under contrasting nitrogen regimes}

Baye Berihun Getahun ${ }^{1,2}$, Ernest Aliche ${ }^{1,2}$, Richard RGF Visser ${ }^{1,2}$, C. Gerard van der Linden ${ }^{1,2}$

1Graduate School of Experimental Plant Sciences, Wageningen University \& Research PO Box 386, 6700 AJ Wageningen, The Netherlands

2 Plant Breeding, Wageningen University \& Research, PO Box 386, 6700 AJ Wageningen, The Netherlands 


\begin{abstract}
Nitrogen use efficiency (NUE) is a complex agronomic trait controlled by multiple genes. Deciphering the genetic basis of complex traits like NUE requires the linking of physiological functions and agronomic traits to DNA markers. A genome-wide association mapping study (GWAS) was conducted in potato to identify markers associated with NUE and NUE related agronomic and physiological traits under low $\mathrm{N}$ and high $\mathrm{N}$ conditions. The study was conducted in two production systems (irrigation and rainfed) at Debre-Tabor, Injibara and Koga, Ethiopia in 2013 and 2015. The association panel comprised of 70 tetraploid European potato cultivars and progenitor lines genotyped using markers from a 20k Infinium SNP array. The cultivar panel showed large variation under both $\mathrm{N}$ conditions for most traits, including maximum canopy cover, total area under the canopy curve, days to maturity, tuber number plant $^{-1}$, tuber yield plant ${ }^{-1}$, and tuber dry matter. Marker-trait associations were discovered using the R-software package GWASpoly and for each trait four GWAS were conducted based on the additive, simplex-dominant, duplex-dominant and general genetic models. Most NUE and NUE related trait QTLs were identified using dominant genetic models. A total of 77 marker trait associations were identified for NUE and NUE related agronomic and physiological traits. The effect of production season on QTL $x$ Environment interaction was greater than the effect of $\mathrm{N}$ levels for most NUE related traits. Multi-trait genomic regions that harboured significant marker-trait associations for NUE and NUE related traits were found on chromosome III, V and VI.
\end{abstract}




\section{Introduction}

Nitrogen is one of the most essential agricultural inputs for high crop production and productivity in the world. Application of mineral $\mathrm{N}$ fertilizer is the main driver for restoring soil $\mathrm{N}$ and high crop yields (Hirel et al., 2011). A dramatic increase has occurred in crop yield through global utilization of synthetic $\mathrm{N}$, however in the last decades the consumption rate of $\mathrm{N}$ fertilizer and the crop yield increase were not proportional. In the last 40 years, the amount of mineral $\mathrm{N}$ application to cultivated crops increased 7.4 -fold, while the overall crop yield increase was only 2.4-fold (Tilman et al., 2002). According to the Food and Agriculture Organization (FAO), the growth rate of cereal crop yield has been relatively slow (1\% annually since the mid-1980s); especially in developed countries the increase of crop yield is close to non-existent (Fischer et al., 2009). The recent increase of $\mathrm{N}$ fertilizer use has not only lead to only minor yield gains and reduced nitrogen use efficiency (NUE) of the crops, but also to serious environmental problems (Cassman et al.,2003; David et al., 2010).

Considering these recent crop yield trends together with the forecasted population growth and environmental strain caused by synthetic $\mathrm{N}$ fertilizer use, breeding efforts need to focus on traits related to yield stability and maintenance with suboptimal $\mathrm{N}$ availability. Breeding for $\mathrm{N}$ efficient crop cultivars is a sustainable strategy towards this goal. However, NUE is a complex trait controlled by a large number of genes. Deciphering the genetic basis of complex traits like NUE requires the linking of physiological functions and agronomic traits to DNA markers (Prioul et al., 1997; Hirel et al., 2007b). Knowledge about the genes and molecular mechanisms involved in $\mathrm{N}$ metabolism and the traits that are affected by low $\mathrm{N}$ input is important to elucidate the genetic basis of potato NUE and discover the genes underlying the traits.

Improvement and identification of genes responsible for the genetic variation of agronomically important traits like NUE is not an easy task, as these traits are quantitatively inherited (Neumann et al., 2011). The indirect selection of genetic determinants that are identified by estimating the genomic positions and effects of quantitative trait loci (QTL) using marker assisted selection (MAS) facilitates the development of new crop varieties with desirable component traits (Stich et al., 2008).

The most common genetic mapping approach in cultivated plant species involves bi-parental segregating populations derived from parents with contrasting phenotypes and genotypes. However, such mapping populations represent only a small proportion of all possible allele combinations (Simko and $\mathrm{Hu}, 2008$ ). These also have relatively low resolution: Bi-parental 
QTL mapping detects genomic regions with QTL for a trait with a precision ranging from a few to several tens of centiMorgans (cM), and this chromosomal region may harbour several hundreds to thousands of genes (Ingvarsson et al., 2010). Large size mapping populations improve mapping resolution but require time to evaluate in multiple environments in order to obtain reliable phenotypic information (Mather et al., 2004; Jun et al., 2008). In contrast to linkage mapping, association mapping (AM) is an approach that identifies the relationship between phenotypic variation and genetic polymorphisms in collections of genotypes, without the need for developing bi-parental populations (Simko et al., 2008). According to Zhu et al. (2008), the existing genetic variation of complex traits in for instance natural populations can be investigated using association mapping approaches with high mapping resolution. Association mapping detects QTLs by exploring marker-trait associations resulting from linkage disequilibrium (LD) between markers and trait-functional polymorphisms across a panel of diverse individuals (Zhu et al. 2008). It deals better than linkage analysis using segregating bi-parental populations with tetraploid, non-inbred crops (Li et al., 2010) like potato, for which tetrasomic inheritance is complex (Malosetti et al., 2007).

Cultivated potato is a vegetatively propagated auto-tetraploid $(2 n=4 x=48)$ crop. Association mapping studies in potato have been performed for disease resistance and quality trait mapping. Gebhardt et al. (2004) mapped late blight resistance and plant maturity, Simko et al. (2004 a) Verticillium resistance, Malosetti et al. (2007) late blight resistance, and D'hoop et al. (2008) used association mapping for quality traits. In this study, a genome wide association mapping approach was used to identify markers associated with NUE and NUE-related agronomic and physiological traits under low $\mathrm{N}$ and high $\mathrm{N}$ environment, which may help in further identification of candidate genes that may be useful for allele mining in potato germplasm, and marker assisted selection in the NUE improvement of potato.

\section{Materials and Methods}

Population description. A set of 70 tetraploid potato cultivars and progenitor lines with release dates ranging from 1908 to 2011 (Berloo et al., 2007) for commercial production in Europe were used in this study, referred to here as the association panel (Supplementary Table 1). The panel represents European and American origin potato cultivars with different market niches and phenotypic diversity for agronomic traits. Based on their market niche, the set includes 35 fresh consumption, 11 general purpose, 22 processing industry, 1 starch and 1 ancient cultivar. The materials were kindly provided by the Dutch potato breeding company HZPC Holland BV. 


\section{Phenotypic evaluation}

Ethiopian crop production is classified into two main seasons: a rainfed production season and irrigation production season. The rainfed production season is from May-October and is fully dependent on rainfall, while the irrigation production season is from October-April and fully dependent on irrigation water from rivers and streams. Phenotypic evaluation of the association mapping panel was carried out from May to September 2013 in the main production season (rainfed condition) at Debre-Tabor and Injibara and from February to June 2015 under irrigation production at Injibara and Koga in Ethiopia, following a split plot design with two replications at each location. As indicated in Chapter 2 and Chapter 3, the locations are found at different elevations with different rain fall, temperature and soil type. The low and high $\mathrm{N}$ levels (40 $\mathrm{kg} \mathrm{ha}^{-1}$ and $120 \mathrm{~kg} \mathrm{ha}^{-1}$, respectively) were assigned as main plots and the genotypes as sub-plots. The $\mathrm{N}$ fertilizer was applied using a side-banding method, half the amount applied a week after emergence and half at early flowering. Each experimental plot consisted of 10 plants, planted with inter- and intra-row spacing of $0.75 \mathrm{~m}$ and $0.30 \mathrm{~m}$ respectively and each plot was bordered by a reference cultivar.

The data were recorded on two to eight plants depending on the trait, selected randomly from the middle part of the rows. Days to emergence (DTE) was the number of days from planting till $50 \%$ of the plants in a plot emerged. Plant height (PH), Chlorophyll content (CC) at lower and upper part the leaves were measured using a SPAD-502 chlorophyll meter (Minolta Co., Ltd. Japan) when $50 \%$ of the genotypes were flowering. The readings for chlorophyll content were taken from the third or fourth leaf from the top of the plant for upper leaf chlorophyll content (UCC), and the second or third leaf from the base of the plant for lower leaf chlorophyll content (LCC). Stem number plant $^{-1}$ (SNPP) was the number of stems of a genotype counted before the plants collapsed. The canopy cover or soil cover (SC) was assessed every five days starting from date of emergence until the declining phase of the crop growth using a $0.6 \mathrm{~m} \mathrm{x}$ $0.75 \mathrm{~m}$ frame with 100 grid squares, positioned over the same middle plants in a plot for each measurement. Squares filled for more than $50 \%$ with foliage were counted, and the percentage of filled grid squares was considered canopy cover percentage.

Based on the canopy cover measurements, a curve was fitted using beta thermal time for each assessment, and model parameters describing the curve were estimated using the NOLIN procedure of SAS, SAS Institute Inc, 2004 (Khan et al., 2013). These include the inflection point in the build-up phase of the growth curve (tm1), time when the canopy cover reaches its maximum growth (t1), the maximum canopy cover (in percentage of soil cover) (Vmax), time of onset of canopy declining (t2), time when canopy cover reach to zero (te), Duration of max 
canopy( t2-t1), Duration of senescing of the canopy (te-t2), Area under the curve for growth phase one (emergence till t1) (AP1), Area under the curve for growth phase two (t1 till t2) (AP2), Area under the curve for growth phase three (t2 till te) (AP3), and area under the curve for the entire crop growth cycle (AUC) in \%.td. Days to maturity (DTM) was taken as the number of the days from emergence to the day at which more than $90 \%$ of the plants had changed their green foliage to yellow and attained physiological maturity. It was assessed every day starting from the time that early varieties showed the first signs of maturity (senescence of the foliage).

Tuber traits included tuber number plant $^{-1}$ (TNPP), average tuber weight (ATW), calculated as the ratio of the weight of tubers per plant and number of tubers per plant at harvest, tuber yield plant $^{-1}$ (TYPP), calculated as the tuber yield (fresh weight) of all harvested plants from a cultivar, divided by number of plants harvested. Specific gravity (SG), tuber dry matter percentage (TDM\%) and Nitrogen Use Efficiency (NUE) defined as dry tuber weight per unit $\mathrm{N}$ available were measured and calculated at harvest. To calculate the NUE, the dry tuber weight and $\mathrm{N}$ available was calculated in hectare base. Specific gravity (SG) was determined using the tuber specific gravity procedure of weight in air and under water (Murphy and Goven, 1959). Tuber dry matter percentage (TDM\%) normally is determined as a ratio of dry tuber weight to fresh weight expressed in percentage; we determined TDM\% indirectly from SG using empirical conversion factors following the equation of Kleinkopf et al. (1987): solid $($ Dry matter \% $)=-214.9206+(218.1852 \times \mathrm{SG})$.

\section{Statistical analyses}

\section{Phenotypic data analyses}

In two experimental seasons at three locations we have collected data for various agronomic and physiological traits of the association mapping panel. To estimate the variance components for each trait and assess the genotype-by-environment interaction, three types of analysis of variance (ANOVA) was performed:

1) Analysis of variance including all environments and $\mathrm{N}$ levels was carried out using the dataset from the three locations for each trait to get insight in the existence of genotypic variation and Genotype $\mathrm{x}$ Environment (Genotype $\mathrm{x}$ Location and Genotype $\mathrm{x} \mathrm{N}$ level) interaction, with Genstat version 18.1 edition. ANOVA was performed across locations using 
the general linear model for split plot design with two $\mathrm{N}$ level treatments as main plot and the genotypes as sub-plot, two replications and three locations.

2) Analysis of variance for low $\mathrm{N}$ and high $\mathrm{N}$ was done independently using all low $\mathrm{N}$ environment data, and all high $\mathrm{N}$ environment in separate analyses. Within each $\mathrm{N}$ level we had four environments (four low $\mathrm{N}$ and four high $\mathrm{N}$ environments). ANOVA was performed across four environments using the general linear model for randomized complete block design with four environments for each $\mathrm{N}$ level. This low $\mathrm{N}$ level and high $\mathrm{N}$ level location and production season combined data were used to find estimates of the genotypic variance $\left(\sigma^{2} \mathrm{~g}\right)$, Genotypeby- Environment interactions $\left(\sigma^{2}\right.$ ge) variance and environmental variance $\left(\sigma^{2}\right.$ e) for each trait at each $\mathrm{N}$ level. Subsequently, these estimates were used to calculate the heritability $\left(\mathrm{H}^{2}\right)$ of each trait based on genotype means over the four low $\mathrm{N}$ and four high $\mathrm{N}$ level environments using the formula: $H^{2}=\frac{\sigma^{2} g}{\sigma^{2} g+\left(\sigma^{2} g e / e\right)+\left(\sigma^{2} e / r e\right)}$ where e and $\mathrm{r}$ are the number of environments and replications per environment respectively. Combined mean values for low and high $\mathrm{N}$ were calculated for each trait by combining the phenotypic trait values for all four low $\mathrm{N}$ level environments and the four high $\mathrm{N}$ environments.

3) The third ANOVA was executed separately for each $\mathrm{N}$ level at each location, following a randomized complete block design system using one-way ANOVA. This data was used to estimate the genotypic variance $\left(\sigma^{2} \mathrm{~g}\right)$, environmental variance $\left(\sigma^{2} \mathrm{e}\right)$ for each trait and to calculate the heritability $\left(\mathrm{H}^{2}\right)$ of each trait based on genotype means at each location and at each $\mathrm{N}$ level using the formula: $H^{2}=\sigma^{2} g / \sigma^{2} g+\sigma^{2} e$.

The best linear unbiased estimates (BLUEs) were computed, to generate phenotypic values for marker trait association studies using breeding view, the IBP Breeding Management system (BMS) version 3.0.9 (https://www.integratedbreeding.net/breeding-management-systemBMSbreeding view software. Each $\mathrm{N}$ level at each location in each production season was considered as a single environment for the association mapping analysis with a total of eight environments.

\section{Genotypic data analyses}

The panel was genotyped with a 20k Infinium SNP array (Vos et al., 2015). 14,587 markers were scored in 5 dosage classes (nulliplex, simplex, duplex, triplex and tetraplex) depending on the number of copies of the allele (0 to 4) using fitTetra (Voorrips et al., 2011). Of the markers scored, 12,519 were polymorphic SNPs that were used for genome-wide association mapping. 
Linkage disequilibrium (LD) and population structure were calculated previously for a larger genotype set by d'Hoop et al. (2010) that included the 70 cultivars used for this study. All individual environments as well as combined mean data (all low $\mathrm{N}$ environments taken together and all high $\mathrm{N}$ environments) for each trait were subjected to single marker trait association analysis using the $\mathrm{R}$ software package for auto-tetraploids (GWASpoly). This package is unique in its ability to conduct the single marker test for association using different models of gene action (Rosyara et al., 2016). GWASpoly used mixed model analysis to perform marker trait association analysis, and for each trait four GWAS were conducted based on additive, simplex-dominant, duplex-dominant and general genetic models. The package used both a $\mathrm{Q}$ and a $\mathrm{K}$ matrix; the Q-matrix to account for population structure, and the K-matrix to correct for kinship of the association panel, to reduce the plausible but false marker-trait associations. Bonferroni correction for a genome wide-scan was used as a QTL detection threshold at 5\% significance level. When multiple significant markers were detected within a $10 \mathrm{Mb}$ region, only the most significant marker was reported along with the corresponding genetic model.

\section{Results}

\section{Phenotypic variation and heritability}

A summary of the analysis of variance for traits of the association panel at different $\mathrm{N}$ levels and locations is presented in supplementary Table 2. For the association panel, the analysis of variance that included all locations and $\mathrm{N}$ levels reveals that the variation due to genotypes and locations was highly significant for almost all of the traits measured. We used a T-test to check the significance of $\mathrm{N}$ level on traits measured in our experiment. Significant phenotypic variation $(\mathrm{P} \leq 0.001)$ was observed for $\mathrm{N}$ response in the association panel for most traits measured in this study except days to emergence (DTE), days to maturity (DTM), the inflection point in the build-up phase of the growth curve (tm1), time when the canopy cover reaches its maximum growth (t1), time for onset of canopy decline (t2), and time when canopy cover reaches zero (te). Various genotypes responded differently to locations as indicated by the highly significant $(\mathrm{P} \leq 0.01$ and 0.001$)$ interaction effect of genotype $(\mathrm{G}) \mathrm{x}$ location $(\mathrm{L})$ for most physiological and agronomic traits. However, the interaction of genotype $(\mathrm{G}) \times \mathrm{N}$ level $(\mathrm{N})$ was not significant for most traits, indicating G x L interaction had a larger contribution than $\mathrm{G} \times \mathrm{N}$ interaction to the total genotype-by-environment interaction (Supplementary Table 2).

The combined means over all locations, the minimum and maximum values of different agronomic and physiological traits in the association panel, and the variance components under low N (LN) and high N (HN) conditions are presented in Table 1. Significant reduction due to 
low N level was observed for Vmax and AUC (reduced by 26.22 and 29.2\%, respectively), and TNPP and TYPP (23.22 and $29.66 \%$ respectively). NUE increased more than 2 -fold under low $\mathrm{N}$ compared to high $\mathrm{N}$, suggesting that not all the available $\mathrm{N}$ under high $\mathrm{N}$ level conditions was effectively used for yield production.

The estimates of variance components under low and high $\mathrm{N}$ at each location and production system are presented in supplementary Table 3. For most traits, the environmental variance $\left(\sigma^{2} \mathrm{e}\right)$ was higher than the genotypic variance $\left(\sigma^{2} \mathrm{~g}\right)$ at both locations in each production system. However, the estimates of $\sigma^{2} \mathrm{~g}$ were higher than estimates of environments for traits such as $\mathrm{PH}$, LCC, UCC, SNPP, TNPP, TYPP, and ATW in the rainfed production system under low and high $\mathrm{N}$ at both locations. In the irrigation production system, most traits showed higher environmental variance than genotypic variance at both low and high $\mathrm{N}$.

The analysis of variance that included location and production system had estimates for genotype $\left(\sigma^{2} \mathrm{~g}\right)$ that were low compared to the estimates for environment $\left(\sigma^{2} \mathrm{e}\right)$ and genotypeby-environment interaction $\left(\sigma^{2}\right.$ ge), for most measured traits at both $\mathrm{N}$ levels (Table 1). This is reflected in the heritability estimates. Traits that showed a higher estimate of genotypic variance than environment at each location and production system also showed high genotypic variance in the analysis that compared production systems (with data from locations within a production system combined) (Supplementary Table 4), indicating that the effect of production season was large compared to location and $\mathrm{N}$ level. 
Table 1. Summary statistics of the association mapping panel for various agronomic and physiological trais (combined over location and production system) under low $\mathrm{N}$ (LN) and high $\mathrm{N}$ (HN) conditions

\begin{tabular}{|c|c|c|c|c|c|c|c|c|}
\hline \multirow[t]{2}{*}{ Traits } & \multirow[t]{2}{*}{ Treat } & \multirow[t]{2}{*}{ Mean } & \multicolumn{2}{|c|}{ Range } & \multicolumn{4}{|c|}{ Variance component } \\
\hline & & & Min & $\operatorname{Max}$ & $\sigma^{2} \mathrm{e}$ & $\sigma^{2} g$ & $\sigma^{2}$ ge & $\mathrm{H}^{2}$ \\
\hline \multirow[t]{2}{*}{ DTE } & $\mathrm{LN}$ & 24.0 & 20.0 & 28.0 & 9.6 & 1.01 & 2.08 & 0.4 \\
\hline & $\mathrm{HN}$ & 25.0 & 22.0 & 29.0 & 18.9 & 0.06 & 1.01 & 0.02 \\
\hline \multirow[t]{2}{*}{$\mathrm{PH}$} & $\mathrm{LN}$ & 31.0 & 25.0 & 39.0 & 16.3 & 5.7 & 14.3 & 0.5 \\
\hline & $\mathrm{HN}$ & 38.0 & 29.0 & 47.0 & 19.9 & 8.5 & 16.2 & 0.6 \\
\hline \multirow[t]{2}{*}{ SNPP } & $\mathrm{LN}$ & 3.0 & 2.0 & 5.0 & 0.5 & 0.2 & 0.2 & 0.6 \\
\hline & $\mathrm{HN}$ & 3.0 & 2.0 & 5.0 & 0.4 & 0.2 & 0.3 & 0.5 \\
\hline \multirow[t]{2}{*}{$\mathrm{LCC}$} & $\mathrm{LN}$ & 44.5 & 38.8 & 49.4 & 15.4 & 1.6 & 3.6 & 0.4 \\
\hline & $\mathrm{HN}$ & 47.3 & 42.7 & 52.8 & 12.8 & 1.2 & 7.8 & 0.3 \\
\hline \multirow[t]{2}{*}{ UCC } & $\mathrm{LN}$ & 42.4 & 37.1 & 47.1 & 10.04 & 0.6 & 3.6 & 0.2 \\
\hline & $\mathrm{HN}$ & 46.0 & 42.8 & 51.2 & 8.2 & 0.8 & 7.3 & 0.2 \\
\hline \multirow[t]{2}{*}{ DTM } & $\mathrm{LN}$ & 74.0 & 63.0 & 81.0 & 69.8 & 0.7 & 7.9 & 0.06 \\
\hline & $\mathrm{HN}$ & 73.0 & 65.0 & 80.0 & 65.3 & 1.2 & 14.4 & 0.09 \\
\hline \multirow[t]{2}{*}{ tm1 } & $\mathrm{LN}$ & 23.4 & 19.0 & 25.4 & 8.6 & 0.05 & -0.6 & 0.05 \\
\hline & $\mathrm{HN}$ & 22.4 & 19.1 & 25.0 & 12.8 & 0.2 & -1.4 & 0.16 \\
\hline \multirow[t]{2}{*}{$\mathrm{t} 1$} & LN & 30.4 & 27.5 & 32.1 & 7.9 & 0.07 & -0.3 & 0.07 \\
\hline & $\mathrm{HN}$ & 30.2 & 27.8 & 33.8 & 10.1 & 0.07 & 0.3 & 0.05 \\
\hline \multirow[t]{2}{*}{$\mathrm{t} 2$} & $\mathrm{LN}$ & 36.5 & 34.5 & 38.9 & 9.9 & 0.03 & 0.7 & 0.02 \\
\hline & $\mathrm{HN}$ & 36.2 & 33.2 & 39.1 & 8.2 & 0.01 & 2.3 & 0.01 \\
\hline \multirow[t]{2}{*}{$\mathrm{Te}$} & $\mathrm{LN}$ & 51.7 & 47.7 & 54.7 & 5.2 & 0.5 & 1.5 & 0.3 \\
\hline & $\mathrm{HN}$ & 50.5 & 48.1 & 52.5 & 5.9 & 0.2 & 0.6 & 0.2 \\
\hline \multirow[t]{2}{*}{$\mathrm{t} 2-\mathrm{t} 1$} & LN & 6.1 & 3.4 & 8.5 & 10.2 & 0.01 & 1.0 & 0.002 \\
\hline & $\mathrm{HN}$ & 6.0 & 3.8 & 9.7 & 10.6 & 0.00 & 1.2 & 0.001 \\
\hline \multirow[t]{2}{*}{ te-t 2} & $\mathrm{LN}$ & 15.3 & 12.3 & 18.4 & 15.3 & 0.6 & 0.6 & 0.2 \\
\hline & $\mathrm{HN}$ & 14.3 & 10.3 & 18.3 & 12.9 & 0.4 & 2.8 & 0.2 \\
\hline \multirow[t]{2}{*}{ Vmax } & $\mathrm{LN}$ & 45.4 & 36.5 & 58.6 & 90.5 & 7.06 & 31.4 & 0.3 \\
\hline & $\mathrm{HN}$ & 61.5 & 42.9 & 76.7 & 113.7 & 17.7 & 51.0 & 0.4 \\
\hline AP1 & $\mathrm{LN}$ & 404.0 & 259.4 & 531.6 & 20461.0 & 219.5 & 6126.0 & 0.05 \\
\hline
\end{tabular}




\begin{tabular}{|c|c|c|c|c|c|c|c|c|}
\hline \multirow[t]{2}{*}{ Traits } & \multirow[t]{2}{*}{ Treat } & \multirow[t]{2}{*}{ Mean } & \multicolumn{2}{|c|}{ Range } & \multicolumn{4}{|c|}{ Variance component } \\
\hline & & & Min & $\operatorname{Max}$ & $\sigma^{2} \mathrm{e}$ & $\sigma^{2} g$ & $\sigma^{2} \mathrm{ge}$ & $\mathrm{H}^{2}$ \\
\hline & $\mathrm{HN}$ & 572.8 & 409.9 & 795.1 & 31320.0 & $\begin{array}{l}101.0 \\
\end{array}$ & 10240.5 & 0.02 \\
\hline \multirow[t]{2}{*}{ AP2 } & $\mathrm{LN}$ & 255.8 & 145.3 & 406.8 & 21072.0 & 264.6 & 5230.0 & 0.06 \\
\hline & $\mathrm{HN}$ & 349.3 & 208.4 & 639.1 & 37189.0 & 1919.8 & 8780.0 & 0.2 \\
\hline \multirow[t]{2}{*}{ AP3 } & $\mathrm{LN}$ & 480.2 & 315.5 & 706.2 & 30944.0 & 1259.4 & 2315.0 & 0.2 \\
\hline & $\mathrm{HN}$ & 607.3 & 370.9 & 806.6 & 37029.0 & 1248.9 & 13926.5 & 0.13 \\
\hline \multirow[t]{2}{*}{ AUC } & $\mathrm{LN}$ & 1139.6 & 883.4 & 1575.6 & 58737.0 & 6313.9 & 32060.0 & 0.3 \\
\hline & $\mathrm{HN}$ & 1608.8 & 974.8 & 2174.6 & 113083.0 & 217184.1 & 3200749.5 & 0.06 \\
\hline \multirow[t]{2}{*}{ TNPP } & $\mathrm{LN}$ & 5.4 & 3.8 & 7.7 & 1.5 & 0.3 & 1.2 & 0.4 \\
\hline & $\mathrm{HN}$ & 7.0 & 5.4 & 10.2 & 3.8 & 0.5 & 0.6 & 0.4 \\
\hline \multirow[t]{2}{*}{ ATW } & $\mathrm{LN}$ & 74.8 & 44.8 & 103.9 & 314.9 & 57.3 & 201.2 & 0.4 \\
\hline & $\mathrm{HN}$ & 81.6 & 55.0 & 106.4 & 444.1 & 49.2 & 131.6 & 0.4 \\
\hline \multirow[t]{2}{*}{ TYPP } & $\mathrm{LN}$ & 380.2 & 269.5 & 558.3 & 10165.0 & 673.0 & 3193.0 & 0.3 \\
\hline & $\mathrm{HN}$ & 540.4 & 353.0 & 771.4 & 28994.0 & 1345.4 & 5128.0 & 0.2 \\
\hline \multirow[t]{2}{*}{ TDM\% } & $\mathrm{LN}$ & 13.2 & 9.6 & 16.9 & 13.9 & 0.6 & 1.6 & 0.2 \\
\hline & $\mathrm{HN}$ & 12.1 & 9.6 & 16.7 & 10.4 & 0.6 & 0.7 & 0.3 \\
\hline \multirow[t]{2}{*}{ NUE } & $\mathrm{LN}$ & 59.4 & 36.0 & 98.4 & 816.4 & 19.3 & -56.0 & 0.2 \\
\hline & $\mathrm{HN}$ & 27.0 & 16.6 & 38.9 & 196.2 & 3.1 & -14.2 & 0.13 \\
\hline
\end{tabular}

DTE $=$ Days to emergence, $\mathrm{PH}=$ Plant height in $\mathrm{cm}, \mathrm{SNPP}=$ Stem number plant ${ }^{-1}$, LCC $=$ Lower leaf chlorophyll content, UCC $=$ Upper leaf chlorophyll content, DTM = Days to maturity, $\mathrm{tml}=$ inflection point in the build-up phase of the growth curve in thermal day (td), $\mathrm{t} 1=$ time when the canopy cover reaches its maximum growth in $\mathrm{td}, \mathrm{t} 2=$ time of onset of canopy declining in td, te $=$ time when canopy cover reach to zero in td, $\mathrm{t} 2-\mathrm{t} 1=$ Duration of max canopy in $\mathrm{td}$, te-t $2=$ Duration of senescing of the canopy in td, Vmax = the maximum canopy cover in \%, AP1= Area for growth phase one (emergence till t1) in \% td, AP2 = Area for growth phase two (t1 till $\mathrm{t} 2$ ) in $\% \mathrm{td}, \mathrm{AP} 3=$ Area for growth phase three (t2 till te) in $\% \mathrm{td}, \mathrm{AUC}=$ area under the curve for the entire crop growth cycle in $\%$ td, TNPP $=$ Tuber number plant $^{-1}$, ATW $=$ Average tuber weight in $\mathrm{g}$, TYPP $=$ Tuber yield plant $^{-1}$ in $\mathrm{g}$, TDM $\%=$ Tuber dry matter in percent, NUE $=$ Nitrogen use efficiency $\left(\mathrm{kg} \mathrm{kg}^{-1}\right), \mathrm{LN}=$ low $\mathrm{N}\left(40 \mathrm{~kg} \mathrm{ha}^{-1}\right), \mathrm{HN}=$ high $\mathrm{N}\left(120 \mathrm{~kg} \mathrm{ha}^{-1}\right) . \sigma \mathrm{e} 2=$ environmental variance, $\sigma \mathrm{g}^{2}=$ genotypic variance,$\sigma g e 2=$ genotype $\mathrm{x}$ environement interaction variance, $\mathrm{H}^{2}=$ broad sense heritability

The association panel included three distinct market groups (fresh consumption type, general purpose, and processing type, consisting of 35, 12 and 23 cultivars respectively). Although not statistically significant, considerable differences in TDM\% and NUE were observed between the market groups under low and high $\mathrm{N}$ conditions. In the rainfed production system, the processing group scored considerably higher than the fresh consumption and general purpose 
group for both TDM\% and NUE especially under low N conditions (Fig. 1a, c). The effect of N levels on TDM\% and NUE was also most clear in the rainfed production system. In the irrigation production system, a clear difference was only observed between $\mathrm{N}$ levels for NUE (Fig 1b, d) and not between marketing groups.
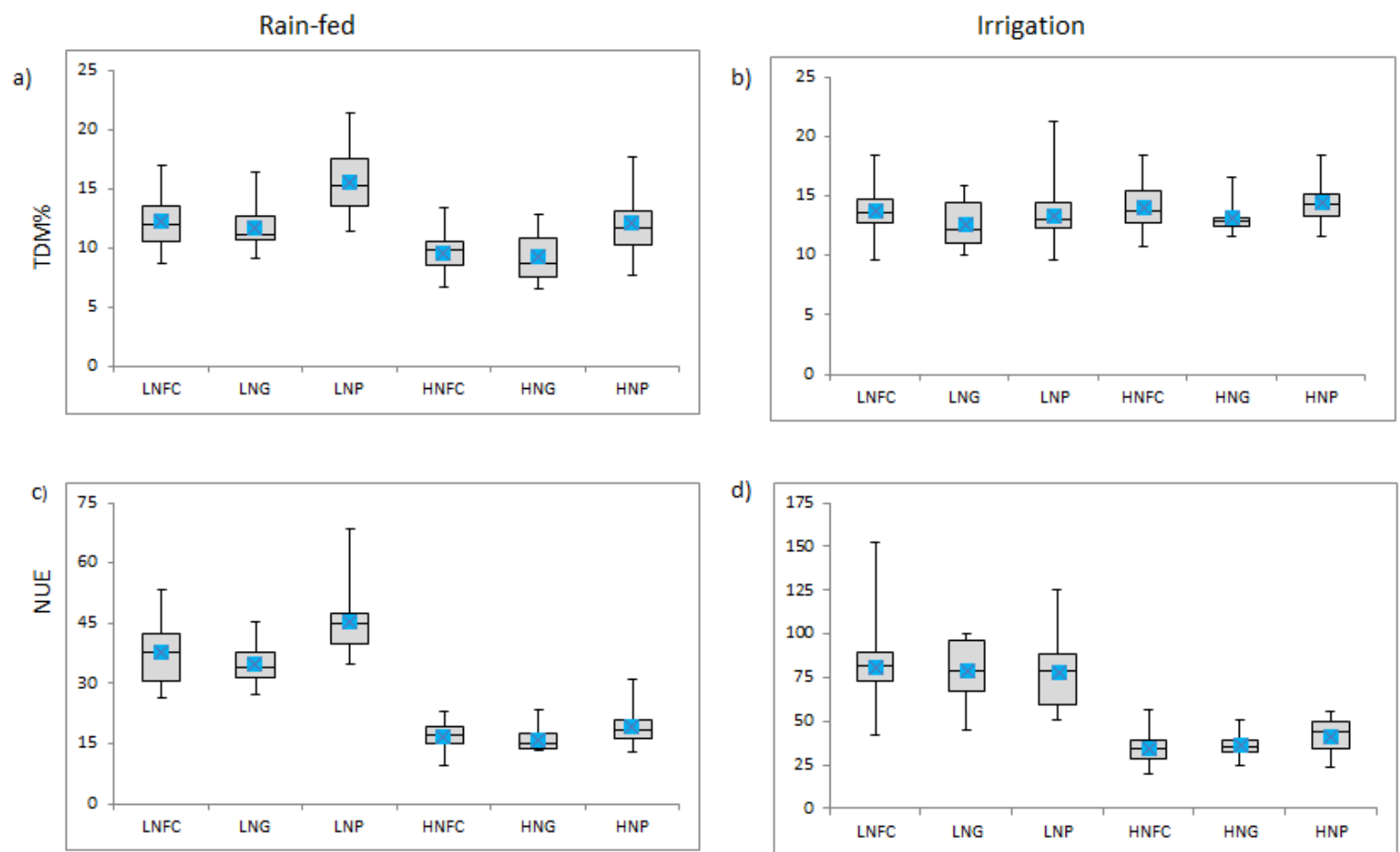

Figure 1. Box plots of selected traits of cultivars separated in market niche groups under different $\mathrm{N}$ levels in rainfed irrigation production systems. $\mathrm{LNFC}=$ low nitrogen fresh consumption, $\mathrm{LNG}=$ low nitrogen general purpose, $\mathrm{LNP}=$ low nitrogen processing, $\mathrm{HNFC}=$ high nitrogen fresh consumption, $\mathrm{HNG}=$ high nitrogen general purpose, $\mathrm{HNP}=$ high nitrogen processing type. TDM\% = tuber dry matter in \%, NUE = nitrogen use efficiency $\left(\mathrm{kg} \mathrm{kg}^{-1}\right)$

Overall, higher tuber yield and NUE values were recorded under irrigation compared to rainfed production season. This may be related to the amount and distribution of rain in the rainfed season. The rain fall was heavy and frequent from mid-June to August (growing and bulking period of potato), and this high rain fall favoured potato diseases and insect pest occurrence, which may have reduced the tuber yield and NUE. In fact, we applied fungicide (redomil) for the management of late blight on the foliage before infection or when the disease is in very early stages. However, confounding disease symptoms were observed in addition to late blight that may have reduced the performance of the cultivars. Heavy and frequent rain may have also reduced available $\mathrm{N}$ in the rainfed locations compared to the irrigated locations. 
The Pearson correlation coefficients (r) between various physiological and agronomic traits under low $\mathrm{N}$ and high $\mathrm{N}$ conditions are presented in Table 2. NUE showed strong positive correlations with Vmax, AP3, AUC, TNPP, TYPP and TDM\% under both N levels indicating the potential of these traits for indirect selection for NUE under low and high $\mathrm{N}$ level conditions. Most traits considered in this study showed medium to high positive correlations between low and high $\mathrm{N}$ conditions. 
Table 2. Correlation coefficients of the four-environment combined agronomic and physiological traits of the association mapping panel under high $\mathrm{N}$ (above diagonal) and low $\mathrm{N}$ (below diagonal)

\begin{tabular}{|c|c|c|c|c|c|c|c|c|c|c|c|c|c|c|c|c|c|c|c|}
\hline \multirow{2}{*}{$\begin{array}{l}\text { Low } \\
\mathrm{N}\end{array}$} & \multirow{2}{*}{$\begin{array}{l}\text { High N } \\
\text { DTE }\end{array}$} & \multirow{2}{*}{$\begin{array}{l}\text { DTE } \\
\mathbf{0 . 3 5}\end{array}$} & \multirow{2}{*}{$\begin{array}{l}\text { SNPP } \\
-0.26\end{array}$} & \multirow{2}{*}{$\begin{array}{l}\text { UCC } \\
0.23\end{array}$} & \multirow{2}{*}{$\begin{array}{l}\mathrm{tm} 1 \\
0.09\end{array}$} & \multirow{2}{*}{$\begin{array}{l}\mathrm{t} 1 \\
-0.13\end{array}$} & \multirow{2}{*}{$\begin{array}{l}\mathrm{t} 2 \\
-0.14\end{array}$} & \multirow{2}{*}{$\begin{array}{l}\text { te } \\
-0.11\end{array}$} & \multirow{2}{*}{$\begin{array}{l}\text { Vmax } \\
0.13\end{array}$} & \multirow{2}{*}{$\begin{array}{l}\mathrm{t} 2-\mathrm{t} 1 \\
-0.02\end{array}$} & \multirow{2}{*}{$\begin{array}{l}\text { te-t } 2 \\
0.03\end{array}$} & \multirow{2}{*}{$\begin{array}{l}\text { AP2 } \\
0.12\end{array}$} & \multirow{2}{*}{$\begin{array}{l}\text { AP3 } \\
0.13\end{array}$} & \multirow{2}{*}{$\begin{array}{l}\text { AUC } \\
0.15\end{array}$} & \multirow{2}{*}{$\begin{array}{l}\text { DTM } \\
-0.17\end{array}$} & \multirow{2}{*}{$\begin{array}{l}\text { TYPP } \\
0.12\end{array}$} & \multirow{2}{*}{$\begin{array}{l}\text { TNPP } \\
-0.05\end{array}$} & \multirow{2}{*}{$\begin{array}{l}\text { TDM\% } \\
0.02\end{array}$} & \multirow{2}{*}{$\begin{array}{l}\text { NUE } \\
0.1\end{array}$} \\
\hline & & & & & & & & & & & & & & & & & & & \\
\hline & SNPP & -0.41 & 0.74 & -0.23 & -0.14 & 0.02 & 0.07 & 0.06 & 0.1 & 0.05 & -0.01 & 0.08 & 0.03 & 0.04 & 0.08 & 0.03 & 0.41 & 0.07 & 0.03 \\
\hline & UCC & -0.15 & 0.01 & 0.46 & 0.23 & -0.01 & 0.11 & 0.09 & -0.16 & 0.12 & -0.02 & -0.02 & -0.09 & -0.1 & 0.28 & -0.15 & -0.23 & -0.08 & -0.17 \\
\hline & $\mathrm{tm} 1$ & -0.23 & 0.09 & 0.08 & 0.00 & 0.55 & 0.21 & 0.08 & 0.21 & -0.31 & -0.11 & -0.11 & 0.12 & 0.09 & 0.2 & 0.11 & -0.06 & 0.01 & 0.14 \\
\hline & $\mathrm{t} 1$ & -0.3 & 0.27 & 0.02 & 0.46 & 0.14 & 0.47 & 0.21 & 0.06 & -0.48 & -0.22 & -0.33 & -0.05 & 0.04 & 0.24 & -0.18 & -0.01 & 0.01 & -0.15 \\
\hline & $\mathrm{t} 2$ & 0.06 & -0.05 & 0.04 & 0.36 & 0.39 & 0.36 & -0.01 & 0.25 & 0.55 & -0.76 & 0.54 & -0.32 & 0.3 & 0.19 & 0.04 & 0.22 & 0.21 & 0.12 \\
\hline & te & -0.36 & 0.23 & 0.11 & 0.62 & 0.42 & 0.06 & 0.49 & 0.35 & -0.2 & 0.66 & -0.08 & 0.7 & 0.37 & 0.44 & 0.31 & 0.14 & 0.03 & 0.23 \\
\hline & $V \max$ & 0.18 & -0.02 & -0.22 & 0.22 & 0.22 & 0.26 & 0.15 & 0.59 & 0.19 & 0.05 & 0.6 & 0.72 & 0.93 & 0.23 & 0.54 & 0.53 & 0.27 & 0.53 \\
\hline & $\mathrm{t} 2-\mathrm{t} 1$ & 0.33 & -0.29 & 0.01 & -0.09 & -0.55 & 0.55 & -0.33 & 0.03 & 0.32 & -0.54 & 0.85 & -0.27 & 0.27 & -0.04 & 0.22 & 0.23 & 0.21 & 0.26 \\
\hline & te-t 2 & -0.33 & 0.21 & 0.06 & 0.26 & 0.08 & -0.6 & 0.76 & -0.04 & -0.62 & 0.44 & -0.46 & 0.7 & 0.01 & 0.15 & 0.17 & -0.07 & -0.14 & 0.06 \\
\hline & AP2 & 0.39 & -0.28 & -0.13 & 0.1 & -0.3 & 0.57 & -0.15 & 0.55 & 0.79 & -0.49 & 0.57 & 0.08 & 0.65 & 0.03 & 0.37 & 0.43 & 0.34 & 0.44 \\
\hline & AP3 & -0.09 & 0.12 & -0.07 & 0.32 & 0.22 & -0.19 & 0.61 & 0.74 & -0.38 & 0.61 & 0.08 & 0.5 & 0.68 & 0.26 & 0.48 & 0.28 & 0.06 & 0.39 \\
\hline & AUC & 0.16 & -0.01 & -0.14 & 0.15 & 0.21 & 0.26 & 0.24 & 0.96 & 0.05 & 0.02 & 0.57 & 0.77 & 0.72 & 0.2 & 0.46 & 0.48 & 0.3 & 0.49 \\
\hline & DTM & -0.52 & 0.08 & 0.22 & 0.37 & 0.32 & 0.09 & 0.5 & 0.15 & -0.21 & 0.34 & -0.09 & 0.35 & 0.2 & 0.16 & 0.16 & -0.05 & -0.06 & 0.05 \\
\hline & TYPP & 0.09 & -0.05 & 0.12 & 0.13 & 0.11 & 0.29 & 0.26 & 0.6 & 0.16 & 0.02 & 0.39 & 0.52 & 0.62 & 0.18 & 0.53 & 0.38 & -0.09 & 0.78 \\
\hline & TNPP & -0.14 & 0.38 & 0 & 0.2 & 0.21 & 0.08 & 0.25 & 0.3 & -0.12 & 0.15 & 0.03 & 0.34 & 0.27 & 0.11 & 0.36 & 0.65 & 0.29 & 0.46 \\
\hline & TDM\% & -0.1 & 0.15 & -0.16 & 0.09 & 0.09 & -0.01 & 0.1 & 0.32 & -0.09 & 0.09 & 0.14 & 0.28 & 0.3 & 0.07 & -0.03 & 0.18 & 0.52 & 0.48 \\
\hline & NUE & -0.05 & 0.11 & 0.07 & 0.2 & 0.21 & 0.2 & 0.31 & 0.57 & -0.01 & 0.12 & 0.27 & 0.54 & 0.58 & 0.25 & 0.67 & 0.39 & 0.64 & 0.32 \\
\hline
\end{tabular}

color key

$$
-1 \quad 0
$$

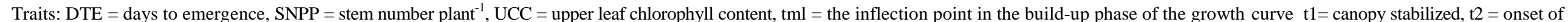

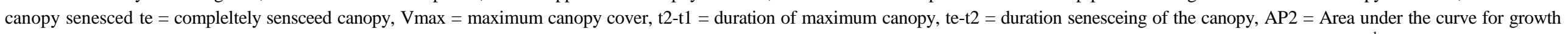

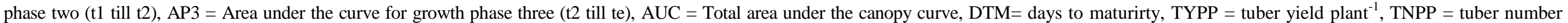
plant $-{ }^{1}$, TDM $\%=$ tuber dry matter $\%$, NUE = nitrogen use effeciency 


\section{Association mapping}

Association mapping was performed with 12,519 polymorphic SNP markers, using a Q + K matrix to correct for population structure and kinship in the association panel. We defined the marker-trait associations (MTA) that were within a $10 \mathrm{Mb}$ region as a single QTL, and only report the peak marker in this region. QTLs above the calculated threshold of $\mathrm{p} \leq 0.05$ ($\log (10) \mathrm{P}$ value of 4.5) for NUE and NUE-related agronomic and physiological traits measured in 15 environments including the over-environment combined data, with allele frequencies above 5\%, are presented in Supplementary Table 2. The significant MTAs in these QTLs had $\log 10(\mathrm{P})$ values ranging from 4.52 to 7.28 . Of the identified QTL regions, 18 harbour QTLs for two or more traits (Table 3). In total 77 QTLs were detected for 18 measured or calculated phenotypic traits in 8 low and high $\mathrm{N}$ single environments, and production season, low $\mathrm{N}$, and high N combined environments (Supplementary Table 5).

QTLs for NUE and other agronomic and physiological traits that have a strong positive correlation with NUE were detected on chromosomes, III, V and VI under various environments. On chromosome III, QTLs for NUE and AUC co-localized at the same genomic region in low $\mathrm{N}$ environments, while QTLs for $\mathrm{t} 2$, $\mathrm{t} 2-\mathrm{t} 1$, DTM and TDM\% under different environments were clustered on another region of the same chromosome. SNPP, tm1, t2, t2-t1, te, Vmax, AUC, TDM\% and NUE had QTLs on chromosome V, with QTLs for Vmax, AUC, and TDM\% co-localising in the same region with NUE between 351,772bp and 9,824,216bp. A region between 52,929,083bp and 58,844,975bp on chromosome VI harboured QTLs for NUE and other NUE related traits such as TNPP, UCC and AP2.

AUC and DTM shared an association with the same marker (PotVar0010985) on chromosome IX under high $\mathrm{N}$ conditions in the rainfed production season at Injibara. Other QTLs for AUC were detected on chromosome III, V, and IX, and marker PotVar0019456 on chromosome III was associated with AUC in over location and production season combined low $\mathrm{N}$ environment and irrigation production season combined low N environment. This QTL can be considered as a low $\mathrm{N}$ dependent but location and production season-independent QTL for AUC. QTLs were detected on chromosomes I, IV, XI and XII for TYPP, and particularly QTLs linked with the marker solcap_snp_c2_26796 on chromosome IV and marker PotVar0060022 on chromosome XI were associated with TYPP in two low $\mathrm{N}$ condition environments under irrigation production system, indicating that these QTLs were low $\mathrm{N}$ as well as irrigation season dependent. QTLs detected for traits t1, Vmax, AP3, DTM and NUE were specific for rainfed production season, while TYPP and te-t2 were irrigation production season specific QTLs. 
SNPP, tm1, t2-t1, AP2, TNPP and TYPP had QTLs in more than one environment under both production systems. Constitutive QTLs were detected for the major tuber yield component trait TNPP on chromosome VII with marker solcap_snp_c2_25261 and for SNPP on chromosome $\mathrm{X}$ with marker PotVar0116800 in different environments.

We detected more than one QTL for most traits in several environments, but environmentspecific or environment-excluding QTLs were also identified, suggesting presence of QTL $\mathrm{x}$ environment interaction. A summary of QTLs in various environments for each trait is presented in Supplementary Table 2. Only 8\% of these QTLs were detected in two or more environments and the remaining 92\% were environment-specific. Of the total identified QTLs in both single or combined low and high $\mathrm{N}$ environments, $49 \%$ were detected in high $\mathrm{N}$ level environments, $46 \%$ in low $\mathrm{N}$ level environments, and $5 \%$ in combined environments. From the detected QTLs 38 (49\%) were detected only in rainfed and $21(27 \%)$ only in irrigation production systems. Overall, most QTLs identified in this study were environment-dependent indicating presence of QTL-by-Environment interaction in our association panel. 
Table 3. list of 18 QTL regions (within $10 \mathrm{Mb}$ ) that harbour QTLs for two or more traits

\begin{tabular}{|c|c|c|}
\hline Chromosome & Traits & Chromosome region(bp ) \\
\hline \multirow[t]{2}{*}{ I } & t2 \& TYPP & $51,293,720-58,197,448$ \\
\hline & SNPP, DTE \& AP2 & $75,179,906-82,029,061$ \\
\hline II & TNPP, t1, t2-t1 \& SNPP & $50,890,271-55,659,310$ \\
\hline \multirow[t]{2}{*}{ III } & NUE \& AUC & $2,235,688-2,742,393$ \\
\hline & $\mathrm{TDM} \%, \mathrm{t} 2-\mathrm{t} 1, \mathrm{DTM} \& \mathrm{t} 2$ & $50,890,271-55,659,310$ \\
\hline \multirow[t]{2}{*}{ IV } & DTM, t2-t1 \& AP3 & $66,147,280-67,807,068$ \\
\hline & TYPP \& te & $641,790-9,524,541$ \\
\hline \multirow[t]{3}{*}{$\mathrm{V}$} & $\mathrm{tm} 1, \mathrm{NUE}, \mathrm{SNPP}, \mathrm{TDM} \%$ \& $\mathrm{t} 2-\mathrm{t} 1$ & $2,964,094-12,658,442$ \\
\hline & t2 \& te & $50,863,328-51,682,609$ \\
\hline & Vmax \& AUC & $351,772-1,413,732$ \\
\hline VI & NUE, TNPP, UCC \& AP2 & $52,929,083-58,844,975$ \\
\hline VII & te-t2, AP2, TNPP \& t2-t1 & $44,288,221-50,155,639$ \\
\hline VIII & SNPP \&TDM $\%$ & $53,835,553-56,624,935$ \\
\hline \multirow[t]{2}{*}{ IX } & te, UCC, AUC \& DTM & $52,407,969-57,422,879$ \\
\hline & $\mathrm{AP} 2 \& \mathrm{tm} 1$ & $48,735,918-49,977,704$ \\
\hline $\mathrm{x}$ & SNPP & $1,910,636$ \\
\hline XI & TNPP \& tm1 & $40,967,802-44,430,446$ \\
\hline XII & TNPP \& TYPP & $59,793,350-59,957,211$ \\
\hline
\end{tabular}

Traits: $\mathrm{t} 2=$ onset of canopy senesced, TYPP $=$ tuber yield per plant, $\mathrm{SNPP}=$ stem number plant $^{-1}, \mathrm{DTE}=$ days to emergence, $\mathrm{AP} 2=\mathrm{Area}$ under the curve for growth phase two ( $\mathrm{t} 1$ till $\mathrm{t} 2$ ), TNPP $=$ tuber number plant ${ }^{-1}, \mathrm{t} 1=$ canopy stabilized, $\mathrm{t} 2-\mathrm{t} 1=$ duration of maximum canopy, NUE $=$ nitrogen use efficiency, AUC $=$ Total area under the curve, $\mathrm{TDM} \%=$ tuber dry matter\%, DTM= days to maturirty, AP3 = Area under the curve for growth phase three(t2 till te), te $=$ compleltely sensceed canopy, $\mathrm{tml}=$ the inflection point in the build-up phase of the growth curve Vmax = maximum canopy cover, $\mathrm{UCC}=$ upper leaf chlorophyll content, te-t $2=$ duration senesceing of the canopy

To detect MTAs and QTLs, various gene models were used in separate association analyses. In this study, a simplex-dominant genetic model, duplex-dominant genetic model, additive and general genetic models were used to identify MTAs, and the majority (75\%) of the MTAs were identified using dominant genetic models. All MTAs identified for NUE, AUC and t1 were using dominant genetic models, while other traits had MTAs detected by general, dominant and additive genetic models (see also Figure 3). The quantile-quantile (QQ) plots (Figure 3b, d, f and $\mathrm{h}$ ) demonstrate that the $\mathrm{Q}+\mathrm{K}$ mixed model allowed as to reduce the false marker trait associations, as quantified by the linear regression coefficient of the observed vs expected $\log (10) \mathrm{P}$ values. 
a)

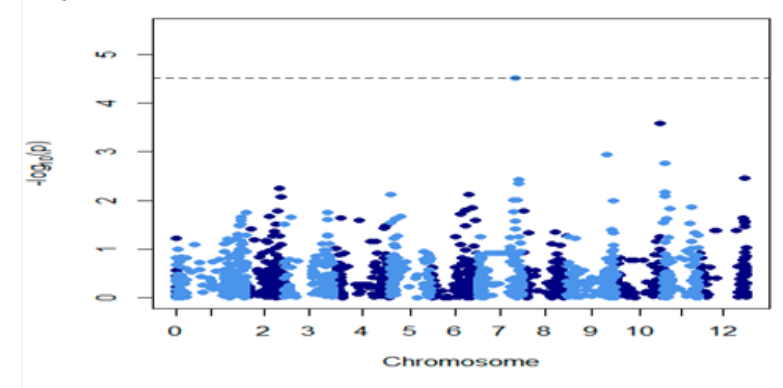

c)

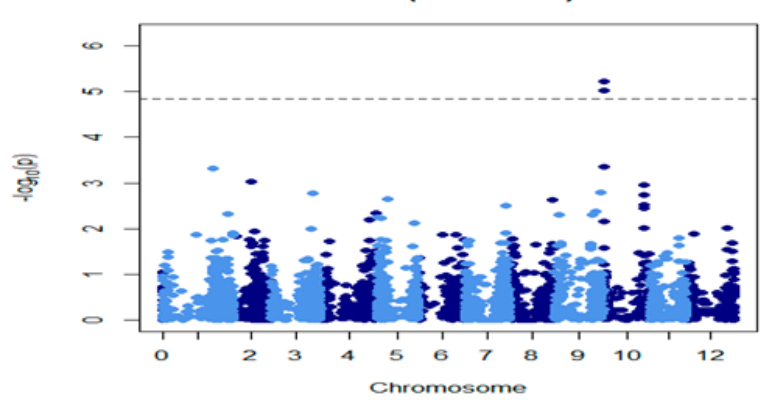

e)

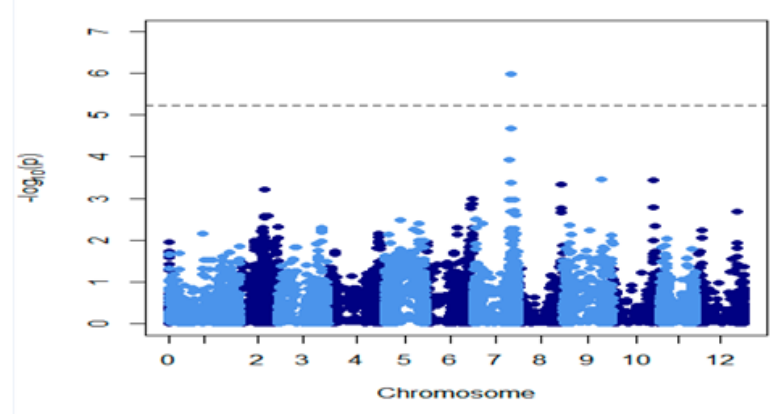

g)

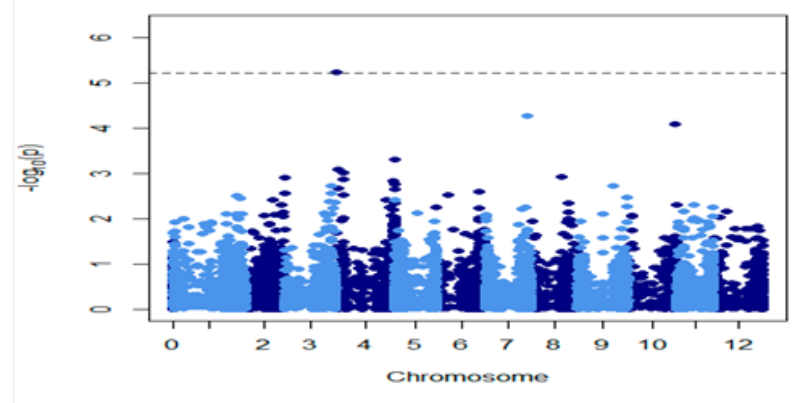

b)

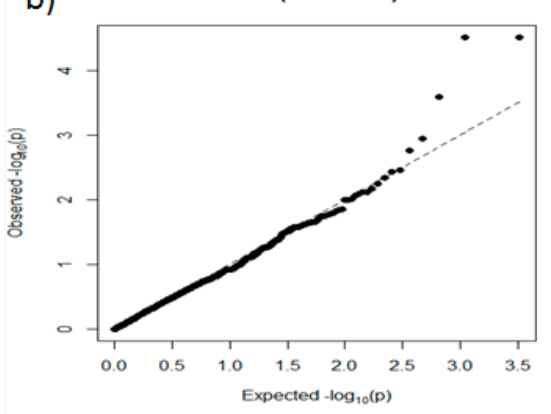

d)

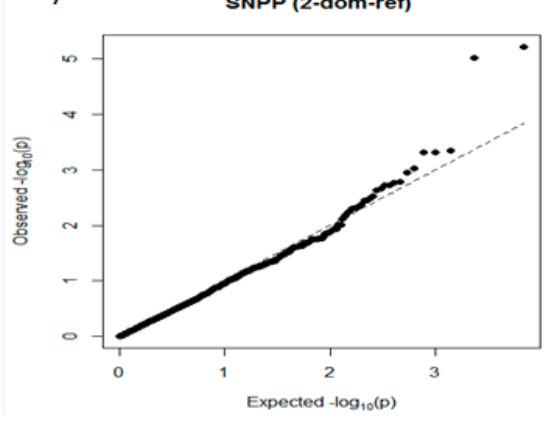

f)

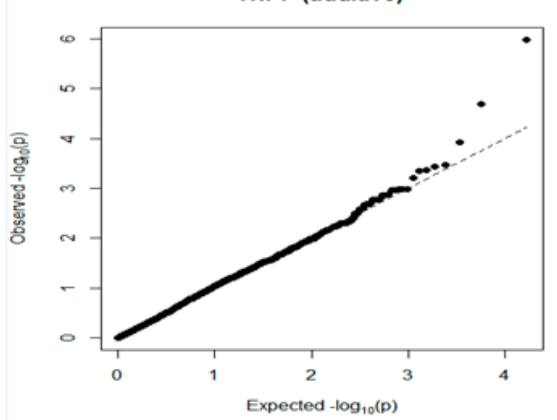

h)

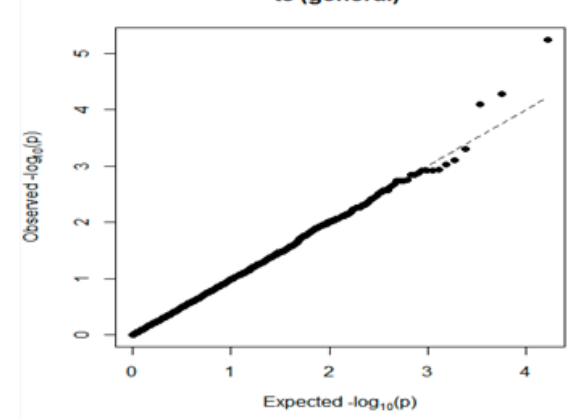

Figure 3. Manhattan plots and QQ plots for several traits using different gene models. (a) Manhattan plot of the simplex dominant model for area under the canopy curve for maximum canopy cover phase (AP2). The $-\log 10$ P-values from genome wide scan are plotted against the positions of the 12 chromosomes. The horizontal dotted line indicates the significance threshold ( $\mathrm{P} \leq 0.05)$. (b) Quantile-quantile (QQ) plot of the simplex dominant model for AP2. (c) Manhattan plot of the duplex dominant model for stem number plant ${ }^{-1}$ (SNPP). (d) Quantile-quantile plot of duplex dominant model for SNPP. (e) Manhattan plot of additive model for tuber number plant ${ }^{-1}$ (TNPP). (f) Quantile-quantile plot of additive model for TNPP. (g) Manhattan plot of general model for complete canopy senesced (te). (h) Quantile-quantile plot of the general model for te. 


\section{Discussion}

\section{Phenotypic variation}

NUE is a complex physiological process determined in potato by the efficient uptake, accumulation and partitioning of nitrogen and assimilates to facilitate production of tubers. Uncovering the mechanisms underlying such a complex trait is not straightforward due to the complexity of the adaptive response of the crops to changes in available N. In this study, we present the genetic analysis of NUE in European commercial potato cultivars, linking traits that contribute to NUE under low and high $\mathrm{N}$ conditions to specific genomic regions in potato. The cultivars were evaluated under low and high $\mathrm{N}$ conditions in rainfed and irrigation production systems in different locations and years, and the genotype-by-environment interaction was significant for most traits considered in this study. The environment-combined variance component analysis showed low genotypic variance $\left(\sigma^{2} \mathrm{~g}\right)$ compared to estimates of genotypeby-environment interaction variance $\left(\sigma^{2}\right.$ ge) and environmental variance $\left(\sigma^{2}\right.$ e) for all traits, indicating presence of large differences between environments. In particular, the genotype-bylocation interaction, which includes production season, had larger contributions to the total genotype-by-environment interaction than the genotype-by- $\mathrm{N}$ level interaction, indicating a significant effect of experimental locations as well as production seasons on the performance of potato cultivars. In most potato growing countries potato cultivars grown in specific areas are selected according to the environmental conditions prevailing in these countries. Lisinska and Leszczynski (1989) reported that tuber yield and quality traits of potato were generally linked to the prevalent climatic conditions of a given area. In our study, the large contribution of genotype-by-location interaction to total genotype-by environment interaction could at least partly be attributed to differences in production season (rainfed vs irrigated), and these may be the predominant non-genetic factors affecting growth, yield and NUE of potato. This high environmental variance compared to genotypic variance particularly in the irrigation production system may be attributed to irrigation management. As we conducted the field experiment using furrow irrigation method, seepage and other factors may enhance within field variation, and thus increase the environmental variance in the irrigation experiments.

NUE and most-NUE related traits had high genotypic variance and heritability estimates under rainfed production season conditions, whereas under irrigation the estimates were low for most traits (Supplementary table 5). As shown in Supplementary Table 4, the $\mathrm{H}^{2}$ estimate differences between locations and $\mathrm{N}$ levels within each production season were small for most traits, while the $\mathrm{H}^{2}$ estimate differences between production seasons (rainfed vs irrigation) in the locations 
combined data was large for most agronomic and physiological traits (Supplementary Table 5). This further points to production season contributing more to the total genotype-byenvironment interaction than locations and $\mathrm{N}$ levels. Thus, our study suggests that the target breeding environment should be divided into sub-target environments (mega-environments) based on production seasons, so as to increase the heritability and selection efficiency of potato for NUE (for a more detailed study of genotype-by-environment interaction see Chapter 5).

Within the market groups in our potato genotype set, the processing type group had higher values of DM\% and NUE compared to the other two groups especially under rainfed conditions. Bártá and Bártová (2008) determined extractable protein in European processing and table potato cultivars and reported that tuber dry matter percentage was significantly higher in processing potato cultivars than table potato cultivars, in line with our results under rainfed conditions. Remarkably, this difference was not observed under irrigated conditions. Whether this difference is related to a season influence remains to be established.

\section{Genetic variation for NUE in potato}

The phenotypic data analysis showed that there is genetic variation in the panel population for traits related to NUE, indicating that the population may be a suitable panel for detection of QTLs for these traits, provided that the population structure and linkage disequilibrium (LD) are acceptable. Population structure (unaccounted sub-populations in the genotype set) induces LD between unlinked loci (Pritchard et al., 2000). Consequently, some marker-trait associations that are statistically associated to the analysed trait may not be genetically associated with the phenotypic variation (Mezmouk et al., 2011). We used Q and K matrixes to correct for the population structure and kinship, moreover linkage disequilibrium (LD) and population structure were calculated previously for a larger genotype set by d'Hoop et al. (2010) that included the 70 cultivars used for this study. Both results revealed that there was no clear structure in the panel, suggesting that it is suitable for association mapping studies. In the identification of significant MTAs, we tried to reduce false positives. As showed in the quantile-quantile plot, inflation of the P-value above the linear regression line was very low, which is an indication that the models successfully control the population structure. Moreover, rare alleles were removed and the MTAs were identified using Bonferroni correction as a threshold test, which is one of the most conservative approaches to avoid spurious positives (Rosyara et al., 2016). All the aforementioned tests make it likely that the identified MTAs are indeed resulting from genetic linkage of the markers to the phenotypic traits. 
The cultivars in the association panel were mainly developed for high $\mathrm{N}$ input and European long day conditions, and they may lack genetic diversity and optimal alleles for NUE and related traits under low $\mathrm{N}$ conditions and short days, which is the most common cultivation environment in Ethiopia. Many authors indeed reported that modern plant breeding may have reduced crop genetic diversity (Borlaug, 2007; Govindaraj et al., 2015; Babiker et al., 2015), and this may be especially true for traits that were not selected for. However, in our panel there was still considerable variation for NUE and related traits under both low and high $\mathrm{N}$ conditions. This variation may be attributed to the fact that it includes cultivars released for production over a wide range of years and for different purposes. Based on the frequency of genotypes in the markers scored, the processing cultivars contributed more positive alleles to the identified NUE QTLs under low $\mathrm{N}$ level condition, while under high $\mathrm{N}$ fresh consumption type cultivars contributed more compared to processing types. Of the 8 cultivars which have more positive allele contribution for the identified QTLs under low N 5 cultivars were processing type. Under high N, 7 cultivars showed higher contribution of positive alleles of which 4 were fresh consumption type. This suggests that the two groups have $\mathrm{N}$ level-specific genetic potential for NUE improvement of potato.

The QTL mapping described in Chapter 3 using the CxE diploid backcross population conducted in a similar field experimental set-up resulted in the detection of several QTL regions. Many QTLs were detected on chromosome V, with QTLs controlling NUE and other physiological and morphological traits accumulating at different genomic locations. QTLs found with a bi-parental mapping approach often cannot directly lead to identification of candidate genes, mainly due to the often-low resolution of biparental QTL mapping (Bernardo, 2008). Association mapping typically has a higher resolution compared to QTL mapping due to the higher number of recombination and it does not confound the analysis of non-additive gene effect like dominant gene effect. In our association mapping approach under low and high $\mathrm{N}$ conditions we identified QTLs in different regions of the potato genome, including several associations accumulating on chromosome V (Supplementary Table 2). Markers associated with Vmax and AUC co-located between 351,772bp and 1,413,732bp on chromosome V. The SNP marker PotVar0026355 on chromosome V, positioned at 4,335,324bp, associated with NUE in the present study and is $3.83 \mathrm{Mb}$ away from the PotSNP573 marker positioned at 507,660bp which was associated with NUE in the CxE QTL mapping study (this thesis Chapter 3). The marker PotVar0026355 is also about $0.34 \mathrm{Mb}$ away from the SSR marker Mando located at $4.67 \mathrm{Mb}$, which was associated with days to maturity in the same linkage mapping 
study (Chapter 3) and 0.16Mb from the CDF1 gene (PGSC0003DMG400018408) shown to be responsible for early maturity and involved in photoperiod-induced initiation of tuberisation (Kloosterman et al., 2013). The co-localization of these QTLs in both a biparental and an association mapping population validates the detected QTLs. The presence of QTLs for NUE and days to maturity in close proximity to the CDF1 gene suggests that this gene underlies both maturity and NUE, and that NUE and maturity are correlated. Indeed Tiemens-Hulscher et al. (2012) reported that differences in NUE under high and low N input conditions were shown to be strongly associated with maturity type. Others reported that late maturing potato cultivars recorded higher NUE values than early maturing ones under both high and low $\mathrm{N}$ conditions (Zebarth et al., 2004, Ospina et al., 2014), which was confirmed in our genetic diversity study in Chapter 2. In the current study, the environment-combined correlation between NUE and days to maturity (DTM) was low (Table 2); we found a high correlation between the two traits in the rainfed production season (Chapter 2), while it was low under irrigation production season. Although this may reflect a different relationship between DTM and NUE in the two seasons, we cannot rule out that the low correlation between the two traits under irrigation may be caused by the difference in harvesting times between seasons. Maturing of the late cultivars in the irrigation production season overlapped with the onset of the rainfed production season, and because of the high rainfall, we had to harvest the experiments before all cultivars (especially the late cultivars) matured. This relative early harvesting of the late cultivars may have reduced the contribution of maturity to yield and NUE and thus may have affected the correlation between the two traits.

The QTLs detected for Vmax, AUC, DTM and NUE shared the same peak marker on chromosome V in our CxE linkage mapping study (Chapter 3). Similarly, Ospina et al. (2016) detected QTLs for canopy development traits in the SH x RH diploid biparental potato population that shared the same peak marker with the above-mentioned region of chromosome V. This co-location and sharing of the same peak marker underscores the importance of this region in influencing NUE and NUE-related traits. Although this region can be used as a potential target for NUE improvement, the influence of maturity is substantial. Thus, priority should be given to see whether the linkage between the maturity-driving CDF1 gene and NUE and related traits can be separated.

AUC on chromosome III and TNPP and AP2 on chromosome VI co-located with QTLs for NUE. These two genomic regions may be useful for NUE improvement in potato other than chromosome V, and these are independent of maturity type. Trait-specific stable QTLs (QTLs 
for one specific trait observed in more than one environment) were detected for various NUE related traits in different environments. Stable QTLs for TYPP with peak markers solcap_snp_c2_26796 on chromosome IV and marker PotVar0060022 on chromosome XI were observed at two low $\mathrm{N}$ level environments under irrigation production system, indicating the QTLs may be low $\mathrm{N}$ as well as irrigation season dependent. This suggests the presence of QTL x Environment interaction. However, the difference in overall number of identified QTLs between low and high $\mathrm{N}$ level is lower than the QTL number difference between the two production seasons, suggesting QTL x N level interaction was lower than the QTL x production season interaction, in line with the stronger contribution of production system to the total genotype $\mathrm{x}$ environment interaction compared to $\mathrm{N}$ levels, as already discussed. Similarly, studies in rapeseed using multi-environment trials that included various locations and growing seasons showed that a large number of QTLs were stable across $\mathrm{N}$ levels, while Genotype $\mathrm{x}$ Trial interaction was strong and most of the QTLs were specific to a single trial (Bouchet et al., 2016). In a bread wheat QTL x environment interaction study, Kuchel et al. (2007) reported that a large portion of the $\mathrm{G} \times \mathrm{E}$ interaction could be explained by interaction of the QTLs with climatic factors.

To identify QTLs, various gene models were used in different association studies. Most QTL studies focus on estimating the additive effect of the QTL, assuming absence of interaction among QTLs (Bocianowski and Krajewski, 2009; Rovaris et al., 2011). However, epistatic and dominance effects also play a very important role in controlling the expression of quantitative traits (Bocianowski, 2013). In this study, we use additive, simplex and duplex dominant, as well as general models; most of the MTAs (including MTAs identified for NUE, AUC, TNPP, and TYPP) were detected using dominant genetic models. This indicates that the source of heritable variation for the identified MTAs is mostly due to dominant gene action or due to the interaction of alleles at a single locus, and that dominant gene effects are important in controlling potato NUE and NUE related traits. Gopal (1998) in his study on early generation of potato general combining ability also reported that non-additive gene effects were more important than additive gene effects in determining potato tuber yield and yield components. Previous selection which may have narrowed the genetic base of the studied genotypes may be one of the possible causes for greater non-additive genetic variance effect for various traits (Plaisted et al., 1962). Killick and Malcolmson (1973) in their potato combining ability study reported that Specific Combining Ability (SCA) is more important than General Combining Ability (GCA) in most traits, suggesting that traits subjected to directional selection would be 
expected to show little additive variance. Our results endorse this concept as most of the cultivars used in our panel have been subjected to selection for tuber yield and other tuber quality traits under various potato breeding programs.

Combining QTLs that control traits of interest from different genomic regions in a single genetic background is a challenging mission in plant breeding. The use of markers for loci that accumulate several traits may increase the QTL pyramiding efficiency in marker assisted selection. In this study, several traits that contribute to NUE mapped to the same regions on the genome and can thus be introduced in a new cultivar together, thus reducing the challenge of collecting QTLs that control traits of interest from different genomic regions.

In conclusion, genome-wide association mapping detected both stable and environment specific QTLs for NUE and NUE related traits. NUE-related traits such as DTM, Vmax and AUC had a strong positive correlation with tuber yield and yield component traits. The colocalization of QTLs for these traits with NUE QTLs suggests that these can be used for indirect assessment of some tuber yield and yield component traits. Multi-trait chromosome regions have been identified on chromosome III, V and VI associated with NUE and NUE related traits. Markers found in the aforementioned chromosome regions could be used for future improvement of NUE and related traits through marker assisted selection. However, to use the markers detected on chromosome $\mathrm{V}$ efficiently, it would help to be able to separate the trait earliness from the other traits. Our result demonstrated that the effect of production season was greater than the effect of N levels on NUE and NUE related traits under our experimental conditions. Still, critical genomic regions associated with NUE that were stable across potato populations were identified. 


\section{Chpater 5}

\section{Genotype-by-Environment interaction for nitrogen use efficiency of potato (Solanum tuberosum L.) under different growing conditions in North western Ethiopia.}

Baye Berihun Getahun ${ }^{1,2}$, Mulugeta Atinaf Tiruneh ${ }^{4}$, Marcos Malossetti ${ }^{3}$, Richard GF Visser ${ }^{1,2}$, C. Gerard van der Linden ${ }^{1,2}$

${ }^{1}$ Graduate School Experimental Plant Sciences, Wageningen University \& Research, PO Box 386, 6700 AJ Wageningen, The Netherlands;

${ }^{2}$ Plant Breeding, Wageningen University \& Research, PO Box 386, 6700 AJ Wageningen, The Netherlands;

${ }^{3}$ Biometris, Wageningen University and Research, PO Box 16, 6700 AA Wageningen, The Netherlands;

${ }^{4}$ Ethiopian Institute of Agricultural Research, PO Box 25, Addis Ababa, Ethiopia 


\begin{abstract}
Understanding genotype-by-environment interaction (GE interaction) and the way to exploit it, is important in developing crop breeding strategies for potato. The objectives of this study were to determine the GE interaction and stability of nitrogen use efficiency (NUE) in potato cultivars, identify promising genotypes, and evaluate test environments. The study was conducted in eight environments representing low and high nitrogen levels combined with rainfed and irrigation production conditions at three different locations in Ethiopia; DebreTabor, Injibara and Koga. These are located in potato growing areas of North western Ethiopia. Eighty-one European commercial potato cultivars were evaluated using a split plot design with two replications. Data were analysed using the genotype, and the genotype and environment (GGE) biplot model. The GGE analysis identified two mega-environments that coincide with the two production systems. Three promising cultivars (Kuras, Asterix and Desirée) in the rainfed mega-environment, and two cultivars in the irrigation mega-environment (Hermes and Kuroda) combined good mean performance with stability in NUE. Testing environments for proper selection of genotypes based on representativeness and discriminating ability were also identified. The high $\mathrm{N}$ level environments at both Debre-Tabor and Injibara were the most suitable environments in discriminating the potato cultivars and being representative test environments for NUE evaluation in the rainfed mega-environment. The low $\mathrm{N}$ environment at Koga was the most suitable environment in discriminating and representing the irrigation mega-environment.
\end{abstract}

Key words : Genotype-by-environment interaction, mega-environment, growing conditions, NUE, potato 


\section{Introduction}

Crop genotypes grown in different environments encounter considerable fluctuations in yield, especially when the growing environments are markedly different. This instability of crop performance with a changing growing environment is referred to as genotype-by-environment interaction (GE interaction). Bowman (1972) defined GE interaction as a change in the relative performance of a "trait" of two or more genotypes in two or more environments. The occurrence of GE interaction in a crop improvement program potentially influences the selection and varietal development for a target set of environments, especially when the rank order changes among the genotypes (Navabi et al., 2006). Understanding the environmental and genotypic causes of GE interaction is important to many plant breeding goals, including ideotype strategies and parent selection based on traits of interest (Jackson et al., 1998; Yan and Hunt, 1998). Various statistical models have been proposed and used to analyze and further partition the GE interaction. The additive main effect and multiplicative interaction (AMMI) (Zobel et al., 1988) approach, and genotype plus genotype-by-environment interaction (GGE) approach as proposed by Yan et al., (2000) have been the most widely used models to analyze multi-environment data. However, GGE best fits a mega environment analysis, genotype and evaluation of test environments (Amira et al., 2013; Yan et al., 2007).

In Ethiopia, potato is cultivated both in the rainy season under rainfed conditions and in the dry season using irrigation. The two production systems have different production constraints; higher maximum and lower minimum average temperature are the predominant irrigated potato production system constraints, while incidence of late blight is the prevailing production constraint in the rainfed potato production system (Yigzaw et al., 2008). Despite the difference in production constraints and climatic conditions between the two production systems, farmers grow the same cultivars for both rainfed and irrigation production systems. The Ethiopian potato breeding program has developed a number of improved potato varieties. However, these improved varieties were released only for rainfed production systems under high input conditions. Nevertheless, according to Mulat (1999) the amount of fertilizer applied by most Ethiopian farmers is below the recommended level. For instance, from the total cereal production areas only $35 \%$ receive chemical fertilizer. The average fertilizer use of Ethiopia as a country for all crops is about $17 \mathrm{~kg} / \mathrm{ha}$, which is very low by any standard (Agriculture For Impact, 2014). For these type of environments, potato varieties that have higher nitrogen use efficiency (NUE) under low $\mathrm{N}$ input conditions are required. NUE has critical economic and environmental values. Although it is difficult to assess the economic costs associated with 
inefficient $\mathrm{N}$ use, Raun and Johnson (1999) have estimated that every $1 \%$ increase in $\mathrm{N}$ fertilizer uptake efficiency of cereal crops would save approximately US $\$ 2.3$ billion in a year on fertilizer costs in the world. Despite its importance, the main mechanisms of NUE and the genetic factors involved are poorly understood (Basra and Goyal, 2002). Partly this may be due to the inherent complex nature of NUE, as it is a function of several interacting genetic and environmental factors. The existence of genetic variation among genotypes for NUE at least suggests that improving NUE through breeding is an option. Information about the relative importance of various traits related to NUE is vital for efficient breeding for NUE, but genetic studies of NUE are hampered by interaction with environmental factors. very often, the environmental variation of NUE supersedes the genotypic contribution to NUE performance in field-grown crops in particular (Bertin and Gallais 2000; Dawson et al., 2008). Factors like temperature, water availability, and soil type may affect NUE by affecting crop growth as well as the availability of $\mathrm{N}$ in the soil by affecting mineralization of soil organic matter, organic fertilizer and leaching of soil nitrate (Agostini et al, 2010). Increased insight in GE interaction of NUE related genetic factors in potato can therefore be very helpful.

Often in GE interaction studies, crop yield is used as the target trait to evaluate the suitability of the test environments and the superiority of the cultivars in each environment, because yield has a critical economic value and is used in assessing the superiority of the genotypes. In this study, we selected nitrogen use efficiency (NUE) as a target trait. According to Barraclough et al. (2010), yield is a measure of nitrogen use efficiency, and the definition of NUE used in this study is the ratio of dry potato tuber yield and the available nitrogen ( $\mathrm{N}$ in the soil + applied $\mathrm{N})$. GE interaction considering $\mathrm{N}$ level as part of environment and NUE as a target trait is vital to exploit potato genetic resources efficiently and identify optimal test environments and superior genotypes for NUE improvement in different fertility level and production systems. So far, no work has been done on the suitability of test environments and stability of potato cultivars for NUE in different production conditions, including low $\mathrm{N}$ level and high $\mathrm{N}$ level as part of the environment. The aim of this study was (a) to evaluate the influence of locations, $\mathrm{N}$ levels, and production conditions on NUE and on stability of potato cultivars and (b) to identify optimal testing environments. 


\section{Materials and methods}

\section{Plant Materials}

A total of 81 Dutch potato cultivars were used in this experiment. The cultivars were released from different potato breeding companies for different purposes and in different years (Berloo et al., 2007) and are common cultivars in the North-western European potato market (Supplementary Table 1).

\section{Description of experimental area}

The experiments were conducted at three different locations in North-western Ethiopia: at Injibara and Debre-Tabor in 2013 under rainfed conditions, and at Koga and Injibara in 2015 in the dry season under irrigation. The locations are situated in the Amhara region (Figure 1), which is the major potato growing area of the country accounting for about $40 \%$ of the Ethiopian potato farmers (CSA 2008/2009). Debre-Tabor and Injibara are located at higher altitudes. Debre-Tabor is located at 2650masl with expected average annual rain fall of $1500 \mathrm{~mm}$, and Injibara is located 2600masl with $2300 \mathrm{~mm}$ annual average rain fall. However, Debre-Tabor is relatively dry highland, while Injibara is a wet highland area. Although the intensity was high, the rainfall distribution was good for potato production in both locations, during the main rainfed production season.

The irrigation experiments in 2015 were conducted during the dry season (February to June). The irrigation period of the year in Ethiopia is characterized by dry weather, high day temperature, low night temperature and low disease incidence. The two experimental sites are located in different agro-ecologies. Koga is located at mid altitude (1900 masl), while Injibara is located at higher altitude (2600masl). Irrigation water was applied every week in both locations. There was no scarcity of irrigation water especially at Koga, but at Injibara there was scarcity of irrigation water particularly at the beginning of the experiment. The environmental variation experienced from location to location even within a short distance, and from rainfed production season to irrigation production season are among the most dominant features of the Ethiopian environmental conditions (EMA, 1988). We defined eight target environments for the data analysis. Each location combined with a production season and $\mathrm{N}$ level was considered as a separate target environment making a total of eight test environments for this study. Description of the test locations and the eight defined environments are presented in Table 1 and Table 2, respectively. 


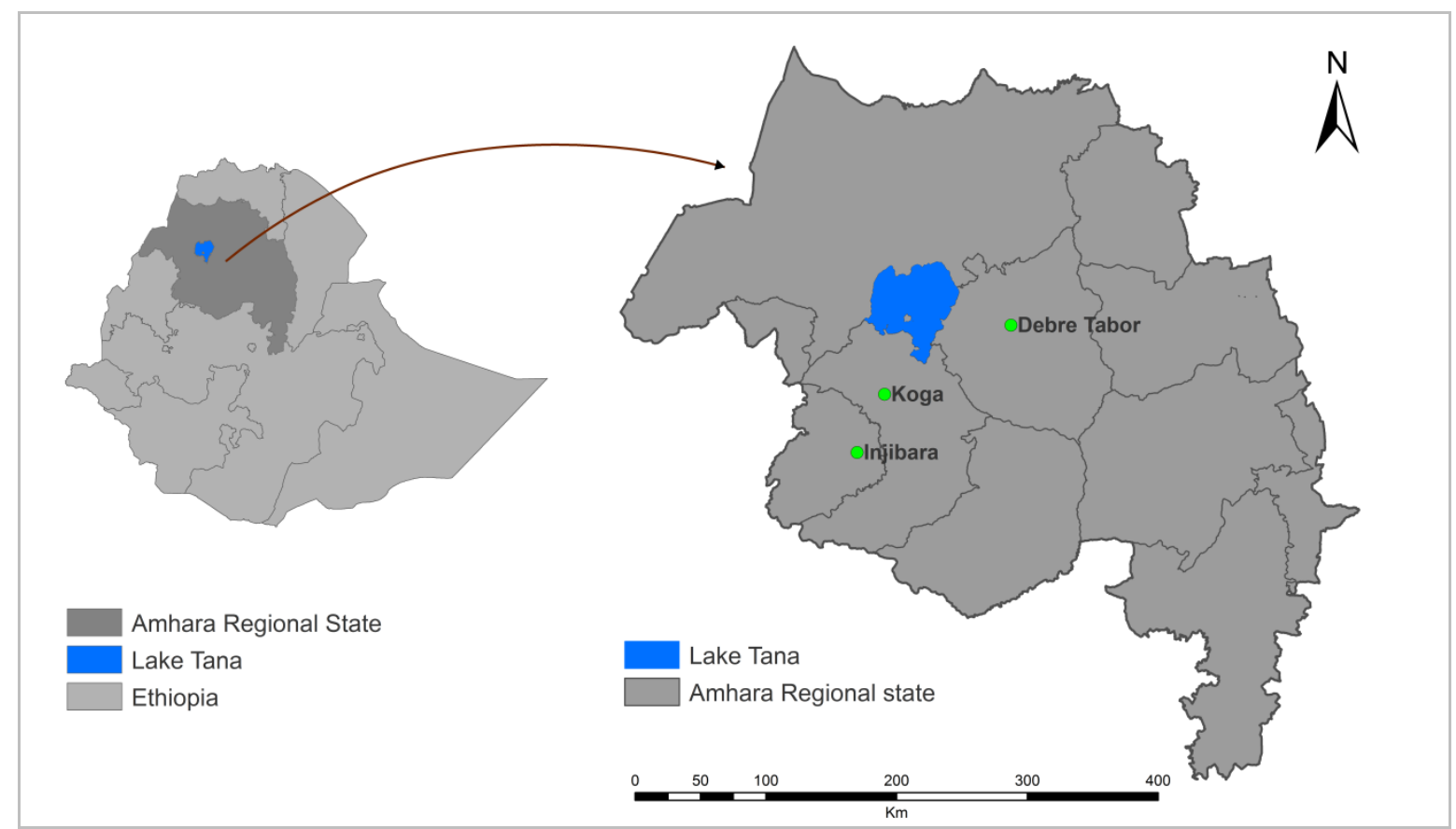

Figure 2. Location map of the study area

Table 1. Description of the experimental locations used in this study.

\begin{tabular}{lccccccccc}
\hline & \multicolumn{2}{c}{ Geographical position } & & & \multicolumn{2}{c}{ Temperature $\left({ }^{\circ} \mathrm{C}\right)$} & $\begin{array}{c}\text { Soil } \\
\text { type }\end{array}$ & Soil pH \\
Locations & Latitude & Longitude & $\begin{array}{c}\text { Altitude } \\
\text { (masl) }\end{array}$ & $\begin{array}{c}\text { Ave. } \\
\text { rain } \\
\text { fall }(\mathrm{mm})\end{array}$ & Min & Max & & & \\
\hline D/Tabor & $11.89^{\circ} \mathrm{N}$ & $38.04^{\circ} \mathrm{E}$ & 2650 & 1500 & 11.8 & 23 & luvisol & 5.2 \\
Injibara & $10.85^{\circ} \mathrm{N}$ & $36.8^{\circ} \mathrm{E}$ & 2600 & 2300 & 8.0 & 22 & Acrisol & 4.8 \\
Koga & $11.37^{\circ} \mathrm{N}$ & $37.12^{\circ} \mathrm{E}$ & 1900 & 1400 & 9.0 & 32 & Nitosol & 5.4 \\
\hline
\end{tabular}

Table 2. Adopted environments with the involvement of different factors

\begin{tabular}{lllll}
\hline Environments & Location & N-levels & Year & Production condition/season \\
(E) & & & \\
\hline E1 & Debre-Tabor & Low N & 2013 & Rainfed \\
E2 & Debre-Tabor & High N & 2013 & Rainfed \\
E3 & Injibara & Low N & 2013 & Rainfed \\
E4 & Injibara & High N & 2013 & Rainfed \\
E5 & Injibara & Low N & 2015 & Irrigation \\
E6 & Injibara & High N & 2015 & Irrigation \\
E7 & Koga & Low N & 2015 & Irrigation \\
E8 & Koga & High N & 2015 & Irrigation \\
\hline
\end{tabular}

E1= Debre-Tabor low N(Rainfed), E2= Debre-Tabor high N(Rainfed), E3= Injbara low N (Rainfed), E4=Injbara high N (Rainfed), E5=Injbara low N (irrigation), E6= Injbara high N (irrigation), E7= Koga low N (irrigation), E8=Koga high N (irrigation), $\mathrm{LN}=$ low $\mathrm{N}\left(40 \mathrm{~kg} \mathrm{ha}^{-1}\right), \mathrm{HN}=\operatorname{high~} \mathrm{N}\left(120 \mathrm{~kg} \mathrm{ha}^{-1}\right)$. 


\section{Field trials and experimental design}

The field experiments were conducted with a similar experimental setup in all locations under rainfed and irrigation production conditions. The trials had a split-plot design with two replications, where the main plots were assigned to the low and high $\mathrm{N}$ rates $(40 \mathrm{~kg} / \mathrm{ha}$ and $120 \mathrm{~kg} / \mathrm{ha}$ ) and the sub-plots to the genotypes. Each experimental plot consisted of 10 tubers planted in one row with an intra-row of $0.30 \mathrm{~m}$ and $0.75 \mathrm{~m}$ spacing between rows, and each experimental plot was bordered by a reference potato cultivar. Application of irrigation water, pest and disease management, weeding and ridging and other cultivations was done following the recommendation of each location and when required. The total amounts of N (40 and 120 $\mathrm{kg} / \mathrm{ha}$ ) included the available $\mathrm{N}$ in the soil and application of commercial $\mathrm{N}$ fertilizer in the form of urea and di-ammonium phosphate (DAP). Phosphorus fertilizer was applied following the recommendation for each location in the form of DAP and tri-supper phosphate (TSP). The whole $\mathrm{P}$ source was applied at planting while $\mathrm{N}$ application was split in two: a week after emergence and at the start of flowering.

\section{Trait measurements}

Phenotypic data were collected for several agronomic and physiological traits. Harvesting was conducted when the last cultivar reached physiological maturity $(90 \%$ of the haulm tissue brown), and eight plants per plot were harvested and used to evaluate the tuber yield plant ${ }^{-1}$, yield- related traits and NUE. To calculate NUE, we measured first the specific gravity of the tuber. Specific gravity (SG) was determined using the tuber specific gravity procedure of weight in air and under water (Murphy and Goven, 1959). In evaluating the SG of each variety, healthy and marketable-sized grade (20mm and above) tubers were selected randomly from each variety harvest. Then, tubers were cleaned, and weighed both in air and water following the procedure of (Murphy and Goven, 1959).

$$
S G=\frac{W_{1}}{W_{1}-W_{2}}
$$

where $\mathrm{SG}=$ specific gravity of the material, $\mathrm{W}_{1}=$ weight in air of the sample tuber, in $\mathrm{g}$ and $\mathrm{W}_{2}=$ Weight of the sample completely immersed in water, in grams. we determined tuber dry matter in percent (TDM\%) indirectly from SG using empirical conversion factors following the equation of Kleinkopf et al. (1987): solid (Dry matter \%) $=-214.9206+(218.1852 \times$ SG). And 
then Tuber dry weight (TDW) was estimated indirectly from specific gravity and tuber dry matter content, using the following formula: $T D W=\frac{T D M \% * T F W}{100}$

Where TDW $=$ Tuber dry wieght in $\mathrm{g}$, TDM $=$ Tuber dry matter percentage, $\mathrm{TFW}=$ Tuber fresh weight in g. Finally, NUE was determined as the tuber dry matter production, or dry weight of the tubers $\mathrm{ha}^{-1}$, per unit of $\mathrm{N}$ supplied $\mathrm{ha}^{-1}(\mathrm{~N}$ in the soil + applied $\mathrm{N})$.

\section{Data analysis}

The analysis of variance of NUE data for each trial was done using a split-plot design with GenStat $18^{\text {th }}$ edition software. The adjusted NUE means of each trial was used in a combined analysis of variance to evaluate the main effect of environment $(E)$, genotype $(G)$, and genotype-by-environment interaction (GE) variances. Further partitioning and analysis of GE interaction was carried out with GGE biplot model using GGE software (Yan, 2001). The GGE biplot was constructed using the first two principal components (PC1 and PC2) derived from environment-centred NUE data ((Yan et al., 2000). Environment-centred data is the data with the grand mean and the environmental effects removed from the data as defined by the following GGE model:

$Y i j-\mu-\beta j=\lambda_{1} \xi_{i 1} \eta_{i 1}+\lambda_{2} \xi_{i 2} \eta_{j 2}+\varepsilon_{i j}$ Where $Y i j$ is the measured mean of genotype $i(=1,2, \ldots, n)$ in environment $j(=1,2 \ldots, m), \mu$ is the grand mean, $\beta j$ is the main effect of environment $j, \lambda_{1}$ and $\lambda_{2}$ are the singular values (SV) for the first and second principal component (PC1 and PC2), respectively, $\xi_{i 1}$ and $\xi_{i 2}$ are eigenvectors of genotype i for PC1 and PC2, respectively, $\eta_{1 j}$ and $\eta_{2 j}$ are eigenvectors of environment $j$ for PC1 and PC2, respectively, $\varepsilon_{i j}$ is the residual associated with genotype $i$ in environment $j$.

\section{Results}

\section{Variance analysis}

The individual and combined analysis of variance for tuber yield revealed highly significant differences $(\mathrm{P} \leq 0.001)$ among genotypes in all environments except in $\mathrm{E} 8$ (Koga, high $\mathrm{N}$ in 2015) (Table 3). The experimental coefficients of variation (CV) were relatively low, ranging from 0.15 to 0.25 , except in E8 (CV 0.36). The irrigation production season test environments (E5, E6, E7 and E8) showed higher tuber yield and NUE performance compared to rainfed production season test environments (E1, E2, E3 and E4) (Table 3). However, within the irrigation production season, test environments at Injibara (E5 and E6) have shown lower 
performance compared with test environments at Koga (E7 and E8). The low tuber yield and NUE performance of our cultivars at Injibara in the irrigation production season may be attributed to shortage of irrigation water especially at early stage (emergence and growth) stage of the crop. Genotype G61 Navigator had the highest tuber yield (0.6 kg/plant). All other genotypes yielded $0.35 \mathrm{~kg}$ or more except G6 Annabelle (0.32 kg/plant). Among the environments E8 had the highest mean yield (0.89 kg/plant) (Supplementary Table 2).

Similarly, the individual analysis of variance for NUE was significantly different $(\mathrm{P} \leq 0.05)$ among genotypes in E8 and highly significantly different $(\mathrm{P} \leq 0.001)$ in all other environments (Table 3). The coefficients of variations (CV) were a bit higher in irrigation experiments compared to rainfed; this higher $\mathrm{CV}$ may be related with irrigation water management. As the experiments were conducted using furrow irrigation at field conditions there may be differences in seepage and other factors between plots that may have caused higher CV values.

Test environments in the same production season were positively correlated with each other, while the correlation between test environments of most rainfed and irrigation season test environments were mostly low (Table 4), indicating GE interaction.

The combined analysis of variance over environments revealed that potato NUE is significantly $(\mathrm{P}<0.001)$ affected by the environment $(\mathrm{E})$, genotype $(\mathrm{G})$ and genotype-byenvironment interaction (GE) (Table 5). The environment accounted for $79.6 \%$ of the total sum of squares (SS) of $(\mathrm{G}+\mathrm{E}+\mathrm{GE})$ variation, which is the largest contribution to the total variation. The genotype and genotype-by-environment interaction respectively accounted for only $4.1 \%$ and $16.3 \%$ of the total sum of square variation (Table 5). The significant effect of the GE interaction in the combined analysis of variance suggests that the genotypes had variable performance in the tested environments (different best performers at different environments). 
Table 3. Mean squares of individual analysis of variance by environment for tuber yield and NUE of 81 potato genotypes in 8 environments

\begin{tabular}{|c|c|c|c|c|c|c|c|c|c|}
\hline \multirow[b]{2}{*}{ Source of variation } & \multicolumn{9}{|c|}{ Mean squares of individual environment analysis of variance } \\
\hline & Df & E1 & E2 & E3 & E4 & E5 & E6 & E7 & E8 \\
\hline Replication (a) & 1 & 0.4 & 0.11 & 0.014 & 0.28 & 0.075 & 0.23 & 0.45 & 0.13 \\
\hline Replication (b) & 1 & 453.9 & 12.69 & 554.2 & 301.61 & 1323.6 & 479.27 & 18974 & 5454.4 \\
\hline Genotype (a) & 80 & $0.01 * * *$ & $0.02 * * *$ & $0.003 * * *$ & $0.014 * * *$ & $0.014 * * *$ & $0.021 * * *$ & $0.045 * * *$ & 0.12 \\
\hline Genotype (b) & 80 & $243.43 * * *$ & $38.98 * *$ & $430.9 * * *$ & $64.10 * * *$ & $438.2 * * *$ & $83.68 * * *$ & $1454.3 * * *$ & $416.8^{*}$ \\
\hline Residual (a) & 80 & 0.003 & 0.005 & 0.001 & 0.004 & 0.004 & 0.005 & 0.025 & 0.09 \\
\hline Residual (b) & 80 & 89.39 & 20.04 & 102.6 & 21.64 & 194.4 & 34.94 & 471.5 & 288.2 \\
\hline mean (a) $\left(\right.$ kg plant $\left.^{-1}\right)$ & & 0.31 & 0.44 & 0.22 & 0.43 & 0.35 & 0.42 & 0.63 & 0.89 \\
\hline mean(b) $\left(\mathrm{kg} \mathrm{ha}^{-1} \mathrm{~kg}^{-1} \mathrm{ha}^{-1}\right)$ & & 37.06 & 14.60 & 41.21 & 15.64 & 41.21 & 15.63 & 101.31 & 51.11 \\
\hline $\mathrm{CV}(\%)(\mathrm{a})$ & & 17.6 & 16.4 & 16.5 & 14.5 & 17.7 & 17.1 & 25.4 & 35.6 \\
\hline $\mathrm{CV}(\%)(\mathrm{b})$ & & 25.5 & 30.7 & 24.6 & 29.7 & 35.4 & 37.3 & 21.4 & 33.2 \\
\hline
\end{tabular}

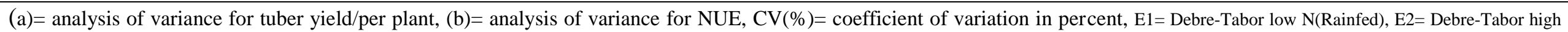

$\mathrm{N}$ (Rainfed), E3= Injbara low N (Rainfed), E4=Injbara high N (Rainfed), E5=Injbara low N (irrigation), E6= Injbara high N (irrigation), E7= Koga low N (irrigation), E8=Koga high N (irrigation) 
Table 4. Pearson correlation coefficients among the 8 potato testing environments based on NUE data

\begin{tabular}{|c|c|c|c|c|c|c|c|c|}
\hline & E1 & E2 & E3 & $\mathrm{E} 4$ & E5 & E6 & E7 & E8 \\
\hline E1 & - & & & & & & & \\
\hline E2 & $0.54^{* * *}$ & - & & & & & & \\
\hline E3 & $0.37^{* * *}$ & $0.49^{* *}$ & - & & & & & \\
\hline E4 & $0.41^{* * *}$ & $0.50^{* *}$ & $0.43^{* * * *}$ & - & & & & \\
\hline E5 & $0.08^{\mathrm{ns}}$ & $0.01^{\mathrm{ns}}$ & $-0.15^{\mathrm{ns}}$ & $0.09^{\mathrm{ns}}$ & - & & & \\
\hline E6 & $0.03^{\mathrm{ns}}$ & $0.05^{\mathrm{ns}}$ & $-0.18^{\mathrm{ns}}$ & $-0.03^{\mathrm{ns}}$ & $0.72^{* * *}$ & - & & \\
\hline E7 & $-0.0003^{\text {ns }}$ & $0.01^{\mathrm{ns}}$ & $-0.2^{\mathrm{ns}}$ & $-0.16^{\mathrm{ns}}$ & $0.29^{* *}$ & $0.37^{* *}$ & - & \\
\hline E8 & $-0.05^{\mathrm{ns}}$ & $-0.042^{\text {ns }}$ & $-0.29^{* *}$ & $-0.12^{\text {ns }}$ & $0.14^{\mathrm{ns}}$ & $0.17^{\mathrm{ns}}$ & & - \\
\hline
\end{tabular}

E1= Debre-Tabor low N(Rainfed), E2= Debre-Tabor high N(Rainfed), E3= Injibara low N (Rainfed), E4=Injibara high N (Rainfed), E5=Injibara low N (irrigation), E6= Injibara high N (irrigation), E7= Koga low N (irrigation), $\mathrm{E} 8=\mathrm{Koga}$ high $\mathrm{N}$ (irrigation). ${ }^{* * *}=$ significant at $\mathrm{P} \leq 0.001, * *=$ significant at $\mathrm{P} \leq 0.01$, ns $=$ not significant

Table 5. Combined analysis of variance for NUE $(\mathrm{kg} / \mathrm{kg})$ of 81 potato cultivars for all 8 environments taken together

\begin{tabular}{lllllll}
\hline $\begin{array}{l}\text { Source of } \\
\text { Variation }\end{array}$ & DF & SS & Ms & V & P & variation (\%) \\
\hline Environment (E) & 7 & 901752.8 & 128821.8 & 718.35 & $\leq 0.001$ & 79.6 \\
Genotype (G) & 80 & 46419.3 & 580.2 & 3.24 & $\leq 0.001$ & 4.1 \\
GE & 560 & 184643.6 & 329.7 & 1.84 & $\leq 0.001$ & 16.3 \\
Residual & 647 & 116026.9 & 179.3 & & & \\
Total & 1295 & 1250565 & & & & \\
\hline Mean & & & 40.1 & & & \\
CV $(\%)$ & & 33.4 & & & \\
\hline
\end{tabular}

$\mathrm{DF}=$ Degrees of freedom, $\mathrm{SS}=$ sum of square, $\mathrm{MS}=$ mean of square, $\mathrm{V}=$ variance, $\mathrm{GE}=$ genotype-by- environment interaction, $\mathrm{CV} \%=$ coefficient of variation, $\mathrm{P}=$ significance level

\section{Mega environment analysis}

Considering the mega-environment components of a target region for a specific crop is a precondition for determining proper approaches of genotype evaluation and cultivar recommendation. The vector view of the GGE biplot for NUE of 81 potato cultivars evaluated in 8 environments is shown in Figure 2a. The environments are connected to the biplot origin by the vectors. The percentages of GGE explained by PC1 and PC2 were $31.4 \%$ and $25.8 \%$ respectively, and the biplot explained $57.2 \%$ of the total variation due to $\mathrm{G}$ and GE using an environment-standardized model. In this case the total variation is referred to as the variation 
due to the $\mathrm{G}$ and $\mathrm{GE}$, because the variation due to the environment main effect is excluded by this model. The GGE and especially PC1 clearly separated the two production seasons (irrigation and rainfed). All environments within the same production season appeared to be correlated (as indicated by the less than $90^{\circ}$ angle between them), while the two production seasons appeared to be negatively correlated (as indicated by more than $90^{\circ}$ angles (Yan, 2002), in agreement with the correlations presented in Table 4. The negative correlations between groups of test environments are a strong indication that the environments can be grouped in two different mega-environments.

Mega-environments can be defined by which-won-where patterns, and the GGE biplot is an effective graph to show these in a genotype-by-environment dataset. The biplot in Figure $2 b$ is similar to the biplot Figure $2 \mathrm{a}$ except that the environment vectors were removed and a polygon with lines perpendicular to the polygon sides was added. The polygon was drawn based on cultivars placed away from the biplot origin so that all cultivars are included in the polygon. The 8 environments fall into two sectors delineated by the straight lines radiating from the biplot origin and perpendicular to sides of the polygon.

The sector delineated by lines 1 and 7 comprises the four rainfed environments E1, E2, E3 and E4. The genotype G43 (Kuras) is placed on the vertex of the polygon for this sector, and is therefore considered the most nitrogen efficient cultivar for this group of environments (see also supplementary Table 1). The second sector is defined by the radiate lines 6 and 7, and includes the four environments of the irrigation production season (E5, E6, E7, and E8). Genotype G3 is on the vertex for this section suggesting that G3 (Agria) was the winner at these environments, and this genotype indeed has the highest NUE in the irrigation production season trials. Thus, we identified two mega-environments (the irrigation and rainfed), and the test-environment and genotype evaluation was done separately for each of the two mega environments. 

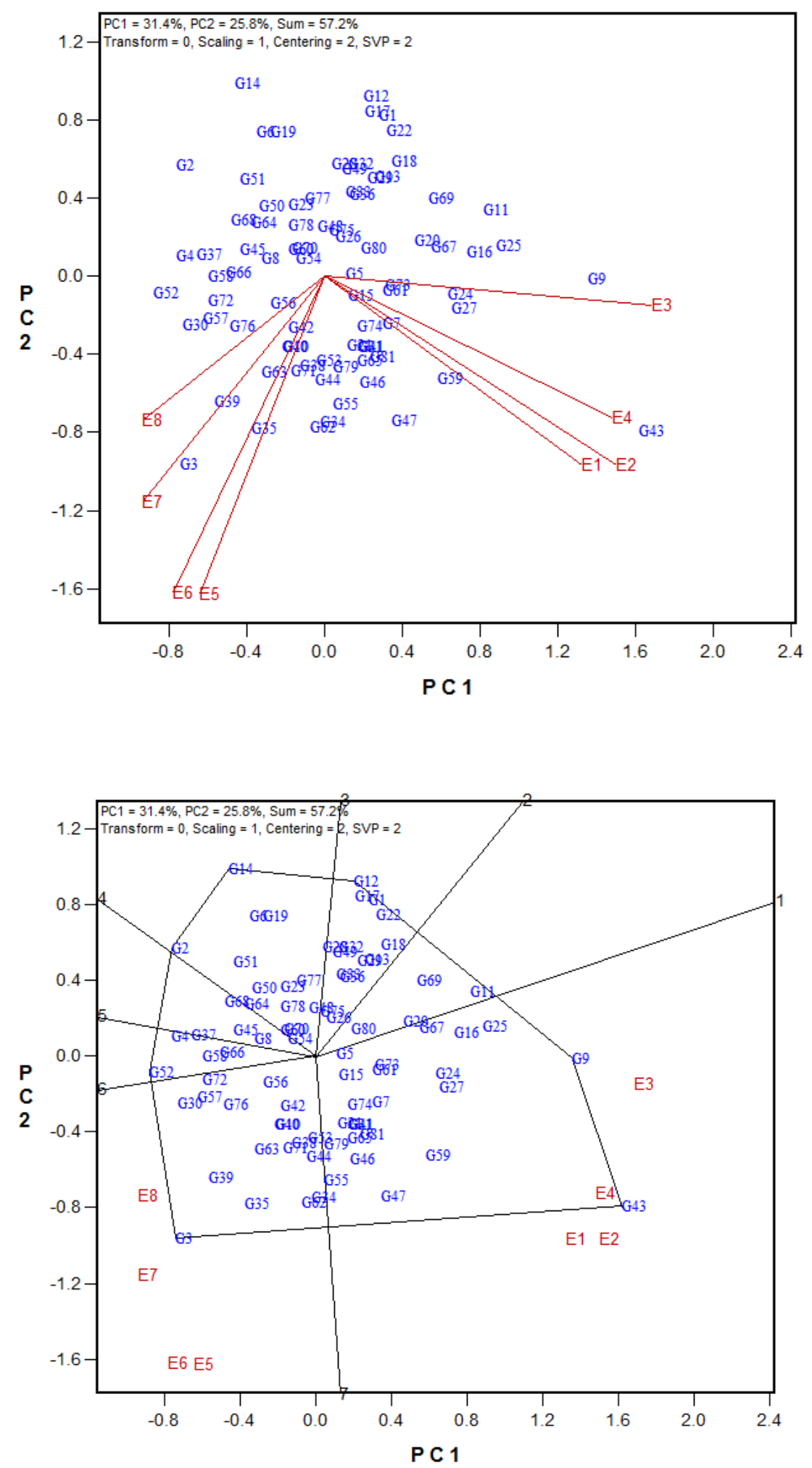

Figure 2: GGE biplot for NUE. Figure 2a is the vector-view GGE biplot based on environment-focused singular value partitioning, showing the interrelationship among test environments. Figure $2 b$ is the "which-won-where" view of the GGE biplot under each mega-environment constructed based on environment-centered and symmetrical singular value partitioning. See codes of environments and genotypes in Table 1 and Chapter 5 supplementary Table 2, respectively. 


\section{Test environment evaluation}

Evaluating the test environments is helpful to identify test environments that can effectively identify superior genotypes and representative test environments for a mega environment, in our case for the rainfed or the irrigated production season. The GGE biplots in Figure 3 and 4 were constructed based on environment-focused singular value partitioning for the two production seasons separately, in order to visualize the representativeness and genotypediscriminating power of the test environments in these mega environments. In the two biplots the single arrowed red line that passes through the biplot origin is the average environment axis (AEA), and the small red circle on the AEA represents the average environment. The double arrowed blue line perpendicular to the AEA represents the average environment coordination (AEC). The representativeness of the environments is measured by the cosine of the angle between the test environments and AEA; the closer the test environment is to the average environment the more desirable it is as core testing environment (Yan et al., 2007). The vector length from the origin of the biplot to the environments is a measure of the discriminating power of the environments. Figure 3 demonstrates that all test environments are positively correlated with the AEA, and therefore they were all representative but to different degrees (see also Table 6). E2 and E4 (the high N environments) were closest to the average environment with good discriminating ability (long vector lengths) suggesting that these are the test environments that best represent the rainfed mega environment, while E1 and E3 (the low $\mathrm{N}$ environments) were well-discriminating but less representative environments.

Similar to the rainfed mega environment, all four test environments in the irrigation megaenvironment were correlated with the AEA of the mega environment (Figure 4 and Table 7). E7 was more representative, while E8 was less representative. The two testing environments at Injibara (E5 and E6, low and high N) were strongly correlated with each other, indicating their similarity in discriminating the genotypes and representativeness of the megaenvironment. 


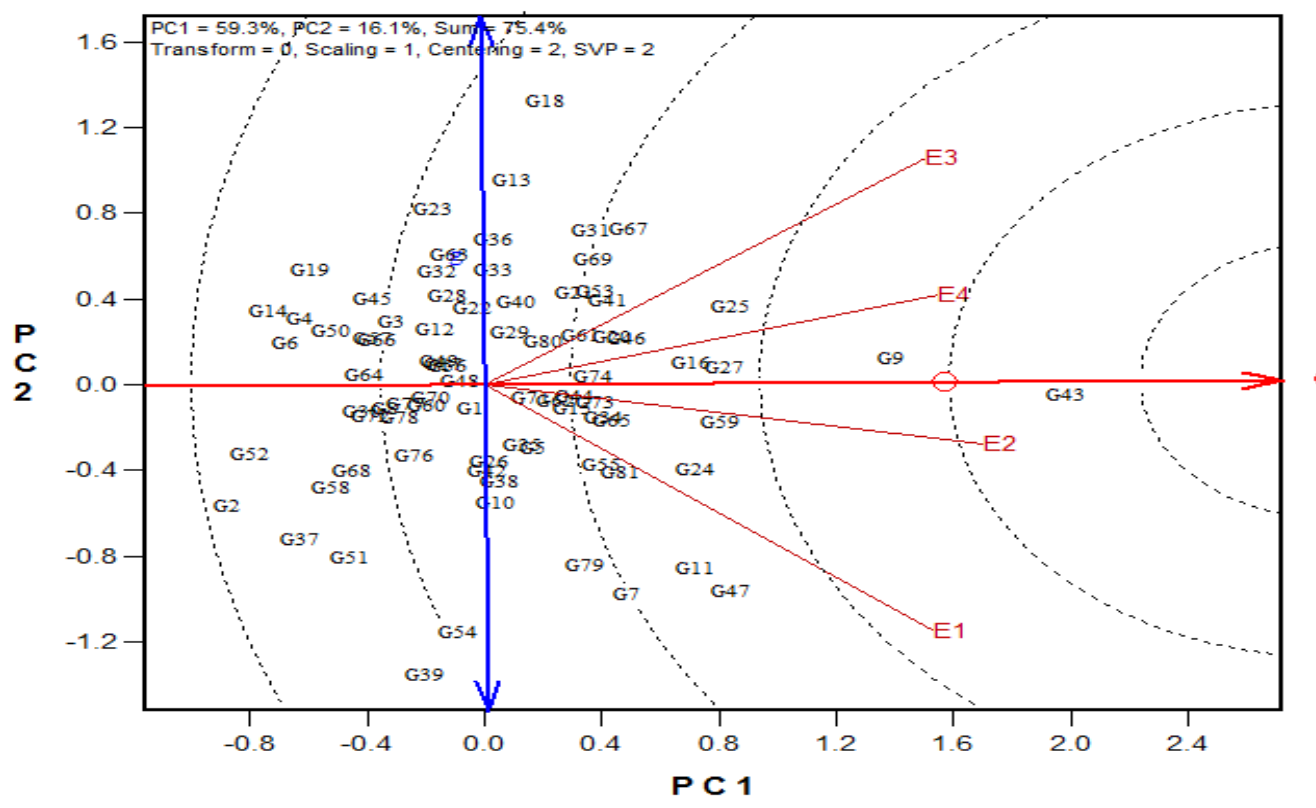

Figure 3. The Representativeness vs Discrimination view of the GGE biplot for test environments based on NUE value of potato cultivars in rainfed mega environment of North western Ethiopia as ranked based on distance to the ideal environment. The double arrow blue line is the average environment coordinate (AEC) and the single arrow red line is the average environment axes (AEA). E1= Debre-Tabor low N(Rainfed), E2= Debre-Tabor high N(Rainfed), E3= Injibara low N (Rainfed), E4=Injibara high N (Rainfed),

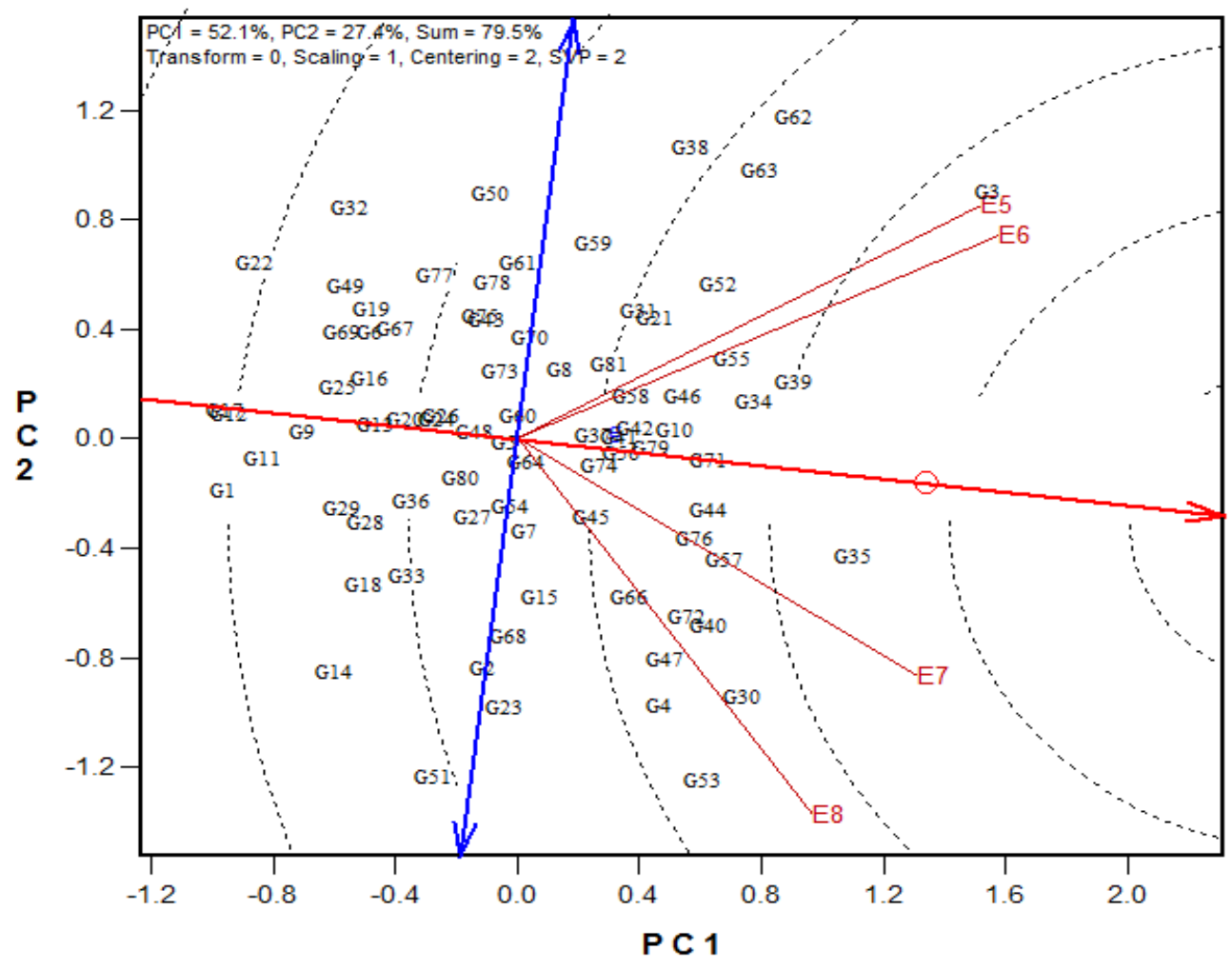

Figure 4. The Representativeness vs Discrimination view of the GGE biplot for test environments based on NUE value of potato cultivars in irrigation mega environment of North western Ethiopia as ranked based on distance to the ideal environment. The double arrow blue line is the average environment coordinate (AEC) and the single arrow red line is the average environment axes (AEA). E5=Injibara low N (irrigation), E6= Injibara high N (irrigation), E7= Koga low N (irrigation), E8=Koga high N (irrigation 
Table 6. Numerical values for the test environments within the rainfed mega-environment based on Figure 3

\begin{tabular}{llll}
\hline Test environment & Vector Length & Correlation with AEA & Distance to ideal test environment \\
\hline E1 & 1.92 & 0.80 & 1.75 \\
E2 & 1.72 & 0.99 & 1.20 \\
E3 & 1.84 & 0.82 & 1.76 \\
E4 & 1.60 & 0.97 & 1.41 \\
\hline
\end{tabular}

E1= Debre-Tabor low N(Rainfed), E2= Debre-Tabor high N(Rainfed), E3= Injibara low N (Rainfed), E4=Injibara high N (Rainfed),

Table 7. Numerical values for the test environments within the irrigation mega-environment based on Figure 4

\begin{tabular}{llll}
\hline Test environment & Vector Length & Correlation with AEA & Distance to ideal test environment \\
\hline E5 & 1.74 & 0.81 & 1.64 \\
E6 & 1.75 & 0.85 & 1.51 \\
E7 & 1.58 & 0.89 & 1.38 \\
E8 & 1.68 & 0.67 & 1.92 \\
\hline
\end{tabular}

E5=Injibara low N (irrigation), E6= Injibara high N (irrigation), E7= Koga low N (irrigation), E8=Koga high N (irrigation

\section{Genotype evaluation}

To identify widely adapted genotypes, the Mean vs. Instability forms of the GGE biplot containing all test environments within each mega-environment are presented in Figure 5 and 6, supplemented with numerical outputs in Supplementary Table 4 and 5. These biplots were constructed based on genotype-focused singular value partitioning to visualize the mean performance and instability of the genotypes. In the biplots, the double-arrow blue line pointing outward from the bi-plot origin which passes through the origin perpendicular to the AEA is the AEC. The AEC from the biplot can be used to visualize the mean performance and instability of the genotypes when it is based on genotype-focused singular value partitioning (Figures 5 and 6). Regardless of the direction the two arrows point to higher instability for the genotypes, i.e. greater contribution to GE interaction, and the small circle on the AEA represents the ideal genotype. The ideal genotype is a virtual genotype that is defined to have the highest value in the trials (the longest vector of all genotypes) that is absolutely stable and on the AEA (Yan, 2014). In Figure 5, the genotype G43 (Kuras) has the longest positive projection along the AEA, suggesting that it has the highest mean NUE across the test environments within the mega-environment. The genotype G2 (Agata) has the longest negative projection onto AEA indicating that it had the lowest mean NUE value across the test environments.

According to Yan (2014), if the test environments are placed on both sides of the AEC ordinate, then the G/GE in the data set would be too small for the AEC to be reliably used for 
genotype evaluation. In Figure 5 all test environments are on the same side of the AEC, indicating that the G/GE in this data set is high enough, and that the AEA is meaningful in genotype evaluation. To better visualize the mean performance and instability of the genotypes, a line was drawn from each genotype to the AEA. The length of the line from AEA is a measure for the genotype's instability. The longer the line, the less stable the genotype. Consequently, G43 (Kuras) contributed little to GE and therefore it was stable, while genotypes like G18 (Carlita) and G39 (Jazzy) contributed more to GE and thus were unstable. Both G18 and G39 were unstable, however they were on opposite sides of the AEA indicating their interactions with the environments were in opposite directions, and they were unstable for contrasting interactions with the environments. Closeness to the ideal virtual genotype helps to select the most stable and best performing genotype within the megaenvironment. The distance of the genotypes to the ideal genotype (GGE distances) as well as their rank relative to the ideal genotype are presented in supplementary table 4 . The higher the mean and the smaller the distance of a genotype to the ideal genotype, the more desirable it is. Thus, G43 is the most desirable, while G2 (Agata) is the least desirable. G9 (Asterix) and G27 (Desiree) were the second and the third most desirable genotypes in this mega environment.

In the same fashion, in the irrigation mega environment (Figure 6), the genotype G3 (Agria) has the longest positive projection, suggesting that it has the highest mean NUE value across the test environments, while G22 (Cleopatra) has the longest negative projection. Genotype G35 (Hermes) was located closer to AEA, and had less contribution to GE. In the contrary, genotypes, G51 (Marabel), G53 (Marilyn) and G62 (Nicola) contributed more to GE interaction suggesting these are unstable. Based on the criteria of the ideal genotype, G35 was the most desirable genotype as placed closer to AEA and had the longest vector next to G3. 


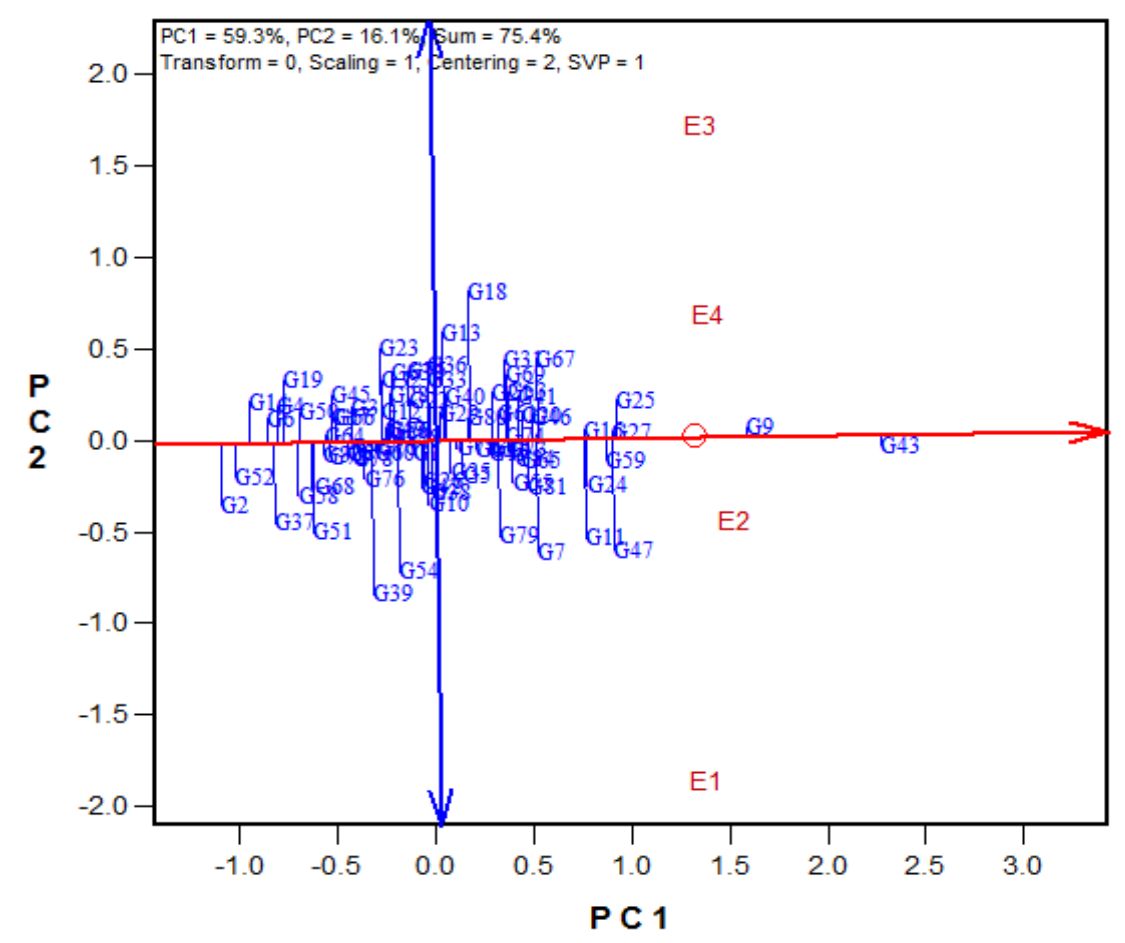

Figure 5. The Mean vs instability view of the GGE biplot for NUE of potato cultivars in rainfed mega environment of North western Ethiopia as ranked based on distance to the ideal genotype. The double arrow blue line is the average environment coordinate (AEC) and the single arrow red line is the average environment axes (AEA)

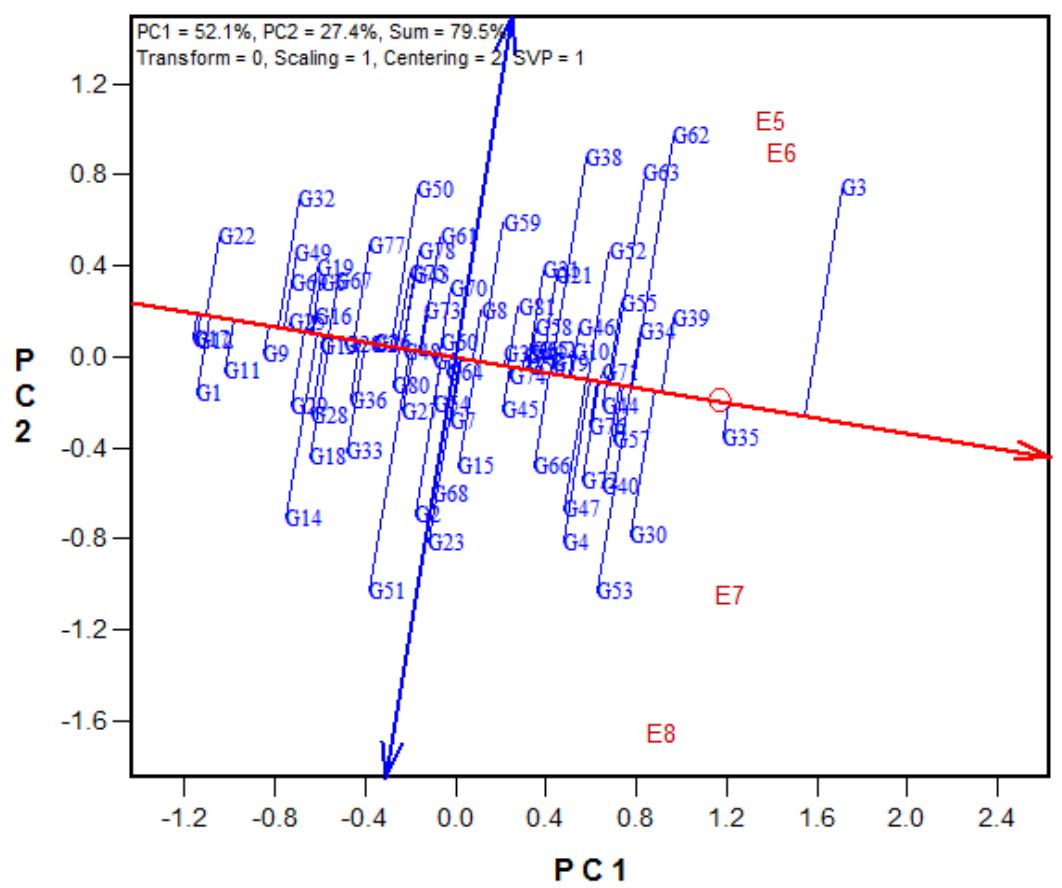

Figure 6. The Mean vs instability view of the GGE biplot for NUE of potato cultivars in the irrigation mega environment of North west Ethiopia as ranked based on distance to the ideal genotype. The double arrow blue line is the average environment coordinate (AEC) and the single arrow red line is the average environment axes (AEA) $\mathrm{G}$ with numbers $=$ Genotype codes ( see the name of the genotypes in Chapter 5 , supplementary Table 2) 


\section{Discussion}

Analysis of genotype-by-environment interaction for a key trait is an important topic of crop variety trial data analysis. To assess mega-environments, test-environments and genotype aspects in a genotype-by-environment analysis, 81 European commercial potato cultivars (G1 to G81) were evaluated for NUE in eight testing environments (E1 to E8) in North western Ethiopia. The effect of environmental conditions on NUE of potato was highly significant. The results of the field trials demonstrated the impact of environment, and in particular production season on the NUE of potato cultivars. Large contributions of the environment that influenced NUE of genotypes was reported in a number of studies (Bertin and Gallais, 2000; Dawson et al., 2008; Agostini et al., 2010; Liu et al., 2012). Significant GE interaction can result in different ranking of potato cultivars and complicate selection, because measured performance in one environment fails to predict performance in another environment (Baker, 1988). To gain more insight in the influence of environment on potato cultivar performance and to increase selection efficiency, a GGE biplot analysis was done to evaluate test environments and cultivars. From the GGE biplot analysis, usually only the first Interaction Principal Component axes (IPCA 1 and IPCA2) are needed to adequately explain the data, even though other IPCAs that have no significant contribution to explain the biplot may be detected (Gauch and Zobel 1997; Yan et al., 2000). In our study the first two (IPCAs) of the biplot indeed accounted for more than $50 \%$ of the $\mathrm{G} \times \mathrm{E}$ interaction, demonstrating that in the GGE biplots model the first two PCs can be used to explain interpretable patterns of the GE interactions.

Figure $2 \mathrm{~b}$ shows that the radiates of the biplot divided the plot into seven sectors, with four environments all appearing in one sector (left of the biplot), and the remaining four appearing in the right side of the biplot. These two sectors had different high NUE vertex genotypes indicating the presence of crossover GE interaction, and suggesting the test environments should be divided into mega environments (Yan et al., 2007). Understanding and identification of mega environments can result in increased heritability through evaluations in relatively well-defined and predictable target environments (Abdalla et al., 1996). These targets the most promising genotypes for a target trait and improves the efficiency of breeding programs. Our results suggest that there are at least two potato mega environments for potato NUE evaluation in North western Ethiopia, coinciding with the rainfed and irrigation production systems (Fig. 2b). 
The relevance of these two mega environments is supported by the fact that environments did not cluster based on location: Environments E3, 4, 5 and 6 are all in Injibara, but these were divided over the two mega-environments based on production system, demonstrating that season was the main contributor for the GE interaction and the driver for the formation of the two mega environments. Previously, CIP-sourced Ethiopian clones and local cultivars were evaluated under rainfed and irrigation production systems in North Western Ethiopia, but stable cultivars for both rainfed production system and irrigation production system were not found, indicating that an independent selection program is required for each production system (Yigzaw et al., (2008). However, to reach a final conclusion, and to recommend different selection programs for each production system, this result needs to be confirmed in trials over more years, and possibly more locations.

Understanding and selection of suitable test environments is one of the most important factors for the success of any plant breeding program (Yan et al., 2011). For selecting a test environment, the discrimination ability and representativeness of the target environment should be taken into account (Yan, 2002; Xu et al., 2014). In the present study, the two test environments represented by high $\mathrm{N}$ level at both Debre-Tabor and Injibara under rainfed production conditions had good discriminating ability and representativeness of the rainfed mega environment, suggesting these test environments may be good test environments for both low and high $\mathrm{N}$ cultivation conditions. In the irrigation mega-environment however, the low $\mathrm{N}$ testing environments were more discriminating and representative, which may suggest that under irrigation production systems, evaluation and selection of cultivars for NUE improvement may be conducted under low N conditions. According to Murphy et al. (2007), the most efficient way to improve crops yield under low input conditions is indeed to select varieties under low input or stress conditions. In the irrigation mega environment the low and high $\mathrm{N}$ environments at Injibara (E5 and E6) were strongly associated with each other. The analysis of variance at this location also showed non-significant genotype by $\mathrm{N}$ level interaction, which means differences between genotypes are consistent from low $\mathrm{N}$ to high $\mathrm{N}$ environment. This suggests no significant shift in rank order of genotypes with respect to NUE occurred between $\mathrm{N}$ levels for these trials at Injibara. The absence of a significant $\mathrm{N}$ effect may be attributed to the strong acidic nature of the Injibara area soil, as low $\mathrm{pH}$ may affect the availability of $\mathrm{N}$ also under the high $\mathrm{N}$ condiitons. Atlin and Frey (1989) found no genotype by $\mathrm{N}$ level interaction in oats yield, suggesting the possibility of indirect selection 
(i.e selection at low $\mathrm{N}$ or high $\mathrm{N}$ for both high and low $\mathrm{N}$ conditions). However, this may be dependent on the genotypes used, and conclusions should be drawn with care.

Genotype evaluation within a mega environment should consider both mean performance and stability (Yan and Kang, 2003). In our GGE biplot analysis the estimations of yield and stability of genotypes is done by using average environment (tester) coordinate (AEC) methods (Figure 5 and 6) according to Yan (2001). Cultivar Kuras was identified as having consistently high relative NUE performance across the rainfed mega-environment. This implies that this cultivar grows well both in low and high $\mathrm{N}$ rainfed environments, indicating that it is able to adapt to available $\mathrm{N}$ in such a way that yield is always high. Farmers, especially in developing countries, need such type of varieties that can give reasonable yield under stress conditions, and respond to ideal conditions with a yield increment.

However, Kuras was not a good performer for NUE in the irrigation mega environment. Given our results, it is likely that this specific adaptation to mega environments should be taken into account, and specifically adapted genotypes for a mega environment should be selected and cultivated in these environments. Although there is an increase in costs of breeding for specific adaptation relative to a wide adaptation strategy, breeding for specific adaptation tends to imply greater genetic gains. The genetic gains can be derived from exploitation of GE interaction effects via useful adaptive traits (Bidinger et al., 1996), as well as increased heritability of the target trait as a consequence of decreased GE interaction (Kang, 1998). Thus, as the rainfed and irrigation mega environments are independent and distinct, different set of improved materials may be required for each of the megaenvironments.

In conclusion, GE interaction was significant for potato NUE indicating that some environments were better for testing than others. The GGE analysis divided the test environments over two mega-environments. The identification of mega-environments in the North western Ethiopian potato production region and seasons may have various implications. First, it offers the opportunity for the potato breeders to also exploit more targeted adaptation for NUE and related traits to achieve maximum yield and NUE. Second, by focusing in breeding programs on target genotype distribution to a specific mega environment the heritability and the efficiency of testing will be improved. In each of the mega environments, the mean performance and stability analysis identified genotypes that had high mean 
performance and stability for NUE, and these may be used as parents for future NUE improvement in potato.

Overall, we obtained valuable information that suggests independent potato varietal selection programmes are required for each production system in North western Ethiopia, however the results and must be verified by additional multi-year data, to reach conclusive final recommendations. 
Chapter 6

General Discussion 
This thesis addressed the complexity and challenges of breeding for NUE, taking potato as a focus crop and North-western Ethiopia as the target study area. Nitrogen is one of the most important yield-enhancing agricultural inputs. Application of inorganic nitrogen on a large scale is important as it is not possible to provide sufficient organic nitrogen in order to feed the world's population (Smil, 1991). However, the cost of inorganic nitrogen will increase with rising costs of energy required for its production. In various parts of the world, farmers are applying too low levels of inorganic and organic nitrogen as a risk-avoiding and costminimizing strategy for cultivation due to the unavailability or high costs of fertilizer, resulting in low yields and sometimes crop failure (Witcombe et al., 2008). Thus, improving NUE of crop plants is of great importance for sustainable agriculture and food security not only now but also so in the future.

Potato is a prime food security crop for smallholder farmers in the highland parts of Northwestern Ethiopia, where nutrient availability and especially a lack of nitrogen is a major constraint for crop productivity. Smallholder potato farms in this area are often characterized by variable conditions and preferences. In this challenging scenario, breeders have a series of options to work with. Conventionally, breeding practices through indirect selection for physiological and agronomic traits that improve NUE under low $\mathrm{N}$ condition can be used. It can also be studied in an integrated manner by means of quantitative genetic approaches using molecular markers, genomics, and combining physiological and agronomic studies (Gallais and Hirel, 2004).

This chapter highlights the initial efforts made in understanding the genetic and physiological basis of NUE in potato. In doing so, it brings together approaches of physiology and genetics to identify traits related with NUE and locate the genomic regions that genetically underlie the variation for those traits (Chapters 3 and 4). We identified traits important for indirect selection of NUE (Chapter 2), and used a genotype x environment interaction analysis (Chapter 5) to identify $\mathrm{N}$ use efficient genotypes and the best testing environment for NUE evaluation in North-western Ethiopia. The complexity of each of the above aspects needs to be understood in order to effectively address the breeding challenges of NUE. NUE is a term that describes a highly complex, multigenic trait, with various interconnected physiological processes involved and modified by numerous other factors. These include environmental factors affecting both the crop growing process and the availability of $\mathrm{N}$ in the soil (Agostini et al, 2010). Nitrogen use efficiency (NUE) was defined by different 
authors in different ways, depending on the objective of the study and the crop under study. In this study NUE is defined as the tuber yield per unit of nitrogen resource available to the plant. Clear understanding of the main mechanisms and inheritance of NUE is lacking (Basra and Goyal, 2002), and this is partly due to the inherent complexity of NUE, as it is a function of multiple interacting genetic and environmental factors (Dawson et al., 2008). The genetic diversity of the germplasm source involved in this study, the environmental conditions and the genetic diversity and QTL mapping are the central points discussed in this concluding chapter.

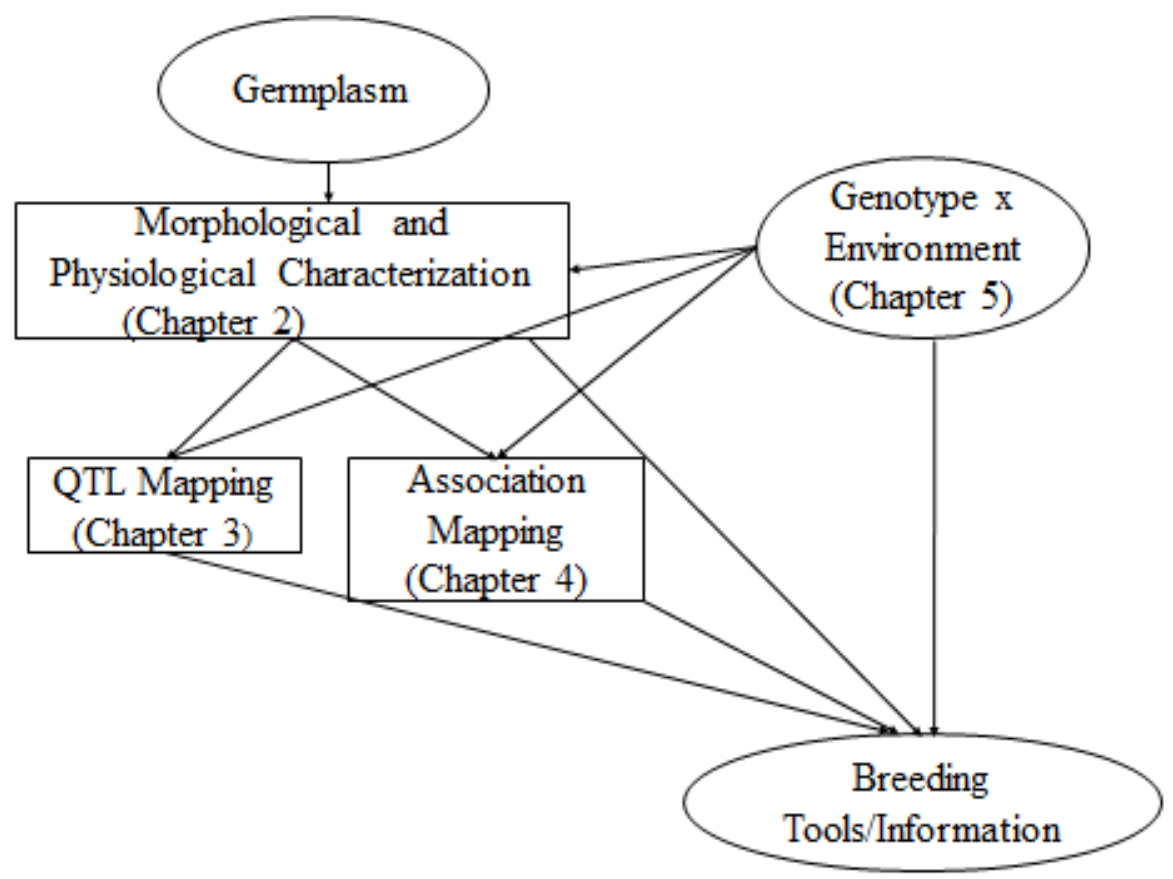

Figure 1. schematic overview of the research presented in this thesis

\section{Germplasm used as a gene source}

Broadening the genetic basis of the gene pool used as a gene source for variety development is highly desirable for any crop, because genetic diversity provides buffering against environmental extremes and biotic stresses. A population with broad genetic variation will have a better chance of surviving and flourishing in a given environment than a population with limited genetic variation. In this thesis, we have used Ethiopian local cultivars, Ethiopian commercial cultivars originating from the International Potato Centre (CIP, Lima Peru) selected under Ethiopian environmental conditions, European commercial cultivars developed 
for European conditions, and the diploid $\mathrm{CxE}$ experimental backcross population to evaluate the genetic variation for NUE under Ethiopian field conditions.

The tested commercial cultivars (both the Dutch and the Ethiopian) in our study showed significant variation that may be used for breeding to improve NUE in potato (Chapter 2, 4 and 5). There is a common understanding that commercial cultivars are not necessarily the most promising source of materials for genetic improvement of NUE, because commercial cultivars may have lost a lot of genetic diversity, as they are mostly developed for high yield under high $\mathrm{N}$ input conditions. Indeed, a wider range of genetic variation in NUE was reported for wild accessions of potato than for commercial potato cultivars (Errebhi et al., 1999). Accessions of $S$. chacoense were identified as a promising source for improving NUE in potato breeding programs (Errebhi et al., 1999). Nevertheless, several studies confirmed that although wild Solanum species may provide a valuable pool of diverse germplasm for this purpose, significant variation in crop $\mathrm{N}$ use efficiency (NUE) is still present in commercial potato cultivars (Midmore et al., 1987; Errebhi et al., 1998b, 1999; Zebarth et al., 2004a; Zvomuya et al., 2002; Sharifi et al., 2007; Ospina et al., 2014). On average, commercial potato cultivars have similar or higher $\mathrm{N}$ use efficiency potential compared to wild potato accessions under both low and high $\mathrm{N}$ supply conditions. However, there were also exceptional wild accessions and/or selections which perform better for some NUE parameters than the commercial cultivars (Errebhi et al. 1998; Zebarth et al., 2008).

The tuber yield mean performance of different cultivar groups in different locations under low and high $\mathrm{N}$ conditions is presented in Figure 2. The maximum mean tuber yield was recorded in Ethiopian local cultivar groups and Dutch commercial cultivar groups at Debre-Tabor and Injibara under low and high $\mathrm{N}$ availability. At low $\mathrm{N}$ there was no clear difference between cultivar groups in both locations. At high $\mathrm{N}$ there was a big difference between the Ethiopian and Dutch cultivar groups in Debre-Tabbor. In Injibara however, the difference between cultivar groups in tuber yield was hardly noticeable, even though the Dutch cultivar group had the highest mean compared to Ethiopian cultivars.

The effect of $\mathrm{N}$ level on the mean tuber yield of each cultivar group was substantial. The yield difference between low $\mathrm{N}$ and high $\mathrm{N}$ levels of the Ethiopian local cultivars group in particular was considerable, indicating their phenotypic plasticity (the potential of the local cultivars to adapt to the different conditions in different environments). Farmers especially in 
developing countries, need such type of varieties that can give reasonable yield under stress conditions, and respond to ideal conditions with yield increment.
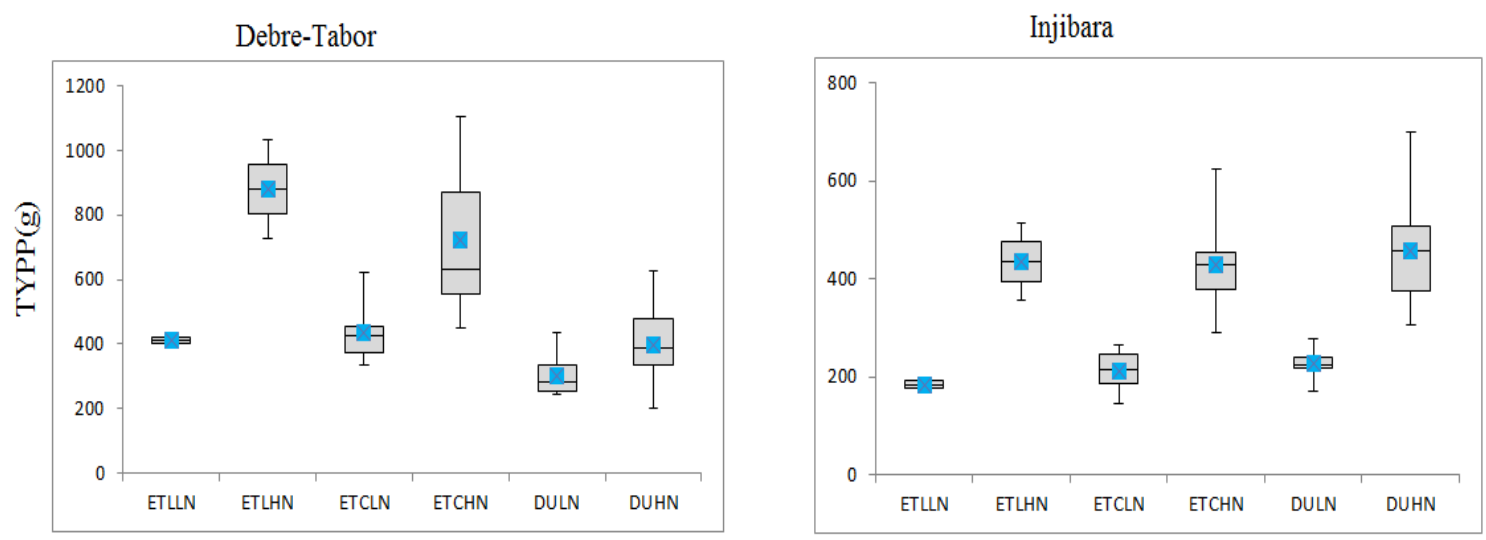

Figure 2. Box plots of tuber yield per plant of potato cultivars in their cultivar group at different nitrogen levels in Debre-Tabor and Injibara in the 2013 rainfed production season. The grouping elements on the $\mathrm{x}$-axis are a combination of $\mathrm{N}$ levels and cultivar groups. ETLLN= Ethiopian local cultivars at low nitrogen, ETLHN= Ethiopian local cultivars at high nitrogen, ETCLN= Ethiopian commercial cultivars at low nitrogen, ETCHN= Ethiopian commercial cultivars at high nitrogen, DULN= Dutch cultivars at low nitrogen, DUHN= Dutch cultivars at low nitrogen;TYPP = tuber yield per plant in $\mathrm{g}$

Overall, commercial cultivars had higher yields than local cultivars, resulting in higher NUE. The local potato cultivars used in this study performed almost similar to the Ethiopian commercial cultivars, and better than most European potato cultivars in NUE under both low and high $\mathrm{N}$ levels, indicating the potential of these genotypes as a genetic resource for NUE improvement programs (Chapter 2). These materials are still in the hands of the local farmers and were cultivated under low input production systems for a long time, and they are potentially more resilient to changes in environmental conditions. Even though there are improved potato varieties developed under high input conditions in Ethiopia, most farmers still prefer to cultivate local cultivars that have higher farm level resilience (Labarta et al., 2012; Kolech et al., 2015). About 70-90\% of the Ethiopian potato farmers are growing at least two local potato cultivars, and $77 \%$ of the total potato growing area in the country is planted with local cultivars every year (Kolech et al., 2015). Especially their potential to give yield without any external application of inorganic fertilizer (only by using the nutrients available in the soil) is of great interest. Foulkes et al. (1998) reported that old cultivars were more able to capture soil nitrogen, while modern cultivars were more able to utilize the high levels of fertilizer nitrogen. According to Kidane Mariam (1979), the Ethiopian local potato cultivars may have originated from a small number of introductions and they are relatively poor in yield. Because of the perception of poor yield, local cultivars have gotten little attention from 
the research community. Instead, varietal development has focused on clones developed by outside sources, primarily the International Potato Center, CIP. According to Williams et al. (1991), the first step in crop improvement for a developing country should be full assessment of local materials. Similarly, Ortiz (2001) reported that including locally adapted potato germplasm in a crossing program would help to ensure the resulting cultivars could be produced in a sustainable and environmentally-friendly manner. Thus, we suggest that the Ethiopian potato breeding program should reconsider local potato cultivars as gene source, either to use them as a cultivar or a parent to cross with commercial potato cultivars for abiotic stress improvement programs in potato. For instance, one of the of Ethiopian traditional cultivars "Agerie" was distinct mainly for its high number of tubers and late maturing characteristics (Chapter 2). These two traits had a strong positive correlation with NUE, indicating that using Agerie in crossing programs can give a pronounced contribution for NUE improvement in potato. In general, to exploit the available genetic resources of potato for NUE improvement of the crop, one should consider local cultivars or landraces, wild potato accessions and other elite materials, which may have a gene source that can be used for potato NUE improvement program.

\section{Environmental conditions for NUE evaluation}

Environmental factors such as rainfall, temperature, light and soil composition vary between locations and growing seasons and can impact NUE of genotypes. In this study, field experiments were conducted in different locations, and under rainfed and irrigation production systems. The objective of the experiments was to evaluate potato genotypes at varying field conditions under low and high $\mathrm{N}$ levels. The study gives an informative overview of performance of cultivars under the various conditions that are relevant for potato cultivation, but that complicate genetic analysis for NUE. This section further compares genetic variation for NUE based on the comparison between: a) Production systems, b) locations, c) $\mathrm{N}$ levels.

\section{Rainfed versus irrigation production systems}

In Ethiopia, two production systems can be distinguished for potato: rainfed and irrigated. The rainfed production system is the dominant production system in which the most of the food crops are grown, and this production system is practised in the rainy season (from May to September). Irrigation production is practiced in the dry season of the year (from November to April). Potato is cultivated in both production systems. However, the crop has 
different production constraints depending on the production system. Higher maximum and lower minimum average temperature are the major irrigated potato production system constraints, while late blight strongly affects potato cultivation in the rainfed potato production system (Yigzaw et al., 2008). According to these authors, cultivars that are optimal for both production systems have not been found as yet.

Our GxE analysis in Chapter 5 indeed indicated that the two production systems are independent mega-environments. As shown in Chapter 5 (Figure 2a and 2b), all irrigation production system test environments were clustered on one side of the biplot while all rainfed production system test environments were on the other side of the biplot. Each cluster had a different highest yielding genotype: cultivar Kuras in the rainfed mega-environment, and Agria in the irrigation mega environment, suggesting that test environments should be divided into mega-environments (Yan et al., 2007). The average performance values of our cultivars were 18 tons/ha for tuber yield and 27 for NUE in the rainfed mega-environment. In the irrigation mega-environment, the genotypes average performance values were 27 tons/ha for tuber yield and 52 for NUE. This low performance of tuber yield and NUE in the rainfed mega-environment compared to irrigation may be related with agro-climatic factors, especially the rainfall (amount and frequency) and temperature.

In North-western Ethiopia, the amount and distribution of rainfall in the rainfed production season can be characterized as erratic (a week or two of heavy rain sandwiched between weeks dry- spilled) in the onset and offset of the season; heavy and frequent in the middle of the season especially around July and August. The relative humidity is also high from July to August. While the irrigation production season is characterized by lower minimum average temperatures (Oct-Dec) and high maximum average temperatures (February to April) as presented in Figure 3a. 

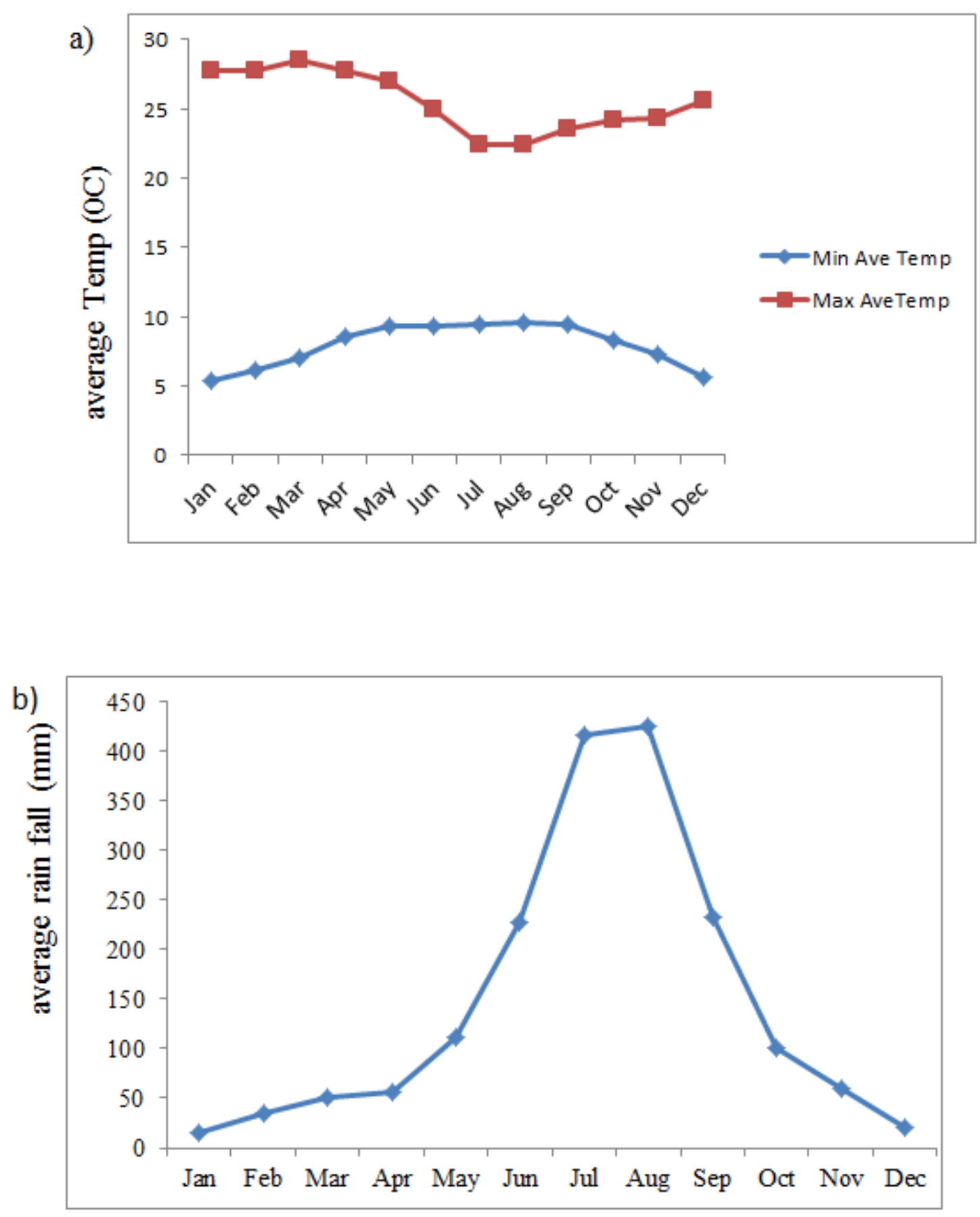

Figure 3. The average minimum and maximum monthly temperatures and average rainfall (2005-2015) of the rainfed and irrigation production seasons in North Western Ethiopia a) Aver.min and max temperature b) average rain fall. The rainfed production season ranges from May to September, and the irrigation production season ranges form November to April (Source: (FAO) local climate estimator online data (New LocClim)). 
As shown in Figure 3a, the average maximum temperature from May to June is $26{ }^{0} \mathrm{C}$, which is good for sprouting and consequently plays a vital role in obtaining high yields in potato. Ahmed (1980) and Zemba et al. (2013) in their study on potato response for some climate variables in Nigeria reported that day temperature ranges of $21-26^{0} \mathrm{C}$ are required for proper sprouting and emergence of the potato tuber. A shift from the upper mentioned range of temperature to higher temperatures at sprouting to emergence/vegetative stage may induce knobbiness and secondary growth in tuber and consequently affect the tuber yield negatively (Ahmed,1980).

As shown in Figure 3b, at the onset of the rainfed production season (May to Mid- June) the amount of rain may be enough for the emergence and early stage growth of potato, however the distribution is erratic. This erratic distribution results in low in soil moisture, which negatively affects the sprouting and emergence of the tuber and finally the ultimate yield. The crop potato requires more frequent supply of water than most other root and tuber crops (Onwoume et al., 1994). The available moisture as well as nutrients in the soil are important for the plant at sprouting to emergence stage of potato development (Burton, 1989). In addition, drought stress studies on different potato varieties revealed that the rate of leaf expansion in the plant was slowed down or ceased and leaf variation was reduced due to water deficit which adversely affect the development of the crop (Sale, 1973; Zaag and Burton 1978; Wolfe et al., 1983).

From end of June to mid-July, the rainfall follows a more regular pattern in North western Ethiopia, which is useful for potato tuber set/initiation. Precipitation is important and significantly positively correlated with tuber set/ initiation stage of potato (Levi, 1999; Eliot, 2007). Early July to mid-August is the time when the average maximum and minimum temperature is around $22{ }^{0} \mathrm{C}$ and $9{ }^{\circ} \mathrm{C}$, respectively (Figure 3a). This temperature is an ideal temperature for tuber bulking. A minimum temperature of $15{ }^{0} \mathrm{C}$ at tuber bulking stage is positively correlated with high tuber yield in potato (Lopez et al,1987; Kochalar, 1991; Levi, 1999; Eliot, 2007; Zemba et al., 2013), and Ochigbo (1993) reported that low temperature is more conducive for tuber growth at bulking, and economic tuber production happens when the average temperature falls below $15{ }^{0} \mathrm{C}$. However, early July to mid-August is the time where frequent and maximum rain fall was recorded in our experiment. When the maximum rainfall coincides with tuber bulking time, yield may reduce, because rainfall increases the threats of potato diseases and insect pest occurrence will also increase. The occurrence and 
severity of late blight (Phytophthora infestans) particularly high when it is accompanied by high relative humidity, dew and frequent rainfall (Hienfling, 1987), a common feature of July and August in North western Ethiopia. Moreover, the soil may become over-saturated, resulting in poor soil aeration and hypoxia.

In the irrigation production season, temperature is the major production constraint of potato. As shown in Figure 3a, in the beginning of the season (October-January) the temperature is low, on average about $7{ }^{0} \mathrm{C}$. This low temperature may cause frost in some years. However, in normal years, if the crop is planted early and reaches maturity in these cold months of the year the low temperature may favour tuber bulking. Ifenkwe and Okonkwo (1983) reported that under irrigation potato production planting should be adjusted to make sure that the time of tuber bulking coincides with the period of low temperature. However, most of the times in North western Ethiopia irrigation is started after the harvest of the rainfed production season crops and the tuberization of potato coincide with the hottest months (Feb-Apr) of the year which may affect tuber initiation as well as bulking negatively. Temperature and photoperiod are the two most important environmental factors that determine potato tuberization. In our irrigation production season, the day temperature was high $\left(25-30{ }^{0} \mathrm{C}\right)$ during tuber initiation and bulking, but tuberization was not inhibited by the high day temperature. This is probably linked to the low night temperature. As shown in Figure 3a, the average minimum temperature during the night was below $10{ }^{0} \mathrm{C}$ throughout the year. The trials in Ethiopia have short day conditions. Short days with cool night temperature favour tuberization, while long days with high temperatures delay or inhibit tuberization (Gregory, 1956; Went, 1959; Slater, 1968).

Overall, in our experiments the average minimum and maximum temperature in the rainfed production season can be considered as good for potato production. The amount and distribution of the rainfall especially at sprouting to emergence stage was also fine, however it was high and frequent at bulking (July to August), due to this reason confounded disease syptoms were observed which may have reduced potato tuber yield. Moreover because of the high intensity of the rainfall, the soil was over-saturated resulting in poor aeration which may have significantly affected the tuber yield in the rainfed prodution season. In the irrigation season experiments, the disease symptoms including late blight were minimal, and the night temperature was also low (on average below $10{ }^{\circ} \mathrm{C}$ ) which favoured the tuberization and the ultimate tuber yield in the irrigation production season. All these climatic differences between 
the rainfed and irrigation production seasons strongly suggest that the two production seasons are distinct, which is strongly reflected in significant genotype-by-environment (GE) interaction (Chapter 5), confirming that the two seasons should be considered as two independent mega-environments and should follow a different selection strategy in breeding.

The Ethiopian potato breeding program developed a number of improved potato varieties and these improved varieties were released only for the rainfed production system under high input conditions. However, farmers are using these rainfed production system varieties for the irrigation production system as well. So far, no clear report has been delivered about the the production area coverage of potato in rainfed and irrigation production season independently. However, the Ethiopian Central Statistics Autority CSA (2014) reported that the total production area coverage of potato was about 179,000 ha with total production of 1.6 million tons. Based on this information the productivity of the crop is about 9 tons/ha which is low compared with the world average productivity of potato (19 tons/ha) (FAOSTAT (2012). Overall, studies on the difference of the two potato production systems is lacking, and improved potato varieties that specifically match requirements for the irrigation production system have not been developed. Although our study may not be conclusive, as megaenvironment analysis require multi-year data, it suggests that the two production systems should be considered as two independent mega-environments for potato tuber yield and NUE improvement evaluation in North western Ethiopia. As presented in Chapter 5, the pooled environment variance component analysis showed low genotypic variance $\left(\sigma^{2} \mathrm{~g}\right)$ compared to estimates of genotype-by-environment interaction variance $\left(\sigma^{2} \mathrm{ge}\right)$ and environmental variance $\left(\sigma^{2} \mathrm{e}\right)$ for NUE, indicating presence of large differences between environments. In particular, the genotype-by-location interaction, which includes production season, had larger contributions to the total genotype-by-environment interaction than the genotype-by-N level interaction. NUE and most-NUE related traits had high genotypic variance and heritability estimates under rainfed production season conditions, whereas under irrigation the estimates were low for most traits suggesting the target breeding environment should be divided into sub-target environments (mega-environments) based on production seasons (Chapter 5), which indicates once more that the Ethiopian potato improvement program should have breeding and selection strategies for both production systems. 


\section{Location}

Location can have a positive or negative impact on the performance of the potato genotypes. The environmental factors that differed most between the locations used in this thesis were altitude, temperature, soil acidity and rainfall. With genotype-by-location two-way data analysis the superiority of tested genotypes in terms of the key traits can be assessed over locations and seasons, or within lcoations and seasons. We have evaluated our cultivars at three different locations: Debre Tabor, Injibara and Koga. The locations can be divided in high altitude (more than 2500 masl) and mid altitude (1900 masl) areas. Debre-Tabor and Injibara are in high altitude areas, but are different in temperature, rainfall and soil $\mathrm{pH}$. Especially the soil of Injibara is more acidic, which is less favourable for crop production compared to Debre-Tabor.

Based on these distinct environmental factors we tried to group our locations using GGE analysis. As shown in Chapter 5 Figure 3, the locations did not cluster in the biplot. Rather, regardless of the locations, our test environments were grouped based on production season. We conducted the experiments at Injibara both in the rainfed and irrigation season, and Injibara as a location was present both in the rainfed and irrigation mega environments. This indicated that the the season effect was larger than the location effect, and that locations may not have to be considered as separate target environments when designing proper strategies of genotype evaluation and cultivar recommendation. However, in the analysis of variance, the test locations within each mega-environment showed significant differences, which indicates that the difference between locations and their appropriateness as a test environment. According to Yan (2014), the test locations within a mega-environment should be different enough from one another to represent the environments that are likely encountered in the mega environment.

In chapter 2, we found a significant difference between the locations (Debre-Tabor and Injibara) in tuber yield. Both Debre-Tabor and Injibara are high altitude areas, and they have similar but not identical environmental indices. The overall $\mathrm{N}$ levels combined average location performance of our cultivars was 18 tons/ha at Debre-Tabor and 15tons/ha at Injibara. The extent of the $\mathrm{N}$ effect on tuber traits was also significantly different between the two locations (Chapter 2). Tuber yield was reduced by $30 \%$ in Debre-Tabor and $48 \%$ in Injibara, and tuber number was reduced by $16 \%$ in Debre-Tabor and $38 \%$ in Injibara due to low $\mathrm{N}$. Both tuber yield difference and effect of $\mathrm{N}$ level on tuber traits may be attributed to soil 
acidity; Injibara is an acidic ( $\mathrm{pH}=4.8)$ area, and this may have had a large contribution to the difference between the two locations. Low pH physiologically impairs the nitrogen cycle and absorption of $\mathrm{Ca}, \mathrm{Mg}$, and $\mathrm{P}$, and increases solubility and toxicity of $\mathrm{Al}, \mathrm{Mn}$ and $\mathrm{Fe}$ (Grime, 2001). Low soil pH also decreases the availability of important macro and micro nutrients, such as Phosphorus, Nitrogen, Calcium, Magnesium, Sulphur, Zinc and Molybednum (Rao et al., 1993). According to Jackson (1967), at low pH autotrophic micro-organisms (Nitromonas and Nitrobacter) which are largely responsible for the nitrification of ammonium to nitrite and nitrite to nitrate both function poorly or not at all. Thus, our locations may be considered as one target mega environment but with sufficient difference between them to use a test locations. However, from the environmental factors acidity may need especial attention in the high land area of North western Ethiopia.

In Ethiopia, about $41 \%$ of the total land area is covered by acid soils, and acid soils with $\mathrm{pH}<$ 5.5 in the surface layer are found in about $13 \%$ of the total acid soil land area (Schlede 1989; Abebe 2007). As a result, the Ethiopian government has made an effort to mitigate the problem through the use of lime on cultivated farm lands especially in the high land parts of the country, where potato is a staple food crop. However, acid soil reclamation through liming requires a large amount of lime. Thus it would be worthy to consider the development of acidtolerant crop varieties for major food crops that are growing in low soil $\mathrm{pH}$ areas. Our result may be used as evidence for the Ethiopian potato breeding program to consider high soil acidity tolerance as a research theme and important trait in future breeding strategies.

\section{Low $\mathbf{N}$ and high $\mathbf{N}$}

Nitrogen use efficiency is affected by the availability of $\mathrm{N}$, and genetic factors determining NUE may be different at high and low $\mathrm{N}$ availability. In maize studies, significant $\mathrm{G} \times \mathrm{N}$ level interaction was found for kernel number (Bertin and Gallais, 2000). In potato, significant $\mathrm{G} x$ $\mathrm{N}$ level interaction was observed for tuber yield, $\mathrm{N}$ utilization efficiency and harvest index (Zebarth et al., 2004b). Lafitte et al. (1997) in their maize landraces NUE study identified different types of groups: some of the cultivars are among the best performers under adequate $\mathrm{N}$ levels, but they are not under limited $\mathrm{N}$ conditions. Other genotypes in the same study showed an opposite response, which indicates specific adaptation to $\mathrm{N}$ environments. This demonstrates that when we conduct a selection for a target trait related to NUE, we will have to consider different $\mathrm{N}$ levels in the growing environments. In studying the efficiency of high $\mathrm{N}$ environments for improving maize for low nitrogen target environments, Bänziger et al. 
(1997) suggested that maize breeding programs targeting low $\mathrm{N}$ environments in the tropics should include low $\mathrm{N}$ selection environments to maximize selection gains.

In Chapter 2, we found significant $\mathrm{G} \times \mathrm{N}$ interaction for NUE and most NUE-related traits, which indicates a genotypic difference in $\mathrm{N}$ responsiveness. The average NUE performance of the population in this study (Chapter 2) was 17 and $35 \mathrm{~kg}$ of dry tuber yield/ $\mathrm{kg}$ of $\mathrm{N}$ respectively under high and low $\mathrm{N}$ conditions. Some of the cultivars that are poor in NUE (below the population average) under high $\mathrm{N}$ conditions were best performers under low $\mathrm{N}$ condition (see Figure 4). However, most of the cultivars that performed well under high $\mathrm{N}$ were also best under low $\mathrm{N}$ conditions. For example, one of the Ethiopian commercial cultivars (Zengena) performed well with an NUE value of $36 \mathrm{~kg}$ of dry tuber yield/ $\mathrm{kg}$ of $\mathrm{N}$ under high $\mathrm{N}$ and 45 under low $\mathrm{N}$ conditions. This cultivar belongs to a group of cultivars that suggests the possibility of selection under high $\mathrm{N}$ environment for a low $\mathrm{N}$ target environment. The GGE biplot analysis in Chapter 5 (Figure 3 in that chapter) also suggests that high $\mathrm{N}$ level test environments at both Debre-Tabor and Injibara were representative test environments for rainfed mega environment.

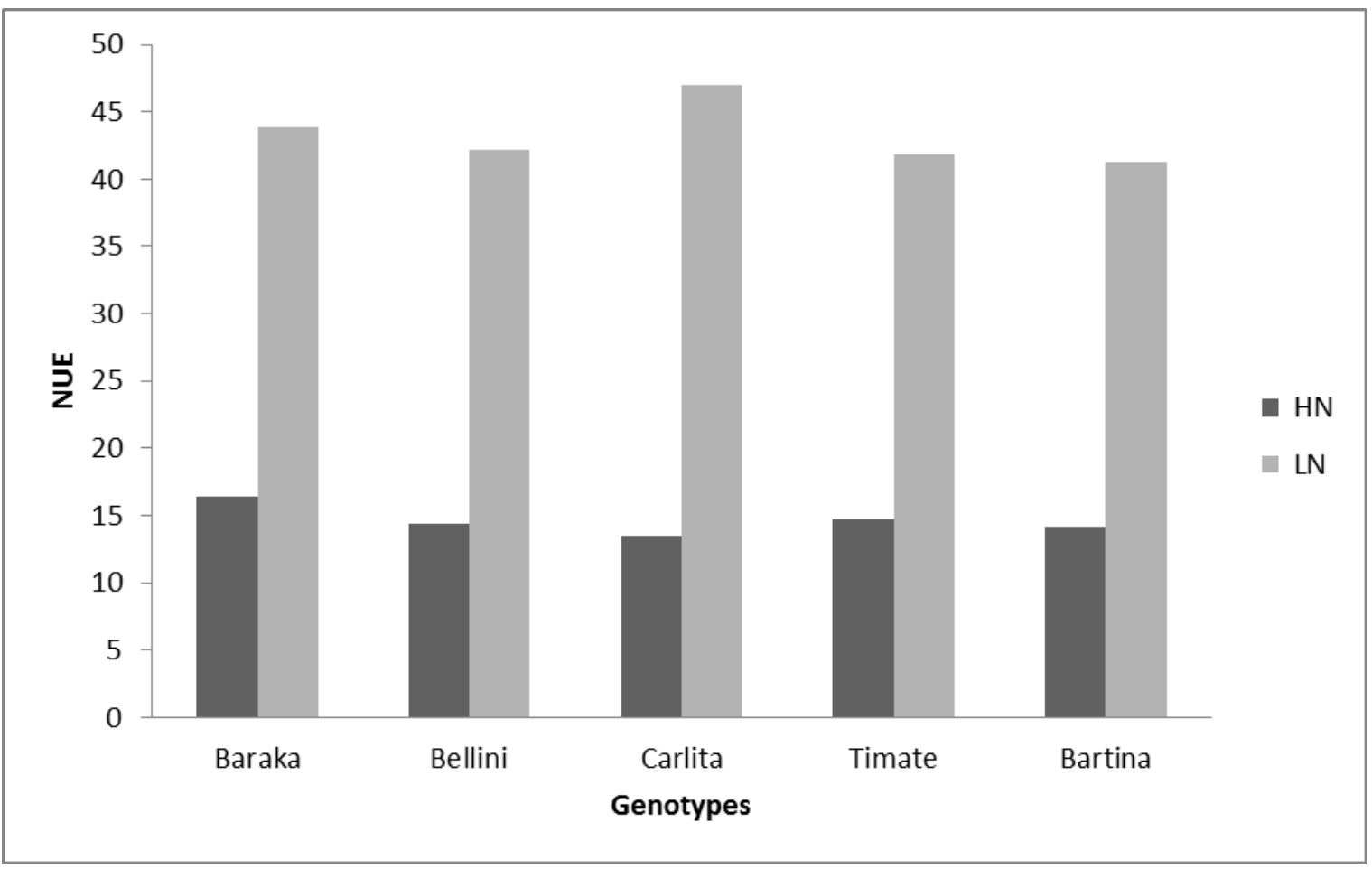

Figure 4. Some selected potato cultivars that showed poor performance under high $\mathrm{N}$ condition and good performance under low $\mathrm{N}$ condition at Debre-Tabor and Injibara (2013). HN = High N conditions $\left(120 \mathrm{~kg} \mathrm{ha}^{-1}\right)$, $\mathrm{LN}=$ Low $\mathrm{N}$ conditions $\left(40 \mathrm{~kg} \mathrm{ha}^{-1}\right.$. NUE = Nitrogen use efficiency $\left(\mathrm{kg} \mathrm{kg}^{-1}\right)$ ' 
Selections for most breeding programs targeting low input production systems are conducted under high $\mathrm{N}$ conditions. However, there is a question as to whether indirect selection under high $\mathrm{N}$ is more efficient than under low $\mathrm{N}$ conditions to improve a target trait under low $\mathrm{N}$ culitvation (target environment). The relative gains of indirect vs direct selection, considering equal selection intensities depends on the estimates of heritability at both $\mathrm{N}$ levels and the genetic correlation between input levels (Falconer, 1974). In maize, (Bänziger et al., 1997; Bertin and Gallais, 2000), in barley (Sinebo et al., 2002) and in wheat, ( Calhoun et al., 1994) estimates of heritability were generally lower under low input level or stressed environments than under high input levels or non-stressed environments. However, Agrama et al. (1999) in their maize NUE study reported heritabilities that were higher at low $\mathrm{N}$ levels than at high $\mathrm{N}$ levels. The estimates of heritability depend on the experimental conditions, so if we manage our experiments precisely we can have high heritability estimates under low $\mathrm{N}$, and selection for low $\mathrm{N}$ production system can be carried out directly under low $\mathrm{N}$ condition.

The level of genetic correlation between environments can greatly differ, depending on the traits studied, the genetic material, and the type of stress as well as its intensity. Atlin and Frey (1989a) found a very high genetic correlation between high and low $\mathrm{N}$ level environments for grain yield of oat lines resulting in similar predictive responses of grain yield to selection in either environment. The genetic correlation between grain yield of maize under low and high $\mathrm{N}$ levels decreased with increasing $\mathrm{N}$ stress intensity (Bänziger et al., 1997), which indicates direct selection in the target environment under more severe stress conditions may be more efficient than indirect selection. Atlin and Frey (1990), and ZavalaGarcia et al. (1992) compared predicted responses of maize grain yield to indirect and direct selection to assess the value of well-watered selection environments for improving grain yield in drought stress environments. They concluded that although estimates of heritability for yield were often lower under stress conditions, direct selection was often superior to indirect selection in targeting yield improvement under stress environment.

In Ethiopia, potato is often grown under low $\mathrm{N}$ conditions because of the cost of fertilizer and limitation of other sources. Potato breeding in Ethiopia is nevertheless conducted under well fertilized (high $\mathrm{N}$ ) conditions, raising the question whether direct selection under low $\mathrm{N}$ should at least be included to improve tuber yield in low $\mathrm{N}$ target environments or the relative contribution of this indirect selection (selection under high $\mathrm{N}$ fertilizer level) to selection gains for our low input production system should be assessed. In our study the correlation 
coefficient and heritability estimate of NUE was similar under low and high N conditions. Tuber yield showed similar correlation and heritability estimates under both $\mathrm{N}$ conditions (Chapter 2 and 3) indicating that direct selection under low $\mathrm{N}$ conditions for low $\mathrm{N}$ target environment is indeed possible.

\section{Genetic diversity and QTL mapping Morphological characterization}

The first step in many breeding projects is to define the desired phenotype and select important traits that can contribute to the improvement of your trait of interest. In addressing the improvement of NUE by evaluation of agro-morphological traits, one needs to determine the level of genetic variation in the different genotypes of a crop and identify the association of other traits with NUE that can contribute to its improvement. According to Gopal (1999), genetic parameters and trait associations provide information about the expected response of different traits to selection and help in developing an optimal breeding strategy. Therefore, to explore the genetic diversity for NUE of cultivated potato from Ethiopia and Western Europe in Ethiopian growing conditions, we addressed the following questions: 1) how much of the total phenotypic variation of traits is due to heritable and non-heritable components, and 2) which agronomic and physiological traits contribute to NUE?

In this study, high heritability estimate differences were observed between Debere-Tabor and Injibara for NUE. The heritability estimates of NUE were 0.8 and 0.72 under low and high N, respectively at Debere-Tabor, and 0.4 at Injibara under both $\mathrm{N}$ levels (Chapter 2). This heritability estimate difference between the two locations for NUE indicates the sensitivity of the trait to environmental differences, possibly linked to its complexity. Thus, it may be challenging to improve such a complex trait by direct selection. Due to this fact, breeders may adopt indirect selection for complex quantitative traits with low heritability, and selection through correlated traits (Hamawaki et al., 2012). Correlation analysis allows to evaluate the degree of association between two traits and the feasibility of indirect selection, which in some cases may lead to faster progress than direct selection.

Strong phenotypic correlation coefficients were observed between NUE and most traits considered in this study across locations (Chapter 2 Table 4). According to Blum (1988) the efficiency of selection for yield under low $\mathrm{N}$ environments may be improved through selection for correlated secondary traits. Thus, the high heritability value of the NUE related traits and their high correlation value with NUE across locations indicate the feasibility of 
these traits for indirect selection for NUE across locations. However, estimates of genetic parameters including heritability depends on the population and the environment. Heritability estimates should refer to defined populations of genotypes and environments (Dudely and Moll, 1969; Nyquist, 1991). Defining the environments and sufficient sampling of the genotypes in a reference population is important because this gives the context to which the heritability estimate refers. Our results may suggest feasibility of the NUE related traits for indirect selection of NUE in the specified locations, however to use this result as breeding strategy for NUE improvement in North western Ethiopia as a target area, heritability estimates should be made from data collected from multiple locations and years representing the target set of environments, or else the estimates will be biased (unless genotype-byenvironment is negligible) (Nyquist, 1991).

\section{QTL analysis}

A major goal in breeding is to understand the genetic basis of variation for quantitative traits and how these interact with the environment; genes that control the quantitative traits may not function similarly in different environments. Consequently, genotypes in various environments could respond differently to environmental changes. This Genotype by Environment interaction is a common feature for quantitative traits and has been a theme of great concern for breeding programs (Falconer, 1981; Lin et al., 1986; Westcott, 1986). With the help of molecular mapping and suitable experimental designs, GE interaction can be dissected into components of QTL-by-Environment Interaction (QEI), which is helpful for marker assisted selection in crop improvement programs, and may allow the design of a cultivar with an optimal combination of genes (alleles) for a given target environment. Numerous cases of QEI have been documented in QTL mapping studies (Bochet et al., 2016; El-Soda et al., 2014; Hai et al., 2008; Pen-Yaun et al., 2006). A large proportion of QTLs identified in these studies showed plasticity in QTL expression, such as between stress and non-stress, and low $\mathrm{N}$ and high $\mathrm{N}$ environments.

In this study, QTLs have been identified in multiple environments using a diploid mapping population and commercial cultivars (Chapter 3 and 4). Most of the identified QTLs and marker-trait associations (MTAs) were environment-dependent. We identified $\mathrm{N}$ levelspecific, production season-specific, and location-specific QTLs. However, some environment-independent QTLs were also identified. Among the identified QTLs in the biparental QTL mapping study (Chapter 3), the QTLs identified for tuber number, maximum 
canopy cover (Vmax), and area under the canopy curve (AUC) colocalized on specific QTL regions in both the rainfed and irrigation production season, suggesting the QTLs were not production season-specific. QTLs identified for tuber yield, NUE and maturity on the other hand were production season- as well as $\mathrm{N}$ level-specific. QE interaction typically reduces the potential of the identified QTLs to be used for marker assisted selection (MAS) across environments. Thus, to utilize our QTLs in breeding programs QE interactions should be taken into account. In this study, the identified QTLs responsible for phenotypic variation of quantitative traits have been categorized into two groups: (i) QTLs that are significant across $\mathrm{N}$ levels and production season environments, (ii) QTLs that show QE interaction, and that are dependent to specific environments $(\mathrm{N}$ level, location, or rainfed or irrigation season dependent). Using the QTLs detected across environments in MAS may have higher yields across $\mathrm{N}$ levels and seasons, but at the expense of optimal yields in each environment: cultivars adaptable to both rainfed and irrigation systems and at both low and high $\mathrm{N}$ levels may have stable yield in all environments, however their yield may be low compared to environment-specific adaptable varieties. Indeed, Finlay et al. (1961) already reported that broadly adaptable varieties may have stable yields in various environments, but at lower level than narrowly adaptable varieties, which perform remarkably well in favourable conditions but poorly in unfavourable ones. MAS using QTLs that are constitutive and that contribute across environments may reduce the cost for multi-environment trials needed to select the experimental materials across environments. MAS using information on plastic QTLs found specifically under low or high $\mathrm{N}$ in each production season enables more efficient selection for high yields under specific conditions, maximizing the yield in that specific production system or for that specific nitrogen input level. Overall, the multi-environment breeding approach that includes various environments often reduces the total response achieved in a specific environment, while environment-specific breeding strategies may increase the cost of the trials. MAS based on the QTL identified in a mega-environment may be an optimal choice for a breeding strategy, reducing costs while considing both stability and mean performance of the genotypes.

Similarly, the QTLs identified in our association mapping (Chapter 4) were production season- as well as N level-specific QTLs for NUE and most NUE related traits, demonstrating the presence of QEI. In this study, we have identified 77 MTAs for 18 agronomic and physiological traits in different environments (Chapter 4 supplementary Table 6). Considering the number of environments and measured traits, the number of detected MTAs were 
relatively few. Regardless of the other factors that could affect the number of detected QTLs, this low MTA detection power was probably related with the size of the experimental population. A relatively small population size significantly decreases the sensitivity of QTL detection (Li et al., 2006). Purcell et al. (2003) in their association genetic studies of complex traits of maize reported that when using a population size of 500 individuals, the probability of identifying a gene that explains $3 \%$ or more of the phenotypic variation of a trait was $80 \%$, while 1500 individuals are required to achieve similar probability of detection power for a gene that explains $1 \%$ of the variation of the targeted traits, which clearly showed the effect of population size on the QTL detection power. In self-pollinated plant species, small population sizes may be adequate for detection of alleles which have a large effect (Rostoks et al., 2006; Atwell et al., 2010). The set we used for association mapping was small, but had little structure and QTLs were detected even with corrections for relatedness and structure. Combined with the fact that QTLs were often not restricted to a single environment and that some QTL regions were supported by other QTL studies, we are confident that QTLs and QTL regions presented in Chapter 4 represent genetic factors contributing to the variation in NUE and NUE-related traits in potato. Nevertheless, the identified QTLs may require further validation with mapping studies using larger populations, and the set of useful QTLs should be extended by using populations that include cultivars selected under low input production systems and the genetic resources available in local varieties, and possibly even wild species.

Overall, NUE is a complex trait, and basic knowledge on how plants respond to different $\mathrm{N}$ regimes and other environmental conditions and use of DNA marker technology is vital to maximize the success rate of potato breeding for nitrogen use efficiency. In the future further emphasis should be placed on the following points:

1) Appropriate germplasm: Successful breeding programs depend on a high level of genetic diversity in breeding materials. In order to broaden the genetic origin of core breeding materials, identification of diverse genotypes for hybridization is vital (Xu et al., 2004; Reif et al., 2005). Up until now, certain national programs may routinely evaluate potato genotypes for NUE that are introduced from other breeding programs for suitability to the country's growing conditions. However, the source of the materials are mostly elite varieties that are under production in different countries, and that have a narrow genetic base. Genotypes selected for their high performance under high input conditions may not be the best cultivars under low input or stress conditions (Murphy et al., 2005). In this study, the Ethiopian local 
cultivars performed better than the Dutch and Ethiopian commercial cultivars in tuber yield under low and high $\mathrm{N}$ conditions especially at Debre-Tabor (Chapter 2). This suggests that the local cultivars are more tolerant to low $\mathrm{N}$ conditions and have better phenotypic plasticity with sufficient $\mathrm{N}$ under Ethiopian climatic conditions.

Overall, cultivars that performed well in canopy cover parameters such as maximum canopy cover (Vmax) and the total area under the canopy cover in the entire crop growing period (AUC) also had relatively high NUE. In an NUE evaluation study with selected wild potato accessions and their hybrids with the haploid USW551 (USW) in low and high $\mathrm{N}$ environments, many of the wild species and crosses were better than commercial cultivars in NUE and biomass accumulation (Errebhi et al., 1999). Among the tested wild species, Solanum chacoense accessions had the highest biomass accumulation and $\mathrm{N}$ uptake efficiency, suggesting wild species like $S$. chacoense can be used as a source of germplasm for NUE improvement in a potato breeding program. To identify the QTL and the genes that regulate these complex traits, high density genetic maps should be constructed with the use of molecular markers. In autogamous crops, constructing genetic map utilizes appropriate mapping populations such as F2, back-cross, double haploids (DH), recombinant inbred lines (RIL) using appropriate parents. However, these are not available to potato, being an outcrossing and self-incompatible crop (Pushkarnath, 1942; Pandey, 1962), and another complicating factor is the tetraploid nature of cultivated potato. Although genetic analysis in a diploid population is easier, potato breeders are forced to utilize the most complex type of diploid mapping population, which is the F1 progeny of two heterozygous parents in which up to four alleles per locus are segregating. However, there are some self-compatible accessions in solanum species like $S$. chacoense that may be utilized ( Cipar et al., 1964). Recently, Endelman and Jansky (2016) developed the first diploid inbred line based F2 population using $S$. chacoense as a male inbred line grandparent, and this species is one of the wild potato species suggested as a germplasm source for NUE improvement in potato (Errebhi et al., 1999). In addition, several efforts are undertaken to introduce hybrid breeding to potato using advanced material that is self-compatible, and they might be successful (Lindhout et al, 2011). Overall, to improve the NUE of potato, the parental selection should consider local landraces, wild potato accessions and other elite materials which may have a known gene source for NUE. 
2) High throughput phenotyping: Accurate and precise phenotyping strategies are important, especially when dissecting the genetic architecture of complex traits into genetic parameters for component traits through QTL mapping or genome wide association studies (GWAS). However, prediction of phenotype from genotype is generally difficult, due to the large number of genes and gene products that contribute to most phenotypes and their interaction with complex and unpredictable environmental influences. According to Myles et al. (2009), one of the challenges to improve a trait regulated by multiple genes is collection of high quality phenotypic data. Despite the fact that plenty of DNA data is available, the implementation of accurate phenotyping for complex traits as part of quantitative or population genetics studies on complex traits remains a major challenge. The selection of germplasm having appropriate levels of relatedness and the generation of high quality phenotype data will be the main determinant to utilize the combined genetic and phenotypic data in the future (Myles et al., 2009). Precise phenotyping is essential to characterize phenotypes in rigorous and formal way, and link these traits to the associated genes and gene variants (alleles). When there is a significant variability in phenotypic scores collected by different individuals, more defined phenotyping protocols are required (Poland and Nelson 2010). The deeper and more detailed the phenotyping can be done, the less complicated the genetic analysis will be, and the higher the chance of detecting QTLs contributing to the variation of the traits.

Recently, image-based high throughput phenotyping platforms have been developed, and the technology was termed plant phenomics (Paproki et al., 2012). Numerous imaging methodologies, such as visible light imaging, infrared imaging, fluorescence imaging, and imaging spectroscopy are being used to extract multi-level phenotype data, from macroscopic to molecular scale (Sozzani et al., 2014). These high throughput phenotyping techniques can be deployed to characterize a large number of individual plants accurately, requiring a fraction of time, cost and labor of the manual techniques (Montes et al., 2007; Furbank, 2009), and enabling the measurement of dynamic traits like plant canopy development traits in relation to NUE. In this thesis, the canopy data for the growth curve models were measured using grid-squares manually. This approach has delived reliable data, but the manual collection is labor-intensive and therefore at relatively low frequency, and we may have lost important information that could have improved our GWAS and QTL analysis.

Efforts have been made to use high throughput phenotyping technology for NUE and related traits. Pavuluri et al. (2015) in their soft red winter wheat field experiment used a proximal 
sensing method (visible light imaging) to evaluate canopy reflectance for the prediction of grain NUE. Ospina (2016), in his NUE study in potato, implemented phenotyping of the canopy cover using a fixed camera above the grid by taking pictures at various growth stages over the crop cycle. This demonstrates that image-based phenotyping methods can be useful in the characterization of complex physiological and agronomic traits related to NUE. It may be hard to use these technologies in developing countries, because of major bottlenecks which include shortage of well-trained personnel and lack of adapted analysis tools. Nevertheless, the emerging virtual platforms assisted by the information and communication technology revolution will help to overcome some of these limitations by providing breeders with better access to phenotyping methods, and robust analytical and data management tools. An example of this is the G4AW project that gives poor farmers in Uganda access to information about their crops from satellite data (http://g4aw.spaceoffice.nl/en/Projects/G4AWprojects/64/Geodata-for-Innovative-Agricultural-Credit-Insurance-Schemes-GIACIS.html)

\section{3) Interaction between NUE and other abiotic stresses:}

Any variance analysis of crop genotypes on yield or NUE will reveal a genetic component, an environmental component and the interaction between these components: $\mathrm{G} \times \mathrm{E}$ interaction. Among environmental factors that can be observed and used to deliver a better understanding of the analysis required to improve NUE, water availability is one of the most important. Water availability affects nutrient transformation of soil-own nutrients into either plantavailable or -unavailable forms (Fierer et al., 2002). It also affects the rate of transformation of fertilizers added to the soil. Subsequently, it affects absorption of nutrients, total nutrient uptake and nutrient composition of plants. Marschner (1986) pointed out that in any case water supply changes resulted in corresponding changes in roots distribution in the soil profile and the amount of nutrient uptake from different layers. Drought conditions also induced root shrinkage and subsequent loss of soil-root contact, as a result it affects nutrient transport to the root surface (Ahmad et al., 2013). Plants grown under water deficit condition may be subjected to water shortage or $\mathrm{N}$ deficiency or a combination of both, and consequently, colimiting the productivity (Sadras, 2005). Nitrogen absorption by crops is reduced under dry conditions, even mineral $\mathrm{N}$ is available in the soil colonized by the roots (Gonzalez-Dugo et al., 2005). Khasanova et al. (2013) also reported that shortage of water decreases growth and physiological functions of the plant, including negative impact on NUE.

Water availability may have been a factor in our trials as well. The tuber yield or NUE performance of our cultivars was low in the rainfed production system compared to irrigation 
production. This low tuber yield or NUE performance in the rainfed production season may be attributed to the erratic rain fall at the onset of the rainfed production season in North western Ethiopia. When the rain fall is erratic, there may be a loss of $\mathrm{N}$ fertilizer through volatilization or changing it into unavailable form, resulted in low tuber yield and NUE performance (Haynes, 1986; Guntinas et al., 2012). In addition, drought spells in between periods of rain may result in limited diffusion of nitrogen to the roots, and uptake and transport of nitrogen in the plant. Moreover, in the rainfed production, the intensity and frequency of rain was high during the tuberization and bulking phase of the potato crop; this high intensity of rain may have enhanced leaching before the applied $\mathrm{N}$ is utilized by the plant.

In addition to drought or availability of water, environmental factors such as temperature and soil type may affect the NUE as they affect either the crop's growing process or the availability of $\mathrm{N}$ in the soil by affecting mineralization of soil organic matter, organic fertilizer and leaching of soil nitrate (Agostini et al, 2010). Liu et al., (2012) reported that NUE could be reduced because of ammonia volatilization with increasing temperature and water limitations in the soil.

Considering the interaction of NUE with other environmental factors, one should identify the major environmental factors that may play a role in the target environment and define their effect on the NUE so as to improve NUE in that target environment. This would require more extensive monitoring of environmental factors in trials, which would have allowed for instance our GxE analysis in Chapter 5 to be extended with location and season-specific temperature influences, or solar radiation data. More data on interaction between plant traits with environmental factors, especially on key traits leading to better adaptation of crops to $\mathrm{N}$ depleted soil under limited water conditions are required to improve the genetics of NUE (Olesen et al., 2011; Piao et al., 2010). In areas where the probability of drought is high, farmers often respond by reducing the amount of $\mathrm{N}$ fertilizer (McCown et al., 1992). In the tropics, most farmers' fields are characterized by more than one abiotic stress, and it would be desirable to increase the tolerance of crops to several stresses that occur in the target environment.

4) Low input breeding strategy: Although there are as many ways of running a breeding programs as there are breeders, most breeding programs both in developed and developing countries share some common concepts and consequently some common ways of handling 
breeding materials. It has been reported that the most efficient way to improve yields under low input conditions is to select crop varieties under low input or stress conditions (Murphy et al., 2007), however this practice is done by few breeding programs ignoring low-input production strategy without suitable cultivars (Fess et al., 2011). Selection is mostly conducted in research stations with optimum amount of fertilizer and management practices (Rathjen \& Pederson, 1986; Atlin and Frey, 1989a, 1989b; Simmonds, 1991), because under these conditions environmental noise can be kept under control, error variance is small and response to selection is high. Other practices, like variety release, and seed distribution systems are common between most breeders in both developed and developing countries. Breeding programs under optimum input and management practices have been very efficient in developed countries, either in favourable environmental conditions or by applying external inputs, under which genotype $\mathrm{x}$ environment interaction is not likely to pose major problems.

However, in a considerable part of the world food crop production is carried out in less than optimal environments that impose stress on the crop. This is especially true for developing countries, where farmers cannot afford optimal fertilizer use and where the risk of crop failure is high mainly due to drought (McCown et al., 1992). Yield levels in fields of these farmers are usually several-fold lower than those attained in research stations, often due to low levels of input (particularly fertilizer), and other environmental factors and management practices that should be considered when developing varieties for these farmers. For example, in Ethiopia farmers started to use commercial fertilizer almost 40 years ago, in 1967 (FAO, 1995), but most of the Ethiopian farmers still cultivate their crop in low input production systems. The amount of fertilizer applied by most Ethiopian farmers is below the recommended level (Mulat 1999), and for instance of the total cereal production areas only $35 \%$ receives chemical fertilizer. The average fertilizer use of Ethiopia is estimated about 17 $\mathrm{kg} / \mathrm{ha}$, which is very low by any standard (Agriculture For Impact, 2014). Considering that this estimate includes all types of nutrients and crops, most of the potato production in Ethiopia is practiced under low $\mathrm{N}$ condition. The main reasons for this low adoption rate of this important agricultural input in developing countries are costs, availability and risk. In Ethiopia, fertilizer availability is not a problem, however, the cost of fertilizer is very high, and therefore most farmers cannot afford to apply optimal amounts of fertilizer. And even if they can afford the cost, they are not confident enough whether they will get profit after covering their production costs because of other production constraints. In Ethiopia, farmers are reluctant to use inorganic fertilizer because their crop may be damaged due to erratic rain 
fall, poor marketing capabilities, high transportation cost, weak extension service, and a lack of credit service (Samuel, 2006), and they will not get a return on their investment in fertilizer.

Yet, the Ethiopian crop breeding strategy in general and the potato breeding program in particular are based on high input production systems, even though the adoption rate by the farmers for potato varieties developed in this system is low in most areas where the new varieties were disseminated (Abebe et al., 2013; Woldegiorgis, 2013). This indicates that there is a mismatch between the goals of the breeders and the preferences of the farmers. As stated before, based on our results, we would recommend cultivars to be developed for two independent mega environments (rainfed and irrigation production system, Chapter 5), as well as under low $\mathrm{N}$ input.

Our research results also indicate that direct selection of genotypes under low $\mathrm{N}$-available conditions is more effective to select $\mathrm{N}$-use-efficient genotypes than indirect selection under optimal $\mathrm{N}$ conditions. Our test environment analysis revealed that the low $\mathrm{N}$ environment was the best selection environment for NUE improvement in the irrigation mega environment while high $\mathrm{N}$ conditions were ideal in the rainfed mega-environment. Although our result is based on single year data for each production system and is certainly not conclusive, it may be used as an indicative result for further investigation in potato NUE improvement in North western Ethiopia. To mitigate the production constraints of the majority of the Ethiopian farmers, the Ethiopian potato breeding program in particular, and the Ethiopian crop breeding system at large should consider a low input breeding strategy.

In conclusion, the results presented in this thesis provide valuable information for screening and evaluation of potato for NUE improvement. Important traits useful for indirect selection of NUE were identified by QTL mapping and correlation studies. Chromosomal regions responsible for regulation of NUE and related traits were identified by QTL and association mapping. Further exploration of the data collected in this thesis and more emphasis on specific traits and QTLs will facilitate marker assisted selection and identification of candidate genes that can be exploited in cultivated potato to improve NUE in this valuable crop. 


\section{References}

Abdalla OS, Crossa J, Autrique E, and Declay IH (1996) Relationships among international testing sites of spring durum wheat. Crop Sci. 36:33-40

Abebe GK, Bijman J, Pascucci S, and Omta S (2013) Adoption of improved potato varieties in Ethiopia: the role of agricultural knowledge and innovation system and smallholder farmers' quality assessment. Agr. Syst. 122: 22-32

Abebe M (2007) Nature and management of acid soils in Ethiopia. Addis Ababa: Ethiopian Institute of Agricultural Research

Agostini F, Tei F, Silgram M, Farneselli M, Benincasa P, and Aller MF (2010) Decreasing N leaching in vegetable crops through improvements in $\mathrm{N}$ fertiliser management, In: E.Lichtfouse (ed.) Genetic engineering, bio-fertilisation, soil quality and organic farming. Sustainable Agr. Rev. 4. Pp. 147-200. Springer, Dordrecht, The Netherlands

Agrama HAS, Zacharia AG, Said FB, Tuinstra M (1999) Identification of quantitative trait loci for nitrogen use efficiency in maize. Mol. Breeding 5:187-195. doi:10.1023/A:1009669507144

Agriculture For Impact (2014) No ordinary matter: Conserving, restoring and enhancing Africa's soils. A Montpellier Panel Report. p13

Ahmad R, Waraich EA, Ashraf MY, Ahmad S, and Aziz T (2013) Does nitrogen fertilization enhance drought tolerance in sunflower? J. Plant. Nutr. 37: 942-963

Ahmed SA (1980) Irish potato, production in Bangladesh: in proceeding of third International symposium on potato production for South-East Asia and pacific region held at Badung Indonesia pp 50

Allen EJ, Scott RK (1992) Principles of agronomy and their application in the potato industry. In: Harris PM (ed) The potato crop: the scientific basis for improvement. Chapman and Hall, London, pp: 816-881

Amira JO, Ojo DK, Ariyo OJ, Oduwaye OA, Ayo-Vaughan MA (2013) Relative discriminating powers Of GGE And AMMI Models in the selection of tropical soybean genotypes. Intl. J. Plant Breed Genetics 7 (2) :139-145

Anithakumari AM, Dolstra O, Vosman B, Visser RGF, Gerard van der Linden C (2011) In vitro screening and QTL analysis for drought tolerance in diploid potato). Euphytica 181:357-369. doi: 10.1007/s10681-011-0446-6 
Anithakumari AM, Karaba Nataraja KN, Visser RGF, Gerard van der Linden C (2012) Genetic dissection of drought tolerance and recovery potential by quantitative trait locus mapping of a diploid potato population. Mol. Breeding 30:1413-1429. doi: 10.1007/s11032012-9728-5

Appleford NE, Evans DJ, Lenton JR, Gaskin P, Croker SJ, Devos KM,Phillips AL, Hedden P (2005) Function and transcript analysis of gibberellin biosynthetic enzymes in wheat. Planta $223(3): 568-582$

Atlin GN, and Frey KJ (1989b) Breeding crop varieties for low input agriculture. Am. J. Altern. Agric. 4 :53-58

Atlin GN, and Frey KJ (1989a) Predicting the relative effectiveness of direct versus indirect selection for oat yield in three types of stress environments. Euphytica 44: 137-142 Atlin GN, and Frey KJ (1990) Selecting oat lines for yield in low productivity environments. Crop Sci. 30: 556- 561

Atwell S, Huang YS, Vilhjálmsson BJ, Willems G, Horton M, Li Y, Meng D, Platt A, Tarone AM, Hu TT, Jiang R, Muliyati N W, Zhang X, Amer MA, Baxter I, Brachi B, Chory J, Dean C, Debieu M, de Meaux J, Ecker JR, Faure, J.M. Kniskern, J.D. Jones, T. Michael, A. Nemri, F. Roux, D.E. Salt, C. Tang, M. Todesco, Traw NMB, Weigel D, Marjoram P,. Borevitz JO, Bergelson J, and Nordborg M (2010) Genome-wide association study of 107 phenotypes in Arabidopsis thaliana inbred lines. Nature/Letters 465:627-63. doi:10.1038/ nature08800

Babiker EM, Gordon TC, Chao S, Newcomb M, Rouse MN, Jin Y, Wanyera R, Acevedo M, Brown-Guedira G, Williamson S, Bonman JM (2015) Mapping resistance to the Ug99 race group of the stem rust pathogen in a spring wheat landrace. Theor. Appl. Genet. 128: 605-612 Baker RJ (1988) Tests for crossover genotype-environmental interactions. Can. J. Plant Sci. 68: $405-410$

Bänziger M, Betrán FJ, Lafitte HR (1997) Efficiency of high-nitrogen selection environments for improving Maize for low-nitrogen target Environments. Crop Sci. 37:1103-1109 Bänziger M, Cooper M ( 2001) Breeding for low input conditions and consequences for participatory plant breeding: examples from tropical maize and wheat. Euphytica 122: 503519

Barraclough PB, Howarth JR, Jones J (2010) Nitrogen efficiency of wheat: genotypic and environmental variation and prospects for improvement. Eur. J. Agron. 33:1-11 doi: org/10.1016/j.eja.2010.01.005 
Bártá J, and Bártová V (2008) Patatin, the major protein of potato (Solanum tuberosum L.) tubers, and its occurrence as genotype effect: Processing Versus Table potatoes. Czech J. Food Sci. 26 (5): 347-359

Basra AS, Goyal SS (2002) Mechanisms of improved nitrogen-use efficiency in cereals. In: Kang, M.S. (Ed.), Quantitative genetics, genomics and plant breeding. CABI publishing, pp. 269-288

Baye BG, Ravishankar H, Singh HA (2005) Variability and association of tuber yield and related traits in potato. EJAS 18(1): 103-121

Berloo R, Hutten R, van Eck H, Visser R (2007) An online potato pedigree database resource. Potato Res. 50: 45-57. doi:10.1007/s11540-007-9028-3

Bernard SM, habash DZ (2009) The importance of cytosolic glutamine synthetase in nitrogen assimilation and recycling. New Phytol. 182 (3) : 608-620

Bernard SM, Moller AL, Dionisio G, Kichey T, Jahn TP (2008) Gene expression, cellular localization and function of glutamine synthetase isozymes in wheat (Ttiticum aestivum L). Plant Mol. Biol. 67: 89-105

Bernardo R (2008) Molecular markers and selection for complex traits in plants: learning from the last 20 years. Crop Sci. 48: 1649-1664

Bertin P, and Gallais A (2001) Physiological and genetic basis of nitrogen use efficiency in maize. I. Agro-physiological results. Maydica 45: 53-66.

Bertin P, Gallais A (2000) Genetic variation for nitrogen use efficiency in a set of recombinant maize inbred lines I. Agro physiological results. Maydica 45: 55-66

Bidinger FR, Hammer GL \& Muchow RC (1996) The physiological basis of genotype by environment interaction in crop adaptation. In M. Cooper \& G.L. Hammer, eds. Plant adaptation and crop improvement, p. 329-347. Wallingford, UK, CABI

Billen G, Garnier J and Lassaletta L (2013) The nitrogen cascade from agricultural soils to the sea: modelling $\mathrm{N}$ transfers at regional watershed and global scales. Philos. Trans. R. Soc. Lond B. Biol. Sci. 368 (1621) : 1-13. doi: 10.1098/rstb.2013.0123

Blum A (1988) Plant breeding for stress environments. (CRC Press: Boca Raton, FL) P. 223 Bochet AS, Laperche A, Bissuel-Belaygue C, Baron C, Morice J, Rousseau-Gueutin M, Dheu J-E, George P, Pinochet X, Foubert T, Maes O, Dugué D, Guinot F, Nesi N (2016) Genetic basis of nitrogen use efficiency and yield stability across environments in winter rapeseed. BMC Genetics 17:131-152. doi: 10.1186/s12863-016-0432-z 
Bocianowski J (2013) Epistasis interaction of QTL effects as a genetic parameter influencing estimation of the genetic additive effect. Genet. Mol. Biol. 36 (1): 93-100

Bocianowski J, Krajewski P (2009) Comparison of the genetic additive effect estimators based on phenotypic observations and on molecular marker data. Euphytica 165: 113-122 Bock BR(1984) Efficient use of nitrogen in cropping systems, P.273-294. In: R.D.Hauck(ed.) Nitrogen in crop production. ASA,CSSA, and SSSA, Madison, WI.

Bonierbale MW, Plaisted RL, Tanksley SD (1988) RFLP Maps Based on a Common Set of Clones, Reveal Modes of Chromosomal Evolution in Potato and Tomato. Genetics 120: 10951103

Borlaug N (2007) Sixty-two years of fighting hunger: personal recollections. Euphytica 157: 287-297

Bouchet A-S, Laperche A, Bissuel-Belaygue C, Baron C, Morice J, Rousseau-Gueutin M, Dheu J-E, George P, Xavier Pinochet X, Thomas Foubert T, Maes O, Dugué D, Guinot F, and Nesi N (2016) Genetic basis of nitrogen use efficiency and yield stability across environments in winter rapeseed, BMC Genetics 17: 131 doi:10.1186/s12863-016-0432-Z

Bowman JC (1972) Genotype x Environment interactions. Ann.Gènèt.Sèl.anim. 4: 7-I23

Bradshaw AD (2006) Unravelling phenotypic plasticity-why should we bother? New Phytol. 170 (4) : 644-648

Bradshaw J E (2009) A genetic perspective on yield plateau in potato. Potato J. 36 (3/4): 79 94

Bradshaw J, Hackett C, Pande B, Waugh R, Bryan G (2008) QTL mapping of yield, agronomic and quality traits in tetraploid potato (Solanum tuberosum subsp. tuberosum). Theor. Appl. Genet. 116:193-211

Brancourt-Hulmel M, Heumez E, Pluchard P, Beghin D, Depatureaux C, Giraud A, and Le Gouis J (2005) Indirect versus direct Selection of winter wheat for low-Input or high-input levels. Crop Sci. 45:1427-1431

Bulmer MG (1985) The mathematical theory of quantitative genetics. Clarendon, Oxford.

Burton DL, Zebarth BJ, Gillam KM, and MacLeod JA (2008) Effect of split application of fertilizer nitrogen on N2O emissions from potato. Can. J. Soil Sci. 88 (2): 229-239

Burton GW, and Devane EH (1953) Estimation of heritability in tall Festuca (Festucaarundinacea) from replicated clonal material. Agron. J. 45: 478-481

Burton WG (1989) The potato, veenman and Zonen, Wageningen. Netherlands p. 382 
Calhoun DS, Gebeyehou G, Miranda A, Rajaram S, and van Ginkel M (1994) Choosing evaluation environments to increase wheat grain yield under drought conditions. Crop Sci. 34:673-678.

Carputo D, Barone A. (2005) Ploidy level manipulations in potato through sexual hybridisation. Ann. Appl. Biol. 146 (1) : 71-79. doi: 10.1111/j.1744-7348.2005.04070.x

Cassman KG, Dobermann A, Walters DT (2002) Agro-ecosystems, nitrogen use-efficiency and nitrogen management. Ambio 31(2): 132-140

Cassman KG, Dobermann A, Walters DT, Yang H (2003) Meeting cereal demand while protecting natural resources and improving environmental quality. Annu. Rev. Environ. Resour. 28:315-58

Chaudhary SK (1985) Variability for some quantitative characters in potato. Crop Improvement 12: 179-199.

Cho Y, Jiang WZ, Chin JH, Piao ZP, Cho YG, McCouch SR, Koh HJ (2007) Identified QTLs associated with physilogical nitrogen use efficiency in rice. Mol. Cells 23: 72-79

CIP (International Potato Center) (2012) Potato: Facts and figures (http:// cipotato.org/potato/facts

Cipar MS, Peloquin SJ, Hougas RW (1964) Variability in the expression of selfincompatibility in tuber-bearing diploid Solanum species. Am. Potato J. 41:155-162

Comstock RE, and Moll RH (1963) Genotype-environment interactions. Pp: 164-196. In W.D. Hanson and H.F. Robinson (ed.) Statistical genetics and plant breeding. National Academy of Sciences-National Research Council Publ. 982. NAS-NRC, Washington, DC

Cruz CD, Regazzi AJ, Carneiro PCS (2004) Biometrical models applied to breeding. genético, 3rd (eds), vol 1. Editora UFV, Viçosa, 480p

CSA (Central Statistical Agency) (2008/2009) Agricultural sample survey: Report on area and production of crops, Addis Abeba, Ethiopia, p 126

CSA (Central Statistical Agency) (2014) Agricultural sample survey report on area and production (private peasant holdings in Meher season). Addis Ababa, Ethiopia: Statistical Bulletin, Volume 5

David MB, Drinkwater LE, McIsaac GF (2010) Sources of nitrate yields in the Mississippi River Basin. J. Environ. Qual. 39: 1657-67

Dawson JC, David R. Huggins DR, Stephen S. Jones SS (2008) Characterizing nitrogen use efficiency in natural and agricultural ecosystems to improve the performance of cereal crops 
in low-input and organic agricultural systems. Field Crops Res. 107 (2):89-101. doi: org/10.1016/i.fcr.2008.01.001

de Groot CC, Marcelis, LFM, van den Boogaard R, Kaiser WM, Lambers H (2003) Interaction of nitrogen and phosphorus nutrition in determining growth. Plant Soil 248 (1): $257-268$

den Nijs TPM and Peloquin SJ (1977) Polyploid evolution via 2n gametes. Am. Potato J. 54: $377-386$

Desai, N C, Jaiminis N ( 1997) Genetic variability, heritability and genetic advance for yield in potato. Potato J. 24:52-58

Dethier J-J, Effenberger A (2012) Agriculture and development: A brief review of the literature. ECOSYS-380; No. of Pages 31

Dewey DR, and Lu KH (1959) A correlation and path co-efficient analysis of components of crested wheat grass seed production. Agro n. J. 51:515-518

D'hoop BB, Paulo M, Kowitwanich K, Sengers M, Visser R, van Eck H, van Eeuwijk F (2010) Population structure and linkage disequilibrium unravelled in tetraploid potato. Theor. Appl. Genet.121: 1151-1170

D'hoop BB, Paulo M, Mank R, van Eck H, van Eeuwijk F (2008) Association mapping of quality traits in potato (Solanum tuberosum L.). Euphytica 161(1):47-60. doi:10.1007/s 10681-007-9565-5

D'hoop BB, Keizer PL, Paulo MJ, Visser RGF, van Eeuwijk FA, van Eck HJ (2014) Identification of agronomically important QTL in tetraploid potato cultivars using a markertrait association analysis. Theor. Appl. Genet. 127 (3) : 731-748. doi:10.1007/s00122-0132254-y

Diaz C, Saliba-Colombani V, Loudet O, Belluomo P, Moreau L, Daniel-Vedele F, MorotGaudry J, and Masclaux-Daubresse C (2006) Leaf yellowing and anthocyanin accumulation are two genetically independent strategies in response to nitrogen limitation in Arabidopsis thaliana. Plant Cell Physiol. 47(1): 74-83. doi:10.1093/pcp/pci225

Dilz K (1987) Efficiency of uptake and utilization of fertilizer nitrogen by plants. In: Jenkinson DS, Smith KA (eds) Nitrogen efficiency in agricultural soils. Elsevier, London and New York, pp 1-26

Ding L, Wang KJ, Jiang GM, Biswas DK, Xu H, Li LF, Li YH (2005) Effects of nitrogen deficiency on photosynthetic traits of maize hybrids released in different years. Ann. Bot. 96 (5): 925-930 
Doerge R W (2002) Mapping and analysis of Quantitative Trait Loci in experimental population. Nature reviews/Genetics 3: 45-52. doi:10.1038/nrg703

Dubetz S, Bole JB (1975) Effect of nitrogen, phosphorus, and potassium fertilizers on yield components and specific gravity of potatoes. Am. potato J. 52:399-405.

Dudely JW, and Moll RH (1969) Interpretation and use of estimates of heritability and genetic variance in plant breeding. Crop sci.9(3): 257-262

Ebdon JS, and Gauch HG (2002) Additive main effect and multiplicative interaction analysis of national turfgrass performance trials: I. Interpretation of genotype 3 environment interaction. Crop Sci. 42 (2): 489-496.

Eliot E (2007). Effect of soil temperature at different growth stages Studies on leaf growth. Agro. J. 58: 169-171

El-Soda M, Malosetti M, Zwaan BJ, Koornneef M, and Aarts MGM (2014) Genotype x environment interaction QTL mapping in plants: lessons from Arabidopsis. Trends Plant Sci. 19(6):390-398

El-Soda M, Martin P, Boer MP, Bagheri H, Hanhart CJ, Koornneef M, and Aarts MGM (2014b) Genotype-environment interactions affecting pre-flowering physiological and morphological traits of Brassica rapa grown in two watering regimes. J. Exp. Bot. 65: 697708. doi:10.1093/jxb/ert434

EMA (1988) National Atlas of Ethiopia, Ethiopian mapping Authority (EMA), Addis Ababa. Endelman JB, and Jansky SH (2016) Genetic mapping with an inbred line-derived F2 population in potato. Theor. Appl. Genet. 129:935-943. doi. 10.1007/s00122-016-2673-7

Errebhi M, Rosen CJ, Gupta SC, and Birong DE (1998) Potato yield response and nitrate leaching as influenced by nitrogen management. Agron. J. 90: 10-15

Errebhi M, Rosen CJ, Lauer FI, Martin MW, Bamberg JB, and Birog DE (1998) Screening of exotic potato germplasm for nitrogen up take and biomass production. Am. J. potato Res. 75 (2): 93-100. doi: 10.1007/BF02883883

Errebhi M, Rosen CJ, Lauer FI, Martin MW, Bamberg JB (1999) Evaluation of tuber bearing solanium species for nitrogen use efficiency and biomass partitioning. Am. J. Potato Res. 76: 143-151. doi: 10.1007/BF02853579

Esfahani ST, Shiran B, Balali G (2009) AFLP markers for the assessment of genetic diversity in European and North American potato Varieties cultivated in Iran. CBAB 9: 75-86

Ewing EE (1981) Heat stress and the tuberization stimulus. Am. Potato J. 58: 31-49 
Ewing EE and Struik PC (1992) Tuber formation in potato: Induction, initiation and growth. Hortic. rev 14: 89-198

Falconer DS, and Mackay TFC (1996) Introduction to Quantitative Genetics. $4^{\text {th }}$ edn. Longman, Harlow, UK.

Falconer DS (1981) Introduction to quantitative genetics. $2^{\text {nd }}$ Edition. New York: Longman Press

Falconer DS (1974) Introduction to quantitative genetics: In French. Masson, Paris

FAO ( Food and Agricultural organization) (2014) New -local climate estimator (LocClim) software and global climate database, available at: http://www.fao.org/NR/climpag/pub/en3_051002_en.asp

FAO (1995) Improving soil fertility in Africa. FAO soils bulletin 14, pp 4-12

FAOSTAT (2008) Countries Profile on potato production and utilization. World, Ethiopa http://faostat.fao.org

FAOSTAT (2011) World Profile on potato production and utilization. http://faostat.fao.org FAOSTAT (2012) World Profile on potato production and utilization. http://faostat.fao.org Fess TL, Kotcon JB and Benedito VA (2011) Crop breeding for low input agriculture: A sustainable response to feed a growing world population. Sustainability 3:1742-1772. doi. $10.3390 /$ su3101742

Fierer N, Schimel JP (2002) Effects of drying-rewetting frequency on soil carbon and nitrogen transformations. Soil Biology \& Biochemistry 34: 777-787

Finlay KW, Wilkinson GN (1961) The analysis of adaptation in a plant breeding program. Aust. J. Agric. Res. 14: 743-754

Fischer RA, Byerlee D, Edmeades GO (2009) Can technology deliver on the yield challenge to 2050? FAO expert meeting on how to feed the world in 2050, June 24-26, Rome

Flessa H, Ruser R, Schilling R, Lotfied N, Munch JC, Kaiser EA, and Beese, F (2002) $\mathrm{N}_{2} \mathrm{O}$ and $\mathrm{CH}_{4}$ fluxes in potato fields: automated measurement, management effects and temporal variation. Geoderma 105: 307-325

Fontaine J-X, Ravel C, Pageau K, Heumez E, Dubois F (2009) A quantitative genetic study for elucidating the contribution of glutamine synthetase, glutamate dehydrogenase and other nitrogen-related physiological traits to the agronomic performance of common wheat. Theor. Appl. Genet. 119: 645-62 
Foulkes MJ, Hawkesford MJ, Barraclough PB, Holdswoth MJ, Kerr S, Kightley S, Shewry PR (1998) Identifying traits to improve the nitrogen economy of wheat: recent advances and future prospects. Field Crops Res. 114: 329-342

Fritsche-Neto R, DoVale JC (2012) Breeding for Stress-Tolerance or Resource-Use Efficiency? In: Roberto Fritsche-Neto and Aluízio Borém (Eds) Plant Breeding for Abiotic Stress Tolerance (Pp 13-19) Springer -Heidelberg, Berlin doi:10.1007/978-3-642-30553-5

Furbank RT (2009) Plant phenomics: from gene to form and function. Funct. Plant Biol. 36: $5-6$

Gallais A and Hirel B (2004) An approach to the genetics of nitrogen use efficiency in maize. J. Exp. Bot. 55(396):295-306. doi: 10.1093/jxb/erh006

Gallais A and Coque M (2005) Genetic variation and selection for nitrogen use efficiency in maize: A synthesis. Maydica 50 (3) : 531-547

kernels by nitrogen-15labeling. Crop Sci. 47: 685-693. doi:10.2135/cropsci2006.08.0523

Galloway JN, Cowling E, Seltzinger SP, Socolow RH (2002) Reactive nitrogen: too much of a good thing? Ambio 31(2): 60-63

Gastal F and Lemaire G (2002) N uptake and distribution in crops: an agronomical and ecophysiological perspective. J. Exp. Bot. 53:789-799 doi: 10.1093/jexbot/53.370.789

Gauch HG and Zobel RW (1997) Identifying mega-environments and targeting genotypes. Crop Sci. 37:311-326

Gebhardt C, Ballvora A, Walkemeier B, Oberhagemann P, and Schüler K (2004) Assessing genetic potential in germplasm collections of crop plants bymarker-trait association: a case study for potatoes with quantitative variation of resistance to late blight and maturity type. Mol. Breeding 13: 93-102

Gebhardt C, Ritter E, Debener T, Schachtschabel U, Walkemeier B, Uhrig H and Salamini F (1989) RFLP analysis and linkage mapping in Solanum tuberosum. Theor. Appl. Genet. 78:65-75

Glass ADM (2003) Nitrogen use efficiency of crop plants: physiological constraints upon nitrogen absorption. Crit. Rev. Plant Sci. 22(5) : 453-470.

Gonzalez-Dugo V, Durand JL, Gastal F, Picon-Cochard C (2005) Short-term response of the nitrogen nutrition status of tall fescue and Italian ryegrass swards under water deficit. Aust. J. Agric. Res. 56:1269-1276. 
Good AG, Shrawat AK, and Muench DG (2004) Can less yield more? Is reducing nutrient input into the environment compatible with maintaining crop production? Trends Plant Sci. 9 (12): 597-605. doi:10.1016/j.tplants.2004.10.008

Gopal J (1998) General combining ability and its repeatability in early generations of potato breeding programmes. Potato Res. 41:21-28

Gopal J (1999) Genetic parameters and character association for clonal selection in potato breeding programs. Agronommie 19:531-539

Govindaraj M, Vetriventhan M, and Srinivasan M (2015) Importance of genetic diversity assessment in crop plants and its recent advances: An overview of its analytical perspectives. Genet. Res. Int. 2015: 1-14. doi:10.1155/2015/431487

Gregory LE (1956) Some factors for tuberization in potato. Am. J. Bot. 43(4):281-288

Grime JP (2001) Plant strategies, vegetation process, and ecosystem properties. Reproduce from Hewitt 1952 by permission of the international society of soil science, John Wiley and Sons, London

Grindlay DJ (1997) Towards an explanation of crop nitrogen demand based on the optimisation of leaf nitrogen per unit leaf area. Journal Agricultural Sciences 128: 377-396

Guntinas ME, Trasar-Cepeda C, Leirós MC, Gil-Sotres F (2012) Effects of moisture and temperature on net soil Nitrogen mineralization: A Laboratory Study. Eur. J. Soil Biol. 48: 73-80. doi.org/10.1016/j.ejsobi.2011.07.015

Gupta PK, Rustgi S, and Kulwal PL (2005) Linkage disequilibrium and association studies in higher plants: Present status and future prospects. Plant Mol. Biol. 57:461-485. doi: $10.1007 / \mathrm{s} 11103-005-0257-\mathrm{Z}$

Hai L, Guo H, Wagner C, Xiao S, Friedt W (2008) Genomic regions for yield and yield parameters in Chinese winter wheat (Triticum aestivum L.) genotypes tested under varying environments correspond to QTL in widely different wheat materials. Plant Sci. 175(3): 226232

Hamawaki OT, Sousa LB, Romanato FN, Nogueira APO, Santos Júnior CD, and Polizel AC (2012) Genetic parameters and variability in soybean genotypes. Com. Sci. 3: 76-83

Han M, Okamoto M, Perrin H, Beatty PH, Rothstein SJ, Good AG (2015)The genetics of Nitrogen Use Efficiency in crop plants. Annu. Rev. Genet.49:269-289

Hanneman R, and Peloquin S (1967) Crossability of 24-Chromosome potato hybrids with 48Chromosome Cultivars. Potato Res. 10 (1): 62-73

Haverkort AJ (1990) Ecology of potato cropping systems in relation to latitude and altitude. Agric. Systems 32:251-272 
Haverkort AJ, Uenk D, Veroude H, Van de Waart M (1991) Relationships between ground cover, intercepted solar radiation, leaf area index and infrared reflectance of potato crops. Potato Res. 34 (1):113-121

Hawkes JG (1990) The potato: Evolution, biodiversity and genetic resources. Belhaven Press, London, p. 259

Hawkes JG (1994) Origin of the cultivated potatoes and species relationships. In: Bradshaw JE, Mackay GR (eds): Potato Genetics. CAB International, Wallingford, 3-42

Haynes RJ (1986) The decomposition process: mineralization, immobilization, humus formation and degradation. In: R.1. Haynes (ed.), Mineral Nitrogen in the Plant-Soil System. Academic Press, Orlando. Pp. 52 - 126

Hienfling JW (1987) Late blight of potato. Technical Bulletin, International potato Centre (CIP) $\mathrm{p} 12$

Hill AR (1986) Nitrate and chloride distribution and balance under continuous potato cropping. Agric. Ecosyst. Environ. 15: 267-280

Hirel B, and Lea PJ (2001) Ammonia assimilation. In PJ Lea, J-F Morot-Gaudry, (eds) plant Nitrogen. (Pp 79-99) Springer-verlag, Berlin

Hirel B, Le Gouis J, Bernard M, Perez P, Falque M, Quétier F, Joets J, Montalent P, Rogwoski P, Murigneux A, Charcosset A (2007a) Genomics and Plant Breeding: maize and wheat. In JF Morot-Gaudry, Lea P, Briat JF, (eds.) Functional Plant Genomics, Science Publishers, Enfield (NH), Jersey, Plymouth, pp: 614-635

Hirel B, Le Gouis J, Ney B, Gallais A (2007b) The challenge of improving nitrogen use efficiency in crop plants: towards a more central role for genetic variability and quantitative genetics within integrated approaches. J. Exp. Bot. 58: 2369-87

Hirel B, Tétu T, Lea PJ, and Frédéric Dubois F (2011) Improving nitrogen use efficiency in crops for sustainable agriculture. Sustainability 3: 1452-1485 doi:10.3390/su3091452

Hoang VN, Alauddin M (2010) Assessing the eco-environmental performance of agricultural production in OECD countries: The use of nitrogen flows and balance. Nutr. Cycl. Agro ecosys. 87 (3): 353-36. doi: 10.1007/s10705-010-9343-y

Hodge A, Robinson D, fitter A (2000). Are microorganisms more effective than plants at competing for nitrogen? Trends Plant Sci. 5: 304-308

Ifenkwe OP, and Okonkwo JC (1983) Determination of the most suitable time to plant Irish potato, taking into account the onset of rains. Annual report Umudike, Umuahia 
Ingvarsson PK, Hall D, Tegstrom C (2010) Using association mapping to dissect the genetic basis of complex traits in plants. Brief Funct Genomics 9:157-165

Ingvarsson PK, Street NR (2011) Association genetics of complex traits in plants. New Phytol. 189:909-22. doi: 10.1111/j.1469-8137.2010.03593.x.

Jackson P, Robertson M, Cooper, M and Hammer, GL (1998) The role of physiological understanding in plant breeding: From a breeding perspective. Field Crops Res. 49: $11-37$

Jackson WA (1967) Physiological effects of soil acidity. (in Soil acidity and Liming, (ed). RW Pearson and F Adams, pp.43-124. Agronomy 12, Am. Soc. Agronomy, Madison, U.S. A Jacobsen E (1980) Increase of diploid formation and seed set in 4x X 2x-crosses in potatoes by genetical manipulation of dihaploids and some theoretical consequences. Zeitschrift für Pflanzenzüchtung 85 (2): 110-21

Jansen RC, Nap JP (2001) Genetical genomics: the added value from segregation. Trends Genet. 17(7): 388-391

Jansky SH, Peloquin SJ (2006) Advantages of wild diploid Solanum species over cultivated diploid relatives in potato breeding programs. Genet. Resour. Crop Evol. 53(4): 669-674 doi:10.1007/s10722-004-2949-7

Johanson HW, Robinson HF, and Comstock RE (1955) Genotypic and phenotypic correlations in soybeans and their implication in selection. Agron. J. 47: 477- 483

Jun TH, Van K, Kim MY, Lee SH, and Walker DR (2008) Association analysis using SRR markers to find QTL for seed protein content in soybean. Euphytica 162: 179-191

Kang MS (1998) Using genotype-by-environment interaction for crop cultivar development. Adv. Agron. 62: 199-252

Kang MS (2002) Genotype-environment interaction: Progress and prospects. In: M.S. Kang (Ed.), Quantitative genetics, genomics and plant breeding, CAB International: Wallingford: England, Pp.221-243

Khan MA, Saravia D, Munive S, Lozano F, Farfan E, Eyzaguirre R, Bonierbale M (2014) Multiple QTLs linked to agro-morphological and physiological traits related to drought tolerance in potato. Plant Mol. Biol. Rep. 33:1286-1298. doi: 10.1007/s11105-014-0824-Z

Khan MS (2012) Assessing genetic variation in growth and development of potato. $\mathrm{PhD}$ thesis Wageningen University, Wageningen

Khan MS, Van Eck HJ, Struik PC (2013) Model-based evaluation of maturity type of potato using a diverse set of standard cultivars and a segregating diploid population. Potato Res. 56(2):127-146 
Khasanova A, Jeremy J. James JJ, and Drenovsky RE (2013) Impacts of drought on plant water relations and nitrogen nutrition in dryland perennial grasses. Plant Soil 372(1): 541-552. doi. 10.1007/s11104-013-1747-4

Kidane Mariam H (1979) Preliminary assessment of the responses of potato genotypes in the eastern, southern and central regions of Ethiopia. EJAS 1(1): 41-47

Killick RJ, and Malcolmson Jk (1973) Inheritance in potatoes of field resistance to late blight (phytophetra infestans) Physiol. Plant Patho. 3: 1221-1231

Kleinkopf GE, Westermann DT, Wille MJ, Kleinschmidt GD ( 1987) Specific gravity of Russet Burbank potatoes Am. Potato J. 64: 579-587

Kloosterman B, Abelenda JA, Gomez MDC, Oortwijn M, de Boer JM, Kowitwanich K, (2013) Naturally occurring allele diversity allows potato cultivation in northern latitudes. Nature 495: 246-250. doi:10.1038/nature11912

Kochalar SL (1991) Tropical crops. Macmillan press limited London Pp. 35-40

Kolech SA, Halseth D, De Jong W, Perry K, Wolfe D, Tiruneh FM, and Schulz S (2015) Potato cultivar diversity, determinants and implications for potato breeding strategy in Ethiopia. Am. J. Potato Res. 92: 551-566

Kuchel H, Williams K, Langridge P, Eagles HA, Jefferies SP (2007) Genetic dissection of grain yield in bread wheat. II. QTL-by-environment interaction. Theor. Appl. Genet. 115: $1015-27$

Labarta R, Wambungu S, Yirga C, Mugabo J, Nsabimana J, Mulwa C, Schulz S, Lorochelle C, Alwang J, Andrad R and Yigezu Y (2012) Report on objective 2 of the diffusion and impacts of improved cultivars in Africa (DIIVA) project. Kenya: International Potato Center (CIP), Nairobi

Lafitte HR, and Edmeades GO (1994) Improvement for tolerance to low soil nitrogen in tropical maize III. Variation in yield across environments. Field Crops Res. 39 (1): 27-38

Lafitte HR, Edmeades GO, Taba S (1997) Adaptive strategies identified among tropical maize landraces for nitrogen-limited environments. Field Crops Res. 49: 187-204

Lal GS, Bhaderiya VS, Singh AK (1997) Genetic association and path analysis in elite lines of sunflower. Crop Res. Hisar 13:631-634

Lawlor DW, Lemaire G, Gastal F (2001) Nitrogen, plant growth and crop yield. In: Lea, P.J., Morot-Gaudry, J.-F. (Eds.), Plant Nitrogen. Springer-Verlag, Berlin, pp: 343-367 
Le Gouis J, Beghin D, Heumez E, Pluchard P (2000) Genetic differences for nitrogen uptake and nitrogen utilisation efficiencies in winter wheat. Eur. J. Agron. 12 (3):163-173. doi:

\subsection{6/S1161-0301(00)00045-9}

Lebreton C, Lazic-Jancic V, Steed A, Pekic S, Quarrie S A (1995) Identification of QTL for drought responses in maize and their use in testing causal relationships between traits. J. Exp. Bot. 46 (288): 853-865

Levi D (1999) The responses of potato to a single transient heat or droughts stress imposed at different stages of tuber growth. Potato Res. 28: 415-424

Li Q, Li L, Yang X, Bai G, Warburton ML, Dai J, Li J, and Yan J. (2010) Relationship, evolutionary fate and function of two maize genes orthologous to rice GW2 associated with kernel size and weight. BMC Plant Biol. 10:143-158

Li X, Quigg RJ, Zhou J, Xu S, Masinde G, Mohan S, and Baylin DJ (2006) A critical evaluation of the effect of population size and phenotypic measurement on QTL detection and localization using a large F2 murine mapping population. Genet. Mol. Biol. 29 (1) :166-173 Lin CS, Binns M R, Lefkovitch LP (1986) Stability analysis where do we stand? Crop Sci. 26: 894-900

Lindhout P, Meijer D, Schotte T, Hutten RCB, Visser RGF, van Eck HJ (2011) Towards F1 hybrid seed potato breeding. Potato Res. 54:301-312

Lisinska G, and Liszczynski W (1989) Potato science and technology. Elsevier, New York Liu G, Li Y, Alva AK, Porterfield DM, Dunlop J (2012) Enhancing nitrogen use efficiency of potato and cereal crops by optimizing temperature, moisture, balanced nutrients and oxygen bioavailability. J. Plant nutr. 35 (3):428-441

Lopez DF, Boe AA, Johansen RH, and Jansky SH (1987) Genotype x environment interactions, correlations and coping ability of six traits in potato. Am. potato J. 64:447

Loudet O, Chaillou S, Merigout P, Talbotec J, and Daniel-Vedele F (2003) Quantitative trait loci analysis of nitrogen use efficiency in Arabidopsis. Plant Physiol. 131: 345-358. doi: 10.1104/pp.102.010785

Mahalanobis PC (1936) On the generalized distance in statistics. Proc. Natl. Sci. India B. 2: 49-55

Makino A, Osmond B (1991) Effects of nitrogen nutrition on nitrogen partitioning between chloroplast and mitochondria in pea and wheat. Plant Physiol. 96: 355-362 
Malosetti M, van der Linden CG, Vosman B, van Eeuwijk FA (2007) A mixed-model approach to association mapping using pedigree information with an illustration of resistance to Phytophthora infestans in potato. Genetics 175: 879-889

Maris B (1964) Studies concerning the relationship between plant height of potatoes in the seedling year and maturity in the clonal generations. Euphytica 13 (2):130

Marschner H (1986) Mineral nutrition in higher plants. Wd Ltd. The Greystone Press, Antrim, Northern Ireland

Mather DE, Hayes PM, Cahlmers K, Eglinton J, Mathus I, Richardson K (2004) Use of SSR marker data to study linkage disequilibrium and population structure in Hordeum vulgare: Prospects in association mapping in barley. In linkage disequilibrium workshop, April 4-7, Novotel Barossa Valley Resort, South Australia

McCord PH, Sosinski BR, Haynes KG, Clough ME, and Yencho GC (2011) Linkage mapping and QTL analysis of agronomic traits in tetraploid potato (Solanum tuberosum subsp tuberosum). Crop Sci. 51:771-785. doi:10.2135/cropsci2010.02.0108

McCown RL, Keating BA, Probert ME, \& Jones RK (1992) Strategies for sustainable crop production in semiarid Africa. Outlook on Agric. 21: 21-31.

Meyer C, Stitt M (2001) Nitrate reduction and signalling. In Pj Lea, J-F Morot-Gaudry, eds, plant nitrogen. Springer-verlag, Berlin, pp: 37-59

Mezmouk S, Dubreuil P, Bosio M, Decousset L, Charcosset A, Praud S, and Mangin B (2011) Effect of population structure corrections on the results of association mapping tests in complex maize diversity panels. Theor. Appl. Genet. 122: 1149-1160

Midmore DJ, Vallejo J, Landeo J ( 1987) Selection of nitrogen use efficiency in potato under cool tropical condition. In 10th triennial conference of the European Association for potato research, 26-31 July, Aalborg, Denmark, pp. 243-244

Miralles DJ, Slafer GA (2007) Sink limitations to yield in wheat: How could it be reduced? J. Agric. Sci. 145: 139-149

Moll RH, Kamprath EJ, and Jackson WA (1982) Analysis and Interpretation of Factors Which Contribute to Efficiency of Nitrogen Utilization. Agron. J. 74(3):562-564

Montes JM, Melchinger AE, Reif JC (2007) Novel throughput phenotyping plat-forms in plant genetic studies. Trends Plant Sci. 12: 433-436.

Mostafa K, Fotokian M H, Miransari M (2011) Genetic diversity of wheat (Triticum astivum L.) genotypes based on cluster and principal analysis for breeding strategies. AJCS. 5(1):1724 
Mulat D (1999) The Challenge of increasing Food Production in Ethiopia, In: Alemayehu G and Berhanu N (eds.), The Ethiopian Economy: Performance and Evaluation, Proceedings of the $8^{\text {th }}$ Annual Con. on the Ethio. Economy, Nazareth, Ethiopia

Munoz F, Mylavarapu RS, and Hutchinson CM (2005) Environmentally responsible potato production systems: J. Plant Nutr. 28: 1287-1309

Murphy HJ, and Goven MJ (1959) Nitrogen, spuds and specific gravity. Maine Farm Res. 7 (1): 21-24

Murphy KM, Lammer D, Lyon S, Carter B, Jones SS (2005) Breeding for organic and lowinput farming systems: An evolutionary-participatory breeding method for inbred cereal grains. Renew. Agric. Food Syst. 20: 48-55

Murphy KM, Campbell KG, Lyon SR, Jones SS (2007) Evidence of varietal adaptation to organic farming systems. Field Crop Res. 102: 172-177

Myles S, Peiffer J, Brown PJ, Ersoz ES, Zhang Z, Costich DE, and Buckler ES (2009) Association mapping: Critical considerations shift from genotyping to experimental design. Plant Cell 21:2194-2202. doi:10.1105/tpc.109.068437

Navabi A, Yang RC, Helm J, Spawer DM (2006) Can spring wheat growing megaenvironments in the Northern Great Plain be dissected for representative locations or nicheadapted genotypes? Crop Sci. 46: 1107-1116

Neder DG, Costa FR da, Edvan RL, Filho LT (2013) Correlations and path analysis of morphological and yield traits of cactus pear accessions. Crop Breed. Appl. Biotechnol. 13: 203-207

Neele AEF, Nab HJ, Louwes KK (1991) Identification of superior parents in a potato breeding program. Theor. Appl. Genet. 82:264-272

Neumann K, Kobiljski B, Dencic S, Varshney RK, and Borner A (2011) Genom-wide association mapping: a case study in bread wheat (Triticum aesstivum L) Mol. Breeding 27: $37-58$

Nyquist WE (1991) Estimation of heritability and prediction of selection response in plant populations. Crit. Rev. Plant Sci. 10: 235-322

Obara M, Kajiura M, Fukuta Y, Yano M, Hayashi M, Yamaya T, Sato T (2001) Mapping of QTLs associated with cytosolic glutamine synthetase and NADH-glutamate synthase in rice (Oryza sativa L.) J. Exp. Bot. 52 (359): 1209-17

Ochigbo AA (1993) The production of potato, extension bulleting No.48 Horticultural series No.2 ASRLS. ABU Zaria and NRCRI Umudike pp.1-5. 
Ojala JC, Stark JC, and Kleinkopf GE (1990) Influence of irrigation and nitrogen management on potato yield and quality. Am. Potato J. 67:29-43

Okazawa Y (1967) Physiological studies on the tuberization of potato plants. Journ. Facul. Agri. Hokkaido Univ. Sapporo. 55 (3): 267-336

Olesen JE, Trnka M, Kersebaum KC, Skjelvag AO, Seguin B, Peltonen-Sainio P, Rossi F, Kozyra J, and Micale F (2011) Impacts and adaptation of european crop production systems to climate change. Eur. J. Agron. 34: 96-112

Olsen N, Kleinkopf GE, and Stark JC (2003) Physiological disorders. In stark JC, Loves SL (eds). Potato production systems. University of Idaho Extension, Moscow, pp 309-327

Onwoume I, Charles W (1994) Tropical Root and Tuber Crops. FAO, Rome

Orsel M, Filleur S, Fraisier V, Daniel-Vedele F (2002). Nitrate transport in plants: which gene and which control? J Exp Bot 53: 825-833 doi: 10.1093/jexbot/53.370.825

Ortiz R (2001) The state of the use of potato genetic diversity. Broadening the genetic base of crop production. (Eds). H.D. Cooper, C. Spillane and T. Hodgkin. IPGRI/FAO

Ortiz R, Frusciante L, Carputo D (2005) Dedication: Stanley J. Peloquin potato geneticist and cytogeneticist. Plant Breed Rev. 25: 1-19. doi: 10.1002/9780470650301.ch1

Ospina CA, Lammerts van Bueren ET, Allefs JJHM, Engel B, van der Putten PEL, van der Linden CG, Struik PC (2014) Diversity of crop development traits and nitrogen use efficiency among potato cultivars grown under contrasting nitrogen regimes. Euphytica 199:13-29. doi:10.1007/s10681-014-1203-4

Ospina CA (2016) Nitrogen use efficiency in potato: an integrated, agronomic, physiological and genetic approach. PhD thesis, Wageningen University, Wageningen

Pack JE, Hutchinson CM, and Simonne EH (2006) Evaluation of controlled-release fertilizers for northeast Florida chip potato production. J. Plant Nutr. 29:1301-1313

Pandey KK (1962) Interspecific incompatibility in Solanum species. Am. J. Bot. 49:874-882

Pankhurst R (1964) Notes on the history of Ethiopian Agriculture. Ethio. Observer.7: 210-240 Paproki A, Sirault X, Berry S, Furbank R, and Fripp J(2012) A novel mesh processing based technique for 3D plant analysis. BMC Plant Biol. 12:63-76. doi: 10.1186/1471-2229-12-63

Paul MJ, Foyer CH (2001) Sink regulation of photosynthesis. J. Exp. Bot. 52: 1383-1400

Pavuluri K, Chim BK, Griffey CA, Reiter MS, Balota M, Thomason WE (2015) Canopy spectral reflectance can predict grain nitrogen use efficiency in soft red winter wheat. Precis. Agric. 16 (4):405-424 
Pelleschi S, Leonardi A, Rocher JP, Cornic G, de Vienne D, Thevenot C, Prioul JL (2006) Analysis of relationships between growth, photosynthesis and carbohydrate methabolism using quantitative trait loci (QTL) in young maize plants subjected to water deprivation. Mol. Breeding 17:21-39

Peloquin SJ, Yerk GL, Werner JE, Darmo E (1989) Potato breeding with haploids and 2n gametes. Genome 32: 1000-1004

Pen-Yaun L, Jun Z, Lu Y (2006) Impacts of QTL x Environment interactions on genetic response to marker-assisted selection. Actu. Genetica Sinica 33 (1): 63-71

Peralta JM, and Stockle CO (2002) Dynamics of nitrate leaching under irrigated potato rotation in Washington State: A long-term simulation study. Agric. Ecosys. Environ. 88: 2334.

Piao S, Ciais P, Huang Y, Shen Z, Peng S, Li J, Zhou L, Liu H, Ma Y, Ding Y, Friedlingstein P, Liu C, Tan K, Yu Y, Zhang T, and Fang J (2010) The impacts of climate change on water resources and agriculture in China. Nature 467: 43-51. doi:10.1038/nature09364

Plaisted RL, Sanford L, Federer WT, Kehr AE, Peterson LC (1962) Specified and general combining ability for yield in potatoes. Am. potato J. 39:185.197

Poland J, Nelson R (2010) In the eye of the beholder: the effect of rater variability and different rating scales on QTL mapping. Phytopathology 101(2):290-8. doi: 10.1094/phyto03-10-0087

Presterl T, Seitz G, Landbeck M, Thiemt EM, Schmidt W, Geiger HH (2003) Improving nitrogen use efficiency in European maize: estimation of quantitative genetic parameters. Crop Sci. 43: 1259-1265

Prioul JL, Quarrie SA, cause M, de Vienne D (1997) Dissecting complex physiological functions through the use of molecular quantitative genetics. J. Exp. Bot. 48:1151-1163

Pritchard JK, Stephens M, Rosenberg NA, and Donnelly P (2000) Association mapping in structured populations. Am. J. Hum. Genet. 67: 170-181

Purcell S, Cherny SS, and Sham PC (2003) Genetic power calculator: Design of linkage and association genetic mapping studies of complex traits. Bioinformatics 19: 149-150

Pushkarnath P (1942) Studies on sterility in potatoes. 1. The genetics of self- and crossincompatibilities. Indian J. Genet. Pl. Br. 2:11-36

Quarrie SA (1996) New molecular tools to improve the efficiency of breeding for increased drought resistance. Plant growth regul. 20 (2) :167-178. doi:10.1007/BF00024013 
Rao IM, Zeigler RS, Vera R, Sarkarung S (1993) Selection and breeding for acid-soil tolerance in crops. BioScience 43: 454-465

Rathjen AJ, and Pederson DG (1986) Selecting for improved grain yields in variable environments. In: Plant Breeding Symposium, pp. 104-115. DSIR, Christchurch, N. Z. Agronomy Society Special Publication No . 3

Raun WR, and Johnson GV (1999) Improving nitrogen use efficiency for cereal production. Agron. J. 91(3):357-365

Regassa D, Basavaraj N (2005) Genetic variability studies in potato (Solanum tuberosum L.). Karnataka J. Agric. Sci.18(1):(87-90)

Reif JC, Zhang P, Dreisigacker S, Warburton ML, Ginkel Mvan, Hoising-ton D, Bohn M, Melchinger AE (2005) wheat genetic diversity trends during domestication and breeding. Theor. Appl. Genet. 110(5) 859-864

Remans T, Nacry P, Pervent M, Girin T, Tillard P, Lepetit M, Gojon A (2006) A central role for the nitrate transporter NRT2.1 in the integrated morphological and physiological responses of the root system to nitrogen limitation in Arabidopsis. Plant Physiol. 140, 909921

Richards JH, Milburn PH, MacLean AA, and Demerchant G (1990) Intensive Potato production effects on nitrate- $\mathrm{N}$ concentrations of rural new Brunswick well water. Can. Agr. Eng. 32: 189-196

Roberts S, Weaver WH, Phelps JP (1982) Effect of rate and time of fertilization on nitrogen and yield of Russet Burbank potatoes under center pivot irrigation. Am. Potato J. 59: 77-96

Robson A D, Abbott LK (1989) The effect of soil acidity on microbial activity in Soils. In: A. D. Robson (ed). Soil Acidity and Plant Growth, Academic Press, Sydney, pp: 167-203

Ross H (1986) Potato breeding-problems and perspectives. In: PareyP (ed) Advances in Plant Breeding, Verlag Berlin

Ross-Ibarra J, Morrell PL, and B. S. Gaut BS (2007) Plant domestication, a unique opportunity to identify the genetic basis of adaptation. Proc. Natl. Acad. Sci. U S A. 104 (1) : $8641-8648$

Rostoks N L, Ramsay K, MacKenzie L, Cardle PR, Bhat ML, Roose JT, Svensson N, Stein RK, Varshney DF, Marshall A, Graner TJC, and Waugh R(2006) Recent history of artifi cial outcrossing facilitates whole-genome association mapping in elite inbred crop varieties. Proc. Natl. Acad. Sci. USA 103:18656-18661 
Rosyara U, De Jong W, Douches D, Endelman J (2016) Software for genome-wide association studies in auto-polyploids and its application to potato. Plant genome 9(2): 1-10 Rovaris SRS, de Arau'jo PM, Garbuglio DD, Prete CEC, Zago VS, da Silva LJF (2011) Estimates of genetic parameter in maize commercial variety IPR 114 at Parana' State. Brazil Acta Sci. Agron. 33(4): 621-625

Rowe PR (1969) Nature, distribution and use of diversity in the tuber bearing Solanum species. Economic Bot. 4:331-338

Sadras VO (2005) A quantitative top-down view of interactions between stresses: theory and analysis of nitrogen-water co-limitation in Mediterranean agro-ecosystems. Aust. J. Agric. Res. 56: 1151-1157

Sage RF, Pearcy RW (1987) The nitrogen use efficiency of C3 and C4 plants. II. Leaf nitrogen effects on the gas exchange characteristics of Chenopodium album L.and maranthus retroflexus L. Plant Physiol. 84: 959-963

Sale PJ (1973) Production of vegetable crops in a region of high solar inputs. Aust. J. Agric. Res. 24:733-749.

Samuel G (2006) Intensification of Smallholder Agriculture in Ethiopia: Options and Scenarios. Paper presented in the Future Agricultures Consortium Meeting at the Institute of Development Studies. 20-22 March, Addis Ababa, Ethiopia

Schafer-Pregl R, Ritter E, Concilio L, Hesselbach J, Lovatti L, Walkemeier B, Thelen H, Salamini F, Gebhardt C (1998) Analysis of quantitative trait loci (QTLs) and quantitative trait alleles (QTAs)for potato tuber yield and starch content. Theor. Appl. Genet. 97: 834-846 Scheible WR, Morcuende R, Czechowski T, Fritz C, Osuna D, Palacios-Rojas N, Schindelasch D, Thimm O, Udvardi MK, Stitt M (2004) Genome-wide reprogramming of primary and secondary metabolism, protein synthesis, cellular growth processes, and the regulatory infrastructure of Arabidopsis in response to nitrogen. Plant Physiol. 136: 24832499

Schlede H (1989) Distribution of acid soils and liming materials in Ethiopia. Ethiopian Institute of Geological Survey. Addis Ababa: Ethiopian Ministry of Mines and Energy.

Sengupta K, Karatia AS (1971) Path co-efficient analysis for some characters in soybean. Indian J. Genet. 31: 290-95

Sharifi M, Zebarth BJ, Coleman W (2007) Screening for potato nitrogen use efficiency using a recirculating hydroponic system., in Zhu Z, Minasi K, and Xing G. (eds.) Proceedings of the 
3rd international nitrogen conference contributed papers, October 12-16, 2004, Nanjing, China, Science Press, Monmouth Junction, NJ, USA, pp 285-290

Simko I, and $\mathrm{Hu} \mathrm{J}$ (2008) Population structure in cultivated lettuce and its impact on association mapping. J. Amer. Soc. Hort. Sci. 133(1): 61-68

Simko I, Costanzo S, Haynes KG, Christ BJ (2004) Mapping genes for resistance to Verticillium albo-atrum in tetraploid and diploid potato populations using haplotype association test and genetic linkage analysis. Mol. Genet. Genomics 271: 522-531

Simko I, McMurry S, Yang HM, Manschot A, Davies PJ, Ewing EE (1997) Evidence from Polygene Mapping for a Causal Relationship between Potato Tuber Dormancy and Abscisic Acid Content. Plant Physiol. 115: 1453-1 459

Simmonds NW (1991) Selection for local adaptation in a plant breeding programme. Theor. Appl. Genet. 82: 363-367

Simmonds NW (1995) Potatoes. In: Smartt J, Simmonds NW (eds) Evolution of crop plants, 2nd edition.

Sinebo W, Gretzmacher R, and Edelbauer A (2002) Environment of selection for grain yield in low fertilizer input in barley. Field Crops Res. 74:151-162

Singh P, Narayana SS (1993) Biometrical techniques in plant breeding, first ed. Kalyani publishers, New Delhi, India

Singh RK, Chaudhary BD (1985) Biometrical methods in quantitative genetic analysis. Kalyani Publishers, New Delhi, India, Pp: 211-215

Slater JW (1968) The effect of night temperature on the initiation of the potato. Potato Res. $11: 14-32$

Smil V (1991) Population growth and nitrogen: an exploration of a critical existential link. Popul. Dev. Rev. 17: 569-601. doi:10.2307/1973598

Sozzani R, Busch W, Spalding EP, and Benfey PN (2014) Advanced imaging techniques for the study of plant growth and development. Trends Plant Sci. 19: 304-310. doi:10.1016/j.tplants.2013.12.003

Spooner DM (2009) DNA barcoding will frequently fail in complicated groups: an example in wild potatoes. Am. J. Bot. 96 (6): 1177-1189. doi: 10.3732/ajb.0800246

Spooner DM and Bamberg JB (1994) Potato genetic resources: sources of resistance and systematics. Am. Potato J. 71: 325-337 
Spooner DM, McLean K, Ramsay G, Waugh R, Bryan GJ (2005) A single domestication for potato based on multi-locus amplified fragment length polymorphism genotyping. Proc. Natl. Acad. Sci. USA. 102:14694-14699

Stark JC, Westermann DT, and Hopkins B G ( 2004) Nutrient management guidelines for Russet Burbank potatoes. Bull. 840. Univ. of Idaho, Moscow

Stich B, Maurer HP, Melchinger AE (2006) Comparison of linkage disequilibrium in elite European maize inbred lines using AFLP and SSR markers. Mol. Breed. 17(3): 217-226

Stich B, Mohring J, Piepho HP, Heckenberger M, Buckler ES, and Melchinger AE (2008) Comparison of mixed model approaches for association mapping. Genetics 178: 1745-1754 Thompson JA and Nelson RL (1998) Core set of primers to evaluate genetic diversity in soybean. Crop Sci. 38 (5):1356-1362

Thumma BR, Naidu BP, Chandra A, Cameron DF, Bahnisch LM, Liu C (2001) Identification of causal relationships among traits related to drought resistance in Stylosanthes Scabra using QTL analysis. J. Exp. Bot. 52: 203-214

Tiemens-Hulscher M, Lammerts van Bueren LT, Hutten RCB (2012) Potato: improving organic cultivars including a participatory approach. In: Lammerts van Bueren ET, Myers JR (eds) Organic crop breeding. Wiley-Blackwell, Hoboken, pp:227-233

Tilman D, Cassman KG, Matson PA, Naylor R, and Polasky S (2002) Agricultural sustainability and intensive production practices. Nature 418: 671-677

Tuberosa R, Collins N C, Tardieu F (2008) Quantitative trait loci and crop performance under abiotic stress: Where do we stand? Plant Physiol.147: 469-486

Tyler KB, Broadbent FE, Bishop JC (1983) Efficiency of nitrogen uptake by potatoes. Am. Potato J. 60:261-269

Van Dam JP, Kooman L, Struik PC (1996) Effect of temperature and photoperiod on early growth and final number of tubers in potato(Solanum tuberosum L). Potato Res. 39 (1) :51-62 Van den Berg J, Ewing E, Plaisted R, McMurry S, Bonierbale M (1996) QTL analysis of potato tuber. Theor. Appl. Genet. 93: 317-324

Van den Berg J, Ewing E, Plaisted R, McMurry S, Bonierbale M (1996) QTL analysis of potato tuber. Theor. Appl. Genet. 93: 317-324

Van Eck HJ, Jacobs JME, Stam P, Ton J, Stiekema WJ, Jacobsen E (1994) Multiple alleles for tuber shape in diploid potato detected by qualitative and quantitative genetic analysis using RFLPs. Genetics 137:303-309 
Van Ooijen JW (2006) Join Map 4.0, software for the calculation of genetic linkage maps in experimental populations of diploid species. Plant Research International, Wageningen, Netherlands

Van Ooijen JW (2009) Map QTL 6.0, software for the mapping of quantitative trait loci in experimental populations of diploid species. Kyazma B.V, Wageningen, Netherlands Visker MHPW, Heilersig HJB, Kodde LP, Van de Weg WE, Voorrips RE, Struik PC, Colon LT (2005) Genetic linkage of QTLs for late blight resistance and foliage maturity type in six related potato progenies . Euphytica 143: 189-199 Doi: 10.1007/s10681-005-3444-8 Volkov RA, Komarova NY, Panchuk II, and Hemleben V (2003) Molecular evolution of rDNA external transcribed spacer and phylogeny of sect. Petota (genus Solanum). Mol. Phylogenet. Evol. 29 (2): 187-202

Voorrips R, Gort G, and Vosman B (2011) Genotype calling in tetraploid species from biallelic marker data using mixture models. BMC Bioinformatics: 12:172

Vos J (1997) The nitrogen response of potato ( Solanum tuberosum L) in the field: nitrogen uptake and yield, harvest index and nitrogen concentration. Potato Res. 40: 237-248

Vos J, and Mackerron DKL (2000) Basic concepts of the management and supply of nitrogen and water in potato production. In: A.J., Haverkort and D.K.L. Mackerron (eds), management of nitrogen and water in potato production. Wageningen, the Netherlands, Pp:15-33

Vos J (1995) The effects of nitrogen supply and stem density on leaf attributes and stem branchingin potato (Solanum tuberosum L.). Potato Res. 38:271-279

Vos J (2009) Nitrogen responses and nitrogen management in potato. Potato Res. 52(4):305317

Vos J and Biemond H (1992) Effects of nitrogen on the development and growth of the potato plant.1. Leaf appearance, expansion growth, life spans of leaves and stem branching. Ann. Bot. 70:27-35

Vos P, Uitdewilligen JAML, Voorrips R, Visser R, van Eck H (2015) Development and analysis of 20K SNP array for potato (Solanum tuberosum): an insight into the breeding history. Theor. Appl. Genet.128(12): 2387-2401

Walcroft AS, Whitehead D, Silvester WB, Kelliher FM (1997) The response of photosynthetic model parameters to temperature and nitrogen concentration in Pinus radiata D. Don. Plant Cell Environ. 20:1338-1348 
Watanabe KN (2002) Challenges in biotechnology for abiotic stress tolerance on roots and tubers. JIRCAS Working Report: 75-83

Wei D, Cui K, Ye G, Pan J, Xiang J, Huang J, Nie L (2012) QTL mapping for nitrogen- use efficiency and nitrogen deficiency tolerance traits in rice. Plant Soil 359: 281-295, Doi: 10:1007/s11104-012-1142-6

Welcker C, Boussuge B, Benciveni C, Ribaut JM, Tardieu F (2007) Are source and sink strengths genetically linked in maize plants subjected to water deficit? A QTL study of the responses of leaf growth and anthesis- silking interval to water deficit. J. Exp. Bot. 58:339349

Went FW (1959) Effects of environments of parent and grandparent generations of tuber production of potatoes. Am. J. Bot. 46:277-282

Werij JS, Furrer H, van Eck HJ, Visser RGF, Bachem CWB (2012) A limited set of starch related genes explain several interrelated traits in potato. Euphytica 186: 501-516. Doi: 10.1007/s10681-012-0651-y

Werij JS, Kloosterman B, Celis-Gamboa C, de Vos CH, America T, Visser RG, Bachem CW (2007) Unravelling enzymatic discoloration in potato through a combined approach of candidate genes, QTL, and expression analysis. Theor. Appl. Genet. 115(2):245-252

Westcott B (1986) Some methods of analysis genotype-environment interaction. Heredity 56: 243-53

Westermann DT (2005) Nutritional requirements of potatoes. Am. J. Potato Res. 82:301-307

Williams CN, Uzo JO, and Peregrine WTH (1991) Vegetable production in the tropics. Longman Scientific and Technical, Essex: Intermediate Tropical Agriculture Series

Winkler E (1971) Potato cultivation in Tyrol. II. Photosynthetic efficiency and respiration in different potato varieties. Potato Res. 14:1-18

Witcombe JR, Hollington PA, Howarth CJ, Reader S, Steele KA (2008) Breeding for abiotic stresses for sustainable agriculture. Phil. Trans. R. Soc. B 363: 703-716

doi:10.1098/rstb.2007.2179

Woldegiorgis G (2013) Potato variety development strategies and methodologies in Ethiopia. In Seed potato tuber production and dissemination: experiences, challenges and prospects. Proceedings of the national workshop on seed potato tuber production and dissemination, 1214 March 2012, Bahir Dar, Ethiopia

Wolfe DW, Fereres E, and Voss RE (1983) Growth and yield Response of two Irish potato cultivars to various levels of applied water. Irrigation Sci. 3: 211-222 
Wu P, Luo A (1996) Investigation on genetic background of leaf chlorophyll content variation in rice under nitrogen stressed condition via molecular markers. Yi. Chuan. Xue. Bao. 23:431-38

Xu NY, Fok M, Zhang GW, Li J, Zhou ZG (2014) The application of GGE biplot analysis for evaluating test locations and mega-environment investigation of cotton regional trials. J. Integr. Agric. 13:1921-1933

$\mathrm{Xu}$ Y, Beachell H, McCouch SR (2004) A marker based approach to broadening the genetic base of rice in the USA. Crop Sci. 44: 1947-1959

Yamaguchi J, Tanaka A (1990) Quantitative observation on the root system of various crops growing in the field. Soil Sci. Plant Nutr. 36:483-493. Doi: 10.1080/00380768.1990.10416917

Yamaya T, Obara M, Nakajima H, Sasaki S (2002) Genetic manipulation and quantitativetrait loci mapping for nitrogen recycling in rice. J. Exp. Bot. 53:917-25

Yan W (2001) GGE biplot-a windows application for graphical analysis of multi-environment trial data and other types of two-way data. Agron. J. 93:1111-1118

Yan W (2002) Singular-value partitioning in biplot analysis of multi-environmental trial data. Agron. J. 94:990-996

Yan W (2014) Crop variety trials: Data management and analysis, Johan Wiley and Sons Inc. pp.133-162

Yan W and Hunt, LA (1998) Genotype x environment interaction and crop yield. Plant Breed Rev. 16: 135 - 178

Yan W, Hunt LA, Sheng Q, Szlavnics Z (2000) Cultivar evaluation and mega-environment investigation based on GGE biplots. Crop Sci. 40:597-605

Yan W, Kang MS (2003) GGE biplot analysis: a graphical tool for breeders, geneticists, and agronomists. CRC Press, New York

Yan W, Kang MS, Ma B, Woods S, Cornelius PL (2007) GGE biplot vs. AMMI analysis of genotype-by-environment data. Crop Sci. 47:643-655

Yan W, Pageau D, Frégeau-Reid J, Durand J (2011) Assessing the representativeness and repeatability of test locations for genotype evaluation. Crop Sci. 51: 1603-1610

Yigzaw D, Fentahun M, and Tesfaye A (2008) Performance Stability Analysis of Potato Varieties under Rainfed and Irrigated Potato Production Systems in Northwestern Ethiopia. Eth.J.Sci \& Technol. 5(2):90-98 
Yin X, Goudriaan J, Lantinga EA, Vos J, Spiertz HJ (2003) A flexible sigmoid function of determinate growth. Ann. Bot. 91 (3):361-371

Yuan F-M, Bland WL (2005) Comparison of light- and temperature-based index models for potato (Solanum tuberosum L.) growth and development. Am. J. Potato. Res. 82:345-352 doi:10.1007/BF02871965

Zaag DE, and Burton WG (1978) Potential yield of Irish potato crop and its limitations, survey paper, $7^{\text {th }}$ Triennial conference of the European Assoc. For Irish potato research, Warsaw, Poland, Pp.7-22

Zavala-Garcia F, Bramel-Cox PJ, Eastin JD, Witt MD, and Andrews DJ (1992) Increasing the efficiency of crop selection for unpredictable environments. Crop Sci. 32:51-57

Zebarth BJ, Tai G, Tarn R, de Jong H, and Milburn PH (2004a) Nitrogen use efficiency characteristics of commercial potato cultivars. Can. J. Plant Sci. 84:589-598

Zebarth BJ, Leclerc Y, and Moreau G (2004b) Rate and timing of nitrogen fertilization of russet burbank potato: Nitrogen use efficiency. Can. J. Plant Sci. 84: 845-854

Zebarth BJ, Leclerc Y, and Moreau G (2004b). Rate and timing of nitrogen fertilization of russet burbank potato: Nitrogen use efficiency. Can. J. Plant Sci.84: 845-854

Zebarth BJ, Tarn TR, Jong Hde, Murphy A (2008). Nitrogen use efficiency characteristics of andigena and diploid Potato selections. Am. J. Potato Res. 85: 210-218

Zemba AA, Wuyep SZ, Adebayo AA, Jahknwa CJ (2013) Growth and yield response of Irish potato to climate in Jos-South, plateau state, Nigeria. GJHSS 13 (5): 12-18

Zhang F, Cui Z, Wang J, Li C, Chen X (2007) Current status of soil and plant nutrient management in China and improvement strategies. Chinese Bull. Bot. 24(6): 687-94

Zheng Z-L (2009). Carbon and nitrogen nutrient balance signalling in plants. Plant Signal Behav. 4: 584-591. doi:10.4161/psb.4.7.8540

Zhu C, Gore M, Buckler ES, and Yu J (2008) Status and Prospects of Association Mapping in Plants. Plant. Genome 1: 1-20

Zobel RW, Wright MJ, Gauch HG (1988) Statistical analysis of a yield trial. Agron. J. 80:388-393

Zvomuya F, Rosen CJ, and Miller JC (2002). Response of Russet Norkotah clonal selection to nitrogen fertilization. Am. J. Potato Res. 79: 231-239. doi:10.1007/BF02986355 


\section{Supplementary data}

\section{Chapter 2}

Supplementary table 1. List of potato cultivars with their pedigrees involved in this Study

\begin{tabular}{|c|c|c|c|}
\hline Variety name & Year of release & country & Pedigree \\
\hline Adora & 1990 & NETH & Primura $\mathrm{x}$ Alcmaria \\
\hline Agria & 1985 & GER & Quarta x Semio \\
\hline Almera & 1999 & NETH & BM 77-2102 x AR 80-31-20 \\
\hline Ambition & 2007 & NETH & Adora x Quinta \\
\hline Arinda & 1993 & NETH & Vulkano x AR 74-78-1 \\
\hline Asterix & 1991 & NETH & Cardinal x VE- 70-9 \\
\hline Bafana & 2009 & NETH & Victoria $\mathrm{x}$ Felsina \\
\hline Agata & 1990 & NETH & BM 52-72 x Sirco \\
\hline Agerie & & ETHIO & Local cultivar \\
\hline Annabelle & 2001 & NETH & Nicola x Monalisa \\
\hline Arizona & 2009 & NETH & UK 150-19 D 22 x mascott \\
\hline Ater-Abeba & & ETHIO & Local cultivar \\
\hline Awash & 1991 & ETHIO & $\mathrm{I}-1058 \mathrm{~B} \times 700111$ \\
\hline Baraka & 1971 & NETH & SVP 50-358 x Avenir \\
\hline Bartina & 1988 & NETH & Saturna x ZPC 62-75 \\
\hline Belete & 2009 & ETHIO & 397170.16 x 389746.2 \\
\hline Bellini & 2001 & NETH & Mondial x Felsina \\
\hline Berber & 1984 & NETH & Alcmaria x Ropta P 365 \\
\hline Bintje & 1910 & NETH & Munstersen x Jaune d' or(Fransen) \\
\hline Caesar & 1990 & NETH & Monalisa x Ropta B1178 \\
\hline Canberra & 2007 & NETH & Latona $\mathrm{x}$ RedScarlett \\
\hline Carlita & 1991 & NETH & Jaerla x Provita \\
\hline Carrera & 1999 & NETH & Allard x Concurrent \\
\hline Challenger & 2008 & NETH & Aziza $\mathrm{x}$ Victoria \\
\hline Charlotte & 1981 & FRA & Hansa x Danae \\
\hline Cleopatra & 1980 & NETH & ZPC 50-35 x Desiree \\
\hline Colomba & 2011 & NETH & Carrera x Agata \\
\hline Compass & 2011 & NETH & Pallas x Voyager \\
\hline Courage & 1998 & NETH & Lady Rosetta x HZ 81 H 202 \\
\hline Crisps4all & 2008 & NETH & RZ 85-238 x RZ 87-44 \\
\hline Dagim & 2013 & ETHIO & Not available \\
\hline Desiree & 1962 & NETH & Urgenta $x$ Deesche \\
\hline Evora & 2011 & NETH & LEE 92 - $196 \mathrm{x}$ Valor \\
\hline Fabula & 1997 & NETH & Monalisa x Hudson \\
\hline Faluka & 2006 & NETH & Armundo x Arielle \\
\hline Felsina & 1992 & NETH & Morene x Gloria \\
\hline Flamenco & 2013 & NETH & Red Scarlett x Red Cloud \\
\hline
\end{tabular}




\begin{tabular}{|c|c|c|c|}
\hline Variety name & Year of release & country & Pedigree \\
\hline Frisia & 1988 & NETH & ZPC 69 C $160 \times$ AM 66-42 \\
\hline Guassa & 2002 & ETHIO & Not available \\
\hline Gudenie & 2006 & ETHIO & Not available \\
\hline Hansa & 1957 & GER & Oberarnbacher Fruuhe X Flava \\
\hline Hermes & 1973 & AUT & DDR 5158 x Sw 163/55 \\
\hline Innovator & 1999 & NETH & Shepody x RZ 84-2580 \\
\hline Ivory Russet & 2011 & NETH & RZ 93 - 7105 x Innovator \\
\hline Jaerla & 1969 & NETH & Sirtema x MPI 19268 \\
\hline Jazzy & 2010 & NETH & Franceline x Cupido \\
\hline Kastelli & 2011 & NETH & Mondial x Felsina \\
\hline Kennebec & 1948 & USA & USDA B 127 x USDA 96-56 \\
\hline Kondor & 1984 & NETH & KONST 61-333 x WILIA \\
\hline Kuras & 1996 & NETH & BRDA (= PG 285) x VK 69-491 \\
\hline Kuroda & 1998 & NETH & AR 76-199-3 x KONST 80-1407 \\
\hline Lady Christl & 1996 & NETH & WS 73- 3-391 x Mansour \\
\hline Lady Claire & 1996 & NETH & Agria x KW 78-34-470 \\
\hline Lady Rosetta & 1988 & NETH & Cardinal x VTN 62-33-3 \\
\hline Leonardo & 1994 & NETH & Edzina x Ropta D 540 \\
\hline Liseta & 1988 & NETH & Spunta x VE 66-295 \\
\hline Lucinda & 2011 & NETH & Vivaldi x Carrera \\
\hline Marabel & 1993 & NETH & Nena x MA 75-364 \\
\hline Marfona & 1977 & NETH & Primura x Konst 51-123 \\
\hline Marilyn & 2006 & NETH & Nicola x Pomfine \\
\hline Maris Piper & 1963 & GB & Y 22/6 (Arran cairn x Herald \\
\hline Markies & 1997 & NETH & Fianna x Agria \\
\hline Melody & 2001 & NETH & VE $74-45$ x W 72-22 - 496 \\
\hline Memphis & 2012 & NETH & MUH 92-13 x MUH 91-13 \\
\hline Monalisa & 1982 & NETH & Bierma A1-287 x colmo \\
\hline Mondial & 1987 & NETH & Spunta x VE 66-295 \\
\hline Mozart & 2003 & NETH & Red Star x Caesar \\
\hline Navigator & 2013 & NETH & BRU 93 -136 x Victoria \\
\hline Nicola & 1973 & GER & Cluvia x 6430/101 \\
\hline Orchestra & 2007 & NETH & Maradona x Cupido \\
\hline Panther & 2011 & NETH & Innovator $\mathrm{x}$ Beets 84-85-32 \\
\hline Picasso & 1994 & NETH & Cara x Ausonia \\
\hline Premiere & 1979 & NETH & Civa x Provita \\
\hline Ramos & 2000 & NETH & Agria x VK 69-491 \\
\hline Red Scarlett & 1999 & NETH & ZPC 80-239 x Impalia \\
\hline Rodeo & 1999 & NETH & Mondial x Bimonda \\
\hline Ronaldo & 2011 & NETH & Red pontiac x RZ-84-67 \\
\hline Russet Burbank & 1908 & USA & Mutant of burbank \\
\hline Sagitta & 2006 & NETH & Gallia x RZ-86-2918 \\
\hline
\end{tabular}




\begin{tabular}{|c|c|c|c|}
\hline Variety name & Year of release & country & Pedigree \\
\hline Santana & 1994 & NETH & Spunta x Vk 69-491 \\
\hline Sante & 1983 & NETH & Y 66-13-636 x AM 66-42 \\
\hline Saturna & 1964 & NETH & Maritta x ( Re cord x CPC 1673(adg)) \\
\hline Shepody & 1980 & CAN & Bake king x F58050 \\
\hline Sifra & 2008 & NETH & Mondial x Robinta \\
\hline Sisay & 1991 & ETHIO & I-1058B x 700111 \\
\hline Spunta & 1968 & NETH & Bea x USDA 96-56 \\
\hline Sylvana & 2008 & NETH & Fabula $\mathrm{x}$ Xantia \\
\hline Taurus & 2008 & NETH & Panda x RZ 87-44 \\
\hline Timate & 1984 & NETH & Elvira x AM 66-42 \\
\hline Triplo & 2000 & NETH & Agria $x$ fresco \\
\hline Victoria & 1997 & NETH & Agria x Ropta J 861 \\
\hline Vivaldi & 1998 & NETH & TS 77-148 x Monalisa \\
\hline Volumia & 2004 & NETH & Mondial x Adora \\
\hline Voyager & 2003 & NETH & RZ 85-238 x Oblelix \\
\hline Vr 808 & 2009 & NETH & Lady Claire x Atlantic \\
\hline Zengena & 2001 & ETHIO & Not available \\
\hline Zina Red & 2013 & NETH & Symfonia x Amorosa \\
\hline
\end{tabular}

Origin: AUT $=$ Austria, CAN $=$ Canada, ETHIO, Ethiopia FRA $=$ France, GB $=$ Great Brtain, GER = Germnay, NETH = Netherlands, USA = United States of America

Supplementary Table 2. Intra-cluster (diagonal) and inter-cluster distance $\mathrm{D}^{2}$ among the 9 clusters at low $\mathrm{N}$

\begin{tabular}{|c|c|c|c|c|c|c|c|c|c|}
\hline Cluster & I & II & III & IV & $\mathbf{V}$ & VI & VII & VIII & IX \\
\hline I & 0.3 & 16.5 & $28^{* * *}$ & $42.1 * *$ & $35.1 * *$ & $125.5^{* * *}$ & $46.0^{* *}$ & $55.8^{* *}$ & $38.2 * *$ \\
\hline II & & 7 & $40.6^{* *}$ & $91.0 * *$ & $85.9 * *$ & $208.7^{* * *}$ & $49.5^{* *}$ & $51.4 * *$ & $62.8 * *$ \\
\hline III & & & 7.8 & $44.1 * *$ & $79.0 * *$ & $181.7 * *$ & $52.3 * *$ & $53.1 * *$ & $80.7 * *$ \\
\hline IV & & & & 7.8 & $27.6^{* *}$ & $65.4^{* *}$ & $88.1^{* *}$ & $115.9^{* *}$ & $80.3^{* *}$ \\
\hline $\mathbf{V}$ & & & & & 7.8 & $44.9^{* *}$ & $92.2 * *$ & $139.2^{* * *}$ & $52.6 * *$ \\
\hline VI & & & & & & 0.0 & $179.4^{* *}$ & $296.1 * *$ & $155.0^{* * *}$ \\
\hline VII & & & & & & & 0.0 & $139.0^{* * *}$ & $108.8^{* *}$ \\
\hline VIII & & & & & & & & 0.0 & $85.6^{* * *}$ \\
\hline IX & & & & & & & & & 0.0 \\
\hline
\end{tabular}

where $\chi^{2} 11=19.70$ significant at $0.05(*) ; \chi^{2} 11=24.73$ highly significant at $0.01(* *)$ 
Supplementary Table 3. Intra-cluster (diagonal ) and inter-cluster distance $\mathrm{D}^{2}$ among the 11 clusters at high $\mathrm{N}$

\begin{tabular}{|c|c|c|c|c|c|c|c|c|c|c|c|}
\hline Cluster & I & II & III & IV & V & VI & VII & VIII & IX & $\mathrm{X}$ & XI \\
\hline I & 0.86 & & & & & & & & & & \\
\hline II & $21.6^{*}$ & 5.9 & & & & & & & & & \\
\hline III & 12.3 & $27.8 * *$ & 4.5 & & & & & & & & \\
\hline IV & $40.7 * *$ & $34.8 * *$ & $23.6^{*}$ & 7.0 & & & & & & & \\
\hline V & 17.9 & $35.4 * *$ & $49.4^{* *}$ & $85.9^{* *}$ & 5.3 & & & & & & \\
\hline VI & $63.9 * *$ & $47.6 * *$ & $54.1 * *$ & $43.9 * *$ & $97.0 * *$ & 7.0 & & & & & \\
\hline VII & $113.8^{* * *}$ & $103.9^{* *}$ & $80.4^{* *}$ & $60.4 * *$ & $190.3^{* *}$ & $54.4 * *$ & 7.8 & & & & \\
\hline VIII & $29.2 * *$ & $77.5^{* *}$ & $51.4^{* *}$ & $94.3 * *$ & $38.0 * *$ & $148.5^{* *}$ & $205.1 * *$ & 0.0 & & & \\
\hline IX & $60.7 * *$ & $48.8 * *$ & $52.4 * *$ & $65.0 * *$ & $109.6^{* *}$ & $112.5^{* *}$ & $149.2^{* *}$ & $87.4 * *$ & 0.0 & & \\
\hline X & $27.0 * *$ & $41.9 * *$ & $33.8^{* *}$ & $76.1^{* *}$ & $47.3^{* *}$ & $84.4 * *$ & $107.9^{* *}$ & $79.5 * *$ & $104.1^{* *}$ & 0.0 & \\
\hline XI & $390.6^{* *}$ & $346.6^{* * *}$ & $345.3^{* *}$ & $279.2 * *$ & $520.1 * *$ & $298.6^{* *}$ & $308.1^{* *}$ & $483.1^{* *}$ & $223.4^{* *}$ & $457.7^{* *}$ & 0.0 \\
\hline
\end{tabular}


Supplementary Table 4. Cluster means for 97 potato cultivars studied for 12 quantitative traits at low $\mathrm{N}$

\begin{tabular}{|c|c|c|c|c|c|c|c|c|c|c|}
\hline \multirow[t]{2}{*}{ Traits } & \multicolumn{10}{|l|}{ Clusters } \\
\hline & I & II & III & IV & V & VI & VII & VIII & IX & $\begin{array}{l}\text { trait } \\
\text { means }\end{array}$ \\
\hline ATW & 50.6 & 42.6 & 28.9 & 28.4 & 55.8 & 43.8 & 25.4 & 47.4 & 77.6 & 44.5 \\
\hline TDM $\%$ & 12.9 & 12.7 & 12.0 & 16.0 & 15.9 & 17.7 & 17.9 & 9.1 & 17.6 & 14.6 \\
\hline DTM & 73.4 & 65.0 & 72.0 & 84.5 & 89.3 & 93.0 & 68.0 & 74.0 & 89.3 & 78.7 \\
\hline TNPP & 5.6 & 4.2 & 9.9 & 10.4 & 6.5 & 8.3 & 8.3 & 7.3 & 4.3 & 7.2 \\
\hline NUE & 39.9 & 27.3 & 39.2 & 56.1 & 65.0 & 76.8 & 45.1 & 30.7 & 61.4 & 49.1 \\
\hline TYPP & 270.7 & 178.8 & 276.9 & 297.5 & 350.1 & 384.0 & 212.5 & 309.5 & 295.8 & 286.2 \\
\hline UCC & 38.7 & 40.3 & 40.3 & 36.1 & 34.6 & 29.9 & 31.4 & 47.4 & 40.2 & 37.6 \\
\hline $\mathrm{t} 1$ & 17.7 & 16.2 & 16.9 & 19.1 & 20.7 & 21.3 & 16.1 & 18.1 & 15.4 & 17.9 \\
\hline $\mathrm{Te}$ & 35.6 & 31.2 & 35.3 & 39.8 & 40.8 & 44.4 & 31.6 & 33.7 & 38.7 & 36.8 \\
\hline AUC & 1008.3 & 651.0 & 1003.0 & 1295.0 & 1368.3 & 1846.9 & 1093.1 & 607.7 & 796.1 & 1074.4 \\
\hline $\mathrm{PH}$ & 29.7 & 22.4 & 27.6 & 38.0 & 42.4 & 41.0 & 24.7 & 27.1 & 33.8 & 31.8 \\
\hline SNPP & 3.6 & 3.4 & 6.1 & 3.7 & 3.6 & 2.3 & 6.4 & 3.5 & 3.1 & 4.0 \\
\hline
\end{tabular}

ATW $=$ Average tuber weight in $\mathrm{g}, \mathrm{TDM} \%=$ Tuber dry matter in percent, DTM $=$ Days to maturity, TNPP $=$ Tuber number plant $^{-1}$, NUE $=$ Nitrogen use efficiency $\left(\mathrm{kg} \mathrm{kg}^{-1}\right.$, TYPP $=$ Tuber yield plant ${ }^{-1}$ in $\mathrm{g}, \mathrm{UCC}=$ upper leaf chlorophyll content(SPAD readings), $\mathrm{t} 1=$ time point at which the canopy stabilized in thermal day $(\mathrm{td}), \mathrm{te}=$ complete canopy senesced in td, $\mathrm{AUC}=$ Area under the canopy curve $\%$ t.d, $\mathrm{PH}=$ Plant height in $\mathrm{cm}, \mathrm{SNPP}=$ Stem number plant $^{-1}$ 
Supplementary Table 5. Cluster means for 97 potato cultivars studied for 12 quantitative traits at high $\mathrm{N}$

\begin{tabular}{|c|c|c|c|c|c|c|c|c|c|c|c|c|}
\hline & clusters & & & & & & & & & & & $\begin{array}{l}\text { trait } \\
\text { means }\end{array}$ \\
\hline traits & I & II & III & IV & V & VI & VII & VIII & IX & $\mathrm{X}$ & XI & \\
\hline ATW & 61.2 & 41.0 & 63.5 & 49.0 & 53.6 & 65.9 & 70.1 & 39.5 & 26.8 & 94.1 & 30.0 & 54.1 \\
\hline TDM & 9.9 & 16.2 & 9.8 & 14.8 & 11.7 & 16.6 & 13.6 & 8.4 & 14.3 & 7.2 & 15.7 & 12.5 \\
\hline DTM & 71.0 & 70.9 & 75.3 & 79.6 & 66.9 & 88.9 & 87.6 & 65.0 & 67.3 & 79.3 & 90.0 & 76.5 \\
\hline TNPP & 7.4 & 9.6 & 8.6 & 9.5 & 5.7 & 8.9 & 11.5 & 8.0 & 12.8 & 6.8 & 21.0 & 10.0 \\
\hline NUE & 16.3 & 24.3 & 20.0 & 26.2 & 13.9 & 36.9 & 41.4 & 10.2 & 20.0 & 18.3 & 38.6 & 24.2 \\
\hline TYPP & 430.1 & 380.1 & 522.3 & 451.9 & 293.4 & 572.1 & 822.1 & 317.5 & 342.5 & 642.5 & 620.0 & 490.4 \\
\hline UCC & 43.6 & 43.9 & 40.4 & 41.5 & 45.8 & 43.4 & 44.4 & 45.5 & 40.6 & 49.1 & 42.0 & 43.7 \\
\hline $\mathrm{t} 1$ & 17.1 & 16.9 & 18.6 & 18.2 & 17.5 & 18.5 & 17.5 & 17.5 & 16.4 & 17.4 & 18.1 & 17.6 \\
\hline te & 33.8 & 34.3 & 36.0 & 36.7 & 33.2 & 39.7 & 39.1 & 30.6 & 32.4 & 37.8 & 39.1 & 35.7 \\
\hline AUC & 1337.3 & 1445.1 & 1645.3 & 1823.9 & 1039.0 & 1786.5 & 2407.8 & 1077.2 & 1558.9 & 1727.3 & 2217.4 & 1642.3 \\
\hline $\mathrm{PH}$ & 34.5 & 35.2 & 38.2 & 49.5 & 29.6 & 42.4 & 54.2 & 28.2 & 28.2 & 37.3 & 50.4 & 38.9 \\
\hline SNPP & 3.7 & 3.5 & 4.8 & 4.3 & 3.1 & 2.8 & 3.8 & 6.5 & 6.1 & 3.0 & 4.9 & 3.8 \\
\hline
\end{tabular}

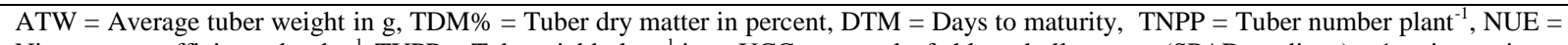
Nitrogen use efficiency kg kg${ }^{-1}$ TYPP $=$ Tuber yield plant ${ }^{-1}$ in $\mathrm{g}, \mathrm{UCC}=$ upper leaf chlorophyll content $(\mathrm{SPAD}$ readings), $\mathrm{t} 1=\mathrm{time}$ point at which the canopy stabilized in thermal day (td), te = complete canopy senesced in td, AUC = Area under the canopy curve $\%$ t.d, PH= Plant height in $\mathrm{cm}, \mathrm{SNPP}=$ Stem number plant $^{-1}$ 
Supplementary Table 6. Estimates of variance components, $\left.\mathrm{H}^{2}, \mathrm{PCV}\right)$ and GCV (GA\%) of measured traits at two contrasting $\mathrm{N}$ levels in Debre-Tabor

\begin{tabular}{|c|c|c|c|c|c|c|c|c|c|c|}
\hline Trait & Treat & Mean .range & G. mean & vp & $\mathrm{Vg}$ & ve & $\mathrm{H}^{2}$ & PCV & GCV & GA $\%$ \\
\hline \multirow[t]{2}{*}{$\mathrm{PH}$} & $\mathrm{LN}$ & $27.8-51.7$ & $36.0 \pm 5.5$ & 21.0 & 11.5 & 9.4 & 0.6 & 0.13 & 0.09 & 17.5 \\
\hline & $\mathrm{HN}$ & $28.7-66.7$ & $44.2 \pm 6.8$ & 31.0 & 20.4 & 10.6 & 0.7 & 0.13 & 0.10 & 21.0 \\
\hline \multirow[t]{2}{*}{ Vmax } & $\mathrm{LN}$ & $28.9-100.2$ & $53.3 \pm 12.5$ & 107.5 & 55.8 & 51.7 & 0.5 & 0.19 & 0.14 & 25.0 \\
\hline & $\mathrm{HN}$ & $39.9-101$ & $69.5 \pm 14.2$ & 150.6 & 102.3 & 48.3 & 0.7 & 0.18 & 0.15 & 28.7 \\
\hline \multirow[t]{2}{*}{ AUC } & $\mathrm{LN}$ & $602-2219$ & $1158 \pm 300.81$ & 62943.0 & 34954.0 & 27989.0 & 0.6 & 0.22 & 0.16 & 29.7 \\
\hline & $\mathrm{HN}$ & $758-2551$ & $1467 \pm 380.73$ & 103514.5 & 69226.0 & 34288.5 & 0.7 & 0.22 & 0.18 & 35.8 \\
\hline \multirow[t]{2}{*}{ UCC } & $\mathrm{LN}$ & $28.80-47.9$ & $39.3 \pm 3.0$ & 7.5 & 6.2 & 1.3 & 0.8 & 0.07 & 0.06 & 13.0 \\
\hline & $\mathrm{HN}$ & $35.1-59.8$ & $43.5 \pm 4.4$ & 18.2 & 17.4 & 0.8 & 0.9 & 0.10 & 0.10 & 19.8 \\
\hline \multirow[t]{2}{*}{ DTM } & $\mathrm{LN}$ & $48.0-81.5$ & $67.9 \pm 8.0$ & 43.7 & 23.3 & 20.5 & 0.5 & 0.10 & 0.07 & 13.0 \\
\hline & $\mathrm{HN}$ & $52.0-84.0$ & $68.6 \pm 7.1$ & 37.6 & 25.2 & 12.4 & 0.7 & 0.09 & 0.07 & 14.3 \\
\hline \multirow[t]{2}{*}{ TNPP } & $\mathrm{LN}$ & $3.5-13$ & $6.9 \pm 2.2$ & 4.1 & 3.4 & 0.7 & 0.8 & 0.30 & 0.27 & 54.6 \\
\hline & $\mathrm{HN}$ & $3.5-23$ & $8.2 \pm 3.1$ & 8.3 & 6.8 & 1.5 & 0.8 & 0.35 & 0.32 & 64.8 \\
\hline \multirow[t]{2}{*}{ TYPP } & $\mathrm{LN}$ & $180.0-622.9$ & $322.1 \pm 85.3$ & 5619.5 & 4106.0 & 1513.5 & 0.7 & 0.23 & 0.20 & 39.9 \\
\hline & $\mathrm{HN}$ & $202.5-1103.3$ & $460.52 \pm 173.99$ & 22711.5 & 15478.0 & 7233.5 & 0.7 & 0.33 & 0.27 & 53.0 \\
\hline \multirow[t]{2}{*}{ ATW } & $\mathrm{LN}$ & $24.75-93.13$ & $49.7 \pm 15.0$ & 170.4 & 122.4 & 48.1 & 0.7 & 0.26 & 0.22 & 44.7 \\
\hline & $\mathrm{HN}$ & $26.4-95.1$ & $59.6 \pm 18.7$ & 274.7 & 211.8 & 62.9 & 0.8 & 0.28 & 0.24 & 49.9 \\
\hline \multirow[t]{2}{*}{$\operatorname{TDM}(\%)$} & $\mathrm{LN}$ & $5.2-18.9$ & $11.5 \pm 3.8$ & 10.9 & 7.1 & 3.8 & 0.7 & 0.29 & 0.23 & 44.6 \\
\hline & $\mathrm{HN}$ & $4.6-21.3$ & $10.0 \pm 3.61$ & 9.9 & 6.7 & 3.2 & 0.7 & 0.31 & 0.26 & 50.6 \\
\hline \multirow[t]{2}{*}{ NUE } & $\mathrm{LN}$ & $17.0-119.2$ & $41.0 \pm 18.2$ & 274.9 & 220.3 & 54.6 & 0.8 & 0.40 & 0.36 & 73.3 \\
\hline & $\mathrm{HN}$ & $7.4-63.3$ & $17.2 \pm 10.9$ & 93.4 & 67.4 & 26.0 & 0.7 & 0.56 & 0.48 & 94.3 \\
\hline
\end{tabular}

$\mathrm{LN}=$ low nitrogen $\left(40 \mathrm{~kg} \mathrm{ha}^{-1}, \mathrm{HN}=\right.$ high nitrogen $\left(120 \mathrm{~kg} \mathrm{ha}^{-1}\right), \quad \mathrm{PH}=$ Plant height in $\mathrm{cm}, \mathrm{SNPP}=$ stem number plant ${ }^{-1,} \mathrm{~V}_{\max }=$ Maximum canopy cover in \%, AUC= Area under the canopy curve in \% thermal day $(\%$ td), UCC= Upper leaf chlorophyll content (SPAD readings), $\mathrm{DTM}=$ days to maturity, TNPP $=$ tuber number plant ${ }^{-1}, \mathrm{TYPP}=$ tuber yield $\mathrm{plant}^{-1}$ in $\mathrm{g}, \mathrm{ATW}=$ average tuber weight,in $\mathrm{g}, \mathrm{TDM}(\%)=$ tuber dry matter perceentage, NUE=nitrogen use efficiency $\left(\mathrm{kg} \mathrm{kg}^{-1}\right)$, G. mean = grand mean, $\mathrm{vp}=$ phenotypic variation, $\mathrm{vg}=$ genotypic variation, $\mathrm{ve}=$ environmental variation, $\mathrm{VgH}^{2}=$ broad sense heritability, $\mathrm{PCV}=$ phenotypic coefficient of variation, $\mathrm{GCV}=$ genotypic coefficient of variation, $\mathrm{GA} \%$ = genetic advance as percent of mean 
Supplementary table 7. Estimates of variance component, $\mathrm{H}^{2}, \mathrm{PCV}, \mathrm{GCV}$ and GA\% of measured traits at two contrasting $\mathrm{N}$ regimes in Injibara

\begin{tabular}{|c|c|c|c|c|c|c|c|c|c|c|}
\hline Trait & Treat & Mean range & G. mean & $\mathrm{Vp}$ & $\mathrm{Vg}$ & $\mathrm{Ve}$ & $\mathrm{H}^{2}$ & PCV & GCV & GA\% \\
\hline \multirow[t]{2}{*}{$\mathrm{PH}(\mathrm{cm})$} & LN & $9-39.5$ & $15.7 \pm 5.3$ & 25.3 & 22.8 & 2.5 & 0.9 & 0.32 & 0.30 & 62.4 \\
\hline & $\mathrm{HN}$ & $16.2-56.5$ & $27.3 \pm 8.1$ & 60.8 & 56.8 & 4.1 & 0.9 & 0.29 & 0.28 & 56.7 \\
\hline \multirow[t]{2}{*}{$\operatorname{Vmax}(\%)$} & $\mathrm{LN}$ & $23.5-53.6$ & $37.1 \pm 14.4$ & 28.7 & 9.6 & 19.1 & 0.3 & 0.14 & 0.08 & 26.3 \\
\hline & $\mathrm{HN}$ & $38.9-92.3$ & $62.0 \pm 9.4$ & 67.8 & 47.7 & 20.1 & 0.7 & 0.13 & 0.11 & 21.8 \\
\hline \multirow[t]{2}{*}{$\operatorname{AUC}(\% . t d)$} & LN & $457.4-1474.8$ & $866 \pm 348.7$ & 28778.0 & 16434.0 & 12344.0 & 0.6 & 0.20 & 0.15 & 47.4 \\
\hline & $\mathrm{HN}$ & $717-2481$ & $1366 \pm 317.4$ & 79930.0 & 58899 & 21031.0 & 0.7 & 0.21 & 0.18 & 35.4 \\
\hline \multirow[t]{2}{*}{ UCC } & $\mathrm{LN}$ & $32.3-46.9$ & $38.1 \pm 3.2$ & 8.4 & 6.7 & 1.7 & 0.8 & 0.08 & 0.07 & 14.0 \\
\hline & $\mathrm{HN}$ & $36.9-50.6$ & $43.3 \pm 3.6$ & 9.6 & 6.1 & 3.5 & 0.6 & 0.07 & 0.06 & 11.0 \\
\hline \multirow[t]{2}{*}{ DTM } & $\mathrm{LN}$ & $62.5-104.5$ & $80.0 \pm 8.4$ & 48.8 & 28.6 & 20.2 & 0.6 & 0.09 & 0.07 & 12.7 \\
\hline & $\mathrm{HN}$ & $59.0-107.0$ & $76.4 \pm 8.6$ & 60.5 & 47.7 & 12.8 & 0.8 & 0.10 & 0.09 & 18.2 \\
\hline \multirow[t]{2}{*}{ TNPP } & $\mathrm{LN}$ & $2.50-9.50$ & $4.7 \pm 1.3$ & 1.5 & 1.2 & 0.3 & 0.8 & 0.26 & 0.23 & 47.9 \\
\hline & $\mathrm{HN}$ & $4.0-19.0$ & $7.6 \pm 2.2$ & 4.0 & 3.2 & 0.8 & 0.8 & 0.26 & 0.23 & 47.4 \\
\hline \multirow[t]{2}{*}{ TYPP (g) } & $\mathrm{LN}$ & $135-320$ & $219.8 \pm 47.2$ & 1495.0 & 789 & 706.0 & 0.5 & 0.18 & 0.13 & 23.4 \\
\hline & $\mathrm{HN}$ & $235.0-700.0$ & $424.2 \pm 110.8$ & 7573.0 & 3970 & 3603.0 & 0.5 & 0.21 & 0.15 & 28.0 \\
\hline \multirow[t]{2}{*}{ ATW (g) } & $\mathrm{LN}$ & $20.9-83.8$ & $49.3 \pm 14.4$ & 167.7 & 127.9 & 39.8 & 0.8 & 0.26 & 0.23 & 45.9 \\
\hline & $\mathrm{HN}$ & $26.8-99.1$ & $58.8 \pm 18.4$ & 252.2 & 184.4 & 67.8 & 0.7 & 0.27 & 0.23 & 46.9 \\
\hline \multirow[t]{2}{*}{ TDM (\%) } & $\mathrm{LN}$ & $7.7-25.2$ & $14.9 \pm 4.1$ & 10.7 & 4.5 & 6.1 & 0.4 & 0.22 & 0.14 & 24.1 \\
\hline & $\mathrm{HN}$ & $6.8-18.8$ & $11.3 \pm 3.2$ & 8.2 & 6.4 & 1.8 & 0.8 & 0.25 & 0.22 & 44.9 \\
\hline \multirow[t]{2}{*}{ NUE } & $\mathrm{LN}$ & $20.2-69.6$ & $40.8 \pm 12.7$ & 95.3 & 38.2 & 57.1 & 0.4 & 0.24 & 0.15 & 25.6 \\
\hline & $\mathrm{HN}$ & $10.7-40.5$ & $20.0 \pm 6.5$ & 24.9 & 9.8 & 15.1 & 0.4 & 0.25 & 0.16 & 26.3 \\
\hline
\end{tabular}

$\mathrm{LN}=$ low nitrogen $\left(40 \mathrm{~kg} \mathrm{ha}^{-1}\right), \mathrm{HN}=$ high nitrogen $\left(120 \mathrm{~kg} \mathrm{ha}^{-1}\right), \quad \mathrm{PH}=$ Plant height in $\mathrm{cm}, \mathrm{SNPP}=$ stem number plant ${ }^{-1}, \mathrm{~V}_{\max }=\mathrm{Maximum}$ canopy cover in \%, AUC = Area under the canopy curve in \% thermal day $(\% \mathrm{td}), \mathrm{UCC}=$ Upper leaf chlorophyll content (SPAD readings), $\mathrm{DTM}=$ days to maturity, TNPP= tuber number plant ${ }^{-1}, \mathrm{TYPP}=$ tuber yield plant ${ }^{-1}, \mathrm{ATW}=$ average tuber weight in $\mathrm{g}, \mathrm{TDM}(\%)=$ tuber dry matter percentage, NUE=nitrogen use efficiency $\left(\mathrm{kg} \mathrm{kg}^{-1}\right)$, G. mean = grand mean, $\mathrm{VP}=$ phenotypic variation, $\mathrm{Vg}=$ genotypic variation, $\mathrm{Ve}$ = environmental variation, $\mathrm{VgH}^{2}=$ broad sense heritability, $\mathrm{PCV}=$ phenotypic coefficient of variation, $\mathrm{GCV}=$ genotypic coefficient of variation, $\mathrm{GA} \%$ = genetic advance as percent of mean 


\section{Chapter 3}

Supplementary Table 1. Analysis of variance for the influence of fertilizer $\mathrm{N}$ rate, cultivars, location nd their interaction over locations

\begin{tabular}{|c|c|c|c|c|c|c|c|c|c|c|c|}
\hline $\begin{array}{l}\text { Source of } \\
\text { Variations }\end{array}$ & $\mathrm{PH}$ & SNPP & $\mathrm{V}_{\max }$ & AUC & LCC & DTM & TDM & ATW & TNPP & TYPP & NUE \\
\hline N-lvels & ns & Ns & $\mathrm{ns}$ & $*$ & $\mathrm{~ns}$ & $\mathrm{~ns}$ & $\mathrm{~ns}$ & $\mathrm{~ns}$ & $*$ & ns & ns \\
\hline Genotypes & $* * *$ & $* * *$ & $* *$ & $* *$ & $* * *$ & $* * *$ & $* *$ & $* *$ & $* * *$ & $* * *$ & $* * *$ \\
\hline $\mathrm{NxG}$ & ns & Ns & $\mathrm{ns}$ & $\mathrm{ns}$ & $\mathrm{ns}$ & $\mathrm{ns}$ & $\mathrm{ns}$ & $* *$ & $* *$ & $\mathrm{~ns}$ & ns \\
\hline Location & $* * *$ & $* *$ & $* * *$ & $* * *$ & $* * *$ & $* * *$ & $\mathrm{~ns}$ & $* * *$ & $* * *$ & $* * *$ & $* * *$ \\
\hline $\mathrm{N} \times$ Loc & $* * *$ & $* * *$ & $* * *$ & $* * *$ & $* * *$ & $* * *$ & $* *$ & $* * *$ & $* * *$ & $* * *$ & $* * *$ \\
\hline G x Loc & $* * *$ & $\mathrm{Ns}$ & $* * *$ & $* * *$ & $* *$ & $* * *$ & ns & $* * *$ & $* * *$ & $* * *$ & $* * *$ \\
\hline N x G x Loc & ns & Ns & $*$ & $\mathrm{~ns}$ & $\mathrm{~ns}$ & $\mathrm{~ns}$ & ns & $\mathrm{ns}$ & $\mathrm{ns}$ & $\mathrm{ns}$ & ns \\
\hline
\end{tabular}

ns $=$ not significant, $*=$ significant at $\mathrm{P} \leq 0.05, * *=$ significant at $\mathrm{P} \leq 0.01$ and $* * *=$ significant at $\mathrm{P} \leq 0.001 \mathrm{PH}=\mathrm{Plant}$ Height, $\mathrm{SNPP}=$ stem number per plant, Vmax = maximum canopy cover, Total area under the canopy(AUC), LCC= Lower leaves chlorophyll content , DTM= days to maturity TDM $=$ Tuber Dry Matter\%, ATW= Average Tuber Weight, TNPP=Tuber Number Plant ${ }^{-1}$, TYPP $=$ Tuber Yield Plant $^{-1}$, NUE $=$ Nitrogen use efficiency 
Supplementary Table2. Mean performance of parents and progeny, estimates of genetic and non-genetic variance components, and heritability in different locations and $\mathrm{N}$-levels

\begin{tabular}{|c|c|c|c|c|c|c|c|c|c|c|}
\hline \multirow[t]{3}{*}{ Trait } & \multirow[t]{3}{*}{ Treat } & \multirow[t]{3}{*}{ Locations } & \multicolumn{5}{|c|}{ Performance } & \multicolumn{3}{|c|}{ variance } \\
\hline & & & \multicolumn{2}{|c|}{ parents } & \multicolumn{3}{|c|}{ Progeny } & \multirow[b]{2}{*}{$\sigma^{2} g$} & \multirow[b]{2}{*}{$\sigma^{2} \mathrm{e}$} & \multirow[b]{2}{*}{$\mathrm{H}^{2}$} \\
\hline & & & $\mathrm{C}$ & E & Min & Max & Mean & & & \\
\hline \multirow[t]{6}{*}{ DTE } & $\mathrm{LN}$ & Ko & 25 & 23 & 17 & 29 & 22 & 6.4 & 3 & 0.7 \\
\hline & & In & 30 & 28 & 20 & 40 & 27 & 10.7 & 6.2 & 0.6 \\
\hline & & DT & 25 & 24 & 17 & 50 & 26 & 51.09 & 6.9 & 0.9 \\
\hline & $\mathrm{HN}$ & Ko & 21 & 18 & 16 & 26 & 21 & 2.8 & 3.4 & 0.5 \\
\hline & & In & 28 & 24 & 20 & 36 & 25 & 10.4 & 5.2 & 0.7 \\
\hline & & DT & 25 & 24 & 17 & 49 & 25 & 43.2 & 5.3 & 0.9 \\
\hline \multirow[t]{6}{*}{ PH } & $\mathrm{LN}$ & Ko & 30 & 43 & 12 & 46 & 28 & 40.06 & 7.9 & 0.8 \\
\hline & & In & 26 & 27 & 11 & 40 & 24 & 36.8 & 7.5 & 0.8 \\
\hline & & DT & 35 & 38 & 23 & 46 & 32 & 12.5 & 17.2 & 0.4 \\
\hline & $\mathrm{HN}$ & Ko & 33 & 42 & 17 & 52 & 34 & 42.3 & 7.04 & 0.9 \\
\hline & & In & 36 & 37 & 16 & 57 & 33 & 51.2 & 12.5 & 0.8 \\
\hline & & DT & 42 & 51 & 24 & 56 & 39 & 28.3 & 11 & 0.7 \\
\hline \multirow[t]{6}{*}{ SNPP } & LN & Ko & 2 & 2 & 1 & 3 & 2 & 0.06 & 0.12 & 0.4 \\
\hline & & In & 1 & 2 & 1 & 3 & 2 & 0.06 & 0.12 & 0.4 \\
\hline & & DT & 1 & 1 & 1 & 4 & 2 & 0.11 & 0.2 & 0.4 \\
\hline & $\mathrm{HN}$ & Ко & 2 & 2 & 1 & 3 & 2 & 0.11 & 0.08 & 0.6 \\
\hline & & In & 2 & 2 & 1 & 3 & 2 & 0.06 & 0.12 & 0.3 \\
\hline & & DT & 2 & 3 & 1 & 3 & 2 & 0.07 & 0.2 & 0.3 \\
\hline \multirow[t]{6}{*}{ UCC } & LN & Кo & 45.9 & 47.2 & 34.4 & 58.2 & 45.5 & 12.2 & 7.2 & 0.6 \\
\hline & & In & 47.5 & 46.9 & 40.6 & 54.9 & 48 & 0.00 & 8.9 & 0.00 \\
\hline & & DT & 45.5 & 46.3 & 39.7 & 57.2 & 47.2 & 9.6 & 6.7 & 0.6 \\
\hline & $\mathrm{HN}$ & Ко & 46.6 & 48.5 & 37.2 & 63.6 & 48.7 & 5.7 & 18.8 & 0.2 \\
\hline & & In & 49.4 & 46 & 38.5 & 55.2 & 47.6 & 6.8 & 5 & 0.6 \\
\hline & & DT & 48.10 & 50.2 & 37.2 & 57.6 & 48.4 & 8.7 & 4.8 & 0.6 \\
\hline \multirow[t]{6}{*}{ LCC } & LN & Ko & 45.7 & 50 & 36.8 & 58.9 & 47.6 & 14.9 & 8.8 & 0.6 \\
\hline & & In & 47 & 44 & 37.30 & 56.4 & 46.7 & 0.00 & 13.2 & 0.00 \\
\hline & & DT & 45.5 & 45 & 38.2 & 57 & 46.7 & 9.3 & 8.4 & 0.5 \\
\hline & $\mathrm{HN}$ & Ko & 49.5 & 50.5 & 40.3 & 63.5 & 60 & 9.6 & 15.2 & 0.4 \\
\hline & & In & 47.2 & 45.3 & 38.7 & 55.7 & 47.4 & 5.04 & 7.5 & 0.4 \\
\hline & & DT & 50.5 & 50.10 & 38.4 & 58.7 & 48.6 & 10 & 8.4 & 0.5 \\
\hline
\end{tabular}




\begin{tabular}{|c|c|c|c|c|c|c|c|c|c|c|}
\hline \multirow[t]{3}{*}{ Trait } & \multirow[t]{3}{*}{ Treat } & \multirow[t]{3}{*}{ Locations } & \multicolumn{5}{|c|}{ Performance } & \multicolumn{3}{|c|}{ variance } \\
\hline & & & \multicolumn{2}{|c|}{ parents } & \multicolumn{3}{|c|}{ Progeny } & \multirow[b]{2}{*}{$\sigma^{2} g$} & \multirow[b]{2}{*}{$\sigma^{2} \mathrm{e}$} & \multirow[b]{2}{*}{$\mathrm{H}^{2}$} \\
\hline & & & $\mathrm{C}$ & E & Min & $\operatorname{Max}$ & Mean & & & \\
\hline \multirow[t]{6}{*}{$\operatorname{tm} 1$} & $\mathrm{LN}$ & Ko & 23.3 & 22.3 & 13.4 & 47.6 & 23.5 & 5.4 & 11.4 & 0.3 \\
\hline & & In & 6.3 & 7.8 & 2.7 & 13.8 & 9 & 2.7 & 3.7 & 0.3 \\
\hline & & DT & 16.2 & 16.6 & 7.5 & 39.5 & 15.4 & 4.2 & 7.3 & 0.4 \\
\hline & $\mathrm{HN}$ & Ко & 23.02 & 16.04 & 4.7 & 26.5 & 20 & 4.8 & 5.3 & 0.5 \\
\hline & & In & 9.5 & 10 & 4 & 13.4 & 10.2 & 1.2 & 1.8 & 0.4 \\
\hline & & DT & 16.9 & 17.01 & 8.5 & 19.9 & 16.2 & 3.6 & 0.9 & 0.8 \\
\hline \multirow[t]{6}{*}{$\mathrm{t} 1$} & $\mathrm{LN}$ & Ко & 32.7 & 33.7 & 20.2 & 44.6 & 35 & 2.9 & 15.03 & 0.2 \\
\hline & & In & 7.6 & 8.9 & 4.12 & 18.9 & 12.6 & 0.6 & 6.6 & 0.08 \\
\hline & & DT & 20.3 & 20.4 & 8.4 & 23.2 & 18.9 & 3.10 & 2.9 & 0.5 \\
\hline & $\mathrm{HN}$ & Ко & 37.04 & 20 & 22.10 & 42.9 & 33.8 & 9.6 & 14.10 & 0.4 \\
\hline & & In & 16 & 15.7 & 4.6 & 21 & 15.11 & 2.4 & 3.3 & 0.4 \\
\hline & & DT & 22.03 & 20.5 & 13.9 & 25.13 & 20.4 & 2.2 & 2.11 & 0.5 \\
\hline \multirow[t]{6}{*}{$\mathrm{t} 2$} & $\mathrm{LN}$ & Ко & 41.2 & 44.6 & 30.6 & 71.5 & 44.8 & 4.9 & 29.3 & 0.14 \\
\hline & & In & 17.10 & 18.3 & 12.2 & 35.05 & 18.8 & 0.4 & 12.04 & 0.03 \\
\hline & & DT & 21.9 & 21.3 & 12.6 & 27.4 & 22 & 2.9 & 2.14 & 0.6 \\
\hline & $\mathrm{HN}$ & Ko & 43.9 & 413 & 27.5 & 53.4 & 41 & 6.6 & 15.5 & 0.3 \\
\hline & & In & 18.9 & 18.2 & 13.8 & 38.4 & 19.2 & 0.00 & 8.7 & 0.00 \\
\hline & & DT & 25 & 24.6 & 15.2 & 29.8 & 23.2 & 2.3 & 3.6 & 0.4 \\
\hline \multirow[t]{6}{*}{$\mathrm{Te}$} & $\mathrm{LN}$ & Ko & 59.01 & 52.9 & 48.01 & 80.3 & 56.14 & 0.00 & 20.6 & 0.00 \\
\hline & & In & 41.9 & 29.3 & 26.13 & 127.9 & 34.5 & 0.00 & 102.8 & 0.00 \\
\hline & & DT & 43.4 & 44.2 & 35 & 46.08 & 42.07 & 3.4 & 1.8 & 0.7 \\
\hline & $\mathrm{HN}$ & Ко & 56.4 & 57.08 & 46.4 & 64.9 & 56.05 & 5.2 & 6.6 & 0.4 \\
\hline & & In & 29.2 & 33.7 & 26.5 & 109.4 & 34 & 8.5 & 67.7 & 0.11 \\
\hline & & DT & 42.7 & 44.09 & 35 & 47.5 & 42.9 & 4.3 & 2 & 0.7 \\
\hline \multirow[t]{6}{*}{ Vmax } & $\mathrm{LN}$ & Ko & 61.6 & 31.04 & 5.04 & 84.2 & 25.3 & 112.14 & 26.9 & 0.8 \\
\hline & & In & 24.6 & 24.3 & 11.6 & 39.7 & 25.3 & 9 & 9.14 & 0.5 \\
\hline & & DT & 35.3 & 40 & 17.4 & 47.3 & 30.03 & 4.8 & 34.6 & 0.12 \\
\hline & $\mathrm{HN}$ & Ко & 41.5 & 36.13 & 7.7 & 77.4 & 32.6 & 84 & 50.2 & 0.6 \\
\hline & & In & 54.6 & 40.7 & 12.9 & 71.2 & 42.4 & 72.13 & 31.2 & 0.7 \\
\hline & & DT & 56.8 & 62.4 & 20.5 & 74.02 & 45.4 & 51.2 & 51.5 & 0.5 \\
\hline
\end{tabular}




\begin{tabular}{|c|c|c|c|c|c|c|c|c|c|c|}
\hline \multirow[t]{3}{*}{ Trait } & \multirow[t]{3}{*}{ Treat } & \multirow[t]{3}{*}{$\underline{\text { Sites }}$} & \multicolumn{5}{|c|}{ Performance } & \multicolumn{3}{|c|}{ variance } \\
\hline & & & \multicolumn{2}{|c|}{ parents } & \multicolumn{3}{|c|}{ Progeny } & \multirow[b]{2}{*}{$\sigma^{2} \mathrm{~g}$} & \multirow[b]{2}{*}{$\sigma^{2} \mathrm{e}$} & \multirow[b]{2}{*}{$\mathrm{H}^{2}$} \\
\hline & & & $\mathrm{C}$ & $\mathrm{E}$ & Min & Max & Mean & & & \\
\hline \multirow[t]{6}{*}{ AUC } & $\mathrm{LN}$ & Ko & 2003.7 & 941.10 & 230.8 & 2946.8 & 787.2 & 99060 & 42172.5 & 0.7 \\
\hline & & In & 651 & 447.6 & 287.9 & 820.5 & 508.4 & 5992.5 & 9304.5 & 0.4 \\
\hline & & DT & 760 & 863 & 356 & 1061 & 651.5 & 1667 & 18553 & 0.08 \\
\hline & $\mathrm{HN}$ & Ko & 1292 & 1241 & 227 & 2705 & 1069.3 & 103850 & 58463.5 & 0.5 \\
\hline & & In & 927 & 790.2 & 317.6 & 1405.3 & 795.05 & 20410 & 12644 & 0.6 \\
\hline & & DT & 1303 & 1387 & 415 & 1678 & 993.10 & 30506 & 29473 & 0.5 \\
\hline \multirow[t]{6}{*}{ DTM } & $\mathrm{LN}$ & Кo & 117 & 114 & 91 & 123 & 115 & 9.3 & 9.8 & 0.5 \\
\hline & & In & 66 & 67 & 55 & 77 & 64 & 11 & 10 & 0.5 \\
\hline & & DT & 81 & 79 & 53 & 92 & 78 & 37.4 & 13.6 & 0.7 \\
\hline & $\mathrm{HN}$ & Кo & 112 & 112 & 91 & 121 & 112 & 12.2 & 20.8 & 0.4 \\
\hline & & In & 64 & 66 & 54 & 79 & 64 & 10.2 & 10.3 & 0.5 \\
\hline & & DT & 80 & 78 & 58 & 93 & 77 & 32.2 & 11.6 & 0.7 \\
\hline \multirow[t]{6}{*}{ NTPP } & $\mathrm{LN}$ & Ko & 10 & 8 & 3 & 19 & 9 & 11.5 & 2.9 & 0.8 \\
\hline & & In & 8 & 5 & 3 & 16 & 7 & 2.5 & 1.6 & 0.6 \\
\hline & & DT & 7 & 4 & 2 & 18 & 6 & 4.4 & 1.5 & 0.7 \\
\hline & $\mathrm{HN}$ & Ko & 16 & 13 & 3 & 30 & 12 & 21.2 & 5.4 & 0.8 \\
\hline & & In & 10 & 6 & 3 & 22 & 10 & 8.3 & 3.5 & 0.7 \\
\hline & & DT & 13 & 8 & 3 & 22 & 9 & 6.9 & 3.4 & 0.7 \\
\hline \multirow[t]{6}{*}{ TYPP } & LN & Ко & 500 & 300 & 70 & 700 & 300 & 0.01 & 0.00 & 0.8 \\
\hline & & In & 0.13 & 0.12 & 0.04 & 0.3 & 0.12 & 0.00 & 0.00 & 0.3 \\
\hline & & DT & 0.3 & 0.10 & 0.04 & 0.4 & 0.14 & 0.00 & 0.00 & 0.4 \\
\hline & $\mathrm{HN}$ & Ko & 0.6 & 0.5 & 0.04 & 0.9 & 0.4 & 0.02 & 0.01 & 0.7 \\
\hline & & In & 0.3 & 0.2 & 0.05 & 0.4 & 0.2 & 0.00 & 0.00 & 0.7 \\
\hline & & DT & 0.4 & 03 & 0.05 & 0.7 & 0.3 & 0.01 & 0.00 & 0.6 \\
\hline \multirow[t]{6}{*}{ ATW } & $\mathrm{LN}$ & Ko & 51 & 37.6 & 10.6 & 56.6 & 30.5 & 58.2 & 34.2 & 0.6 \\
\hline & & In & 16.5 & 23.11 & 8.7 & 31.5 & 18.4 & 11 & 8.8 & 0.6 \\
\hline & & DT & 38.05 & 25.3 & 10.9 & 44.9 & 22.8 & 27.09 & 17.3 & 0.6 \\
\hline & $\mathrm{HN}$ & Ko & 41.2 & 39.3 & 11.4 & 77.9 & 36 & 64.9 & 62.10 & 0.5 \\
\hline & & In & 24.2 & 28.6 & 11.4 & 41.7 & 20.02 & 25.3 & 8.9 & 0.7 \\
\hline & & DT & 31.7 & 34.14 & 15.4 & 56.7 & 31.04 & 33.4 & 27.4 & 0.6 \\
\hline
\end{tabular}




\begin{tabular}{|c|c|c|c|c|c|c|c|c|c|c|}
\hline \multirow[t]{3}{*}{ Trait } & \multirow[t]{3}{*}{ Treat } & \multirow[t]{3}{*}{$\underline{\text { Sites }}$} & \multicolumn{5}{|c|}{ Performance } & \multicolumn{3}{|c|}{ variance } \\
\hline & & & \multicolumn{2}{|c|}{ parents } & \multicolumn{3}{|c|}{ Progeny } & \multirow[b]{2}{*}{$\sigma^{2} \mathrm{~g}$} & \multirow[b]{2}{*}{$\sigma^{2} \mathrm{e}$} & \multirow[b]{2}{*}{$\mathrm{H}^{2}$} \\
\hline & & & $\mathrm{C}$ & E & Min & Max & Mean & & & \\
\hline \multirow[t]{6}{*}{ SG } & $\mathrm{LN}$ & Ko & 1.06 & 1.08 & 1.03 & 1.10 & 1.07 & 0.00 & 0.00 & 0.2 \\
\hline & & In & 1.08 & 1.08 & 1.04 & 1.08 & 1.07 & 0.00 & 0.00 & 0.00 \\
\hline & & DT & 1.08 & 1.09 & 1.04 & 1.13 & 1.07 & 0.00 & 0.00 & 0.2 \\
\hline & $\mathrm{HN}$ & Ко & 1.07 & 1.07 & 1.02 & 1.10 & 1.07 & 0.00 & 0.00 & 0.2 \\
\hline & & In & 1.05 & 1.08 & 1.04 & 1.08 & 1.07 & 0.00 & 0.00 & 0.2 \\
\hline & & DT & 1.06 & 1.08 & 1.03 & 1.10 & 1.06 & 0.00 & 0.00 & 0.2 \\
\hline \multirow[t]{6}{*}{ TDM\% } & $\mathrm{LN}$ & Ко & 15.6 & 20.5 & 10.3 & 24.6 & 18.3 & 2.8 & 10.4 & 0.2 \\
\hline & & In & 20.03 & 20.5 & 12.07 & 21.3 & 19 & 0.00 & 3.7 & 0.00 \\
\hline & & DT & 20.91 & 22.6 & 11.5 & 31.01 & 19.3 & 1.6 & 7.5 & 0.2 \\
\hline & $\mathrm{HN}$ & Ko & 18.5 & 18 & 6.7 & 24.7 & 18.14 & 2.9 & 9.09 & 0.2 \\
\hline & & In & 14.5 & 21.2 & 11.7 & 21.6 & 18.4 & 0.9 & 4.9 & 0.2 \\
\hline & & DT & 15.7 & 20.4 & 9.3 & 24.6 & 17.04 & 2.7 & 9.01 & 0.2 \\
\hline \multirow[t]{6}{*}{ NUE } & $\mathrm{LN}$ & Кo & 78.9 & 59.2 & 12.2 & 105.2 & 44.4 & 178.8 & 187.7 & 0.5 \\
\hline & & In & 29.11 & 26.07 & 7.8 & 63.09 & 24.4 & 15.6 & 52.9 & 0.2 \\
\hline & & DT & 65.5 & 28.02 & 7.6 & 79.7 & 34.2 & 149.6 & 78.3 & 0.7 \\
\hline & $\mathrm{HN}$ & Ko & 39.3 & 30.75 & 3.5 & 55.3 & 24.6 & 73.08 & 34.8 & 0.7 \\
\hline & & In & 13.04 & 14.6 & 3.2 & 34.4 & 12.5 & 11.2 & 11.2 & 0.5 \\
\hline & & DT & 27.6 & 23.2 & 4.14 & 34.3 & 17.7 & 17.7 & 20.8 & 0.5 \\
\hline
\end{tabular}

$\mathrm{DTE}=$ days to emergence, $\mathrm{Ph}=\mathrm{Plant}$ Height in $\mathrm{cm}, \mathrm{SNPP}=$ Stem Number Plant ${ }^{-1}, \mathrm{UCC}=$ upper leaves chlorophyll content(SPAD readings), $\mathrm{LCC}=$ Lower leaves chlorophyll content $(\mathrm{SPAD}$ readings $), \mathrm{DTM}=$ days to maturity TNPP=Tuber Number Plant ${ }^{-1}, \mathrm{TYPP}=$ Tuber Yield Plant ${ }^{-}$ ${ }^{1}$ in $\mathrm{g}$, ATW $=$ Average Tuber Weight in $\mathrm{g}, \mathrm{SG}=$ Specific Gravity $\left(\mathrm{g} \mathrm{g}^{-1}, \mathrm{TDM} \%=\right.$ Tuber Dry Matter percentage, NUE $=$ Nitrogen use efficiency $\left(\mathrm{kg} \mathrm{kg}^{-1}\right), \mathrm{tm} 1=$ inflection point of canopy growth in thermal day $(\mathrm{td}), \mathrm{t} 1=$ time for maximum canopy in td, $\mathrm{t} 2=$ time on set of canopy declining in td, te $=$ time canopy cover zero in td, Vmax = maximum canopy cover in $\%$, AUC $=$ total area under the canopy $\%$ td , Locations : KO = Koga, In = Injibara, DT = Debre-Tabor, Min = minimum mean, Max = maximum mean, $\sigma^{2} \mathrm{~g}=$ genotypic variance, $\sigma^{2} \mathrm{e}=$ environmental variance, $\mathrm{H}^{2}=$ broad sense heritability $\mathrm{LN},=$ low $\mathrm{N}$ level $\left(40 \mathrm{~kg} \mathrm{ha}^{-1}, \mathrm{HN}=\right.$ high $\mathrm{N}$ level $\left(120 \mathrm{~kg} \mathrm{ha}^{-1}\right), \mathrm{Ko}=\mathrm{Koga}$, In= Injibara, DT= Debre-Tabor 


\section{Chapter 4}

Supplementary Table 1. List of potato cultivars used in the association Panel, their year of release, origin, pedigree and market niche

\begin{tabular}{|c|c|c|c|c|}
\hline Variety name & Year & Origin & Parentage & Market niche \\
\hline Kennebec & 1948 & USA & USDA B 127 x USDA 96-56 & ancient cultivar \\
\hline Agata & 1990 & NETH & BM 52-72 x Sirco & fresh consumption \\
\hline Almera & 1999 & NETH & BM 77-2102 x AR 80-31-20 & fresh consumption \\
\hline Ambition & 2007 & NETH & Adora x Quinta & fresh consumption \\
\hline Annabelle & 2001 & NETH & Nicola x Monalisa & fresh consumption \\
\hline Arinda & 1993 & NETH & Vulkano x AR 74-78-1 & fresh consumption \\
\hline Bafana & 2009 & NETH & Victoria $\times$ Felsina & fresh consumption \\
\hline Bartina & 1988 & NETH & Saturna x ZPC 62-75 & fresh consumption \\
\hline Bellini & 2001 & NETH & Mondial x Felsina & fresh consumption \\
\hline Berber & 1984 & NETH & Alcmaria x Ropta P 365 & fresh consumption \\
\hline Bintje & 1910 & NETH & Munstersen x Jaune d' or(Fransen) & fresh consumption \\
\hline Charlotte & 1981 & FRA & Hansa x Danae & fresh consumption \\
\hline Desiree & 1962 & NETH & Urgenta $x$ Deesche & fresh consumption \\
\hline Fabula & 1997 & NETH & Monalisa $\mathrm{x}$ Hudson & fresh consumption \\
\hline Hansa & 1957 & GER & Oberarnbacherfruuhe X Flava & fresh consumption \\
\hline Jaerla & 1969 & NETH & Sirtema x MPI 19268 & fresh consumption \\
\hline Kastelli & 2011 & NETH & Mondial x Felsina & fresh consumption \\
\hline Kondor & 1984 & NETH & KONST 61-333 x WILIA & fresh consumption \\
\hline Kuroda & 1998 & NETH & AR 76-199-3 x KONST 80-1407 & fresh consumption \\
\hline Lady Christl & 1996 & NETH & WS 73- 3-391 x Mansour & fresh consumption \\
\hline Liseta & 1988 & NETH & Spunta x VE 66-295 & fresh consumption \\
\hline Marabel & 1993 & NETH & Nena x MA 75-364 & fresh consumption \\
\hline Marfona & 1977 & NETH & Primura x Konst 51-123 & fresh consumption \\
\hline Marilyn & 2006 & NETH & Nicola x Pomfine & fresh consumption \\
\hline Markies & 1997 & NETH & Fianna x Agria & fresh consumption \\
\hline Monalisa & 1982 & NETH & Bierma A1-287 x colmo & fresh consumption \\
\hline Mondial & 1987 & NETH & Spunta x VE 66-295 & fresh consumption \\
\hline Nicola & 1973 & GER & Cluvia x 6430/101 & fresh consumption \\
\hline Orchestra & 2007 & NETH & Maradona x Cupido & fresh consumption \\
\hline Picasso & 1994 & NETH & Cara $\mathrm{x}$ Ausonia & fresh consumption \\
\hline Ramos & 2000 & NETH & Agria x VK 69-491 & fresh consumption \\
\hline Red Scarlett & 1999 & NETH & ZPC 80-239 x Impalia & fresh consumption \\
\hline Sante & 1983 & NETH & Y 66-13-636 x AM 66-42 & fresh consumption \\
\hline Spunta & 1968 & NETH & Bea x USDA 96-56 & fresh consumption \\
\hline Timate & 1984 & NETH & Elvira x AM 66-42 & fresh consumption \\
\hline Canberra & 2007 & NETH & Latona x RedScarlett & general purpose \\
\hline Mozart & 2003 & NETH & Red Star x Caesar & general pupose \\
\hline Frisia & 1988 & NETH & ZPC 69 C 160 x AM 66-42 & general purpose \\
\hline Lucinda & 2011 & NETH & Vivaldi x Carrera & general purpose \\
\hline Melody & 2001 & NETH & VE $74-45 \times$ W $72-22-496$ & general purpose \\
\hline
\end{tabular}




\begin{tabular}{|c|c|c|c|c|}
\hline Variety name & Year & Origin & Parentage & Market niche \\
\hline Panther & 2011 & NETH & Innovator x Beets 84-85-32 & general purpose \\
\hline Rodeo & 1999 & NETH & Mondial x Bimonda & general purpose \\
\hline Sagitta & 2006 & NETH & Gallia x RZ-86-2918 & general purpose \\
\hline Sifra & 2008 & NETH & Mondial x Robinta & general purpose \\
\hline Sylvana & 2008 & NETH & Fabula x Xantia & general purpose \\
\hline Victoria & 1997 & NETH & Agria x Ropta J 861 & general purpose \\
\hline Vivaldi & 1998 & NETH & TS 77-148 x Monalisa & general purpose \\
\hline Agria & 1985 & GER & Quarta x Semio & processing \\
\hline Asterix & 1991 & NETH & Cardinal x VE- 70-9 & processing \\
\hline Caesar & 1990 & NETH & Monalisa x Ropta B1178 & processing \\
\hline Challenger & 2008 & NETH & Aziza x Victoria & processing \\
\hline Courage & 1998 & NETH & Lady Rosetta x HZ 81 H 202 & processing \\
\hline Crisps4all & 2008 & NETH & RZ 85-238 x RZ 87-44 & processing \\
\hline Felsina & 1992 & NETH & Morene x Gloria & processing \\
\hline Hermes & 1973 & AUT & DDR $5158 \times$ Sw 163/55 & processing \\
\hline Innovator & 1999 & NETH & Shepody x RZ 84-2580 & processing \\
\hline Lady Claire & 1996 & NETH & Agria x KW 78-34-470 & processing \\
\hline LadyRosetta & 1988 & NETH & Cardinal x VTN 62-33-3 & processing \\
\hline Leonardo & 1994 & NETH & Edzina x Ropta D 540 & Processing \\
\hline Maris Piper & 1963 & GB & Y 22/6 (Arran cairn x Herald & Processing \\
\hline Premiere & 1979 & NETH & Civa $x$ Provita & Processing \\
\hline Russet Burbank & 1908 & USA & Mutant of burbank & Processing \\
\hline Santana & 1994 & NETH & Spunta x Vk 69-491 & Processing \\
\hline Saturna & 1964 & NETH & Maritta x (Re cord x CPC 1673(adg)) & Processing \\
\hline Shepody & 1980 & CAN & Bake king x F58050 & Processing \\
\hline Taurus & 2008 & NETH & Panda x RZ 87-44 & Processing \\
\hline Triplo & 2000 & NETH & Agria $\mathrm{x}$ fresco & Processing \\
\hline Voyager & 2003 & NETH & RZ 85-238 x Oblelix & Processing \\
\hline VR 808 & 2009 & NETH & Lady Claire x Atlantic & Processing \\
\hline Kuras & 1996 & NETH & BRDA (= PG 285) x VK 69-491 & Starch industry \\
\hline
\end{tabular}

Year $=$ year of release, AUT $=$ Austria, $\mathrm{CAN}=$ Canada, $\mathrm{GB}=$ Great Brtain, FRA $=$ France, GER $=$ Germnay, NETH = Netherlands, USA = United States of America 
Supplemntary Table 2 Analysis of variance for 22 traits of the association panel at different $\mathrm{N}$ levels and locations

\begin{tabular}{|c|c|c|c|c|c|c|c|}
\hline Traits & N-level & Location & Genotype & N x Loc & $\mathrm{G} \times \mathrm{N}$ & Gx L & N x Loc $\times G$ \\
\hline DTE & ns & **** & $* * *$ & ns & $*$ & ns & ns \\
\hline PH & $* * *$ & $* * *$ & $* * *$ & ns & ns & $\mathrm{ns}$ & $\mathrm{ns}$ \\
\hline SNPP & $*$ & $* *$ & $* * *$ & ns & ns & $\mathrm{ns}$ & $\mathrm{ns}$ \\
\hline LCC & $*$ & $* * *$ & $* * *$ & $* * *$ & $* * *$ & ns & $\mathrm{ns}$ \\
\hline UCC & $*$ & $* * *$ & $* * *$ & ns & $* * *$ & ns & ns \\
\hline DTM & $\mathrm{ns}$ & $* * *$ & $*$ & ns & ns & $\mathrm{ns}$ & $\mathrm{ns}$ \\
\hline $\operatorname{tm} 1$ & ns & Ns & $* * *$ & ns & ns & $\mathrm{ns}$ & $\mathrm{ns}$ \\
\hline $\mathrm{t} 1$ & $\mathrm{~ns}$ & $* * *$ & $\mathrm{~ns}$ & $\mathrm{~ns}$ & $\mathrm{~ns}$ & $* * *$ & $\mathrm{~ns}$ \\
\hline $\mathrm{t} 2$ & ns & $* * *$ & $* * *$ & ns & ns & $\mathrm{ns}$ & $\mathrm{ns}$ \\
\hline te & ns & $* * *$ & $* * *$ & ns & $\mathrm{ns}$ & $\mathrm{ns}$ & $\mathrm{ns}$ \\
\hline$V \max$ & $* * *$ & $* * *$ & $* * *$ & $* * *$ & ns & $* * *$ & $\mathrm{~ns}$ \\
\hline $\mathrm{t} 2-\mathrm{t} 1$ & $* *$ & $* * *$ & $*$ & $*$ & ns & $\mathrm{ns}$ & $\mathrm{ns}$ \\
\hline te-t2 & $*$ & $* * *$ & $* * *$ & $*$ & $\mathrm{~ns}$ & ns & ns \\
\hline AP1 & $* *$ & $* * *$ & $* * *$ & $* * *$ & ns & $\mathrm{ns}$ & $\mathrm{ns}$ \\
\hline AP2 & $* * *$ & $* * *$ & $* * *$ & ns & $\mathrm{ns}$ & $\mathrm{ns}$ & $\mathrm{ns}$ \\
\hline AP3 & $* *$ & $* * *$ & $* * *$ & $\mathrm{~ns}$ & ns & $* * *$ & $\mathrm{~ns}$ \\
\hline AUC & $* * *$ & $* * *$ & $* * *$ & ns & ns & $* * *$ & $\mathrm{~ns}$ \\
\hline TYPP & $* * *$ & $* * *$ & $* * *$ & $* * *$ & ns & $* * *$ & $\mathrm{~ns}$ \\
\hline TNPP & $* * *$ & $* * *$ & $* * *$ & $\mathrm{~ns}$ & ns & $* *$ & $\mathrm{~ns}$ \\
\hline ATW & $* * *$ & $* *$ & $* * *$ & $\mathrm{~ns}$ & ns & $\mathrm{ns}$ & $\mathrm{ns}$ \\
\hline TDM\% & $* * *$ & $* * *$ & $* * *$ & $* * *$ & $\mathrm{~ns}$ & $\mathrm{~ns}$ & $\mathrm{~ns}$ \\
\hline NUE & $* * *$ & $* * *$ & $* *$ & $* * *$ & $\mathrm{~ns}$ & $* *$ & $\mathrm{~ns}$ \\
\hline
\end{tabular}

${ }^{*}=\mathrm{P} \leq 0.05,{ }^{* *}=\mathrm{P} \leq 0.01,{ }^{* * *}=\mathrm{P} \leq 0.001, \mathrm{DTE}=$ Days to Emergence, $\mathrm{PH}=$ Plant height, SNPP $=$ Stem Number plant ${ }^{-1}, \mathrm{LCC}=$ Lower leaf chlorophyll, $\mathrm{UCC}=$ upper leaf chlorophyll, $\mathrm{DTM}=$ days to maturity, $\mathrm{tml}=$ Inflection point, $\mathrm{t} 1=$ time to reach maximum canopy cover, $\mathrm{t} 2=$ Onset of canopy senesced, te=canopy complete senesced, Vmax=Maximum canopy cover, t2-t $1=$ Duration for max canopy, te-t2= Duration for senescence, AP1 = Area for phase one, AP2 = Area for phase two, AP3 = Area for phase three, AUC $=$ Area under the canopy curve, TYPP $=$ tuber yield plant $^{-1}, \mathrm{TNPP}=$ tuber number plant $^{-1}, \mathrm{ATW}=$ average tuber weight, $\mathrm{TDM} \%=$ Tuber dry matter $\%$, NUE $=$ nitrogen use efficiency, 
Supplementary Table 3. Summary statistics of the association mapping panel describing the variance component of various agronomic and physiological traits at each location and each production system under low $\mathrm{N}(\mathrm{LN})$ and high $\mathrm{N}(\mathrm{HN})$ conditions

\begin{tabular}{|c|c|c|c|c|c|c|c|c|c|}
\hline \multirow[t]{2}{*}{ Traits } & \multirow[t]{2}{*}{ Treat } & \multirow[t]{2}{*}{ Location } & \multicolumn{3}{|c|}{ Variance component (rainfed) } & \multirow[t]{2}{*}{ Location } & \multicolumn{3}{|c|}{ Variance component (Irrigation) } \\
\hline & & & $\sigma \mathrm{g}^{2}$ & $\sigma \mathrm{e}^{2}$ & $\mathrm{H}^{2}$ & & $\sigma \mathrm{g}^{2}$ & $\sigma \mathrm{e} 2$ & $\mathrm{H}^{2}$ \\
\hline \multirow[t]{4}{*}{$\mathrm{PH}$} & $\mathrm{LN}$ & DT & 8.3 & 18.3 & 0.31 & Koga & 27.3 & 16.04 & 0.6 \\
\hline & & INJ & 3.7 & 4.7 & 0.44 & INJ & 32.9 & 24.4 & 0.6 \\
\hline & $\mathrm{HN}$ & DT & 13.7 & 20 & 0.41 & Koga & 43.03 & 23.7 & 0.7 \\
\hline & & INJ & 13.6 & 6.7 & 0.7 & INJ & 48.6 & 24.2 & 0.7 \\
\hline \multirow[t]{4}{*}{ SNPP } & $\mathrm{LN}$ & DT & 0.6 & 0.5 & 0.5 & Koga & 0.08 & 0.6 & 0.1 \\
\hline & & INJ & 0.7 & 0.5 & 0.6 & INJ & 0.2 & 0.3 & 0.4 \\
\hline & $\mathrm{HN}$ & DT & 0.5 & 0.4 & 0.6 & Koga & 0.13 & 0.5 & 0.2 \\
\hline & & INJ & 0.8 & 0.5 & 0.6 & INJ & 0.2 & 0.3 & 0.3 \\
\hline \multirow[t]{4}{*}{ LCC } & $\mathrm{LN}$ & DT & 7.8 & 2.9 & 0.7 & Koga & 3.4 & 25.5 & 0.1 \\
\hline & & INJ & 5.34 & 5.8 & 0.5 & INJ & 8.7 & 19.7 & 0.3 \\
\hline & HN & DT & 16.21 & 1.7 & 0.9 & Koga & 8.3 & 15.7 & 0.4 \\
\hline & & INJ & 7.32 & 7.5 & 0.5 & INJ & 6.08 & 25 & 0.2 \\
\hline \multirow[t]{4}{*}{ UCC } & $\mathrm{LN}$ & DT & 6.24 & 2.6 & 0.7 & Koga & 4 & 16.7 & 0.2 \\
\hline & & INJ & 6.04 & 3.4 & 0.6 & INJ & 4.34 & 10.1 & 0.3 \\
\hline & HN & DT & 18.6 & 1.4 & 0.9 & Koga & 6.3 & 8.3 & 0.4 \\
\hline & & INJ & 6.6 & 6.4 & 0.5 & INJ & 3.9 & 14.3 & 0.2 \\
\hline \multirow[t]{4}{*}{ DTM } & $\mathrm{LN}$ & DT & 14.6 & 43.8 & 0.3 & Koga & 22.8 & 23.9 & 0.5 \\
\hline & & INJ & 2.6 & 40.3 & 0.06 & INJ & 8.4 & 179.2 & 0.05 \\
\hline & $\mathrm{HN}$ & DT & 16.02 & 26.8 & 0.4 & Koga & 12.2 & 31.4 & 0.3 \\
\hline & & INJ & 17 & 22 & 0.44 & INJ & 12.7 & 170.1 & 0.07 \\
\hline \multirow[t]{4}{*}{ TYPP } & $\mathrm{LN}$ & DT & 0.003 & 0.003 & 0.5 & Koga & 0.01 & 0.03 & 0.2 \\
\hline & & INJ & 0.001 & 0.001 & 0.4 & INJ & 0.005 & 0.004 & 0.7 \\
\hline & $\mathrm{HN}$ & DT & 0.01 & 0.005 & 0.6 & Koga & 0.01 & 0.1 & 0.1 \\
\hline & & INJ & 0.01 & 0.004 & 0.6 & INJ & 0.01 & 0.005 & 0.6 \\
\hline \multirow[t]{4}{*}{ TNPP } & LN & DT & 2.8 & 1.2 & 0.7 & Koga & 2.2 & 3 & 0.4 \\
\hline & & INJ & 1 & 0.5 & 0.7 & INJ & 0.9 & 0.42 & 0.7 \\
\hline & $\mathrm{HN}$ & DT & 3.6 & 1.8 & 0.7 & Koga & 1.3 & 13.5 & 0.1 \\
\hline & & INJ & 2.02 & 1.12 & 0.6 & INJ & 0.9 & 1 & 0.5 \\
\hline \multirow[t]{2}{*}{ ATW } & $\mathrm{LN}$ & DT & 106.9 & 90.4 & 0.5 & Koga & 472.05 & 609.4 & 0.4 \\
\hline & & INJ & 113.5 & 82.08 & 0.6 & INJ & 406.3 & 430.9 & 0.5 \\
\hline
\end{tabular}




\begin{tabular}{|c|c|c|c|c|c|c|c|c|c|}
\hline \multirow[t]{2}{*}{ Traits } & \multirow[t]{2}{*}{ Treat } & \multirow[t]{2}{*}{ Location } & \multicolumn{3}{|c|}{ Variance component (rainfed) } & \multirow[t]{2}{*}{ Location } & \multicolumn{3}{|c|}{ Variance component (Irrigation) } \\
\hline & & & $\sigma g^{2}$ & $\sigma \mathrm{e}^{2}$ & $\mathrm{H}^{2}$ & & $\sigma \mathrm{g}^{2}$ & $\sigma \mathrm{e} 2$ & $\mathrm{H}^{2}$ \\
\hline \multirow{6}{*}{ SG } & $\mathrm{HN}$ & DT & 411.6 & 126 & 0.8 & Koga & 95 & 1116 & 0.1 \\
\hline & & INJ & 182.5 & 128.9 & 0.6 & INJ & 444.4 & 376.8 & 0.5 \\
\hline & $\mathrm{LN}$ & DT & 0.0001 & 0.0002 & 0.4 & Koga & $6.32 \mathrm{E}-05$ & 0.0003 & 0.2 \\
\hline & & INJ & 0.0001 & 0.0003 & 0.2 & INJ & 0.000023 & 0.0003 & 0.08 \\
\hline & $\mathrm{HN}$ & DT & 0.0001 & 0.00012 & 0.43 & Koga & 0.000016 & 0.0002 & 0.1 \\
\hline & & INJ & 0.0001 & 0.00007 & 0.6 & INJ & 0.000043 & 0.0003 & 0.2 \\
\hline \multirow[t]{4}{*}{ TDM\% } & $\mathrm{LN}$ & DT & 5.3 & 7.4 & 0.4 & Koga & 3.01 & 14 & 0.2 \\
\hline & & INJ & 3 & 15.9 & 0.2 & INJ & 1.08 & 11.7 & 0.08 \\
\hline & $\mathrm{HN}$ & DT & 4.2 & 5.5 & 0.43 & Koga & 0.8 & 10.7 & 0.1 \\
\hline & & INJ & 5.7 & 3.5 & 0.6 & INJ & 2.05 & 11.7 & 0.15 \\
\hline \multirow[t]{4}{*}{ NUE } & $\mathrm{LN}$ & DT & 72 & 93.43 & 0.44 & Koga & 250.5 & 1723 & 0.13 \\
\hline & & INJ & 43 & 108.5 & 0.3 & INJ & 121.9 & 194.4 & 0.4 \\
\hline & $\mathrm{HN}$ & DT & 9.3 & 19 & 0.33 & Koga & 1.5 & 566.9 & 0.003 \\
\hline & & INJ & 8.8 & 17.2 & 0.34 & INJ & 24.4 & 34.9 & 0.4 \\
\hline \multirow[t]{4}{*}{$\mathrm{tm} 1$} & $\mathrm{LN}$ & DT & 0.7 & 4.6 & 0.12 & Koga & 3.04 & 7.6 & 0.3 \\
\hline & & INJ & 0.2 & 4.5 & 0.03 & INJ & 2.5 & 16.6 & 0.13 \\
\hline & $\mathrm{HN}$ & DT & 0.7 & 1.7 & 0.3 & Koga & 1 & 11.8 & 0.1 \\
\hline & & INJ & 0.8 & 2.4 & 0.3 & INJ & 0.9 & 45.9 & 0.02 \\
\hline \multirow[t]{4}{*}{$\mathrm{t} 1$} & $\mathrm{LN}$ & DT & 0.6 & 3.2 & 0.2 & Koga & 0.6 & 12.24 & 0.05 \\
\hline & & INJ & 0.4 & 5.7 & 0.07 & INJ & 4.3 & 19.8 & 0.2 \\
\hline & $\mathrm{HN}$ & DT & 0.4 & 3.2 & 0.1 & Koga & 3.22 & 11.02 & 0.2 \\
\hline & & INJ & 0.9 & 2.6 & 0.3 & INJ & 0.7 & 45.5 & 0.02 \\
\hline \multirow[t]{4}{*}{$\mathrm{t} 2$} & $\mathrm{LN}$ & DT & 0.7 & 5.6 & 0.1 & Koga & 2.5 & 13.4 & 0.2 \\
\hline & & INJ & 1.5 & 9.2 & 0.14 & INJ & 0.11 & 13.21 & 0.01 \\
\hline & $\mathrm{HN}$ & DT & 0.5 & 4.04 & 0.1 & Koga & 0.8 & 18.7 & 0.04 \\
\hline & & INJ & 0.7 & 4.2 & 0.14 & INJ & 7.9 & 10.01 & 0.4 \\
\hline \multirow[t]{4}{*}{ te } & LN & DT & 0.9 & 4.8 & 0.2 & Koga & 4 & 4.21 & 0.5 \\
\hline & & INJ & 2.8 & 5.7 & 0.33 & INJ & 0.02 & 3.6 & 0.01 \\
\hline & $\mathrm{HN}$ & DT & 0.5 & 6.32 & 0.08 & Koga & 2.21 & 6.8 & 0.3 \\
\hline & & INJ & 1.8 & 6.6 & 0.22 & INJ & 0.8 & 4.08 & 0.2 \\
\hline \multirow[t]{2}{*}{ Vmax } & LN & DT & 18 & 103.3 & 0.15 & Koga & 45.6 & 129.2 & 0.3 \\
\hline & & INJ & 8.6 & 34.5 & 0.2 & INJ & 82.33 & 50.14 & 0.6 \\
\hline
\end{tabular}




\begin{tabular}{|c|c|c|c|c|c|c|c|c|c|}
\hline \multirow[t]{2}{*}{ Traits } & \multirow[t]{2}{*}{ Treat } & \multirow[t]{2}{*}{ Location } & \multicolumn{3}{|c|}{ Variance component (rainfed) } & \multirow[t]{2}{*}{ Location } & \multicolumn{3}{|c|}{ Variance component (Irrigation) } \\
\hline & & & $\sigma \mathrm{g}^{2}$ & $\sigma \mathrm{e}^{2}$ & $\overline{\mathrm{H}^{2}}$ & & $\sigma \mathrm{g}^{2}$ & $\sigma \mathrm{e} 2$ & $\mathrm{H}^{2}$ \\
\hline & $\mathrm{HN}$ & DT & 62.5 & 91.01 & 0.4 & Koga & 67.3 & 163.2 & 0.3 \\
\hline & & INJ & 29.7 & 34.01 & 0.5 & INJ & 98.8 & 127.2 & 0.4 \\
\hline \multirow[t]{4}{*}{$\mathrm{t} 2-\mathrm{t} 1$} & $\mathrm{LN}$ & DT & 1.6 & 8.2 & 0.2 & Koga & 0.7 & 13.23 & 0.05 \\
\hline & & INJ & 2.9 & 9.8 & 0.23 & INJ & 8.9 & 23.22 & 0.3 \\
\hline & $\mathrm{HN}$ & DT & 0.05 & 5.7 & 0.01 & Koga & 2.2 & 14.6 & 0.1 \\
\hline & & INJ & 0.6 & 5.2 & 0.11 & INJ & 1.2 & 48.01 & 0.02 \\
\hline \multirow[t]{4}{*}{ te-t2 } & LN & DT & 2.4 & 11.06 & 0.2 & Koga & 6.16 & 18.5 & 0.3 \\
\hline & & INJ & 0.8 & 17.7 & 0.04 & INJ & 2.5 & 8.7 & 0.2 \\
\hline & HN & DT & 0.3 & 9.4 & 0.03 & Koga & 4.94 & 27.3 & 0.2 \\
\hline & & INJ & 2.8 & 10.8 & 0.2 & INJ & 6.9 & 8.4 & 0.5 \\
\hline \multirow[t]{4}{*}{ AP1 } & LN & DT & 2488.5 & 14791 & 0.14 & Koga & 14667.5 & 46811 & 0.2 \\
\hline & & INJ & 918.5 & 13508 & 0.06 & INJ & 8328 & 7350 & 0.5 \\
\hline & $\mathrm{HN}$ & DT & 4475 & 17360 & 0.21 & Koga & 18257 & 92252 & 0.2 \\
\hline & & INJ & 5302 & 11435 & 0.31 & INJ & 12731 & 15188 & 0.5 \\
\hline \multirow[t]{4}{*}{ AP2 } & LN & DT & 1997.5 & 17164 & 0.1 & Koga & 1764.5 & 35533 & 0.05 \\
\hline & & INJ & 4863.5 & 11999 & 0.3 & INJ & 22534 & 27658 & 0.5 \\
\hline & HN & DT & 1471 & 23405 & 0.06 & Koga & 5899.5 & 48249 & 0.11 \\
\hline & & INJ & 3218.5 & 16968 & 0.2 & INJ & 18723 & 78112 & 0.2 \\
\hline \multirow[t]{4}{*}{ AP3 } & $\mathrm{LN}$ & DT & 3371.5 & 27805 & 0.11 & Koga & 13189 & 57013 & 0.2 \\
\hline & & INJ & 472 & 15449 & 0.03 & INJ & 5437.5 & 11730 & 0.3 \\
\hline & $\mathrm{HN}$ & DT & 4415 & 32181 & 0.12 & Koga & 25015.5 & 83525 & 0.2 \\
\hline & & INJ & 7478 & 23366 & 0.24 & INJ & 12993 & 13695 & 0.5 \\
\hline \multirow[t]{4}{*}{ AUC } & $\mathrm{LN}$ & DT & 13902 & 37029 & 0.3 & Koga & 46107 & 116844 & 0.2 \\
\hline & & INJ & 7314 & 24053 & 0.23 & INJ & 64742 & 48676 & 0.6 \\
\hline & HN & DT & 31673.5 & 49773 & 0.4 & Koga & 65638 & 165774 & 0.3 \\
\hline & & INJ & 22312 & 31675 & 0.4 & INJ & 67995.5 & 118484 & 0.4 \\
\hline
\end{tabular}

DT $=$ Debre-Tabor, INJ $=$ Injibara, PH $=$ Plant height, SNPP $=$ Stem Number plant ${ }^{-1}$, LCC $=$ Lower leaf chlorophyll, UCC $=$ upper leaf chlorophyll, DTM $=$ days to maturity, TYPP $=$ tuber yield plant ${ }^{-1}, \mathrm{TNPP}=$ tuber number plant ${ }^{-1}, \mathrm{ATW}=$ average tuber weight, $\mathrm{SG}=$ specific gravity, $\mathrm{TDM} \%=$ Tuber dry matter $\%, \mathrm{NUE}=$ nitrogen use efficiency, $\mathrm{tml}=$ Inflection point, $\mathrm{t} 1=$ time to reach maximum canopy cover, $\mathrm{t} 2$ = Onset of canopy senesced, te = canopy complete senesced, Vmax = Maximum canopy cover, $\mathrm{t} 2$-t $1=$ Duration for max canopy, te-t $2=$ Duration for senescence, $\mathrm{AP} 1=$ Area for phase one, $\mathrm{AP} 2=$ Area for phase two $\mathrm{AP} 3=$ Area for phase three, $\mathrm{AUC}=$ Area under the canopy curve, $\mathrm{LN}=$ low $\mathrm{N}\left(40 \mathrm{~kg} \mathrm{ha}^{-1}, \mathrm{HN}=\right.$ high $\mathrm{N}\left(120 \mathrm{~kg} \mathrm{ha}^{-1}\right), \mathrm{DT}=$ Debre-Tabor, INJ = Injibara $\sigma \mathrm{g}^{2}=$ genotypic variance,$\sigma \mathrm{e}^{2}=$ environmental variance, $\mathrm{H}^{2}=$ broad sense heritability 
Supplementary Table 4. Summary statistics of the association mapping panel describing the variance component of location combined agronomic and physiological traits at each production system under low $\mathrm{N}(\mathrm{LN})$ and high $\mathrm{N}(\mathrm{HN})$ conditions

\begin{tabular}{|c|c|c|c|c|c|c|c|c|c|}
\hline \multirow[t]{2}{*}{ Traits } & \multirow[t]{2}{*}{ Treat } & \multicolumn{4}{|c|}{ Variance component (rainfed) } & \multicolumn{4}{|c|}{ Variance component (irrigation) } \\
\hline & & $\sigma \mathrm{g}^{2}$ & $\sigma \mathrm{ge}^{2}$ & $\sigma \mathrm{e} 2$ & $\mathrm{H}^{2}$ & $\sigma \mathrm{g}^{2}$ & $\sigma \mathrm{ge}^{2}$ & $\sigma \mathrm{e}^{2}$ & $\mathrm{H}^{2}$ \\
\hline \multirow[t]{2}{*}{$\mathrm{PH}$} & $\mathrm{LN}$ & 20.02 & 0.5 & 11.2 & 0.6 & 116.5 & 4.8 & 21.5 & 0.8 \\
\hline & $\mathrm{HN}$ & 53 & -0.6 & 13.3 & 0.8 & 126.4 & 6.2 & 22.5 & 0.8 \\
\hline \multirow[t]{2}{*}{ SNPP } & $\mathrm{LN}$ & 3.7 & -0.3 & 0.6 & 0.9 & 0.5 & 0.0 & 0.5 & 0.5 \\
\hline & $\mathrm{HN}$ & 3.6 & -0.2 & 0.5 & 0.9 & 0.5 & 0.03 & 0.4 & 0.6 \\
\hline \multirow[t]{2}{*}{ LCC } & $\mathrm{LN}$ & 15.4 & 2.34 & 4.5 & 0.8 & 31.7 & -3.6 & 25.7 & 0.6 \\
\hline & $\mathrm{HN}$ & 9.7 & 8.7 & 4.2 & 0.6 & 28.9 & -0.11 & 20.4 & 0.6 \\
\hline \multirow[t]{2}{*}{ UCC } & $\mathrm{LN}$ & 14.9 & 2.5 & 3.33 & 0.8 & 21.5 & -1.6 & 14.5 & 0.6 \\
\hline & $\mathrm{HN}$ & 7.5 & 9 & 3.7 & 0.6 & 20.44 & 1.3 & 10.9 & 0.6 \\
\hline \multirow[t]{2}{*}{ DTM } & $\mathrm{LN}$ & 38.5 & 0.9 & 33.3 & 0.5 & 22.4 & 4.4 & 103.8 & 0.2 \\
\hline & $\mathrm{HN}$ & 60.3 & 2.31 & 22.5 & 0.7 & 58.9 & 2 & 104.3 & 0.4 \\
\hline \multirow[t]{2}{*}{ TYPP } & $\mathrm{LN}$ & 5104 & 289.5 & 2281 & 0.7 & 23279 & 383 & 17861 & 0.6 \\
\hline & $\mathrm{HN}$ & 21362 & 361 & 4358 & 0.7 & 22315 & 2056 & 52994 & 0.3 \\
\hline \multirow[t]{2}{*}{ TNPP } & $\mathrm{LN}$ & 5.3 & 0.4 & 0.9 & 0.8 & 5.6 & 0.1 & 1.9 & 0.7 \\
\hline & $\mathrm{HN}$ & 8.31 & 0.5 & 1.4 & 0.8 & 2.64 & -0.8 & 6.3 & 0.31 \\
\hline \multirow[t]{2}{*}{ ATW } & $\mathrm{LN}$ & 420.8 & -2.9 & 78.05 & 0.8 & 1586.7 & 19.6 & 548.7 & 0.7 \\
\hline & $\mathrm{HN}$ & 637.6 & 8.9 & 106.9 & 0.9 & 337.2 & 118.5 & 777.9 & 0.3 \\
\hline \multirow[t]{2}{*}{ SG } & $\mathrm{LN}$ & 0.0 & 0.0 & 0.0 & 0.6 & 0.0 & 0.0 & 0.0 & 0.13 \\
\hline & $\mathrm{HN}$ & 0.0 & 0.0 & 0.0 & 0.01 & 0.0 & 0.0 & 0.0 & 0.08 \\
\hline \multirow[t]{2}{*}{ TDM\% } & LN & 17.7 & -1.44 & 12.02 & 0.6 & 2.14 & 1 & 14.4 & 0.13 \\
\hline & $\mathrm{HN}$ & 19.9 & 0.14 & 3.9 & 0.8 & 1.3 & -1.8 & 15.6 & 0.08 \\
\hline \multirow[t]{2}{*}{ NUE } & $\mathrm{LN}$ & 165.6 & 2.4 & 105.8 & 0.6 & 367.0 & -143.5 & 1388 & 0.22 \\
\hline & $\mathrm{HN}$ & 41.5 & 0.7 & 17.6 & 0.7 & 20.0 & -27.5 & 351.9 & 0.06 \\
\hline \multirow[t]{2}{*}{$\mathrm{tm} 1$} & $\mathrm{LN}$ & 2.5 & -1.23 & 8.5 & 0.24 & 1.27 & 1.13 & 7.4 & 0.14 \\
\hline & $\mathrm{HN}$ & 0.6 & 1.4 & 2.12 & 0.2 & 1.9 & -3.9 & 23.5 & 0.08 \\
\hline
\end{tabular}




\begin{tabular}{|c|c|c|c|c|c|c|c|c|c|}
\hline \multirow[t]{2}{*}{ Traits } & \multirow[t]{2}{*}{ Treat } & \multicolumn{4}{|c|}{ Variance component (rainfed) } & \multicolumn{4}{|c|}{ Variance component (irrigation) } \\
\hline & & $\sigma g^{2}$ & $\sigma g \mathrm{e}^{2}$ & $\sigma \mathrm{e} 2$ & $\mathrm{H}^{2}$ & $\sigma g^{2}$ & $\sigma \mathrm{ge}^{2}$ & $\sigma \mathrm{e}^{2}$ & $\mathrm{H}^{2}$ \\
\hline \multirow[t]{2}{*}{$\mathrm{t} 1$} & $\mathrm{LN}$ & 1.6 & -0.3 & 4.22 & 0.3 & 2.8 & -0.64 & 11.7 & 0.2 \\
\hline & $\mathrm{HN}$ & 1 & 0.43 & 3.02 & 0.24 & 6.4 & -1.8 & 17.43 & 0.3 \\
\hline \multirow[t]{2}{*}{$\mathrm{t} 2$} & $\mathrm{LN}$ & 4.6 & -0.4 & 6.7 & 0.41 & 5.6 & 0.05 & 12.33 & 0.3 \\
\hline & $\mathrm{HN}$ & 0.7 & 0.5 & 3.9 & 0.2 & 5.8 & 2.3 & 12.5 & 0.3 \\
\hline \multirow[t]{2}{*}{$\mathrm{Te}$} & $\mathrm{LN}$ & 4.8 & 0.6 & 5.4 & 0.5 & 5.07 & 1.2 & 3.8 & 0.5 \\
\hline & $\mathrm{HN}$ & 4.8 & -0.07 & 6.7 & 0.42 & 3.0 & 0.42 & 5.02 & 0.4 \\
\hline \multirow[t]{2}{*}{ Vmax } & $\mathrm{LN}$ & 55.1 & -9.24 & 83.34 & 0.4 & 212 & 17.8 & 96 & 0.7 \\
\hline & HN & 307.2 & 4000.7 & 60.7 & 0.7 & 367.5 & -2.6 & 163.4 & 0.7 \\
\hline \multirow[t]{2}{*}{$\mathrm{t} 2-\mathrm{t} 1$} & $\mathrm{LN}$ & 2.8 & -0.04 & 8.9 & 0.24 & 3.9 & 1.32 & 11.5 & 0.24 \\
\hline & $\mathrm{HN}$ & 1.5 & 0.3 & 5.21 & 0.22 & 3.4 & 2.2 & 15.8 & 0.2 \\
\hline \multirow[t]{2}{*}{ te-t 2} & $\mathrm{LN}$ & 4.5 & -1.4 & 13.5 & 0.3 & 6.7 & 2.7 & 14.2 & 0.3 \\
\hline & $\mathrm{HN}$ & 1 & 0.98 & 10.6 & 0.08 & 8.42 & 3.60 & 14.7 & 0.3 \\
\hline \multirow[t]{2}{*}{ AP1 } & $\mathrm{LN}$ & 8204 & -1324.5 & 143950 & 0.4 & 19221 & 7015.5 & 26995 & 0.4 \\
\hline & HN & 3295 & 3513.5 & 146960 & 0.18 & 20518 & 10538 & 48180 & 0.3 \\
\hline \multirow[t]{2}{*}{ AP2 } & $\mathrm{LN}$ & 6164 & 163.5 & 14575 & 0.42 & 14025 & 7510 & 28433 & 0.3 \\
\hline & $\mathrm{HN}$ & 2433 & 2794 & 19697 & 0.11 & 30430 & 10957.5 & 53654 & 0.3 \\
\hline \multirow[t]{2}{*}{ AP3 } & $\mathrm{LN}$ & 9036 & -4519.5 & 23214 & 0.5 & 31802 & 2922 & 35243 & 0.5 \\
\hline & HN & 24742 & 298.5 & 28703 & 0.5 & 47678 & 13293.5 & 42419 & 0.5 \\
\hline \multirow[t]{2}{*}{ AUC } & $\mathrm{LN}$ & 32332 & 1262.5 & 31924 & 0.5 & 203150 & 16425.5 & 86607 & 0.7 \\
\hline & HN & 101040 & 478 & 387170 & 0.7 & 314597 & 8451.5 & 143815 & 0.7 \\
\hline
\end{tabular}

$\mathrm{PH}=$ Plant height, SNPP = Stem Number per plant, LCC = Lower leaf chlorophyll, UCC= upper leaf chlorophyll, DTM= days to maturity, TYPP=tuber yield per plant, TNPP= tuber number per plant, ATW = average tuber weight, $\mathrm{SG}=$ specific gravity, TDM\%= Tuber dry matter\%, NUE= nitrogen use efficiency, $\mathrm{tml}=$ Inflection point, $\mathrm{t} 1=$ time to reach maximum canopy cover, $\mathrm{t} 2=\mathrm{Onset}$ of canopy senesced, te=canopy complete senesced, Vmax=Maximum canopy cover, $\mathrm{t} 2-\mathrm{t} 1=$ Duration for max canopy, te-t2 $=$ Duration for senescence, $\mathrm{AP} 1=$ Area for phase one, $\mathrm{AP} 2=\mathrm{Area}$ for $\mathrm{phase} \mathrm{two}, \mathrm{AP} 3=\mathrm{Area}$ for phase three, $\mathrm{AUC}=\mathrm{Area}$ under the canopy curve, $\mathrm{LN}=$ low $\mathrm{N}\left(40 \mathrm{~kg} \mathrm{ha}^{-1}\right), \mathrm{HN}=$ high $\mathrm{N}\left(120 \mathrm{~kg} \mathrm{ha}^{-1}\right), \sigma \mathrm{g}^{2}=$ genotypic variance, $\sigma \mathrm{ge} 2=$ genotype $\mathrm{x}$ environement interaction variance, $\sigma \mathrm{e} 2=$ environmental variance, $\mathrm{H}^{2}=$ broad sense heritability 
Supplementary Table 5. Marker trait associations significant at $-\log _{10}(\mathrm{P})=0.05$ for NUE and NUE related agronomic and physiological traits measured in 15 environments

\begin{tabular}{|c|c|c|c|c|c|c|c|c|}
\hline Traits & Environments & Model & Marker & Chrom & Position & Allele.freq & $-\log _{10}(\mathrm{P})$ & Effect \\
\hline \multirow[t]{6}{*}{ AP2 } & DTLN13 & 2-dom-ref & solcap_snp_c2_9172 & VI & 58844975 & 0.31 & 5.58 & 162.2 \\
\hline & HN combined & 1-dom-ref & solcap_snp_c2_45179 & VII & 46814225 & 0.63 & 4.62 & 120.07 \\
\hline & HN combined 15 & 1-dom-ref & solcap_snp_c2_45567 & IX & 48735918 & 0.77 & 4.54 & 174.82 \\
\hline & IBLN13 & General & solcap_snp_c1_10144 & XI & 10767532 & 0.24 & 5.39 & NA \\
\hline & LN combined & Additive & solcap_snp_c2_5078 & I & 80591939 & 0.48 & 5.26 & 35.43 \\
\hline & Over combined & 1-dom-ref & solcap_snp_c2_45179 & VII & 46814225 & 0.63 & 4.52 & 89.78 \\
\hline AP3 & IBHN13 & 2-dom-alt & PotVar0087318 & IV & 66212305 & 0.61 & 5.31 & 178.08 \\
\hline \multirow[t]{4}{*}{ AUC } & IBHN13 & 2-dom-ref & PotVar0010985 & IX & 57166420 & 0.80 & 5.55 & -245.89 \\
\hline & IBLN13 & 1-dom-alt & solcap_snp_c2_33517 & V & 1413732 & 0.18 & 5.78 & -153.08 \\
\hline & LN combined & 2-dom-alt & PotVar0019456 & III & 2742393 & 0.28 & 6.88 & -202.9 \\
\hline & LN combined 15 & 2-dom-alt & PotVar0019456 & III & 2742393 & 0.28 & 5.36 & -349.06 \\
\hline \multirow[t]{4}{*}{ DTE } & DTHN13 & 1-dom-ref & PotVar0127165 & VI & 42867728 & 0.59 & 4.84 & 2.56 \\
\hline & IBHN13 & Additive & PotVar0060758 & I & 82029061 & 0.46 & 5.36 & 0.85 \\
\hline & LN combined 13 & 1-dom-ref & solcap_snp_c2_23929 & XI & 6098398 & 0.82 & 5.08 & 1.64 \\
\hline & IBHN15 & General & PotVar0064473 & XI & 787383 & 0.54 & 5.69 & NA \\
\hline \multirow[t]{4}{*}{ DTM } & DTLN13 & 2-dom-alt & PotVar0043347 & I & 69910940 & 0.75 & 5.29 & 10.54 \\
\hline & DTLN13 & General & PotVar0075255 & IV & 67807068 & 0.55 & 6.25 & NA \\
\hline & IBHN13 & 2-dom-ref & PotVar0010985 & IX & 57166420 & 0.80 & 4.97 & -7.09 \\
\hline & IBLN13 & General & solcap_snp_c1_7132 & III & 54314873 & 0.36 & 7.28 & NA \\
\hline \multirow[t]{3}{*}{ NUE } & DTHN13 & 2-dom-alt & PotVar0040680 & VI & 52929083 & 0.21 & 5.12 & 5.68 \\
\hline & LN combined 13 & 2-dom-alt & PotVar0019302 & III & 2235688 & 0.13 & 5.41 & 15.39 \\
\hline & LN combined 13 & 2-dom-alt & PotVar0026355 & V & 4335324 & 0.10 & 5.15 & 17.72 \\
\hline \multirow[t]{4}{*}{ SNPP } & DTHN13 & 2-dom-ref & PotVar0116800 & $\mathrm{X}$ & 1910636 & 0.45 & 5.01 & 1.09 \\
\hline & HN combined & Additive & PotVar0014376 & V & 12237074 & 0.17 & 5.49 & -0.39 \\
\hline & HN combined & 2-dom-ref & PotVar0116800 & $\mathrm{X}$ & 1910636 & 0.46 & 5.75 & 0.71 \\
\hline & HN combined 13 & 2-dom-ref & PotVar0041871 & I & 75179906 & 0.36 & 5.94 & 1.48 \\
\hline
\end{tabular}




\begin{tabular}{|c|c|c|c|c|c|c|c|c|}
\hline Traits & Environments & Model & Marker & Chrom & Position & Allele.freq & $-\log _{10}(\mathrm{P})$ & Effect \\
\hline & IBHN13 & General & PotVar0090060 & II & 41787809 & 0.09 & 5.54 & NA \\
\hline & IBHN13 & 2-dom-ref & PotVar0116800 & $\mathrm{X}$ & 1910636 & 0.45 & 4.86 & 1.21 \\
\hline & KOLN15 & 1-dom-ref & PotVar0097439 & VIII & 53835553 & 0.76 & 4.74 & -0.71 \\
\hline & Over combined & 2-dom-ref & PotVar0116817 & $\mathrm{X}$ & 1912160 & 0.42 & 5.22 & 0.64 \\
\hline \multirow[t]{3}{*}{ t1 } & DTHN13 & 2-dom-ref & PotVar0110053 & VIII & 3214131 & 0.36 & 4.98 & 1.97 \\
\hline & HN combined 13 & 2-dom-alt & PotVar0046416 & II & 36845853 & 0.54 & 7.07 & -1.53 \\
\hline & LN combined 13 & 1-dom-alt & PotVar0134786 & VIII & 41088438 & 0.31 & 6.49 & -1.84 \\
\hline \multirow[t]{3}{*}{$\mathrm{t} 2$} & HN combined 13 & 2-dom-alt & solcap_snp_c2_2721 & I & 58197448 & 0.60 & 6.22 & -1.46 \\
\hline & HN combined 15 & 1-dom-ref & PotVar0034950 & $\mathrm{V}$ & 50863328 & 0.75 & 4.77 & -2.75 \\
\hline & IBLN13 & General & solcap_snp_c2_57254 & III & 53433832 & 0.39 & 5.96 & NA \\
\hline \multirow[t]{6}{*}{$\mathrm{t} 2-\mathrm{t} 1$} & HN combined 13 & 2-dom-alt & PotVar0046416 & II & 36845853 & 0.54 & 5.4 & 1.44 \\
\hline & IBLN13 & 2-dom-ref & solcap_snp_c2_26015 & VI & 50155639 & 0.79 & 5.09 & -3.52 \\
\hline & KOHN15 & Additive & PotVar0056273 & III & 50890271 & 0.36 & 5.26 & 1.89 \\
\hline & KOHN15 & 2-dom-ref & PotVar0087064 & IV & 66147380 & 0.78 & 5.65 & -4.52 \\
\hline & KOHN15 & 2-dom-ref & solcap_snp_c2_55553 & VI & 2182330 & 0.30 & 5.21 & 6.52 \\
\hline & LN combined & 1-dom-ref & PotVar0014354 & $\mathrm{V}$ & 12658442 & 0.82 & 4.75 & -1.24 \\
\hline \multirow[t]{4}{*}{ TDM\% } & DTHN13 & General & PotVar0109750 & II & 11294543 & 0.12 & 5.35 & NA \\
\hline & HN combined & 2-dom-alt & PotVar0030875 & III & 55659310 & 0.13 & 5.4 & 2.77 \\
\hline & IBHN15 & 2-dom-ref & PotVar0091938 & $\mathrm{V}$ & 9824216 & 0.86 & 5.05 & -4.31 \\
\hline & IBLN13 & 1-dom-alt & PotVar0023375 & VIII & 56624935 & 0.10 & 5.14 & 3.56 \\
\hline \multirow[t]{3}{*}{ te } & DTLN13 & 2-dom-ref & PotVar0101916 & IX & 52407969 & 0.87 & 4.95 & 2.95 \\
\hline & LN combined & 1-dom-ref & PotVar0034688 & $\mathrm{V}$ & 51682609 & 0.62 & 4.95 & -2.05 \\
\hline & Over combined & General & solcap_snp_c2_23596 & IV & 641790 & 0.42 & 5.25 & NA \\
\hline te-t 2 & IBLN15 & 1-dom-alt & solcap_snp_c2_38643 & VII & 44288221 & 0.23 & 5.87 & -2.92 \\
\hline \multirow[t]{5}{*}{$\operatorname{tm} 1$} & HN combined & 2-dom-ref & PotVar0024747 & $\mathrm{V}$ & 2964094 & 0.86 & 5.18 & 2.19 \\
\hline & IBLN13 & 2-dom-alt & PotVar0127400 & VII & 9119537 & 0.39 & 5.76 & 1.7 \\
\hline & KOLN15 & 2-dom-ref & solcap_snp_c2_36941 & 0 & 0 & 0.42 & 5.19 & -5.17 \\
\hline & KOLN15 & 2-dom-alt & solcap_snp_c2_53708 & I & 44890251 & 0.71 & 5.18 & 4.33 \\
\hline & LN combined & General & solcap_snp_c2_39499 & IX & 49977704 & 0.62 & 5.36 & NA \\
\hline
\end{tabular}




\begin{tabular}{|c|c|c|c|c|c|c|c|c|}
\hline Traits & Environments & Model & Marker & Chrom & Position & Allele.freq & $-\log _{10}(\mathrm{P})$ & Effect \\
\hline \multirow{5}{*}{ TNPP } & LN combined & General & PotVar0008106 & XI & 40967802 & 0.41 & 5.42 & NA \\
\hline & DTHN13 & 1-dom-alt & PotVar0130496 & XI & 44430446 & 0.43 & 5.19 & -4.21 \\
\hline & HN combined & Additive & solcap_snp_c2_25261 & VII & 47674858 & 0.24 & 6.14 & 0.76 \\
\hline & HN combined 13 & 2-dom-alt & PotVar0009411 & II & 40336749 & 0.11 & 5.42 & 3.65 \\
\hline & IBLN15 & 1-dom-ref & solcap_snp_c2_50570 & II & 35399277 & 0.78 & 4.54 & 1.11 \\
\hline \multirow{9}{*}{ TYPP } & IBHN15 & 2-dom-alt & PotVar0040353 & VI & 53752438 & 0.15 & 5.19 & 2.15 \\
\hline & HN combined 13 & 1-dom-ref & solcap_snp_c2_5286 & III & 8564142 & 0.60 & 5.45 & 3.04 \\
\hline & HN combined 13 & 2-dom-ref & PotVar0052570 & XII & 59793350 & 0.88 & 6.17 & -3.22 \\
\hline & Over combined & Additive & solcap_snp_c2_25261 & VII & 47674858 & 0.24 & 5.99 & 0.65 \\
\hline & KOHN15 & 2-dom-ref & PotVar0121097 & IV & 12333285 & 0.35 & 5.27 & 402.84 \\
\hline & KOLN15 & 1-dom-ref & solcap_snp_c2_26796 & IV & 9524541 & 0.59 & 6.31 & 296.66 \\
\hline & KOLN15 & 1-dom-ref & PotVar0060022 & XI & 9377347 & 0.59 & 6.13 & 325.02 \\
\hline & KOLN15 & 2-dom-ref & PotVar0052374 & XI & 59957211 & 0.89 & 4.9 & -284 \\
\hline & LN combined 15 & 2-dom-ref & PotVar0102235 & I & 51293720 & 0.39 & 5.06 & 143.68 \\
\hline \multirow{5}{*}{ UCC } & LN combined 15 & 1-dom-ref & solcap_snp_c2_26796 & IV & 9524541 & 0.59 & 5.65 & 188.47 \\
\hline & LN combined 15 & General & PotVar0060022 & XI & 9377347 & 0.59 & 5.75 & NA \\
\hline & DTHN13 & 1-dom-ref & solcap_snp_c2_12216 & I & 72051529 & 0.64 & 4.78 & 5.26 \\
\hline & HN combined & 2-dom-alt & PotVar0011708 & IX & 2144492 & 0.25 & 5.09 & 2.42 \\
\hline & HN combined 13 & 2-dom-ref & PotVar0011173 & IX & 57422879 & 0.85 & 5.8 & 4.54 \\
\hline \multirow{3}{*}{ Vmax } & IBHN13 & Additive & PotVar0127625 & VI & 55908088 & 0.06 & 5.51 & -3.78 \\
\hline & DTLN13 & 1-dom-ref & PotVar0089284 & II & 26584179 & 0.69 & 4.91 & -9.9 \\
\hline & IBLN13 & Additive & PotVar0048670 & V & 351772 & 0.78 & 5.43 & -3.33 \\
\hline
\end{tabular}


Chaptar 5: Supplementary data

Supplementary Table 1. List of potato cultivars with release year, origin and purpose

\begin{tabular}{|c|c|c|c|}
\hline Cultivar name & Year & Origion & Purpose \\
\hline Adora & 1990 & NETH & fresh consumption \\
\hline Agata & 1990 & NETH & fresh consumption \\
\hline Agria & 1985 & GER & Processing Industry \\
\hline Almera & 1999 & NETH & fresh consumption \\
\hline Ambition & 2007 & $\mathrm{NETH}$ & fresh consumption \\
\hline Annabelle & 2001 & $\mathrm{NETH}$ & fresh consumption \\
\hline Arinda & 1993 & NETH & fresh consumption \\
\hline Arizona & 2009 & NETH & fresh consumption \\
\hline Asterix & 1991 & NETH & Processing Industry \\
\hline Bafana & 2009 & NETH & fresh consumption \\
\hline Baraka & 1971 & NETH & fresh consumption \\
\hline Bartina & 1988 & NETH & fresh consumption \\
\hline Bellini & 2001 & NETH & fresh consumption \\
\hline Berber & 1984 & NETH & fresh consumption \\
\hline Bintje & 1910 & NETH & fresh consumption \\
\hline Caesar & 1990 & NETH & Processing Industry \\
\hline Canberra & 2007 & NETH & General \\
\hline Carlita & 1991 & NETH & General \\
\hline Carrera & 1999 & NETH & General \\
\hline Challenger & 2008 & NETH & Processing Industry \\
\hline Charlotte & 1981 & FRA & fresh consumption \\
\hline Cleopatra & 1980 & NETH & General \\
\hline Colomba & 2011 & NETH & fresh consumption \\
\hline Compass & 2011 & NETH & General \\
\hline Courage & 1998 & NETH & Processing Industry \\
\hline Crisp4all & 2008 & NETH & Processing Industry \\
\hline Desiree & 1962 & NETH & fresh consumption \\
\hline Evora & 2011 & NETH & General \\
\hline Fabula & 1997 & NETH & fresh consumption \\
\hline Faluka & 2006 & NETH & fresh consumption \\
\hline Felsina & 1992 & NETH & Processing Industry \\
\hline
\end{tabular}




\begin{tabular}{|c|c|c|c|}
\hline Cultivar name & Year & Origion & Purpose \\
\hline Flamenco & 2013 & NETH & General \\
\hline Frisia & 1988 & NETH & General \\
\hline Hansa & 1957 & GER & fresh consumption \\
\hline Hermes & 1973 & AUT & Processing Industry \\
\hline Innovator & 1999 & NETH & Processing Industry \\
\hline Ivory Russet & 2011 & NETH & Processing Industry \\
\hline Jaerla & 1969 & NETH & fresh consumption \\
\hline Jazzy & 2010 & NETH & Processing Industry \\
\hline Kastelli & 2011 & NETH & fresh consumption \\
\hline Kennebec & 1948 & USA & ancient cultivar \\
\hline Kondor & 1984 & NETH & fresh consumption \\
\hline Kuras & 1996 & NETH & Starch industry \\
\hline Kuroda & 1998 & NETH & fresh consumption \\
\hline Lady Christl & 1996 & NETH & fresh consumption \\
\hline Lady Claire & 1996 & NETH & Processing Industry \\
\hline Lady Rosetta & 1988 & NETH & Processing Industry \\
\hline Leonardo & 1994 & NETH & Processing Industry \\
\hline Liseta & 1988 & NETH & fresh consumption \\
\hline Lucinda & 2011 & NETH & General \\
\hline Marabel & 1993 & NETH & fresh consumption \\
\hline Marfona & 1977 & NETH & fresh consumption \\
\hline Marilyn & 2006 & NETH & fresh consumption \\
\hline Maris Piper & 1963 & GB & Processing Industry \\
\hline Markies & 1997 & NETH & fresh consumption \\
\hline Melody & 2001 & NETH & General \\
\hline Memphis & 2012 & NETH & General \\
\hline Monalisa & 1982 & NETH & fresh consumption \\
\hline Mondial & 1987 & NETH & fresh consumption \\
\hline Mozart & 2003 & NETH & General \\
\hline Navigator & 2013 & NETH & Processing Industry \\
\hline Nicola & 1973 & GER & fresh consumption \\
\hline Orchestra & 2007 & NETH & fresh consumption \\
\hline Panther & 2011 & NETH & General \\
\hline
\end{tabular}




\begin{tabular}{|c|c|c|c|}
\hline Cultivar name & Year & Origion & Purpose \\
\hline Picasso & 1994 & NETH & fresh consumption \\
\hline Red Scarlett & 1999 & NETH & fresh consumption \\
\hline Rodeo & 1999 & NETH & General \\
\hline Ronaldo & 2011 & NETH & General \\
\hline Sagitta & 2006 & NETH & General \\
\hline Sifra & 2008 & NETH & General \\
\hline Spunta & 1968 & NETH & fresh consumption \\
\hline Sylvana & 2008 & NETH & General \\
\hline Taurus & 2008 & NETH & Processing Industry \\
\hline Timate & 1984 & NETH & fresh consumption \\
\hline Triplo & 2000 & NETH & Processing Industry \\
\hline Victoria & 1997 & NETH & General \\
\hline Vivaldi & 1998 & NETH & General \\
\hline Volumia & 2004 & NETH & General \\
\hline Voyager & 2003 & NETH & Processing Industry \\
\hline VR 808 & 2009 & NETH & Processing Industry \\
\hline Zina Red & 2013 & NETH & fresh consumption \\
\hline
\end{tabular}


Supplementary Table 2. Mean TYPP (kg/ha) of 81 potato cultivars (G1 to G81) tested across the test environments (E1 to E8)

\begin{tabular}{|c|c|c|c|c|c|c|c|c|c|c|}
\hline Code & Cultivar & E1 & E2 & E3 & E4 & E5 & E6 & E7 & E8 & Mean \\
\hline G1 & Adora & 0.34 & 0.49 & 0.22 & 0.46 & 0.25 & 0.30 & 0.41 & 1.38 & 0.5 \\
\hline $\mathrm{G} 2$ & Agata & 0.18 & 0.28 & 0.10 & 0.28 & 0.30 & 0.38 & 0.65 & 0.62 & 0.4 \\
\hline G3 & Agria & 0.30 & 0.42 & 0.22 & 0.43 & 0.37 & 0.44 & 0.67 & 0.58 & 0.4 \\
\hline G4 & Almera & 0.28 & 0.38 & 0.18 & 0.37 & 0.37 & 0.46 & 0.72 & 1.25 & 0.5 \\
\hline G5 & Ambition & 0.37 & 0.51 & 0.28 & 0.50 & 0.35 & 0.40 & 0.56 & 0.76 & 0.5 \\
\hline G6 & Annabelle & 0.20 & 0.34 & 0.12 & 0.33 & 0.24 & 0.30 & 0.50 & 0.51 & 0.3 \\
\hline G7 & Arinda & 0.32 & 0.45 & 0.24 & 0.45 & 0.35 & 0.41 & 0.61 & 0.76 & 0.5 \\
\hline G8 & Arizona & 0.37 & 0.51 & 0.28 & 0.50 & 0.37 & 0.42 & 0.60 & 0.71 & 0.5 \\
\hline G9 & Asterix & 0.36 & 0.53 & 0.27 & 0.52 & 0.28 & 0.31 & 0.42 & 0.69 & 0.4 \\
\hline G10 & Bafana & 0.29 & 0.39 & 0.19 & 0.38 & 0.39 & 0.47 & 0.73 & 1.04 & 0.5 \\
\hline G11 & Baraka & 0.29 & 0.44 & 0.21 & 0.43 & 0.29 & 0.34 & 0.51 & 0.58 & 0.4 \\
\hline G12 & Bartina & 0.30 & 0.45 & 0.22 & 0.44 & 0.28 & 0.33 & 0.48 & 0.63 & 0.4 \\
\hline G13 & Bellini & 0.29 & 0.44 & 0.20 & 0.43 & 0.26 & 0.31 & 0.46 & 0.73 & 0.4 \\
\hline G14 & Berber & 0.23 & 0.37 & 0.13 & 0.36 & 0.21 & 0.27 & 0.43 & 0.94 & 0.4 \\
\hline G15 & Bintje & 0.26 & 0.37 & 0.17 & 0.36 & 0.35 & 0.43 & 0.68 & 0.92 & 0.4 \\
\hline G16 & Caesar & 0.34 & 0.46 & 0.22 & 0.43 & 0.35 & 0.42 & 0.61 & 1.58 & 0.6 \\
\hline G17 & Canberra & 0.31 & 0.45 & 0.22 & 0.45 & 0.29 & 0.34 & 0.50 & 0.61 & 0.4 \\
\hline G18 & Carlita & 0.31 & 0.44 & 0.21 & 0.43 & 0.32 & 0.39 & 0.58 & 0.85 & 0.4 \\
\hline G19 & Carrera & 0.27 & 0.40 & 0.18 & 0.40 & 0.28 & 0.34 & 0.53 & 0.56 & 0.4 \\
\hline G20 & Challenger & 0.32 & 0.46 & 0.24 & 0.46 & 0.32 & 0.37 & 0.55 & 0.54 & 0.4 \\
\hline G21 & Charlotte & 0.32 & 0.45 & 0.24 & 0.45 & 0.36 & 0.42 & 0.63 & 0.61 & 0.4 \\
\hline G22 & Cleopatra & 0.25 & 0.40 & 0.16 & 0.39 & 0.20 & 0.25 & 0.39 & 0.71 & 0.4 \\
\hline G23 & Colomba & 0.33 & 0.46 & 0.24 & 0.46 & 0.35 & 0.41 & 0.61 & 0.70 & 0.4 \\
\hline G24 & Compass & 0.35 & 0.49 & 0.27 & 0.49 & 0.37 & 0.43 & 0.62 & 0.67 & 0.5 \\
\hline G25 & Courage & 0.28 & 0.43 & 0.20 & 0.43 & 0.26 & 0.30 & 0.46 & 0.57 & 0.4 \\
\hline G26 & Crisp4all & 0.25 & 0.35 & 0.17 & 0.36 & 0.37 & 0.45 & 0.73 & 0.72 & 0.4 \\
\hline G27 & Desiree & 0.37 & 0.49 & 0.25 & 0.47 & 0.37 & 0.45 & 0.63 & 1.56 & 0.6 \\
\hline G28 & Evora & 0.35 & 0.48 & 0.28 & 0.48 & 0.41 & 0.47 & 0.68 & 0.53 & 0.5 \\
\hline G29 & Fabula & 0.43 & 0.61 & 0.32 & 0.59 & 0.26 & 0.28 & 0.32 & 0.83 & 0.5 \\
\hline G30 & Faluka & 0.40 & 0.52 & 0.30 & 0.50 & 0.44 & 0.51 & 0.73 & 1.14 & 0.6 \\
\hline G31 & Felsina & 0.31 & 0.43 & 0.22 & 0.42 & 0.35 & 0.41 & 0.63 & 0.84 & 0.5 \\
\hline G32 & Flamenco & 0.33 & 0.46 & 0.22 & 0.45 & 0.30 & 0.36 & 0.51 & 1.10 & 0.5 \\
\hline G33 & Frisia & 0.22 & 0.31 & 0.13 & 0.31 & 0.36 & 0.45 & 0.74 & 0.80 & 0.4 \\
\hline G34 & Hansa & 0.33 & 0.41 & 0.24 & 0.41 & 0.50 & 0.60 & 0.92 & 1.04 & 0.6 \\
\hline G35 & Hermes & 0.29 & 0.34 & 0.20 & 0.35 & 0.55 & 0.67 & 1.06 & 1.20 & 0.6 \\
\hline G36 & Innovator & 0.24 & 0.35 & 0.13 & 0.34 & 0.29 & 0.36 & 0.58 & 1.00 & 0.4 \\
\hline G37 & Ivory Russet & 0.20 & 0.31 & 0.10 & 0.30 & 0.28 & 0.35 & 0.60 & 0.86 & 0.4 \\
\hline G38 & Jaerla & 0.34 & 0.48 & 0.25 & 0.48 & 0.33 & 0.38 & 0.55 & 0.77 & 0.5 \\
\hline G39 & Jazzy & 0.30 & 0.42 & 0.20 & 0.41 & 0.34 & 0.41 & 0.62 & 0.95 & 0.5 \\
\hline G40 & Kastelli & 0.36 & 0.48 & 0.26 & 0.47 & 0.41 & 0.48 & 0.70 & 1.04 & 0.5 \\
\hline
\end{tabular}




\begin{tabular}{|c|c|c|c|c|c|c|c|c|c|c|}
\hline Code & Cultivar & E1 & E2 & E3 & E4 & E5 & E6 & E7 & E8 & Mean \\
\hline G41 & Kennebec & 0.35 & 0.46 & 0.25 & 0.45 & 0.43 & 0.51 & 0.76 & 1.00 & 0.5 \\
\hline G42 & Kondor & 0.34 & 0.44 & 0.25 & 0.44 & 0.44 & 0.52 & 0.78 & 0.86 & 0.5 \\
\hline G43 & Kuras & 0.33 & 0.47 & 0.24 & 0.46 & 0.32 & 0.38 & 0.54 & 0.82 & 0.5 \\
\hline G44 & Kuroda & 0.34 & 0.47 & 0.25 & 0.46 & 0.37 & 0.43 & 0.63 & 0.88 & 0.5 \\
\hline G45 & Lady Christl & 0.24 & 0.34 & 0.16 & 0.34 & 0.39 & 0.48 & 0.77 & 0.80 & 0.4 \\
\hline G46 & Lady Claire & 0.24 & 0.34 & 0.16 & 0.35 & 0.38 & 0.46 & 0.76 & 0.68 & 0.4 \\
\hline G47 & Lady Rosetta & 0.28 & 0.40 & 0.17 & 0.38 & 0.31 & 0.38 & 0.59 & 1.13 & 0.5 \\
\hline G48 & Leonardo & 0.32 & 0.46 & 0.22 & 0.45 & 0.28 & 0.33 & 0.48 & 0.90 & 0.4 \\
\hline G49 & Liseta & 0.31 & 0.46 & 0.22 & 0.45 & 0.29 & 0.34 & 0.49 & 0.60 & 0.4 \\
\hline G50 & Lucinda & 0.36 & 0.50 & 0.26 & 0.49 & 0.32 & 0.37 & 0.51 & 0.92 & 0.5 \\
\hline G51 & Marabel & 0.28 & 0.39 & 0.17 & 0.38 & 0.31 & 0.38 & 0.59 & 1.16 & 0.5 \\
\hline G52 & Marfona & 0.28 & 0.39 & 0.18 & 0.38 & 0.33 & 0.40 & 0.62 & 1.07 & 0.5 \\
\hline G53 & Marilyn & 0.35 & 0.46 & 0.23 & 0.44 & 0.38 & 0.45 & 0.66 & 1.46 & 0.6 \\
\hline G54 & Maris Piper & 0.28 & 0.37 & 0.17 & 0.36 & 0.36 & 0.44 & 0.70 & 1.32 & 0.5 \\
\hline G55 & Markies & 0.30 & 0.41 & 0.22 & 0.41 & 0.43 & 0.51 & 0.80 & 0.76 & 0.5 \\
\hline G56 & Melody & 0.28 & 0.37 & 0.19 & 0.37 & 0.42 & 0.51 & 0.81 & 0.87 & 0.5 \\
\hline G57 & Memphis & 0.39 & 0.52 & 0.29 & 0.51 & 0.40 & 0.46 & 0.64 & 1.02 & 0.5 \\
\hline G58 & Monalisa & 0.30 & 0.43 & 0.21 & 0.42 & 0.34 & 0.40 & 0.61 & 0.80 & 0.4 \\
\hline G59 & Mondial & 0.46 & 0.62 & 0.36 & 0.61 & 0.38 & 0.42 & 0.53 & 0.80 & 0.5 \\
\hline G60 & Mozart & 0.36 & 0.47 & 0.27 & 0.47 & 0.45 & 0.53 & 0.78 & 0.76 & 0.5 \\
\hline G62 & Nicola & 0.31 & 0.36 & 0.22 & 0.37 & 0.57 & 0.68 & 1.08 & 1.12 & 0.6 \\
\hline G63 & Orchestra & 0.34 & 0.46 & 0.25 & 0.46 & 0.39 & 0.46 & 0.68 & 0.76 & 0.5 \\
\hline G64 & Panther & 0.29 & 0.41 & 0.20 & 0.41 & 0.34 & 0.41 & 0.63 & 0.74 & 0.4 \\
\hline G65 & Picasso & 0.42 & 0.54 & 0.33 & 0.54 & 0.48 & 0.55 & 0.78 & 0.87 & 0.6 \\
\hline G66 & Red Scarlett & 0.33 & 0.47 & 0.23 & 0.46 & 0.32 & 0.37 & 0.54 & 0.84 & 0.4 \\
\hline G67 & Rodeo & 0.34 & 0.48 & 0.24 & 0.46 & 0.33 & 0.39 & 0.56 & 1.07 & 0.5 \\
\hline G68 & Ronaldo & 0.38 & 0.52 & 0.29 & 0.51 & 0.35 & 0.41 & 0.56 & 0.84 & 0.5 \\
\hline G69 & Sagitta & 0.31 & 0.46 & 0.22 & 0.45 & 0.29 & 0.34 & 0.49 & 0.60 & 0.4 \\
\hline G70 & Sifra & 0.34 & 0.48 & 0.26 & 0.48 & 0.34 & 0.39 & 0.57 & 0.67 & 0.4 \\
\hline G71 & Spunta & 0.35 & 0.48 & 0.26 & 0.47 & 0.40 & 0.46 & 0.67 & 0.91 & 0.5 \\
\hline G72 & Sylvana & 0.30 & 0.40 & 0.20 & 0.39 & 0.38 & 0.46 & 0.71 & 1.00 & 0.5 \\
\hline G73 & Taurus & 0.28 & 0.41 & 0.19 & 0.40 & 0.29 & 0.35 & 0.54 & 0.78 & 0.4 \\
\hline G74 & Timate & 0.32 & 0.44 & 0.23 & 0.43 & 0.39 & 0.46 & 0.70 & 0.92 & 0.5 \\
\hline G75 & Triplo & 0.29 & 0.43 & 0.20 & 0.42 & 0.30 & 0.35 & 0.53 & 0.82 & 0.4 \\
\hline G76 & Victoria & 0.29 & 0.40 & 0.19 & 0.39 & 0.35 & 0.42 & 0.65 & 0.99 & 0.5 \\
\hline G77 & Vivaldi & 0.31 & 0.44 & 0.23 & 0.44 & 0.35 & 0.41 & 0.61 & 0.62 & 0.4 \\
\hline G78 & Volumia & 0.35 & 0.50 & 0.25 & 0.49 & 0.29 & 0.34 & 0.47 & 0.91 & 0.5 \\
\hline G79 & Voyager & 0.36 & 0.48 & 0.26 & 0.47 & 0.40 & 0.46 & 0.67 & 0.93 & 0.5 \\
\hline G80 & VR 808 & 0.20 & 0.27 & 0.11 & 0.27 & 0.40 & 0.50 & 0.84 & 1.18 & 0.5 \\
\hline G81 & Zina Red & 0.33 & 0.42 & 0.23 & 0.42 & 0.44 & 0.53 & 0.80 & 1.00 & 0.5 \\
\hline
\end{tabular}

Mean $\quad 0.31 \quad 0.44 \quad 0.22 \quad 0.43 \quad 0.35$ 0.42

$0.63 \quad 0.89$

Code $=$ Genotype code, E1= Debre-Tabor low N Rainfed, E2= Debre-Tabor high N Rainfed, E3= Injbara low N Rainfed, E4=Injbara high N

Rainfed, E5=Injbara low N irrigation, E6= Injbara high $\mathrm{N}$ irrigation, E7= Koga low N irrigation, E8=Koga high N irrigation 
Supplementary Table 3. Mean NUE (kg/kg) of 81 potato cultivars (G1to G81) tested across the test environments within the two mega environments, rainfed (E1 to E4) and irrigation mega environment (E5 to E8)

\begin{tabular}{|c|c|c|c|c|c|c|c|c|c|c|c|}
\hline \multirow[b]{2}{*}{ Code } & \multirow[b]{2}{*}{ Cultivars } & \multicolumn{9}{|c|}{ Rainfed mega environment Irrigation mega environment } & \multirow[b]{2}{*}{ mean } \\
\hline & & E1 & E2 & E3 & $\mathrm{E} 4$ & mean & E5 & E6 & E7 & E8 & \\
\hline G1 & Adora & 43.63 & 8.66 & 38.75 & 21.18 & 28.06 & 21.12 & 4.88 & 56.73 & 47.11 & 32.5 \\
\hline G2 & Agata & 29.7 & 8.52 & 23.07 & 10.72 & 18.00 & 18.42 & 12.13 & 143.26 & 52.94 & 56.7 \\
\hline G3 & Agria & 28.36 & 11.72 & 40.95 & 15.71 & 24.19 & 84.23 & 31.18 & 165.45 & 40.13 & 80.3 \\
\hline G4 & Almera & 23.3 & 8.73 & 35.85 & 14.27 & 20.54 & 35.74 & 16.15 & 141.23 & 75.49 & 67.2 \\
\hline G5 & Ambition & 43.12 & 17.68 & 44.34 & 15.39 & 30.13 & 39.14 & 15.32 & 91.52 & 53.65 & 49.9 \\
\hline G6 & Annabelle & 23.31 & 8.16 & 31.69 & 15.35 & 19.63 & 29.8 & 13.68 & 82.57 & 31.91 & 39.5 \\
\hline G7 & Arinda & 58.64 & 18.41 & 38.6 & 19.66 & 33.83 & 35.34 & 12.58 & 134.94 & 46.14 & 57.3 \\
\hline G8 & Arizona & 28.35 & 16.01 & 35.92 & 12.7 & 23.25 & 54.8 & 13.31 & 113.86 & 41.83 & 56.0 \\
\hline G9 & Asterix & 57.8 & 27.37 & 68.49 & 22.84 & 44.13 & 20.57 & 9.84 & 86.07 & 35.03 & 37.9 \\
\hline G10 & Bafana & 46.92 & 12.96 & 35.96 & 18.36 & 28.55 & 48.48 & 22.09 & 102.49 & 63.01 & 59.0 \\
\hline G11 & Baraka & 66.23 & 16.62 & 47.05 & 20.97 & 37.72 & 20.1 & 7.27 & 72.65 & 39.56 & 34.9 \\
\hline G12 & Bartina & 31.83 & 13.17 & 45.56 & 14.48 & 26.26 & 15.19 & 9.34 & 55.69 & 38.47 & 29.7 \\
\hline G13 & Bellini & 24.24 & 17.4 & 58.08 & 14.89 & 28.65 & 25.9 & 12.05 & 92.14 & 37.96 & 42.0 \\
\hline G14 & Berber & 20.44 & 8.8 & 36.48 & 11.67 & 19.35 & 15.21 & 7.58 & 79.87 & 66.8 & 42.4 \\
\hline G15 & Bintje & 41.07 & 16.46 & 39.74 & 22.32 & 29.90 & 39.17 & 11.47 & 113.67 & 63.96 & 57.1 \\
\hline G16 & Caesar & 45.83 & 20.48 & 52.57 & 22.07 & 35.24 & 23.64 & 13.6 & 94.49 & 31.14 & 40.7 \\
\hline G17 & Canberra & 26.09 & 16.21 & 32.97 & 19.14 & 23.60 & 24.04 & 5.05 & 66.29 & 33.97 & 32.3 \\
\hline G18 & Carlita & 31.32 & 11.58 & 69.57 & 16.71 & 32.30 & 28.29 & 7.87 & 65.4 & 66.8 & 42.1 \\
\hline G19 & Carrera & 17.03 & 10.17 & 34.5 & 16.18 & 19.47 & 35.53 & 12.7 & 69.78 & 34.95 & 38.2 \\
\hline $\mathrm{G} 20$ & Challenger & 43.13 & 14.45 & 47.49 & 24.22 & 32.32 & 31.91 & 12.41 & 82.25 & 44.77 & 42.8 \\
\hline $\mathrm{G} 21$ & Charlotte & 41.42 & 13.42 & 54.81 & 19.65 & 32.33 & 52.71 & 20.43 & 127.55 & 38.05 & 59.7 \\
\hline G22 & Cleopatra & 32.93 & 10.69 & 40.61 & 21.93 & 26.54 & 33.86 & 7.54 & 60.47 & 23.23 & 31.3 \\
\hline $\mathrm{G} 23$ & Colomba & 26.3 & 10.72 & 50.93 & 16.73 & 26.17 & 17.87 & 10.79 & 164.95 & 49.35 & 60.7 \\
\hline $\mathrm{G} 24$ & Compass & 54.61 & 18.84 & 46.32 & 23.41 & 35.80 & 38.9 & 10.77 & 97.84 & 41.56 & 47.3 \\
\hline G25 & Courage & 44.41 & 20.65 & 55.15 & 25.06 & 36.32 & 29.44 & 9 & 94.58 & 29.66 & 40.7 \\
\hline G26 & Crisp4all & 40.21 & 13.07 & 30.14 & 22.57 & 26.50 & 32.42 & 13.64 & 97.13 & 41.32 & 46.1 \\
\hline $\mathrm{G} 27$ & Desiree & 45.51 & 23.34 & 53.45 & 22.02 & 36.08 & 29.23 & 13.24 & 112.19 & 48.95 & 50.9 \\
\hline G28 & Evora & 26.96 & 13.94 & 41.76 & 18.04 & 25.18 & 22.28 & 8.75 & 111.38 & 39.63 & 45.5 \\
\hline G29 & Fabula & 31.94 & 15.09 & 39.76 & 21.48 & 27.07 & 26.96 & 9.25 & 61.45 & 57.84 & 38.9 \\
\hline G30 & Faluka & 29.76 & 10.33 & 28.1 & 18.53 & 21.68 & 51.22 & 16.76 & 123.5 & 90.23 & 70.4 \\
\hline G31 & Felsina & 32.58 & 14.6 & 48.25 & 25.76 & 30.30 & 58.84 & 19.12 & 100.04 & 48.15 & 56.5 \\
\hline G32 & Flamenco & 25.62 & 12.82 & 42.29 & 18.58 & 24.83 & 31.89 & 16.79 & 50.37 & 29.9 & 32.2 \\
\hline G33 & Frisia & 35.01 & 9.63 & 46.97 & 21.65 & 28.32 & 33.48 & 6.52 & 100.75 & 54.79 & 48.9 \\
\hline G34 & Hansa & 32.76 & 24.62 & 36.23 & 21.3 & 28.73 & 45.34 & 28.77 & 109.82 & 63.56 & 61.9 \\
\hline G35 & Hermes & 42.97 & 16.21 & 43.1 & 16.11 & 29.60 & 53.76 & 25.33 & 145.12 & 75.19 & 74.9 \\
\hline G36 & Innovator & 32.53 & 9.28 & 46.61 & 23.05 & 27.87 & 30.29 & 10.87 & 87.69 & 52.1 & 45.2 \\
\hline G37 & Ivory Russet & 32.93 & 10.9 & 20.24 & 14.24 & 19.58 & 46.61 & 16.52 & 114.75 & 50.72 & 57.2 \\
\hline G38 & Jaerla & 45.04 & 13.95 & 37.94 & 17.59 & 28.63 & 74.14 & 22.99 & 71.02 & 48.1 & 54.1 \\
\hline G39 & Jazzy & 49.72 & 13.94 & 21.16 & 15.75 & 25.14 & 64.19 & 24.12 & 116.31 & 63.38 & 67.0 \\
\hline
\end{tabular}




\begin{tabular}{|c|c|c|c|c|c|c|c|c|c|c|c|}
\hline \multirow[b]{2}{*}{ Code } & \multirow[b]{2}{*}{ Cultivars } & \multicolumn{9}{|c|}{ Rainfed mega environment Irrigation mega environment } & \multirow[b]{2}{*}{ mean } \\
\hline & & E1 & E2 & E3 & E4 & mean & E5 & E6 & E7 & E8 & \\
\hline G40 & Kastelli & 34.94 & 12.44 & 44.59 & 21.85 & 28.46 & 43.77 & 18.21 & 140.23 & 71.4 & 68.4 \\
\hline G41 & Kennebec & 31.64 & 18.92 & 41.38 & 25.88 & 29.46 & 55.6 & 16.37 & 88.91 & 64.98 & 56.5 \\
\hline G42 & Kondor & 38.93 & 15.52 & 32.3 & 19.34 & 26.52 & 56.22 & 16.9 & 96.67 & 62.13 & 58.0 \\
\hline G43 & Kuras & 70.17 & 28.27 & 67.02 & 33.31 & 49.69 & 40.58 & 18.19 & 66.74 & 48.31 & 43.5 \\
\hline G44 & Kuroda & 38.97 & 18.28 & 41.93 & 20.52 & 29.93 & 48.76 & 19.4 & 140.5 & 58.61 & 66.8 \\
\hline G45 & Lady Christl & 18.68 & 16.18 & 39.19 & 13.16 & 21.80 & 34.12 & 16.87 & 144.89 & 45.65 & 60.4 \\
\hline G46 & Lady Claire & 43.13 & 15.25 & 47.07 & 24.81 & 32.57 & 53.93 & 18.79 & 139.39 & 44.15 & 64.1 \\
\hline G47 & Lady Rosetta & 56.37 & 24.91 & 37.2 & 23.22 & 35.43 & 46.95 & 13.12 & 140.48 & 71.29 & 68.0 \\
\hline G48 & Leonardo & 39.81 & 10.79 & 43.49 & 17.09 & 27.80 & 38.33 & 13.41 & 94.65 & 47.65 & 48.5 \\
\hline G49 & Liseta & 26.42 & 17.19 & 37.2 & 16.08 & 24.22 & 37.34 & 12.02 & 54.63 & 36.83 & 35.2 \\
\hline G50 & Lucinda & 22.06 & 10.77 & 32.34 & 16.45 & 20.41 & 54.39 & 16.09 & 73.12 & 32.73 & 44.1 \\
\hline G51 & Marabel & 39.24 & 10.5 & 22.6 & 15.78 & 22.03 & 19.53 & 6.83 & 119.65 & 70.76 & 54.2 \\
\hline G52 & Marfona & 27.27 & 9 & 26.68 & 11.22 & 18.54 & 48.7 & 27.99 & 113.48 & 45.62 & 59.0 \\
\hline G53 & Marilyn & 34.62 & 18.87 & 51.25 & 19.79 & 31.13 & 40.97 & 14.87 & 138.03 & 89.02 & 70.7 \\
\hline G54 & Maris Piper & 54.77 & 13.33 & 34.39 & 12.26 & 28.69 & 38.1 & 13.46 & 98.18 & 57.61 & 51.8 \\
\hline G55 & Markies & 53.28 & 14.32 & 45.98 & 20.18 & 33.44 & 53.54 & 24.24 & 124.18 & 51.05 & 63.3 \\
\hline G56 & Melody & 27.95 & 14.06 & 30.66 & 21.99 & 23.67 & 43.27 & 19.03 & 116.59 & 54.19 & 58.3 \\
\hline G57 & Memphis & 21.76 & 14.16 & 32.93 & 16.6 & 21.36 & 54.66 & 17.87 & 124.4 & 73.02 & 67.5 \\
\hline G58 & Monalisa & 34.46 & 9.71 & 26.29 & 15.29 & 21.44 & 47.37 & 20.92 & 96.81 & 57.59 & 55.7 \\
\hline G59 & Mondial & 54.59 & 18.9 & 52.09 & 23.28 & 37.22 & 69.86 & 14.67 & 84.7 & 43.68 & 53.2 \\
\hline G60 & Mozart & 29.59 & 15.02 & 31.53 & 18.3 & 23.61 & 52.59 & 10.35 & 105.07 & 46.35 & 53.6 \\
\hline G61 & Navigator & 39.74 & 16.07 & 48.85 & 20.46 & 31.28 & 49.6 & 16.54 & 92.2 & 34.48 & 48.2 \\
\hline G62 & Nicola & 44.43 & 13.72 & 43.84 & 20.74 & 30.68 & 76.82 & 26.54 & 114.91 & 35.07 & 63.3 \\
\hline G63 & Orchestra & 25.67 & 9.69 & 35.72 & 25.56 & 24.16 & 76.29 & 23.83 & 102.9 & 43.13 & 61.5 \\
\hline G64 & Panther & 30.2 & 9.59 & 34.07 & 16.91 & 22.69 & 36.99 & 15.71 & 104.18 & 51.41 & 52.1 \\
\hline G65 & Picasso & 40.66 & 19.6 & 38.65 & 23.35 & 30.57 & 39.02 & 21.19 & 123.14 & 48.67 & 58.0 \\
\hline G66 & Red Scarlett & 24.9 & 11.67 & 32.42 & 18.62 & 21.90 & 40.29 & 16.72 & 116.38 & 71.05 & 61.1 \\
\hline G67 & Rodeo & 30.25 & 16.58 & 42.97 & 30.24 & 30.01 & 32.75 & 15.04 & 66.14 & 40.76 & 38.7 \\
\hline G68 & Ronaldo & 26.97 & 13.62 & 20.36 & 18.21 & 19.79 & 28.59 & 12.65 & 110.22 & 66.28 & 54.4 \\
\hline G69 & Sagitta & 35.36 & 17.34 & 55.49 & 19.15 & 31.84 & 36.46 & 10.22 & 63.17 & 37.8 & 36.9 \\
\hline G70 & Sifra & 34.6 & 14.4 & 42.47 & 12.88 & 26.09 & 43.23 & 18.5 & 82.44 & 47.66 & 48.0 \\
\hline G71 & Spunta & 32.49 & 19.22 & 35.75 & 20.52 & 27.00 & 44.77 & 26.2 & 84.89 & 76.9 & 58.2 \\
\hline G72 & Sylvana & 32.05 & 10.61 & 31.81 & 16.65 & 22.78 & 57.39 & 12.03 & 134.21 & 72.01 & 68.9 \\
\hline G73 & Taurus & 46.57 & 16.17 & 48.91 & 19.12 & 32.69 & 37.48 & 17.03 & 93.89 & 43.26 & 47.9 \\
\hline G74 & Timate & 43.96 & 17.36 & 51.56 & 17.54 & 32.61 & 45.19 & 16.34 & 117.91 & 53.23 & 58.2 \\
\hline G75 & Triplo & 30.8 & 9.76 & 44.97 & 21.59 & 26.78 & 47.07 & 15.25 & 70.01 & 46.55 & 44.7 \\
\hline G76 & Victoria & 37.06 & 11.44 & 30.88 & 18.24 & 24.41 & 51 & 19.55 & 102.02 & 77.44 & 62.5 \\
\hline G77 & Vivaldi & 34.39 & 12.22 & 38.42 & 14.65 & 24.92 & 30.75 & 19.57 & 70.29 & 36.34 & 39.2 \\
\hline G78 & Volumia & 40.41 & 7.42 & 37.5 & 17.23 & 25.64 & 38.96 & 19.58 & 76.69 & 40.29 & 43.9 \\
\hline G79 & Voyager & 53.57 & 18.01 & 38.96 & 17.49 & 32.01 & 51.95 & 18.46 & 105.58 & 61.67 & 59.4 \\
\hline G80 & VR 808 & 36.05 & 14.69 & 41.12 & 22.98 & 28.71 & 27.96 & 14.78 & 100.43 & 48.44 & 47.9 \\
\hline G81 & Zina Red & 49.64 & 17.34 & 41.49 & 21.93 & 32.60 & 47.13 & 19.14 & 116.36 & 43.55 & 56.6 \\
\hline Mean & & 37.06 & 14.60 & 40.69 & 19.17 & 27.88 & 41.21 & 15.63 & 101.32 & 51.11 & 52.3 \\
\hline
\end{tabular}

Code = genotype code, E1= Debre-Tabor low N Rainfed, E2= Debre-Tabor high N Rainfed, E3= Injbara low N Rainfed, E4=Injbara high N

Rainfed, E5=Injbara low N irrigation, E6= Injbara high N irrigation, E7= Koga low N irrigation, E8=Koga high N irrigation 
Supplementary Table 4. Numerical values for the genotypes based on the mean vs instability view of the GGE biplot for NUE of potato cultivars in rainfed mega environment (Figure 5).

\begin{tabular}{|c|c|c|c|c|c|}
\hline Code & Genotypes & Mean & Instability & $\begin{array}{l}\text { Rank on closeness } \\
\text { to ideal genotype }\end{array}$ & $\begin{array}{l}\text { Distance to } \\
\text { ideal genotype }\end{array}$ \\
\hline G43 & Kuras & 3.022 & 0.066 & 1 & 1.1 \\
\hline G9 & Asterix & 2.1 & -0.047 & 2 & 1.8 \\
\hline G25 & Courage & 1.219 & -0.206 & 3 & 2.5 \\
\hline G27 & Desiree & 1.172 & -0.031 & 4 & 2.5 \\
\hline G59 & Mondial & 1.169 & 0.127 & 5 & 2.5 \\
\hline G47 & Lady Rosetta & 1.178 & 0.612 & 6 & 2.6 \\
\hline G24 & Compass & 1.031 & 0.26 & 7 & 2.6 \\
\hline G16 & Caesar & 0.999 & -0.046 & 8 & 2.7 \\
\hline G11 & Baraka & 1.04 & 0.545 & 9 & 2.7 \\
\hline G46 & Lady Claire & 0.67 & -0.12 & 10 & 2.9 \\
\hline G67 & Rodeo & 0.677 & -0.437 & 11 & 2.9 \\
\hline G7 & Arinda & 0.683 & 0.617 & 12 & 2.9 \\
\hline G81 & Zina Red & 0.625 & 0.264 & 13 & 2.9 \\
\hline G65 & Picasso & 0.565 & 0.116 & 14 & 3 \\
\hline G20 & Challenger & 0.6 & -0.129 & 15 & 3 \\
\hline G34 & Hansa & 0.488 & 0.103 & 16 & 3 \\
\hline G41 & Kennebec & 0.541 & -0.233 & 17 & 3 \\
\hline G55 & Markies & 0.543 & 0.24 & 18 & 3 \\
\hline G73 & Taurus & 0.505 & 0.06 & 19 & 3 \\
\hline G74 & Timate & 0.484 & -0.013 & 20 & 3.1 \\
\hline G53 & Marilyn & 0.485 & -0.259 & 21 & 3.1 \\
\hline G69 & Sagitta & 0.481 & -0.35 & 22 & 3.1 \\
\hline G61 & Navigator & 0.423 & -0.135 & 23 & 3.1 \\
\hline G31 & Felsina & 0.48 & -0.434 & 24 & 3.1 \\
\hline G79 & Voyage & 0.424 & 0.528 & 25 & 3.1 \\
\hline G44 & Kuroda & 0.363 & 0.041 & 26 & 3.1 \\
\hline G15 & Bintje & 0.361 & 0.077 & 27 & 3.1 \\
\hline G21 & Charlotte & 0.41 & -0.257 & 28 & 3.1 \\
\hline G62 & Nicola & 0.289 & 0.056 & 29 & 3.2 \\
\hline G80 & VR 808 & 0.221 & -0.118 & 30 & 3.2 \\
\hline G5 & Ambition & 0.176 & 0.191 & 31 & 3.3 \\
\hline G71 & Spunta & 0.117 & 0.045 & 32 & 3.3 \\
\hline G35 & Hermes & 0.101 & 0.178 & 33 & 3.3 \\
\hline G18 & Carlita & 0.265 & -0.811 & 34 & 3.4 \\
\hline G40 & Kastelli & 0.087 & -0.236 & 35 & 3.4 \\
\hline G29 & Fabula & 0.027 & -0.146 & 36 & 3.4 \\
\hline G13 & Bellini & 0.041 & -0.584 & 37 & 3.4 \\
\hline G38 & Jaerla & -0.018 & 0.284 & 38 & 3.4 \\
\hline G10 & Bafana & -0.035 & 0.344 & 39 & 3.5 \\
\hline G33 & Frisia & -0.024 & -0.327 & 40 & 3.5 \\
\hline G26 & Crisp4all & -0.072 & 0.227 & 41 & 3.5 \\
\hline G36 & Innovator & -0.025 & -0.415 & 42 & 3.5 \\
\hline
\end{tabular}




\begin{tabular}{|c|c|c|c|c|c|}
\hline Code & Genotypes & Mean & Instability & $\begin{array}{l}\text { Rank on closeness } \\
\text { to ideal genotype }\end{array}$ & $\begin{array}{l}\text { Distance to } \\
\text { ideal genotype }\end{array}$ \\
\hline G42 & Kondor & -0.102 & 0.253 & 43 & 3.5 \\
\hline G1 & Adora & -0.115 & 0.071 & 44 & 3.5 \\
\hline G22 & Cleopatra & -0.152 & -0.218 & 45 & 3.6 \\
\hline G75 & Triplo & -0.165 & -0.379 & 46 & 3.6 \\
\hline G48 & Leonardo & -0.209 & -0.01 & 47 & 3.6 \\
\hline G56 & Melody & -0.316 & -0.054 & 48 & 3.6 \\
\hline G28 & Evora & -0.307 & -0.254 & 49 & 3.7 \\
\hline G17 & Canberra & -0.35 & -0.058 & 50 & 3.7 \\
\hline G63 & Orchestra & -0.276 & -0.374 & 51 & 3.7 \\
\hline G54 & Maris Piper & -0.24 & 0.713 & 52 & 3.7 \\
\hline G49 & Liseta & -0.366 & -0.067 & 53 & 3.7 \\
\hline G12 & Bartina & -0.36 & -0.158 & 54 & 3.7 \\
\hline G32 & Flamenco & -0.356 & -0.328 & 55 & 3.7 \\
\hline G70 & Sifra & -0.4 & 0.036 & 56 & 3.7 \\
\hline G60 & Mozart & -0.424 & 0.061 & 57 & 3.7 \\
\hline G23 & Colomba & -0.357 & -0.506 & 58 & 3.7 \\
\hline G76 & Victoria & -0.477 & 0.206 & 59 & 3.8 \\
\hline G77 & Vivaldi & -0.522 & 0.055 & 60 & 3.8 \\
\hline G39 & Jazzy & -0.438 & 0.834 & 61 & 3.8 \\
\hline G78 & Volumia & -0.526 & 0.093 & 62 & 3.8 \\
\hline G3 & Agria & -0.561 & -0.182 & 63 & 3.8 \\
\hline G8 & Arizona & -0.623 & 0.068 & 64 & 3.9 \\
\hline G66 & Red Scarlett & -0.679 & -0.13 & 65 & 3.9 \\
\hline G57 & Memphis & -0.717 & -0.136 & 66 & 3.9 \\
\hline G45 & Lady Christl & -0.724 & -0.251 & 67 & 4 \\
\hline G72 & Sylvana & -0.71 & 0.086 & 68 & 4 \\
\hline G64 & Panther & -0.738 & -0.034 & 69 & 4 \\
\hline G30 & Faluka & -0.756 & 0.072 & 70 & 4 \\
\hline G68 & Ronaldo & -0.844 & 0.243 & 71 & 4 \\
\hline G51 & Marabel & -0.833 & 0.495 & 72 & 4.1 \\
\hline G50 & Lucinda & -0.926 & -0.162 & 73 & 4.1 \\
\hline G58 & Monalisa & -0.924 & 0.291 & 74 & 4.1 \\
\hline G19 & Carrera & -1.037 & -0.337 & 75 & 4.2 \\
\hline G4 & Almera & -1.057 & -0.196 & 76 & 4.2 \\
\hline G37 & Ivory Russet & -1.103 & 0.438 & 77 & 4.2 \\
\hline G6 & Annabelle & -1.127 & -0.13 & 78 & 4.3 \\
\hline G14 & Berber & -1.258 & -0.221 & 79 & 4.4 \\
\hline G52 & Marfona & -1.361 & 0.189 & 80 & 4.4 \\
\hline G2 & Agata & -1.452 & 0.337 & 81 & 4.5 \\
\hline
\end{tabular}

Code $=$ genotype code 
Supplementary table 5. Numerical values for the genotypes based on the mean vs instability view of the GGE biplot for NUE of potato cultivars in rainfed mega environment in Figure 6.

\begin{tabular}{|c|c|c|c|c|c|}
\hline Code & Genotypes & Mean & Instability & $\begin{array}{l}\text { Rank on closeness } \\
\text { to ideal genotype }\end{array}$ & $\begin{array}{l}\text { Distance to ideal } \\
\text { genotype }\end{array}$ \\
\hline G35 & Hermes & 1.465 & 0.16 & 1 & 1.6 \\
\hline G3 & Agria & 1.824 & -1.013 & 2 & 1.6 \\
\hline G39 & Jazzy & 1.118 & -0.324 & 3 & 1.9 \\
\hline G30 & Faluka & 1.103 & 0.646 & 4 & 2 \\
\hline G34 & Hansa & 0.945 & -0.239 & 5 & 2 \\
\hline G57 & Memphis & 0.921 & 0.25 & 6 & 2.1 \\
\hline G40 & Kastelli & 0.87 & 0.456 & 7 & 2.1 \\
\hline G44 & Kuroda & 0.788 & 0.111 & 8 & 2.1 \\
\hline G71 & Spunta & 0.822 & -0.037 & 9 & 2.2 \\
\hline G55 & Markies & 0.801 & -0.352 & 10 & 2.2 \\
\hline G76 & Victoria & 0.802 & 0.203 & 11 & 2.2 \\
\hline G53 & Marilyn & 0.959 & 0.919 & 12 & 2.2 \\
\hline G72 & Sylvana & 0.783 & 0.445 & 13 & 2.2 \\
\hline G47 & Lady Rosetta & 0.699 & 0.583 & 14 & 2.3 \\
\hline G52 & Marfona & 0.691 & -0.567 & 15 & 2.3 \\
\hline G4 & Almera & 0.722 & 0.724 & 16 & 2.3 \\
\hline G10 & Bafana & 0.626 & -0.106 & 17 & 2.3 \\
\hline G46 & Lady Claire & 0.588 & -0.213 & 18 & 2.3 \\
\hline G62 & Nicola & 0.936 & -1.113 & 19 & 2.3 \\
\hline G63 & Orchestra & 0.836 & -0.932 & 20 & 2.3 \\
\hline G79 & Voyager & 0.53 & -0.04 & 21 & 2.4 \\
\hline G66 & Red Scarlett & 0.517 & 0.421 & 22 & 2.4 \\
\hline G42 & Kondor & 0.459 & -0.089 & 23 & 2.4 \\
\hline G21 & Charlotte & 0.425 & -0.426 & 24 & 2.5 \\
\hline G65 & Picasso & 0.368 & -0.083 & 25 & 2.5 \\
\hline G56 & Melody & 0.38 & -0.007 & 26 & 2.5 \\
\hline G58 & Monalisa & 0.409 & -0.179 & 27 & 2.5 \\
\hline G41 & Kennebec & 0.403 & -0.053 & 28 & 2.5 \\
\hline G31 & Felsina & 0.391 & -0.439 & 29 & 2.5 \\
\hline G74 & Timate & 0.29 & 0.047 & 30 & 2.6 \\
\hline G45 & Lady Christl & 0.243 & 0.202 & 31 & 2.6 \\
\hline G38 & Jaerla & 0.553 & -0.957 & 32 & 2.6 \\
\hline
\end{tabular}




\begin{tabular}{|c|c|c|c|c|c|}
\hline Code & Genotypes & Mean & Instability & $\begin{array}{l}\text { Rank on closeness } \\
\text { to ideal genotype }\end{array}$ & $\begin{array}{l}\text { Distance to ideal } \\
\text { genotype }\end{array}$ \\
\hline G81 & Zina Red & 0.264 & -0.262 & 33 & 2.6 \\
\hline G37 & Ivory Russet & 0.249 & -0.043 & 34 & 2.6 \\
\hline G8 & Arizona & 0.084 & -0.22 & 35 & 2.7 \\
\hline G15 & Bintje & 0.118 & 0.474 & 36 & 2.7 \\
\hline G59 & Mondial & 0.163 & -0.614 & 37 & 2.8 \\
\hline G7 & Arinda & -0.009 & 0.285 & 38 & 2.8 \\
\hline G64 & Panther & -0.037 & 0.081 & 39 & 2.8 \\
\hline G68 & Ronaldo & -0.001 & 0.612 & 40 & 2.9 \\
\hline G54 & Maris Piper & -0.065 & 0.221 & 41 & 2.9 \\
\hline G60 & Mozart & -0.087 & -0.052 & 42 & 2.9 \\
\hline G5 & Ambition & -0.096 & 0.034 & 43 & 2.9 \\
\hline G23 & Colomba & -0.052 & 0.826 & 44 & 2.9 \\
\hline G70 & Sifra & -0.074 & -0.294 & 45 & 2.9 \\
\hline $\mathrm{G} 2$ & Agata & -0.122 & 0.718 & 46 & 2.9 \\
\hline G73 & Taurus & -0.207 & -0.176 & 47 & 3 \\
\hline G27 & Desiree & -0.246 & 0.278 & 48 & 3 \\
\hline G61 & Navigator & -0.19 & -0.506 & 49 & 3 \\
\hline G48 & Leonardo & -0.269 & 0.019 & 50 & 3 \\
\hline G80 & VR 808 & -0.318 & 0.171 & 51 & 3.1 \\
\hline G43 & Kuras & -0.267 & -0.319 & 52 & 3.1 \\
\hline G78 & Volumia & -0.28 & -0.434 & 53 & 3.1 \\
\hline G75 & Triplo & -0.286 & -0.327 & 54 & 3.1 \\
\hline G26 & Crisp4all & -0.446 & -0.003 & 55 & 3.2 \\
\hline G24 & Compass & -0.452 & 0.008 & 56 & 3.2 \\
\hline G51 & Marabel & -0.248 & 1.077 & 57 & 3.2 \\
\hline G50 & Lucinda & -0.335 & -0.697 & 58 & 3.2 \\
\hline G33 & Frisia & -0.476 & 0.49 & 59 & 3.2 \\
\hline G36 & Innovator & -0.505 & 0.267 & 60 & 3.2 \\
\hline G77 & Vivaldi & -0.548 & -0.419 & 61 & 3.3 \\
\hline G20 & Challenger & -0.587 & 0.028 & 62 & 3.3 \\
\hline G67 & Rodeo & -0.676 & -0.236 & 63 & 3.4 \\
\hline G18 & Carlita & -0.624 & 0.544 & 64 & 3.4 \\
\hline G13 & Bellini & -0.732 & 0.059 & 65 & 3.4 \\
\hline G28 & Evora & -0.732 & 0.361 & 66 & 3.4 \\
\hline
\end{tabular}




\begin{tabular}{llllll}
\hline Code & Genotypes & Mean & Instability & Rank on closeness & Distance to ideal \\
& & & to ideal genotype & genotype \\
\hline G16 & Caesar & -0.798 & -0.072 & 67 & 3.4 \\
G6 & Evora & -0.787 & -0.217 & 68 & 3.5 \\
G19 & Carrera & -0.798 & -0.282 & 69 & 3.5 \\
G29 & Fabula & -0.778 & 0.332 & 70 & 3.5 \\
G14 & Berber & -0.726 & 0.825 & 71 & 3.5 \\
G25 & Courage & -0.927 & -0.026 & 72 & 3.6 \\
G69 & Sagitta & -0.904 & -0.195 & 73 & 3.6 \\
G49 & Liseta & -0.906 & -0.334 & 74 & 3.6 \\
G9 & Asterix & -1.027 & 0.127 & 75 & 3.6 \\
G32 & Flamenco & -0.947 & -0.567 & 76 & 3.7 \\
G11 & Baraka & -1.195 & 0.23 & 77 & 3.8 \\
G1 & Adora & -1.3 & 0.347 & 78 & 3.9 \\
G22 & Cleopatra & -1.344 & -0.344 & 79 & 3.9 \\
G12 & Bartina & -1.363 & 0.119 & 80 & 4 \\
G17 & Canberra & -1.381 & 0.112 & & \\
\hline Code geta & & & & 7.9 \\
\hline
\end{tabular}

Code $=$ genotype code 


\section{Summary}

Nitrogen use efficiency (NUE) improvement in agricultural crops basically has two main goals; economic gain and N pollution reduction. However, breeding for NUE is not an easy task, as NUE is a complex trait. Dissecting the complexity of such quantitative traits into component loci and identify the genetic factors that influence quantitative traits will significantly increase the success of breeding for NUE.

Potato is the most important non-grain food crop in the world; however it is poor in nutrient use efficiency. As a first step towards understanding the genetic basis for NUE and NUErelated agronomic and physiological traits in potato, we make use of the CxE experimental population and commercial potato (Ethiopian and European) cultivars. Our experimental population was extensively evaluated under low and high Nitrogen conditions at two locations (Debre-Tabor and Injibara) in 2013, and the data collected from these experiments were used for a diversity study. The analysis of variance revealed that, the effect of $\mathrm{N}$ level on most traits was significant at each location. Low $\mathrm{N}$ availability caused a significant $(\mathrm{P} \leq 0.01)$ reduction (23\% in Debre-Tabor and $40 \%$ in Injibara) in potato maximum canopy cover. The area under the canopy curve for the entire crop growth cycle (AUC), representing the total light intercepted by a cultivar during the growth cycle, was significantly $((\mathrm{P} \leq 0.01)$ affected (28\% reduction at Debre-Tabor and $37 \%$ at Injibara) by low N availability. Similarly, the reduction due to $\mathrm{N}$ shortage was significant for tuber yield and yield related traits at both locations. The tuber yield was reduced by $30 \%$ in Debre-Tabor and $48 \%$ in Injibara. Of the two tuber yield components, tuber number was reduced by $16 \%$ in Debre-Tabor and $38 \%$ in Injibara, while average tuber weight was reduced by $17 \%$ in Debre-tabor and $16 \%$ in Injibara. This higher tuber yield and tuber number reduction at Injibara may be related to the low $\mathrm{pH}$ (acidic property) of the soil.

The variation among cultivars was significant for all traits at both locations. In potato, genetic variation of NUE is largely explained by maturity type. Based on the maturity data collected in our experiment, we have classified our cultivars into an early, intermediate and late maturity group. The variation between the late maturity group and the intermediate and early maturity group was visible for most traits (including tuber yield, tuber dry matter $\%$ and area under the canopy curve) under low and high $\mathrm{N}$ conditions in Debre-Tabor. The late maturity group cultivars had higher values of area under the canopy curve as well as tuber yield at both $\mathrm{N}$ levels in Debre-Tabor. In Injibara however, the variation among these maturity groups was 
lower, and even negligible for tuber yield per plant (TYPP) at both $\mathrm{N}$ levels, even the late maturity group had higher values for AUC compared to the early and intermediate maturity group. Most Dutch cultivars were classified in the early and intermediate maturity group while most Ethiopian cultivars clustered in the late maturity group at both locations, suggesting that maturity is the main factor for the variation between the Ethiopian and the Dutch cultivars. Our results suggest irrespective of the locations and $\mathrm{N}$ levels that some of the late maturing potato cultivars, such as Kuras and Asterix from Europe, and most Ethiopian varieties showed relatively a better NUE performance compared to the other European cultivars indicating the persistent inherent potential of the cultivars for NUE at both low and high $\mathrm{N}$ conditions.

For the genetic diversity study, clustering was carried out based on the generalized $\mathrm{D}^{2}$ distances by average linkage method of hierarchical clustering called Unweighted Pair Group Methods with Arithmetic-average (UPGMA). Genetic distance within and between clusters was calculated using the generalized Mahalanobis's $\mathrm{D}^{2}$ statistics. These enable us to visualize genetic relationships of cultivar phenotypes at low and high $\mathrm{N}$ conditions across locations. Subsequently, the 97 cultivars were clustered into 9 and 11 genetically distinct classes at low and high $\mathrm{N}$ respectively. Most of the cultivars grouped in a single cluster (cluster I) at both $\mathrm{N}$ levels; $87 \%$ at low $\mathrm{N}$ and $65 \%$ at high $\mathrm{N}$. The Ethiopian cultivars Ater-Ababa, Awash and Gudenie were included in the largest cluster (cluster-I) at low N level, the rest were Dutch cultivars. Most Dutch cultivars were clustered in cluster-I, while the Ethiopian cultivars were distinctly grouped in cluster-IV and V at low $\mathrm{N}$ and in cluster-IV, VI and VII at high $\mathrm{N}$ level, suggesting the presence of significant genetic distance between the European and the Ethiopian potato cultivars. The highest inter-cluster genetic distance was observed between two single cultivars, Zengena and Orchestra, with a genetic distance of 296.14 at low N, and between cluster- $\mathrm{V}$ and a single cultivar, Agerie with a genetic distance of 520.06 at high $\mathrm{N}$. Days to maturity, plant height, area under the canopy curve, tuber yield and NUE were the traits that contributed most to the difference between the European and the Ethiopian set of cultivars at both $\mathrm{N}$ levels. In general, we found most contrasting cluster means with significant inter cluster genetic distance at both low and high $\mathrm{N}$ level for our targeted traits such as days to maturity, NUE, tuber yield and area under the canopy curve, which indicates the presence of wider genetic variation in our population and suggesting the possibility to use cultivars in different clusters as parents for hybridization at the respective $\mathrm{N}$ levels. 
We classified the observed variation in the potato cultivars into heritable and non-heritable components, and values for broad sense heritability $\left(\mathrm{H}^{2}\right)$, coefficient of phenotypic variation (PCV) and genotypic variation (GCV), and genetic advance as percent of mean (GA\%) obtained under low and high $\mathrm{N}$ level. Heritability varied over treatments and locations between 0.33 and 0.95, as an example NUE has high heritability values in Debre-Tabor at low and high $\mathrm{N}$ levels ( 0.80 and 0.72 respectively), but only 0.4 at both $\mathrm{N}$ levels in Injibara, indicating the contribution of the environment to the total NUE variation was high in Injibara compared to Debre-Tabor. The results revealed that estimates of phenotypic coefficient of variation were quite close to the estimates of genotypic coefficient of variation for most measured traits over treatments at each location, indicating negligible environmental effect on the variance of traits.

We identified the association of agronomic and physiological traits with NUE using person correlation and path coefficient analysis, and important traits that contribute to the indirect selection of $\mathrm{N}$ use efficient cultivars. Consequently, strong phenotypic correlations were detected between NUE and tuber number per plant, days to maturity, tuber dry matter \%, maximum canopy cover (Vmax) and area under the canopy curve (AUC) under both low and high $\mathrm{N}$ conditions. Some traits which have strong correlation with NUE did not have a strong direct effect on NUE, however they had a strong indirect effect via the other traits. As an example, the correlation coefficient value between NUE and area under the canopy curve was high (0.6 and 0.8) under low and high $\mathrm{N}$ condition respectively. However, area under the canopy curve (AUC) had a negligible direct effect on NUE, while its high indirect effect was via tuber dry matter $\%$ and tuber number, counterbalanced the negligible direct effect on the observed variation of NUE. Overall, the path coefficient analysis revealed that, the largest direct contributions to the variation observed in NUE under low and high N condition was via tuber dry matter $\%$ and tuber number. In general we propose that, potato cultivars can be exploited for NUE improvement through improving and pyramiding of component traits at both low and high $\mathrm{N}$ levels.

The C x E bi-parental diploid population was evaluated for NUE in 2014 at Koga under irrigation, and Injibara and Debre-Tabor, under rain fed production systems. The data were used for QTL mapping, and QTL analysis was performed using Interval mapping. Subsequently, Multiple QTL Mapping (MQM) was performed with cofactors selected as the markers nearest to the QTLs detected by interval mapping. For this analysis 534 SNP, SSR and AFLP markers with a total genetic map distance of $1326 \mathrm{cM}$ were employed, equivalent to 
an average distance between markers of $2.5 \mathrm{cM}$, assuming that the markers are evenly distributed.

The NUE evaluation of the $\mathrm{C} x \mathrm{E}$ population at field conditions under rain fed and irrigation production systems comprised traits, such as chlorophyll content, days to maturity, maximum canopy cover (Vmax), area under the canopy curve (AUC), tuber number, tuber yield, and NUE. Significant genetic variation was observed for most traits considered in this study under both low and high $\mathrm{N}$ conditions. The genetic variation and heritability estimates were medium to high for most measured traits under both $\mathrm{N}$ conditions. A total of 52 putative QTLs were identified for the ten traits distributed in 13 QTL regions on seven linkage groups, of which 28 QTLs were detected under low $\mathrm{N}$ while 24 QTLs were detected under high N condition. The QTLs for area under the canopy curve, plant height, maximum canopy cover (Vmax), days to maturity, tuber number, tuber yield and NUE were co-localized on linkage group-V in the 21 to $38 \mathrm{cM}$ region. This co-localization of QTLs of different traits in the same chromosomal region suggests the existence of genetic and functional relationship between traits. Of the identified QTLs in this study, 11 repeatable QTLs that contained 29 individual QTLs were generated under low or high $\mathrm{N}$ specific condition or under both $\mathrm{N}$ conditions, at least over two experimental locations. The occurrence of these $\mathrm{N}$ level and location specific QTLs revealed the presence of QTL $x$ environment interaction (QEI), reflected by the differential expression of genes at different $\mathrm{N}$ environments. Among the QTL regions, chromosome V harboured QTLs for multiple traits under low and or high $\mathrm{N}$ conditions. The region may be enriched with key Nitrogen metabolism genes, however the presence of the CDF1 (or earliness) gene in the region may be the main hurdle to use the QTLs on this linkage group for NUE improvement in potato. To that end uncoupling of the earliness gene with QTLs for NUE improvement would have to be achieved.

Commercial potato cultivars evaluated under rain fed production system in 2013 were once more evaluated under irrigation production system in 2015, and the data were used for association mapping. The association panel was genotyped using SNP array markers. Genome-wide association mapping study (GWAS) was deployed to identify markers associated with NUE and NUE related agronomic and physiological traits under low and high $\mathrm{N}$ environments. The over-location combined analysis of variance revealed that the variation due to $\mathrm{N}$ levels, genotypes and locations were highly significant for almost all of the traits measured. Various genotypes reacted differently to varying locations as indicated by the highly significant $(\mathrm{P} \leq 0.01$ and 0.001$)$ interaction effect of genotype $(\mathrm{G}) \times$ location $(\mathrm{L})$ on most 
physiological and agronomic traits including NUE, while interaction of genotype $(\mathrm{G}) \times \mathrm{N}$ level (N) was not significant for most traits compared to the interaction of Genotype $\mathrm{x}$ Location. The estimates of genotypic variance $\left(\sigma^{2} \mathrm{~g}\right)$ were low compared to estimates of environmental variance $\left(\sigma^{2} \mathrm{e}\right)$ and Genotype $\mathrm{x}$ Environment interaction variance $\left(\sigma^{2} \mathrm{ge}\right)$ for all measured traits in both $\mathrm{N}$ levels, suggesting the presence of high Genotype x Environment interaction. Consequently, due to the noise of the environment most traits had low to medium estimates of broad sense heritability $\left(\mathrm{H}^{2}\right)$ under both $\mathrm{N}$ levels. In particular, the genotype-by-location interaction, which includes production season, had larger contributions to the total genotypeby-environment interaction than the genotype-by-N level interaction, indicating a significant effect of experimental locations as well as production seasons on the performance of potato cultivars.

A total of 77 marker- trait associations (MTAs) were detected under both low and high $\mathrm{N}$ conditions. Different gene models were deployed to identify the marker trait associations, and most of the MTAs were identified using dominant genetic models. Similar as in linkage mapping, the disturbing effect of CDF1 locus on NUE and NUE related traits was observed. Environment dependent and independent QTLs or MTAs were identified in our panel population, indicating the presence of genotype by environment interaction and effect of growing conditions on the expression of various sets of genes or QTLs.

We compared the QTL positions in the segregating backcross population (identified using linkage analysis) and the cultivar set (using genome wide association analysis, GWAS). Most QTLs did not co-localize between the two populations, however the SNP marker PotVar0026355 on chromosome V positioned at 4335324bp associated with NUE in the cultivar set, and the PotSNP573 marker positioned at 507660bp which was associated with NUE and the SSR marker Mando located at $4.67 \mathrm{Mb}$ which was associated with days to maturity in the backcross population are some of the QTLs which co-localized in the same genomic region on chromosome $\mathrm{V}$. The co-localization of these QTLs in both a biparental and an association mapping population validates the detected QTLs. AUC on chromosome III and TNPP and AP2 on chromosome VI co-located with QTLs for NUE. These two genomic regions may be useful for NUE improvement in potato other than chromosome $\mathrm{V}$, and these are independent of maturity type.

Trait-specific constitutive QTLs (QTLs for one specific trait observed in more than one environment) were detected for various NUE related traits in different environments. Constitutive QTLs for TYPP with peak markers solcap_snp_c2_26796 on chromosome IV 
and marker PotVar0060022 on chromosome XI were observed in two low $\mathrm{N}$ level environments under irrigation production system, indicating the QTLs may be low $\mathrm{N}$ as well as irrigation season dependent. This suggests the presence of QTL x environment interaction. However, the overall identified number of QTLs difference between low and high $\mathrm{N}$ level is lower than the QTL number difference between the two production seasons, suggesting QTL $\mathrm{x} N$ level interaction was lower than the QTL x production season interaction, in line with the stronger contribution of production system to the total genotype $\mathrm{x}$ environment interaction compared to $\mathrm{N}$ levels, as already discussed. To identify QTLs, various gene models were used in different association studies. In this study, we use additive, simplex and duplex dominance as well as general models, and most of the MTAs (including MTAs identified for NUE, AUC, TNPP, and TYPP) were detected using dominant genetic models. This indicates that the source of heritable variation for the identified MTAs is mostly due to dominant gene action or due to the interaction of alleles at a single locus, and that dominant gene effects are important in controlling potato NUE and NUE related traits. Overall our result demonstrated that the effect of production season was greater than the effect of $\mathrm{N}$ levels on NUE and NUE related traits under our experimental conditions.

The commercial potato cultivars evaluated in 2013 and 2015 were once again used for G x E interaction analysis. The study was conducted at three different locations in North-western Ethiopia: at Injibara and Debre-Tabor, under rain fed production conditions, and at Koga and Injibara in the dry season under irrigation at low and high $\mathrm{N}$ conditions. Each location combined with the production condition/season and N-levels was considered as a separate target environment making a total of eight test environments for this study. NUE was used as a target trait to evaluate the suitability of the test environments and the superiority of the cultivars in each environment in the $\mathrm{G} x \mathrm{E}$ interaction study. Considering $\mathrm{N}$ level as part of environment and NUE as a target trait helps for exploiting genetic and environmental resources efficiently and identify ideal test environments and superior genotypes for NUE improvement at low and high $\mathrm{N}$ levels in rain fed and irrigation production systems. Data were subjected to analysis of variance using Genstat 18.1 statistical software, and the $\mathrm{G} \times \mathrm{E}$ interaction was analysed using the genotype, and the genotype and environment (GGE) biplot model by GGE software.

The pooled analysis of variance over environments revealed that potato NUE is significantly $(\mathrm{P}<0.001)$ affected by the environment $(\mathrm{E})$, genotype $(\mathrm{G})$ and genotype-by-environment interaction (GE). The environment accounted for $79.6 \%$ of the total sum of square (SS) of (G 
$+\mathrm{E}+\mathrm{GE})$ variation which is the largest share of the variations. The genotype and genotype-byenvironment interaction respectively accounted for only $4.1 \%$ and $16.3 \%$ of the total sum of square variation. The significant effect of the $\mathrm{G} \times \mathrm{E}$ interaction in the combined analysis of variance suggested that the genotypes had variable performance in the tested environments.

In the GGE analysis, the percentage of GGE explained by PC1 and PC2 were $31.38 \%$ and $25.83 \%$ respectively, and the biplot explained $57.2 \%$ of the total SS variation relative to $\mathrm{G}$ and GE using environment standardized model. Although, multi-year data is required to use $\mathrm{G} \times \mathrm{E}$ interaction results, we found valuable information which suggests that an independent potato varietal selection program is required for each production system in north-western Ethiopia. The GGE analysis delimited the test environments into two mega-environments helpful in targeted evaluation of genotypes for NUE improvement. Regardless of the locations and $\mathrm{N}$ levels, the two mega-environments are rain fed mega-environment and irrigation megaenvironment. Testing environments were also identified within the mega-environment for proper selection of genotypes based on the basis of their representativeness and discriminating ability. Consequently, the high $\mathrm{N}$ level environments (E2 and E4) at both Debre-Tabor and Injibara were the most suitable environments in discriminating the potato cultivars and being representative test environments for NUE evaluation in rain fed mega-environments. Conversely, low $\mathrm{N}$ environment at Koga (E7) was the most suitable environment in discriminating the genotypes and as a representative of the irrigation mega-environment.

We identified three promising cultivars, Kuras, Asterix and Desiree in the basis of superior mean performance and stability across the test environments of rain fed mega-environment, and cultivars Hermes and Kuroda were identified as promising cultivars in the irrigation mega-environment. Characterization of potato germplasm for $\mathrm{N}$ use efficiency involves field evaluation for tuber yield and other NUE related traits under low and high $\mathrm{N}$ conditions. This thesis delivers an initial evidence of field screening potato for NUE improvement and identified important traits and their QTLs that help for the indirect selection in potato breeding for NUE. The QTLs identified in this thesis are potential interesting targets for potato breeding to improve NUE of the potato crop. Moreover, delineation of the test environments into two mega-environments proved to be helpful in targeted evaluation of genotypes for NUE improvement. 


\section{Acknowledgments}

It is my honour to acknowledge people those who helped and inspired me in the incredible journey of my PhD. First and foremost I would like to thank my promoter Prof. Rihcard G.F. Visser for giving me the opportunity to pursue my doctoral degree in the department of plant breeding under his promotion. Dear Richard, in spite of your busy schedule, I really appreciate your valuable support, comments and scientific guidance. Your comments and improvements on this thesis were always timely and helpful. I appreciate all your contributions of time and ideas to make my $\mathrm{PhD}$ experience productive and inspiring

I am very grateful to my co-promoter Dr.Gerard van der Linden for his perpetual scientific guidance, support and concern throughout my $\mathrm{PhD}$ period. Dear Gerard, you are my guide. During my arrival at wageningen you were with me to settle all the municipality and residence issues. From application of NUFFIC for fund to the end of my $\mathrm{PhD}$ you were helping me unconditionally. Your excellent advice and brotherhood approach have been invaluable both in academic and a personal level for which I am very grateful. Your encouragement to understand an idea in different angles helped me to explore my limitations which enhance my confidence to be an independent researcher. I am benefitted a lot from your genuine comments at every stage of my study. I really appreciate your way of supervision and strategic guidance throughout my $\mathrm{PhD}$ studies. Gerard, this thesis would not have been possible without your genuine support, patience and encouragement.

My special thanks goes to Dr. Marcos Malosetti for his insightful suggestions on the genotype $\mathrm{x}$ environment interaction part of my study. I extend my special gratefulness to Dr. Chris Maliepaard and Dr. Eric van de Weg for the discussion we had and valuable comments on QTL analysis and interpretation. I would like to thank, Prof. Anton Haverkort, Prof, Paul Struik, and Dr. Herman J. van Eck for the fruitful discussions we had on my research topic and other technical points during the early stage of this thesis work. I would like to thank all the members of the abiotic stress group for their support and valuable discussions at group meeting. Especially I would like to thank Ernest and Biructawit for their assistance during canopy cover data analysis and QTL analysis. Dear, Biructi and Ernest, I thank you very much, you were with me from data preparation to data analysis, and I learnt a lot from you guys. My special gratefulness goes to Mr. Dennis van Muijen for his help on the QTL analysis, and Mr. Molla Mekonen Kassie for his unreserved support during field experimentation. 
I am grateful to the Netherlands Universities Foundation For International Cooperation (NUFFIC) for funding the $\mathrm{PhD}$ program and the Amhara Region Agricultural Research Institute(ARARI) for granting me study leave. I thank especially the general director of ARARI, Dr.Birru Yitaferu, the former general director Dr. Fentahun Mengistu and former Deputy general director Dr. Yigzaw Desalegn for facilitating my study and field research work. My gratitude goes to HZPC Holland BV for the provision of planting materials with full shipping cost from the Netherlands to Ethiopia. I thank especially Dr. Maurice Schehr, Senior potato breeder in HZPC, for his facilitating role in the provision of planting materials. Dear Maurice, I really appreciate your interest to help me, without your participation and unconditional support this work would not have been reliable. I would like to thank our secretaries Letty, Janneke, and Nicole for their help with administrative issues, especially Janneke who helped me to settle problems related to my visa and flight processes, living allowance and all the bills after field works and conferences.

In my stay of plant breeding I had a very pleasant time with my officemates and I would like to express my gratitude to all of them: Dr. Daniel Danial, Madhuri, Abe, Manos, Michiel, and Karolina. Especially I am very grateful to my first officemates Daniel and Madhuri for helping me to adapt the new environment readily. I am thankful to the Ethiopian community, Sunday school group and friends at Wageningen for making my stay in the Netherlands pleasant: Mequanint, Yalemzewd, Setegn, Asmare, Mulate, Mezigebu, Belay Tseganeh, Dawit, Belay Tizazu, Yeshwas and Molla, thank you a lot for the times we spent together, and drinks and traditional meals we had together. I would like to thank the first people I met on my arrival to the Netherlands; Akalu, Moges and Drik Rolker who helped me to settle down in Wageningen. Dear Drik, I am grateful for your welcome and conveying me from schepol to Wageningen with your own car. Dear Akalu, you have a special place in my life. You are the one who encourage me to pursue my $\mathrm{PhD}$, and this endeavour would not have been completed without you. Thanks for being there for me for your everlasting advice, support, care and encouragement. I wish you a very happy life with your wonderful family.

I am grateful to the staff of Adet Agricultural Research Center for their support and encouragement during field experiment work. Especially I thank the potato research team, and people working at subcenters (Dr.Tesfaye Abebe, Alemu, Wubet, Balew, Zigale, Mamaru, Fentanesh, Zewudu, Gebru, Alebachew and Abebe) for their support during my field experiments. I would like to extend my gratitude to staff and fellow students in my institute 
ARARI for their assistance, discussion and friendship in my stay at Bahirdar for thesis write up: Mulugeta, Birhanu, Agegnehu, Mengistu, Menlik, Adebabay, Betew, Dejene and others. Especially I would like to thank Mulugeta for his help in the analysis and insight comment on the $\mathrm{G} \times \mathrm{E}$ interaction part of my study. Dear mule, I enjoyed a lot in all our stimulating scientific discussions and conversations. I also extend my warmest thanks to friends in Bahirdar: Dn.Dr. Tesfaye feyssa, Dr. Ermias, Dr. Semagn, Dr. Alemayehu, Dr. Minale, Ejgitu, Metalign and others who were in favour of my study.

My Sincere thanks goes to my family in Addis Ababa, Shiferaw and Amaledech for their unreserved support and hospitality in the course of my $\mathrm{PhD}$. I am very grateful to my mother Yeshalem Yematew (Tata) and my brother Tewodros Tesfaye for their encouragement, support and sense of responsibility to take care my wife and kids in my absence. I would like to express my deep gratefulness to my beloved wife Betelhem Mesfin, my daughter Hewan and my son Ananiya for their love, support and patience during my long stay away from home. Dear Betelhem (GZ), without your support and love my PhD study would have been very difficult. Dear my kids (Ema and Abu), you also contributed a lot for the success of this thesis. I used part of your time and your comfort to work on the thesis. You contributed even more; your sweet smile had a great power that always recharged me with new energy after a long exhausting day. Lastly but most importantly I want to thank my parents for their sacrifices and support throughout these years to accomplish my goals. I believe their blessings were with me in each step of success in my life.

Baye

September 2017, Wageningen, The Netherlands 


\section{About the author}

Baye Berihun Getahun was born $7^{\text {th }}$ December, 1971 in Gondar, Ethiopia. After completing his schooling, he joined for bachelors in Agriculture (1990-1994) at Alemaya University of Agriculture (AUA), Alemaya, Ethiopia. After completion of bachelors, he started his professional career as a crop production expert under the ministry of Agriculture in Amhara Region Bureau of Agriculture for 5 years. In 2001 he joined again the same University to pursue his masters in Agriculture (2001-2003) with specialization in horticulture. As part of his MSc, he worked on the thesis entitled 'Variability and association of tuber yield and related traits in potato' in the department of plant sciences. After completion of MSc in June 2003 to date, he worked as a researcher in Amhara Region Agricultural Research Institute, (ARARI) based at Adet Agricultural Research Center. In his stay at Adet Agricultural Research Center, from April, 2008 to January, 2011 he served as crop Research directorate director, and from June 2011 to June 2012 as National Potato Research Program Coordinator. In June, 2012 he started his $\mathrm{PhD}$ in Plant breeding at Wageningen University and Research, the Netherlands. 


\section{List of Publications}

Baye . B.Getahun Ravishankar.H and Ha.Singh (2005) Variability and Association of Tuber yield and related traits. Ethiopian Journal of Agricultural Sciences (EJAS).Vol: 18 No.1

Baye B. Getahun (2011) Effect of mulching and amount of water on the yield of tomato under drip irrigation Jour of Hort and Forestry Vol. 3(7), pp. 200-206,

Baye Berihun Getahun, Molla Mekonen Kassie, Richard RGF Visser, Gerard C van der Linden(2017) Genetic diversity of potato cultivars for nitrogen use efficiency under contrasting nitrogen regimes. (Submitted)

Baye Berihun Getahun, Richard GF Visser, C. Gerard van der Linden (2017) Identification of QTLs associated with nitrogen use efficiency and related traits in a diploid backcross potato population (Submitted)

\section{Conference abstracts and papers}

Baye Berihun Getahun, Molla Mekonen Kassie, RGF Richard Visser, C Gerard van der Linden(2016) Assessment of genetic diversity and relationships for agronomic nitrogen use efficiency and related traits in potato (Solanum tuberosum L.) under contrasting $\mathrm{N}$ regimes, APA, $10^{\text {th }}$ triennial conference, Oct, 9-13, Addsi Ababa, Ethiopia

Baye Berihun Getahun and Gebremedhin Woldegiorgis (2013) Potato research and development in Ethiopia, Proceeding of the national Workshop on seed potato tuber production and dissemination, 12-14 march 2012, bahirdar , Ethiopia

Yalefal T, Tesfaye A, Daniel T, Shimeles A, Baye BG, Gedif M, and Alemu W (2007) Potato technology transfer experiences and future recommendation for sustainable development of the commodity in parts of Western Amhara Region. Proceedings of the $1^{\text {st }}$ Amhara Region Regional workshop on potato Research and development. Achievments, Transfer of experience and future directions Dec, 20- 21, 2007

Tesfaye A, Baye BG, Yazie C, Gedif M , and Alemu W (2008) Experience challenge and future recommendation to create sustainable community based informal potato seed scheme in parts of west Amhara . Proceedings of the Ethiopian Horticultural Science Society. Improving productivity of horticultural crops for sustainable Economic Growth January15-16, 2009. 
Baye B. Getahun and Mehatemwork.M.2007.Evaluation of different rate of Fertilizer Recommendations for Potato in Major growing Areas of East Gojam. Proceedings of the $3^{\text {rd }}$ annual conference on completed crop research activities August 2009. Amhara Regional Agricultural Research Institute (ARARI). Bahirdar, Ethiopia.

Baye.B Getahun and Mehatemwork.M (2007) Effect of mulching and amount of water on the yield of tomato under Drip Irrigation. Proceedings of the $3^{\text {rd }}$ annual conference on completed crop research activities August 2009. Amhara Regional Agricultural Research Institute (ARARI). Bahirdar, Ethiopia

Baye B .Getahun (2007) Effect of Irrigation frequency and N-fertilizer on the tuber yield of Potato (Solanum Tuberosum L) Proceedings of the $1^{\text {st }}$ National workshop on potato Research for development and poverty reduction March, 11- 13, 2008. Ethiopian Institute of Agricultural Research (EIAR). Holetta, Ethiopia 


\section{Education Certificate}

\section{Educational statement of the Graduate School of Experimental Plant Sciences}

Issued to: Baye Berihun Getahun

Date: 18 September 2017

Group: Plant Breeding

University: Wageningen University \& Research

\section{1) Start-up phase \\ - First presentation of your project}

Genetic diversity of potato for nitrogen use efficiency under low input conditions in Ethiopia

- Writing or rewriting a project proposal

The genetic basis of nitrogen use efficiency

Writing a review or book chapter

- MSc courses

Genomics

Laboratory use of Isotopes

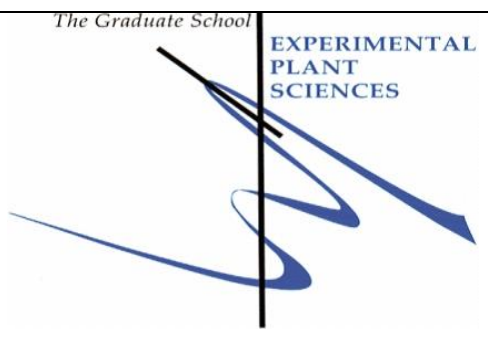

date

Dec 18, 2012

Jul 1- 30 Aug, 2012

Sep 1-30 Oct, 2012

12.5 credit points

\section{2) Scientific Exposure}

Subtotal Start- Phase

\section{- EPS PhD student days}

EPS PhD student days, University of Amsterdam

EPS PhD student days, Soest, StayOkay

\section{- EPS theme symposia}

EPS theme 4 symposium Genome biology, University of

Nijmegen

EPS theme 2 symposium Interaction between plants and biotic agents and willie Commelin day Utrecht University

- EPS Lunteren days and other National Platforms

NOW-ALW meeting experimental plant sciences; Lunteren, NL

- Seminars (series), workshops and symposia

EPS seminar, use of resurrection as models to understand how plants tolerate extreme water loss: A system biology approach with applications for making drought tolerant crop, Wageningen EPS seminar, Strong selection on the genes controlling complex traits in complex environment, Wageningen

EPS seminar, Ecological principle of plants, plant interaction

(linking crops and natural system), Wageningen

EPS seminar, Linkage analysis and QTL mapping in autotetrploid potato using SNP dosage data

Plant Breeding research day, Wageningen University

\section{$\checkmark$ Seminar Plus}

- International symposia and congresses

Conference next generation Plant Breeding, Wageningen

University

\section{- Presentations}

European Potato Research Association, Italy (Oral)

African Potato Association, Ethiopia (Oral)

\section{$\underline{\text { Date }}$}

Dec 12, 2012

Jan 28-29, 2016

Dec 12, 2012

Jan 24, 2013

Apr 11-12, 2016

Jun 26, 2012

Dec 10, 2012

Dec 11, 2012

Sep 7, 2015

Sep 29, 2015

Nov 11-14, 2012

Nov 14-17, 2015

Oct 9-13, 2016

Dec, 2012 \& Jun, 2014 
IAB interview

Excursions

Presentation and Excursions to HZPC Potato breeding Company

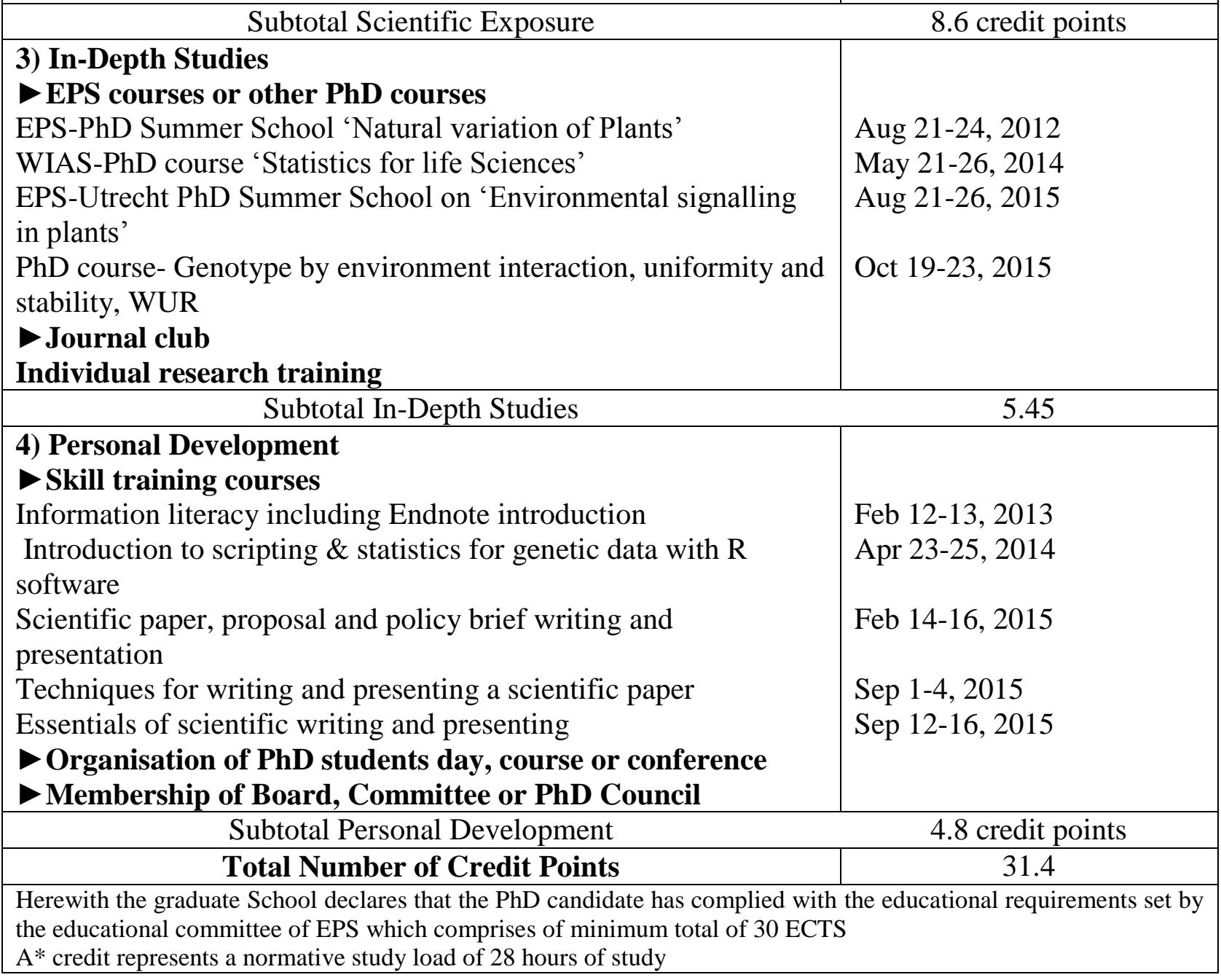




\section{Funding}

Funding for this study was provided by the Netherlands Universities Foundation For International Cooperation (NUFFIC): an NFP fellowship under award number NFPPhD.11/804

Cover design by Baye Berihun Getahun

Printed by GVO Drukkers \& Vormgevers B.V. Ponson \& Looijen, Ede, The Netherlands. 